\title{
Guiding Light
}

The Architecture of Sleep for Dementia

Scott Kluger

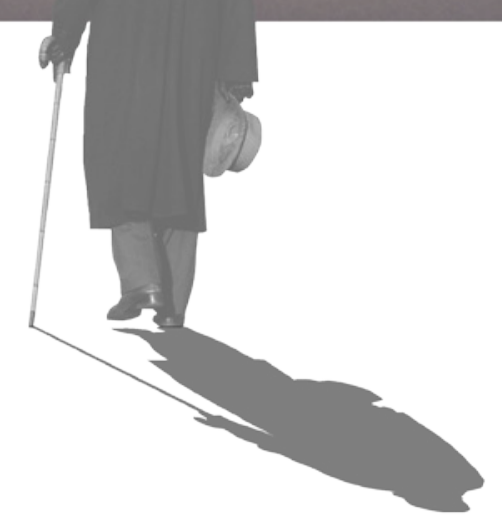





\title{
Guiding Light
}

The Architecture of Sleep for Dementia

\author{
By Scott Kluger
}

A 120 point thesis

submitted to the Victoria University of Wellington

in partial fulfilment of the requirements for the degree of

Master of Architecture (Professional)

Victoria University of Wellington

School of Architecture

2016 


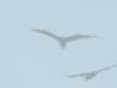

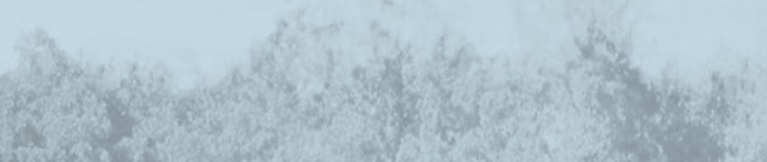

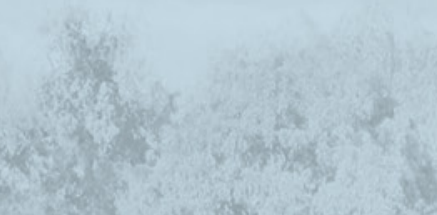
25.

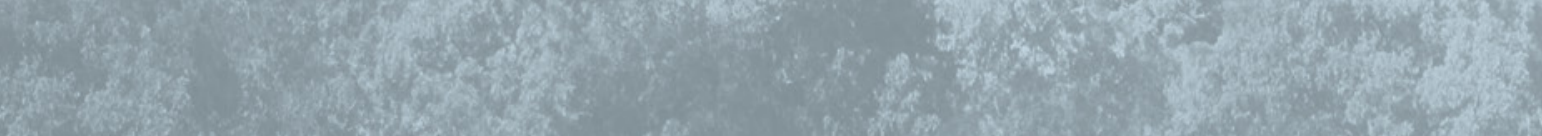
$\lim ^{2}-x^{2}=x^{2}$

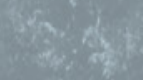
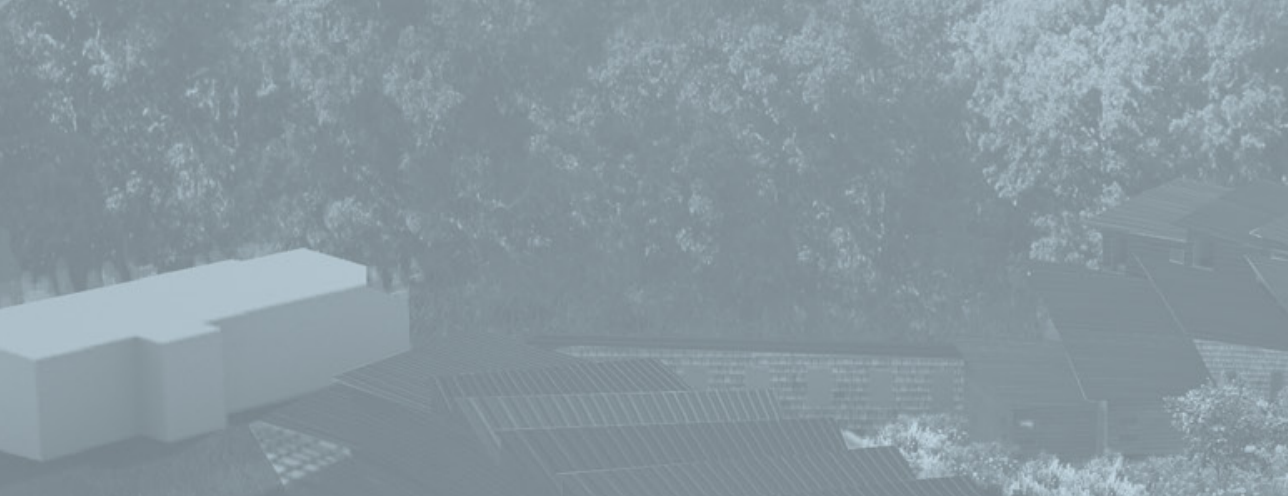

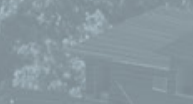

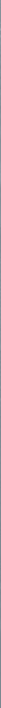




\section{Acknowledgments}

I would like to thank my supervisor Jacqueline McIntosh; your constant enthusiasm and guidance throughout the year has been invaluable.

To Mum and Dad, thanks for your unwavering support and encouragement throughout my studies.

And finally, thanks to all my classmates and friends in Wellington who have made this journey in the last five years truly memorable. 


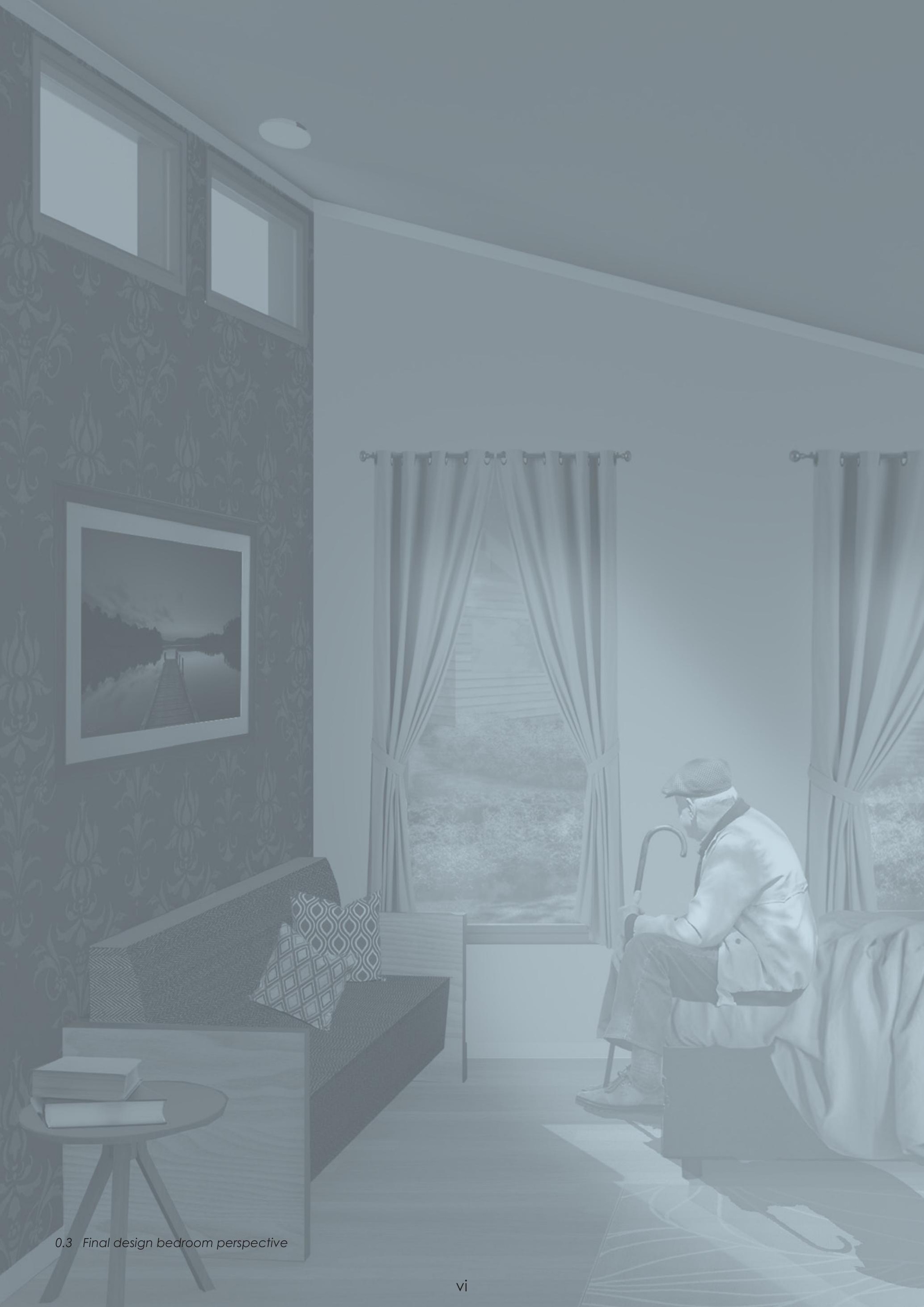




\section{Abstract}

Disruptive sleeping behaviours are a key symptom of dementia that accelerates transition from the home towards full time institutional care. For thousands of families in New Zealand, respite facilities offer a temporary relief from this symptom in an attempt to prolong care at home. However the predominant use of pharmaceutical therapy coupled with unstimulating care environments leads to sleeping behaviours deteriorating upon returning home.

In order to provide an alternative approach to pharmaceutical therapy and research towards treating disruptive sleep behaviour, this thesis addresses pre-existing inter-disciplinary literature, field trips, international precedents and an iterative method of design to investigate: How can the architecture of a respite facility improve sleeping behaviours and instigate meaningful environmental research within dementia care?

Environmental strategies involving light, movement and community were identified as key objectives towards improving sleep behaviour within the design. Introducing a shared courtyard with the public that facilitated continuous movement alongside the circadian rhythms of the sun, allowed a design that would engage with all three objectives in order to improve sleep behaviour of residents with mild symptoms of dementia. As symptoms progress, the adoption of an artificial lighting environment in a purpose built sleep lab allowed a space for scientific enquiry to the nature and treatment of sleep for those with later stages of dementia. The final design integrates both natural and artificial environments into a single respite facility, strengthening its therapeutic potential to prolong home care for the thousands of families affected by dementia in New Zealand. 


\section{Table of Contents}

Acknowledgements

Abstract vii

Introduction

Literature Review

Light as therapy

Movement as therapy

20

Community as therapy

Project Review

De Hogeweyk

The Five Gardens

Alzheimer's Respite Centre

Village at the Park

Te Hopai

Sprott House

Massey University Sleep/Wake Centre

Designing Sleep Therapy For Dementia 


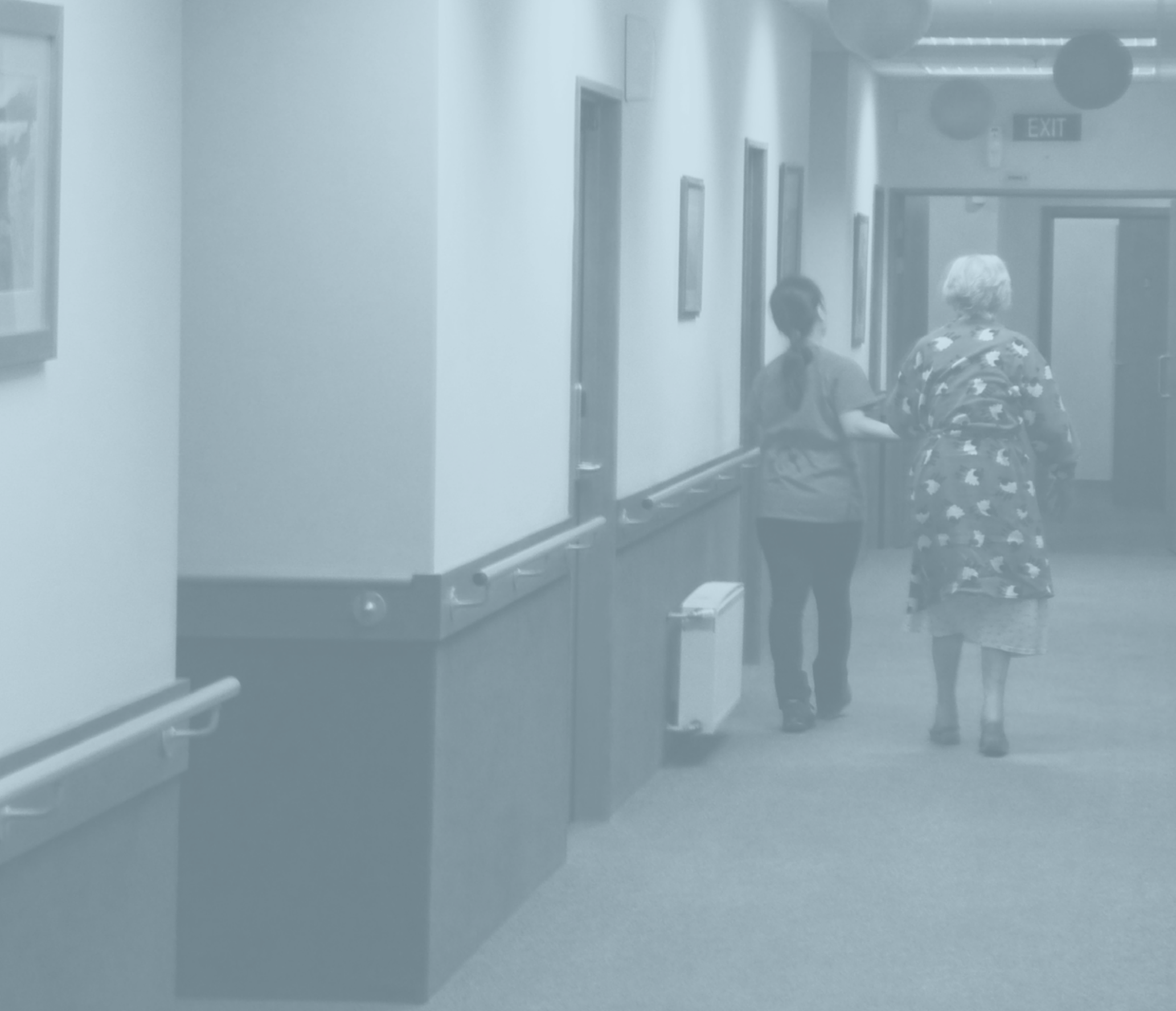


1

Introduction 
Watching the gradual deterioration of a family member affected by dementia has become an emotional reality for thousands of families providing care throughout New Zealand. Unaccustomed with the knowledge and skills to manage the behaviour and symptoms of dementia, family carers face an ongoing struggle to maintain quality care at home. Memory loss, incontinence, disruptive sleep, wandering and aggressive behaviour are common symptoms that fall under the umbrella term of dementia (Figure 1.2), a group of brain diseases that leads to a deterioration of the brain and affects cognitive abilities (Figure 1.3).

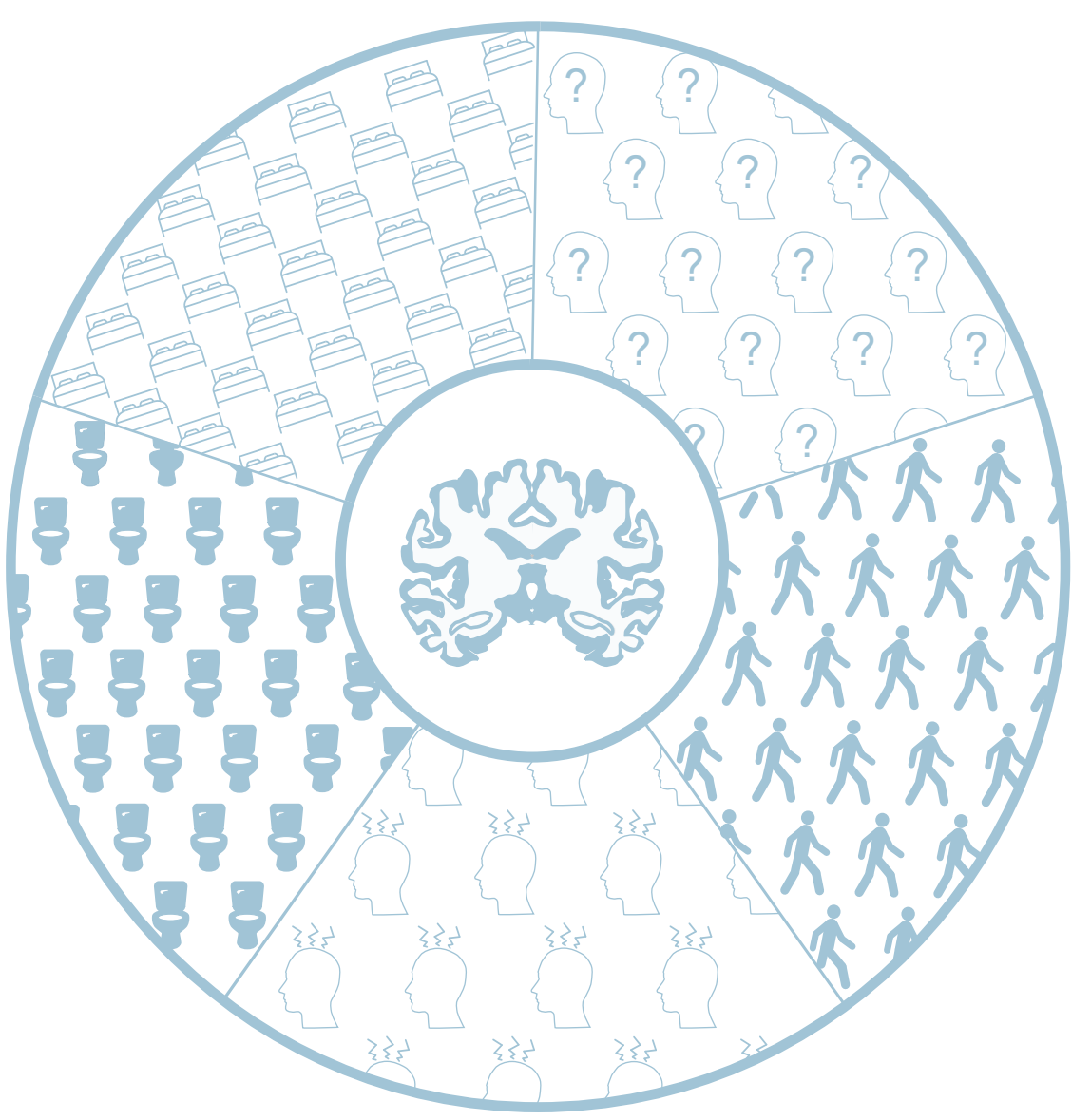

1.2 Symptoms of dementia 


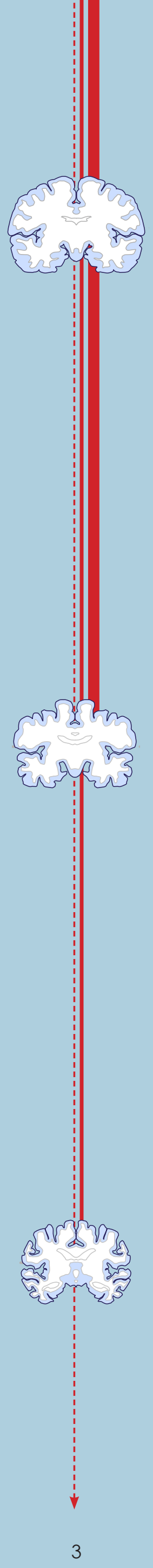

1.3 Dementia progression 


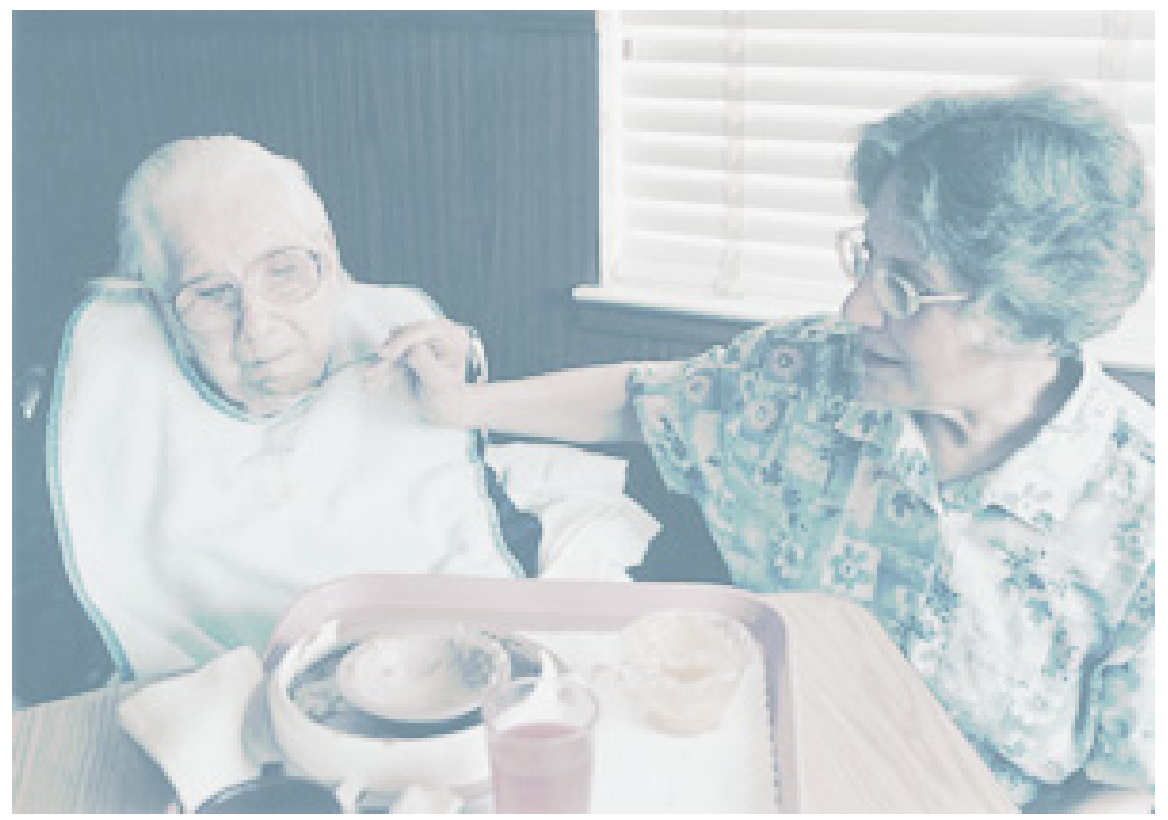

1.4 Dementia home care

Progressive symptoms of dementia increase dependency on family carers creating physical, psychological, emotional, social and financial burdens (Figure 1.4), generating a multidimensional response known as caregiver burden (Werner 89) (Figure 1.6). New research has highlighted that two out of every three New Zealanders know, or have known, someone affected by dementia, with $64 \%$ of these people being family members (Alzheimers New Zealand 6). A significant proportion (1/3) of those family members are directly involved in their care (lbid), emphasizing that dementia touches "the vast majority of New Zealanders, and for many of us it directly impacts our family and our daily lives"(Ibid). Current reports estimate the growth of dementia to triple by 2050 (Mathewson) (Figure 1.5). This increase in prevalence not only affects those with dementia, but also the health and well-being for countless families who provide care throughout New Zealand.

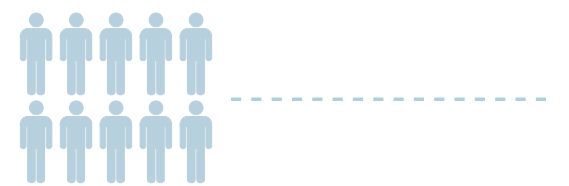

2014

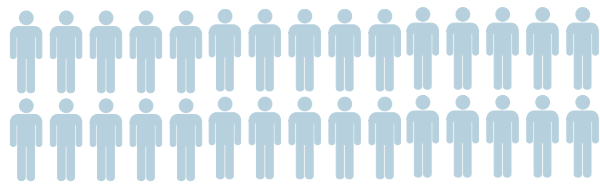

2050

$=5,000$ people

1.5 Estimated increase of dementia in New Zealand 


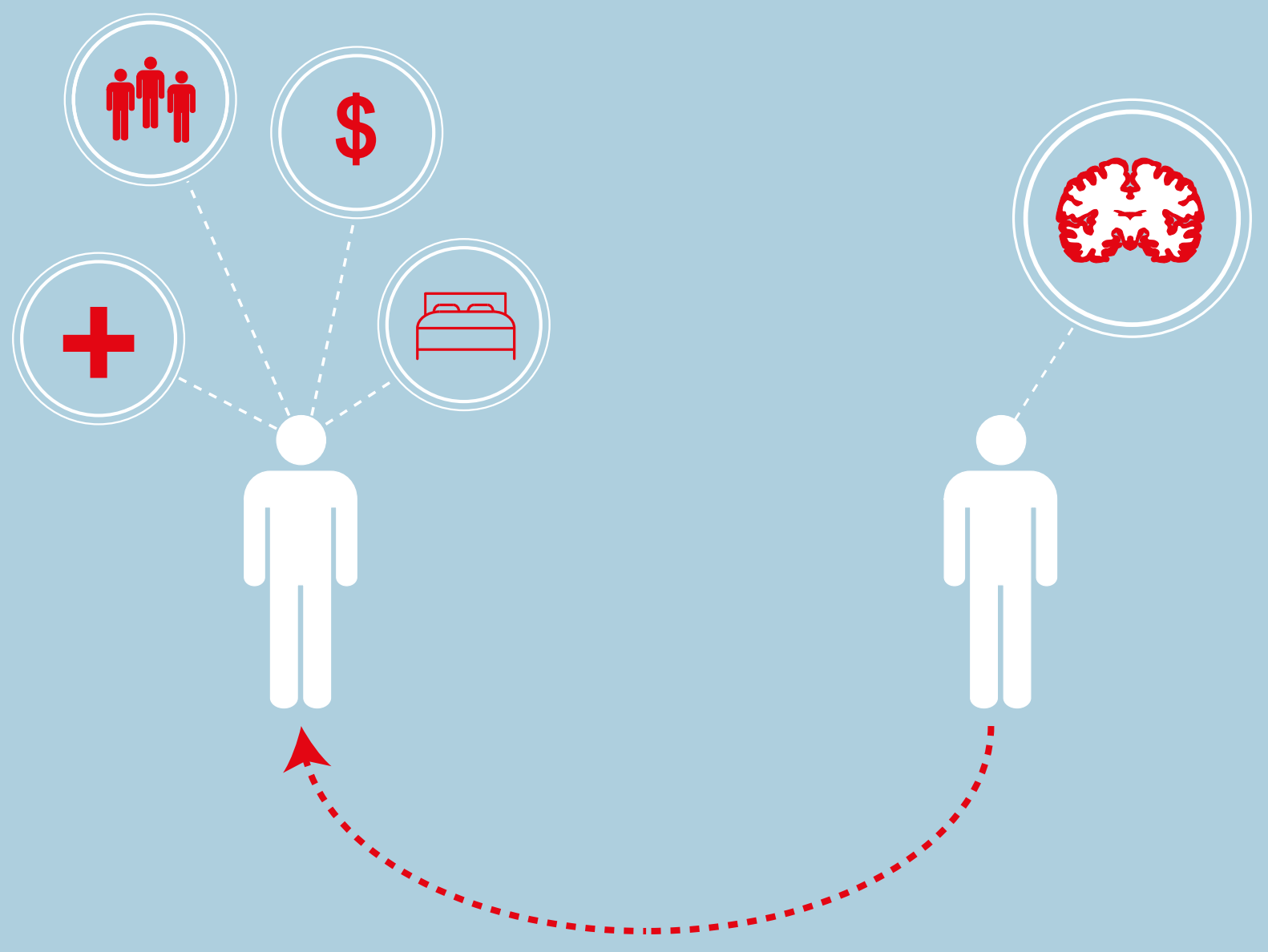

1.6 Family caregiver burden 
The current model of dementia care promoted by the Ministry of Health emphasizes home care and independent living rather than institutional care. Inherent in that model is the need for respite facilities to provide short-term relief for family carers. People with dementia are affected by disrupted sleep, as they "take longer to get to sleep, stay awake longer when disturbed, tend to be more active during periods of wakefulness, and are more likely to sleep during the day "(Lee 252). Sleep deprivation of the family caregiver is also a major contributor towards using respite facilities, as "reductions in caregiver sleep quality and stamina are among the most frequently cited reasons for the breakdown of caregiving at home" (Ibid). Family caregivers often cite "sleep disturbances, including night wandering and confusion, as the main reason for institutionalizing the elderly" (Klaffke 168) (Figure 1.7).

During periods of respite, improvement of family caregiver sleep behaviour is evident. However, "for patients, respite care worsened already disturbed sleep patterns, demonstrating the need to target sleep management of patients during respite care" (Boltz 219). The transition from the home environment to an institutional environment during respite periods presents a conflicting dichotomy between improved family caregiver wellbeing at the expense of the wellbeing of those affected by dementia (Figure 1.8). The need to adapt the respite environment to improve sleeping behaviours for those affected by dementia is necessary if institutional environments are to benefit both the family caregiver and those with dementia.

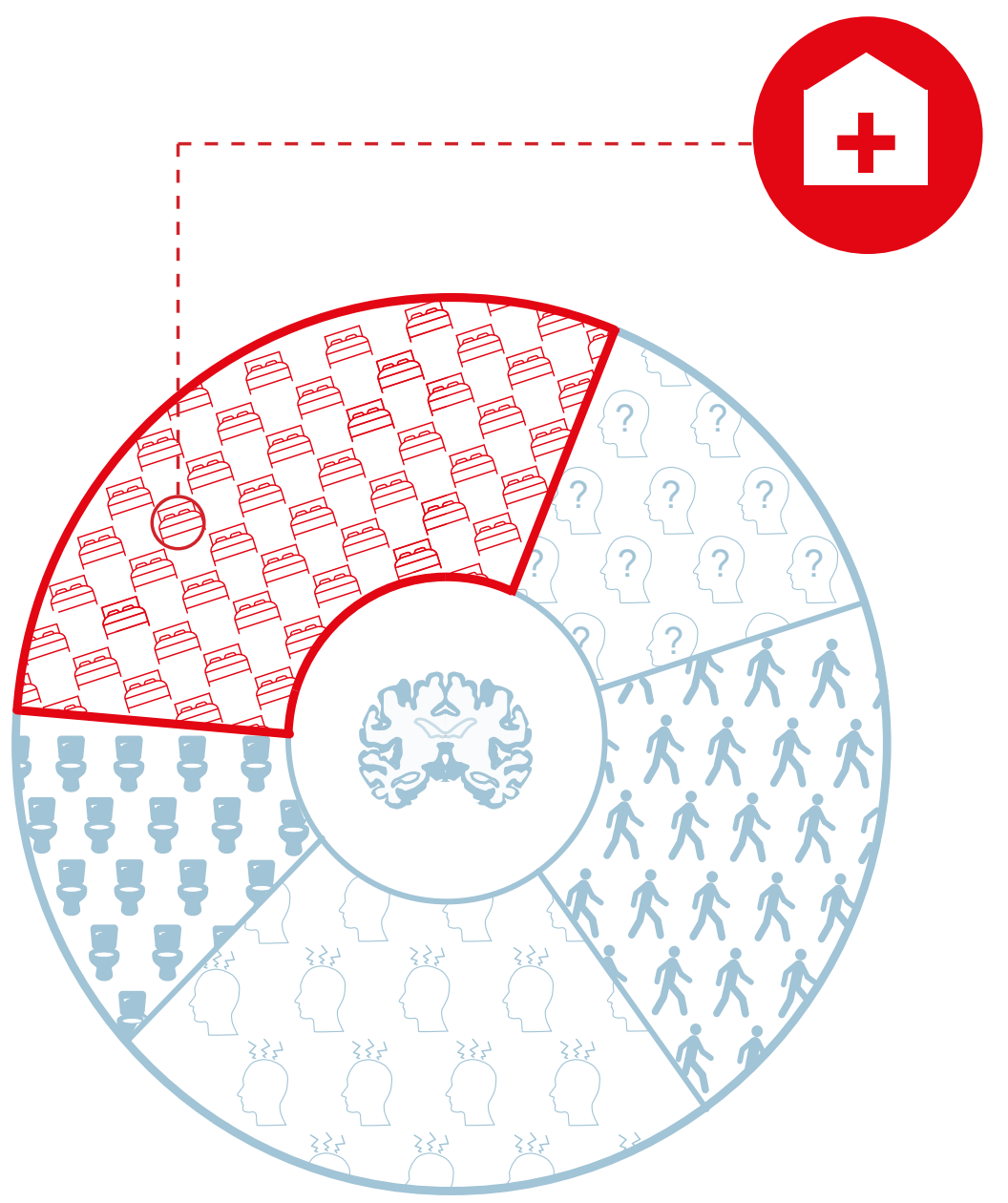

1.7 Key symptom leading to institutional care 
Family Caregiver

Percentage of days with assumed sleep

$\%$

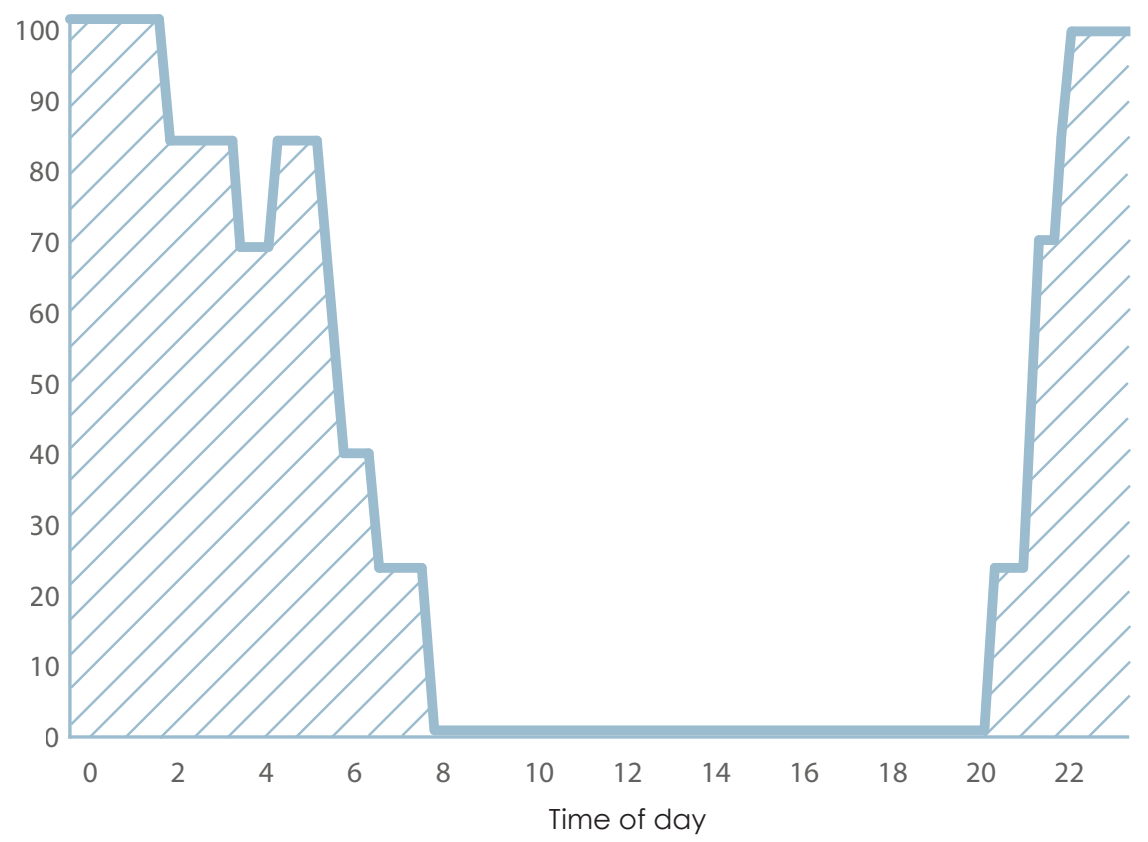

Person affected by dementia

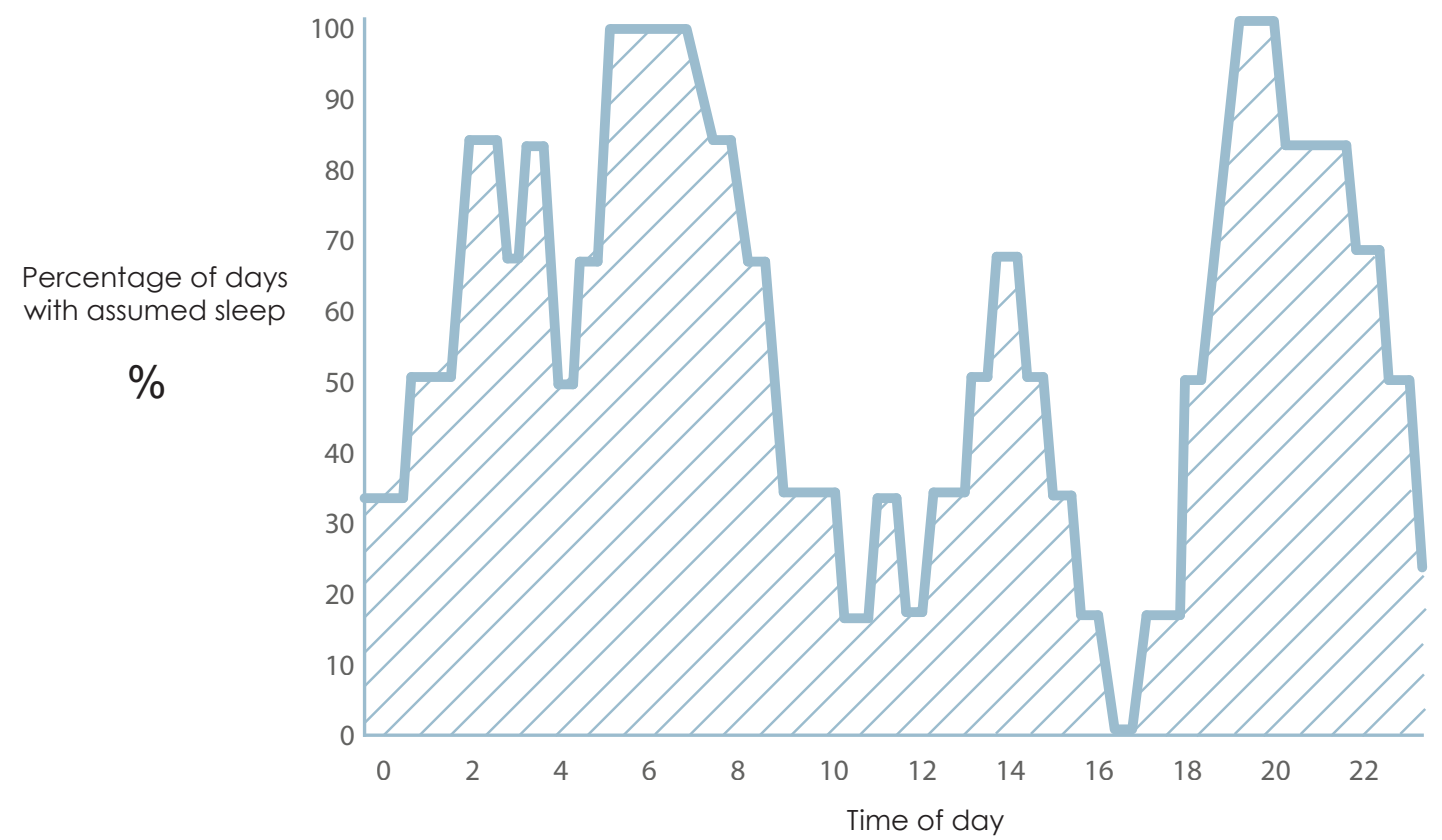

1.8 Sleep behaviour of family caregiver vs person affected by dementia (Gibson 43) 
Respite facilities are medically orientated, as people are admitted to "institutional settings where basic and medical needs are met but homelike attributes are often lacking. Environments can be stark and meaningful activity limited" (Reimer 1085). The lack of stimulating and meaningful activity within institutional environments results in a dependency on pharmaceutical interventions for therapy, reflecting the traditional view that the "physical environment is not considered an active component of treatment" (Clakins 145). Although pharmaceutical interventions provide improvements towards dementia related symptoms, the "side effects of medications can exasperate cognitive difficulties and increase the risk of adverse outcomes" (Bartle). Commonly used treatments (such as Donepezil) can stimulate the nervous system and increase the risk of disturbed dreams (Ibid). The limitations of pharmaceutical treatment coupled with an inappropriately designed age care environment were identified as contributing factors towards disturbed patient sleep following respite (Lee 257). There is a need for these facilities to "focus on strategies that optimize the sleep quality of patients in respite care and broaden and maintain these demonstrated benefits to spousal and non spousal caregivers" (Ibid 257). However, research and funding towards dementia treatment reflects a continuing preference towards pharmaceutical intervention rather than environmental, continuing trends that have devalued the design of dementia care environments (Figure 1.9).

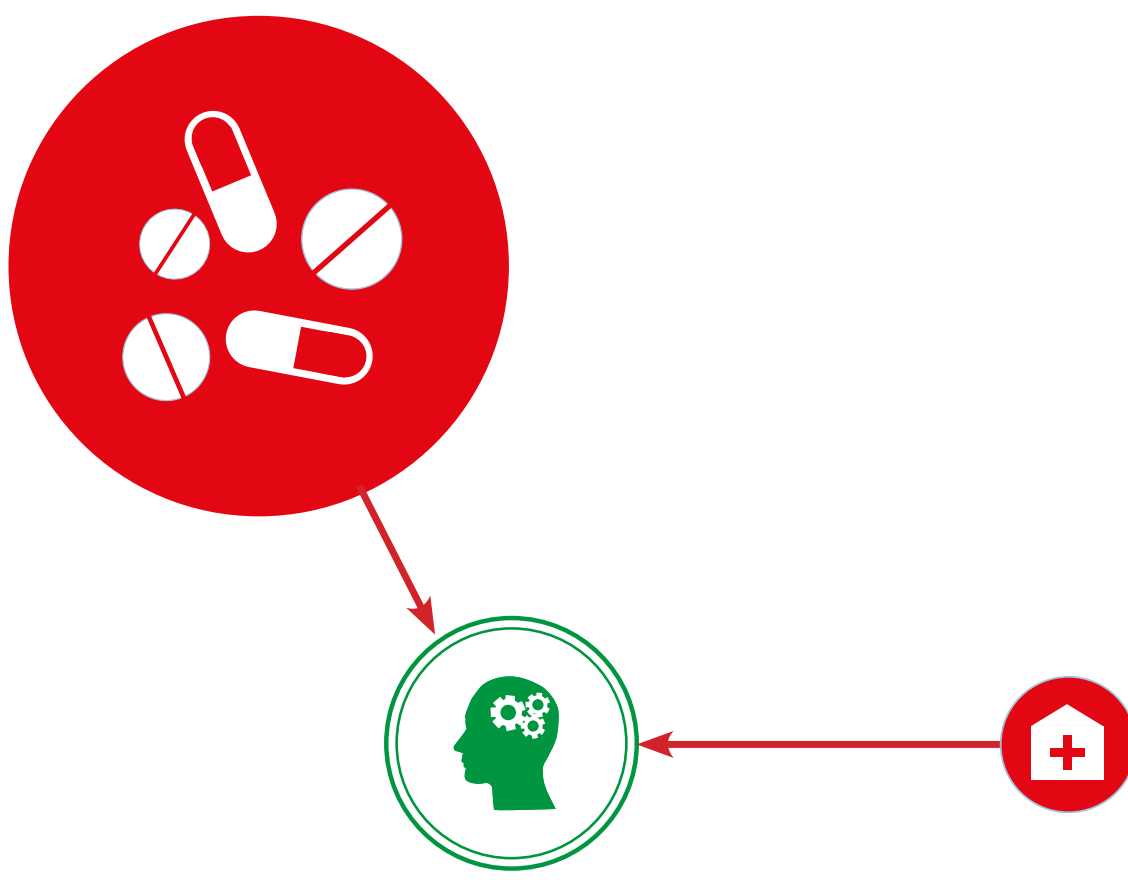

1.9 Influence of environmental therapy for dementia care 


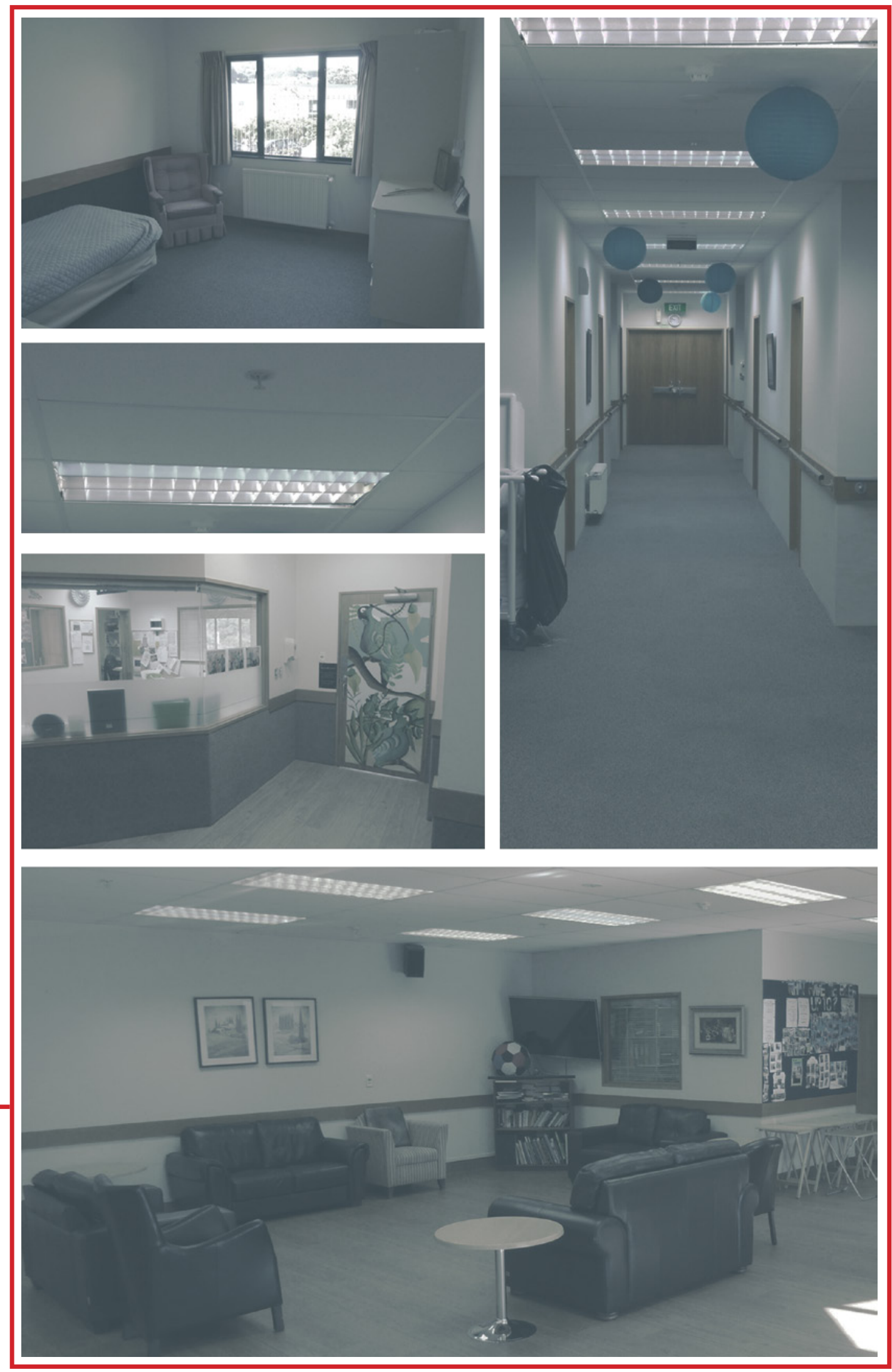


New Zealand's is one of the lowest contributors to dementia research out of all OECD countries (The New Zealand Herald), with the first dementia research clinic only opening in 2014 (University of Auckland). The continuing emphasis on pharmaceutical intervention, testing novel drugs for patients to "participate in a range of preliminary clinical trials, developed as part of the research programme" (The University of Auckland), maintains this emphasis of pharmaceutical intervention for therapy. Ministry of Health geriatrician Dr Phil Wood from the research clinic identified that there had not been any new method for treating dementia in 20 years, and that he was disappointed with the process of medication, focusing more on the relationship between lifestyle and dementia (Radio New Zealand). However, the ability to test these environmental interventions within the research clinic are limited by its pre-exiting institutional setting, the ad hoc addition of the clinic reducing the impact of environmental interventions by the institutional aesthetic within Auckland hospital.

While there is a growing need for respite facilities to provide temporary relief for family caregivers, the negative impact these facilities contribute towards disrupted sleep behaviour for residents only increases their burden of care. Combined with New Zealand's response towards dementia research illustrating further disparity between the environment and dementia therapy, a new direction is required if the environment is to be considered an active component of therapy (Figure 1.10). This thesis seeks to address this missed opportunity by questioning: How can the architecture of a respite facility improve sleeping behaviours and instigate meaningful environmental research within dementia care? 


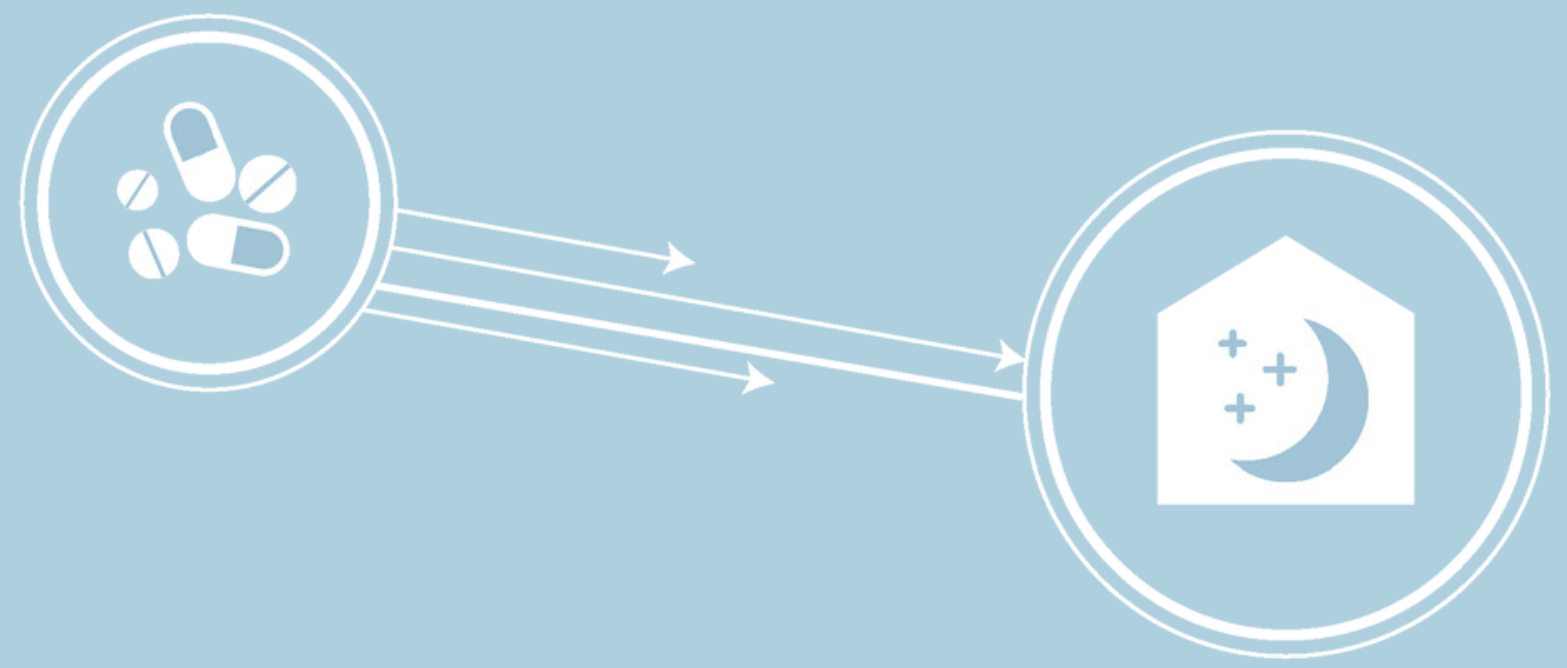

1.10 Shift towards architecture for sleep therapy 


\section{Research Methodology}

This thesis structure follows a design through research approach that embeds medical theory and practice towards a design led process, strengthening the connection between the two disciplines (Figure 1.11). An interdisciplinary literature review outlined the problems and design objectives towards improving sleep behaviour. From these objectives design criteria were formed to analyse case studies and to test the objectives within international and local contexts, the latter necessitating field trips and analysis to enhance the quality of observation and assessment of the designs. Implementing the design criteria in conjunction with an iterative design process ensured a scientific method of evaluating experiments, concepts and ideas alongside the desired design objectives. A range of both analogue and digital design methods were implemented in the process, followed by multiple explorations, reviews and reflections that were guided within the parameters of the design criteria. Critiques by architectural academics, medical practitioners, caregivers, practicing architects and peers allowed refinement to the evaluation of designs within the process.

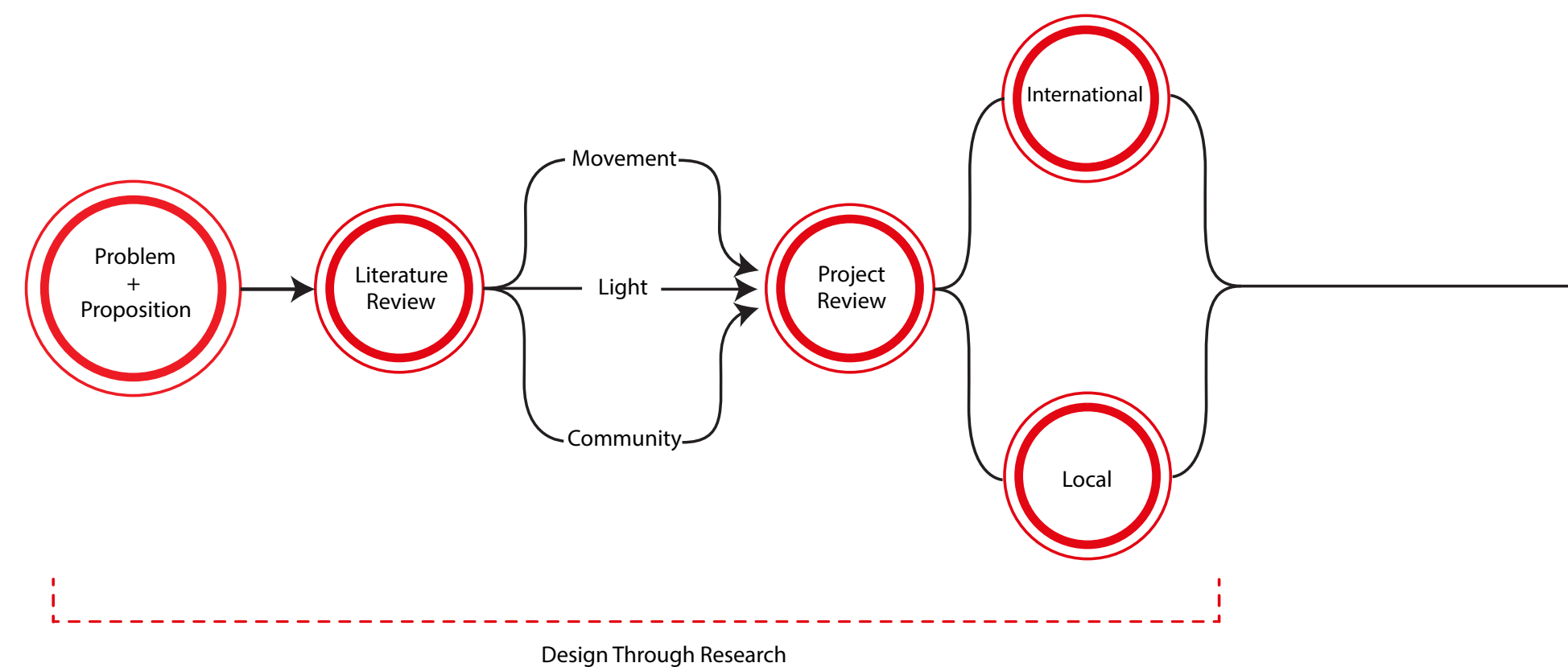

1.11 Thesis structure diagram 


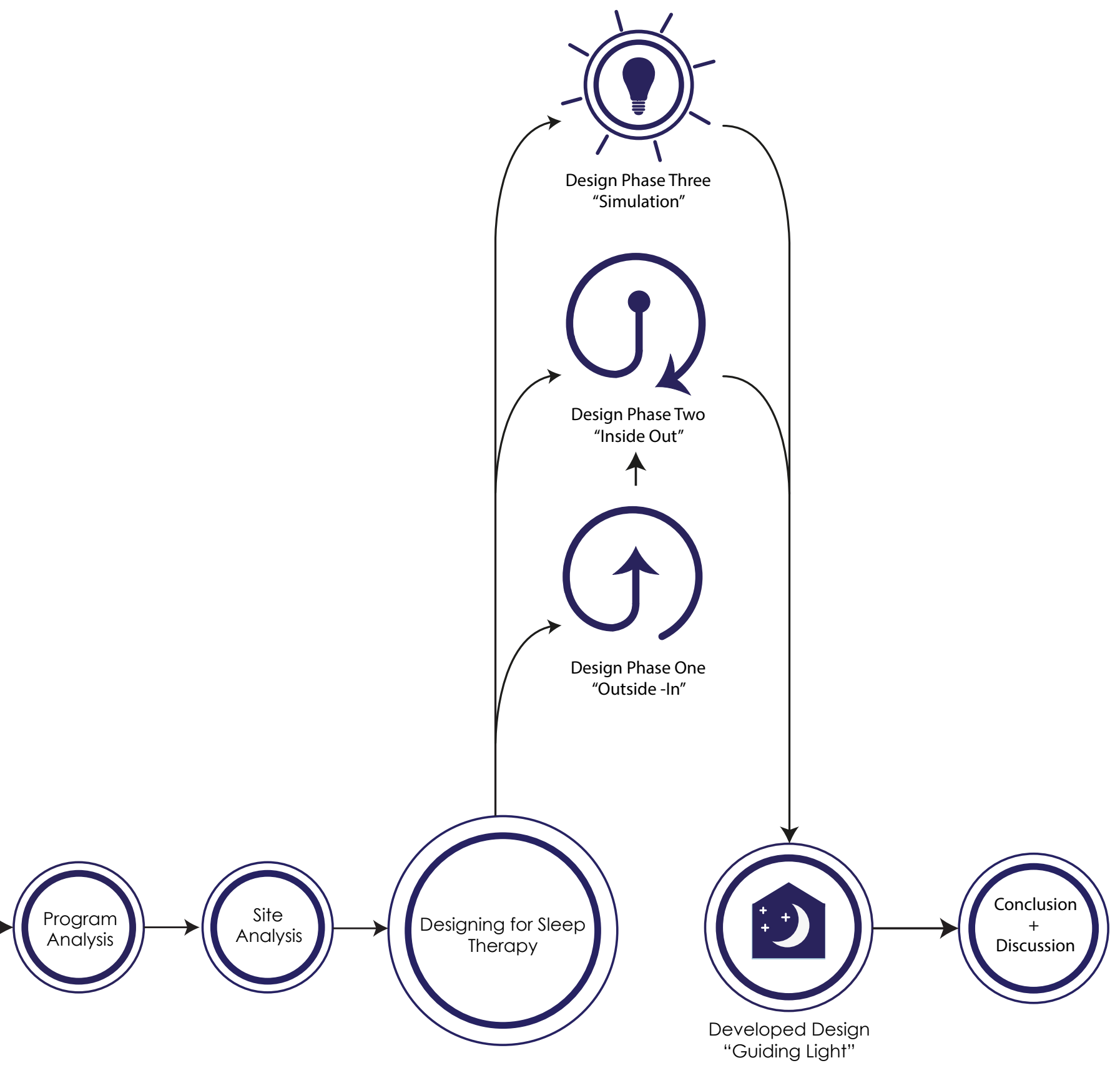

Research Through Design 


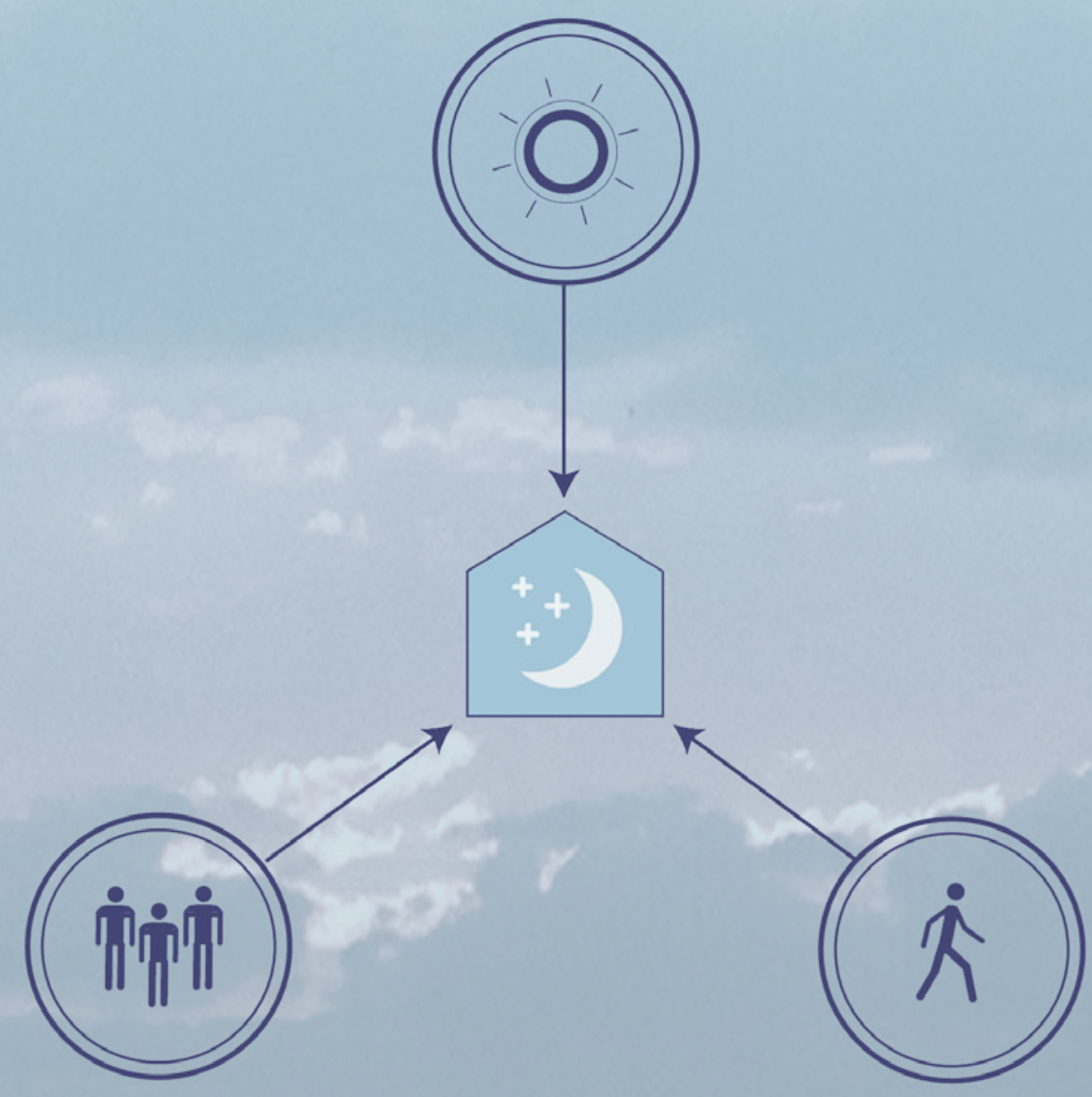




\section{Literature Review}

The role of architecture within dementia research has grown, highlighting a shift from a predominant medical model of care towards a more interdisciplinary approach towards therapy. The following literature analysis seeks to strengthen this shift by integrating the two disciplines together, allowing the combination of medical evidence alongside architectural theory to improve the therapeutic potential of the built environment. Interventions such as light, movement and community have been identified as key environmental strategies towards improving the sleeping behaviour and wellbeing for those affected by dementia (Figure 2.1). 


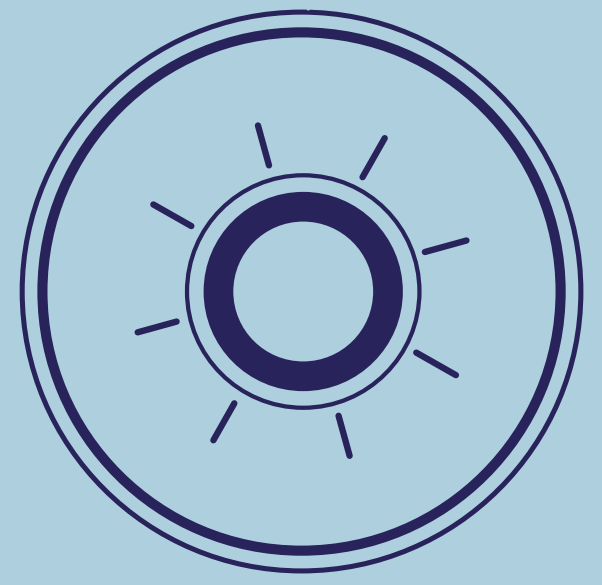

Light as therapy 
Light as a therapeutic intervention for dementia care has been shown to reduce problematic symptoms such as disrupted sleep cycles and night time wandering. Specifying light exposure at different times of the day can help to regulate the body's natural rhythms, as the "suprachiasmatic nucleus of the brain serves as an hourglass timer or pacemaker for our internal clock or circadian rhythm and is directly influenced by light intensity" (Boubekri 54). Increasing light intensity earlier in the day to provide active stimulation and reduce drowsiness has been proven successful, "as light therapy is most effective in treating circadian misalignment if administered shortly after awakening" (Gooley 670) (Figure 2.2). In contrast, minimizing light in the afternoon and evening can help reduce "sundowning symptoms" that cause increased agitation and confusion, as "exposure to light at night can disturb sleep and suppress melatonin production" (Feddersen 107). By introducing cyclical patterns of light as a design tool for improving circadian rhythms, a systematic exposure of light can help to reduce the severity of disrupted sleep symptoms (Figure 2.3).

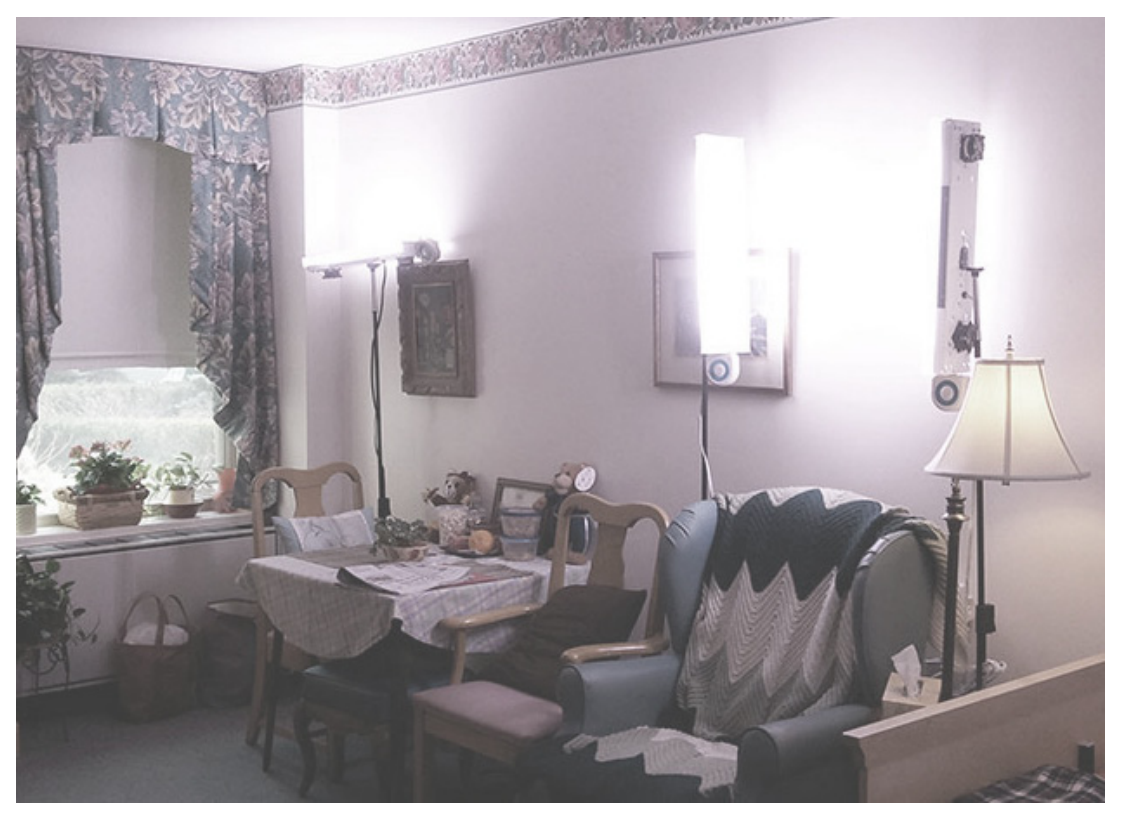

2.2 Lighting system to provide high circadian stimulation 


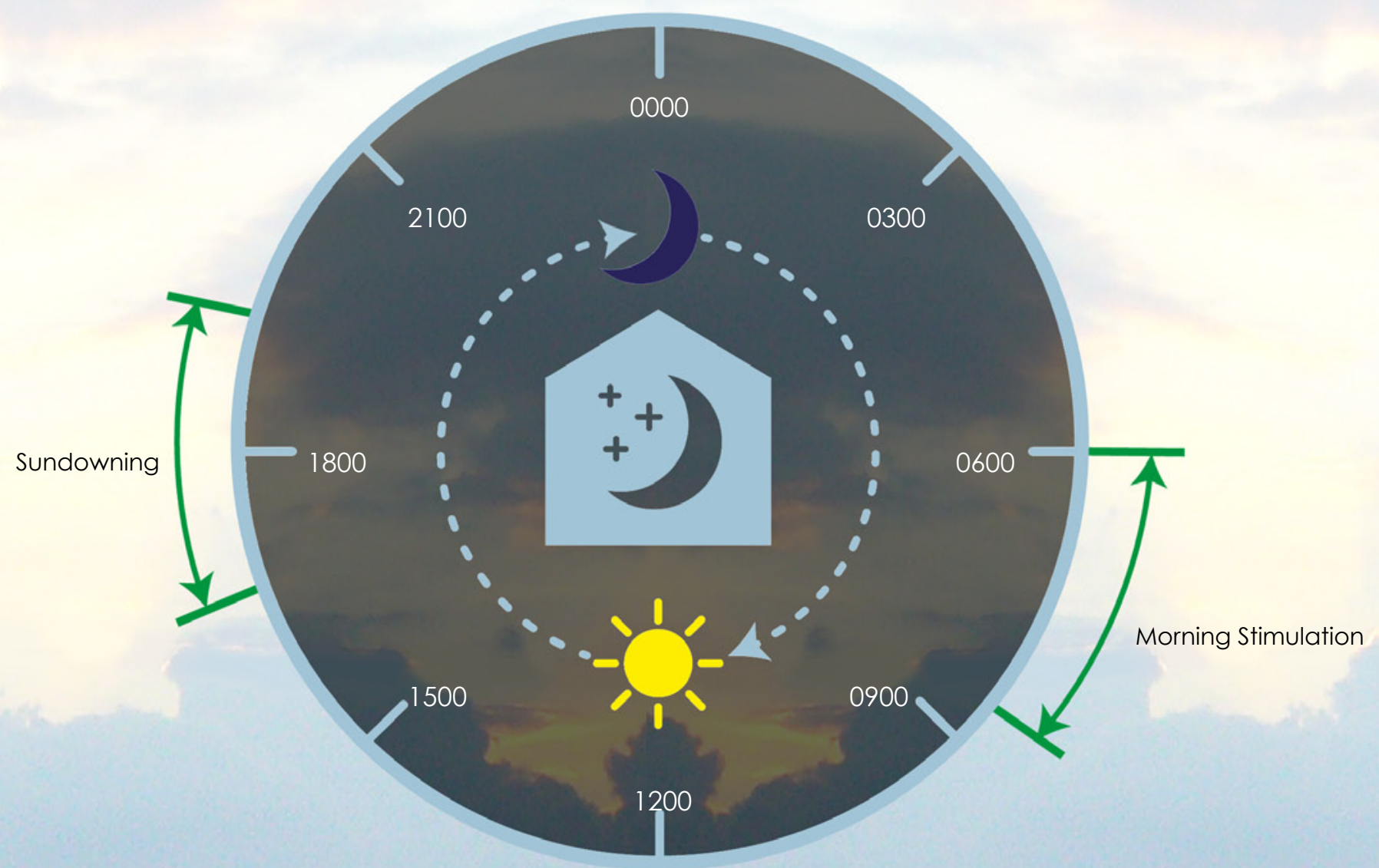


The use of light therapy to improve circadian rhythms highlights a potential shift from a medical model of care to an architectural interstitium, a notion coined by architectural theorist Ralph Knowles. Knowles identifies the interstitium as a building that responds to changing light qualities, emphasizing seasonal variation and sun orientation as key drivers towards design (Knowles 155). This highlights a dynamic response towards natural light patterns, where "flexible structures, comprising either the whole of some part of a building, might expand and contract, making use of different seasonal envelopes" (Ibid 155). Furthermore, Knowles recognizes the positive influence natural light can offer towards creating familial atmospheres, "as passing sensations rhythmically alter the proportions of space" (Ibid 9), identifying that "just as in nature, rhythmic boundaries also define the spaces we ourselves make and occupy" (Ibid 9). Emphasizing these daily patterns of natural light could help to create familiar atmospheres for residents within a seemingly unfamiliar environment.

The role of natural light therapy is also evident in utilizing outdoor spaces for dementia residents. The garden has been recognized as a therapeutic tool for cognitive stimulation as the "benefits of the garden were thought to occur through two mechanisms: reminiscence and sensory stimulation" (Whear 703). These spaces provide opportunities for residents to perform meaningful activities that can offer stimulation and relaxation within the care facility, as people who are "outside and active can still maintain an active interest in their environment, and their cognitive abilities decline slower"(Williams). Medical research highlights that exposure to natural light in the outdoor environment is critical, as "nature based experiences were found to be therapeutic, restorative or healing, contributing to the emotional, psychological and spiritual intangibles of well-being" (Chalfont 57). Exposure to the outdoors through "exercise and taking vitamin $D$ have been shown in numerous studies to be extremely important in maintaining good physical health, in preventing falls and also in maintaining cognition" (Feddersen 150). Therefore a design that incorporates light stimulation to emphasize daily rhythms of light within indoor and outdoor environments, offers opportunities to treat circadian misalignment to reduce the severity of disruptive sleeping behaviour (Figure 2.4).

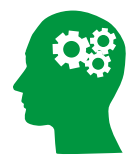

Light Stimulation

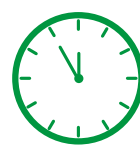

Circadian Lighting

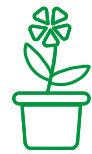

Outdoor Spaces

2.4 Light therapy strategies 


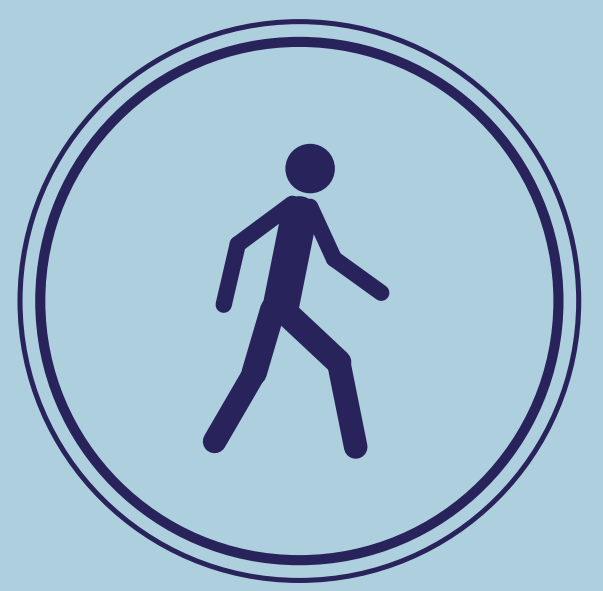

Movement as therapy 
Although wandering is a key symptom associated with dementia, it offers a potential opportunity to reduce disruptive sleep through the act of movement. Medical evidence suggests "a combination of bright light therapy and walking has shown additional benefits for attention, memory and orientation in residents and reduced caregiver burden"(Desiree). Circulation paths which integrate alongside therapeutic elements such as environmental alterations and social activities (Figure 2.5), help create a safe and enjoyable environment for staff and residents while providing meaningful programming to people with dementia (Cipriani 139). As movement allows for cognitive stimulation of the brain, this improves the wellbeing of those affected by dementia, so "prompting safe walking rather than preventing wandering aims to balance a person with dementia's need for autonomy with the need to minimize risk." (Ibid 139).

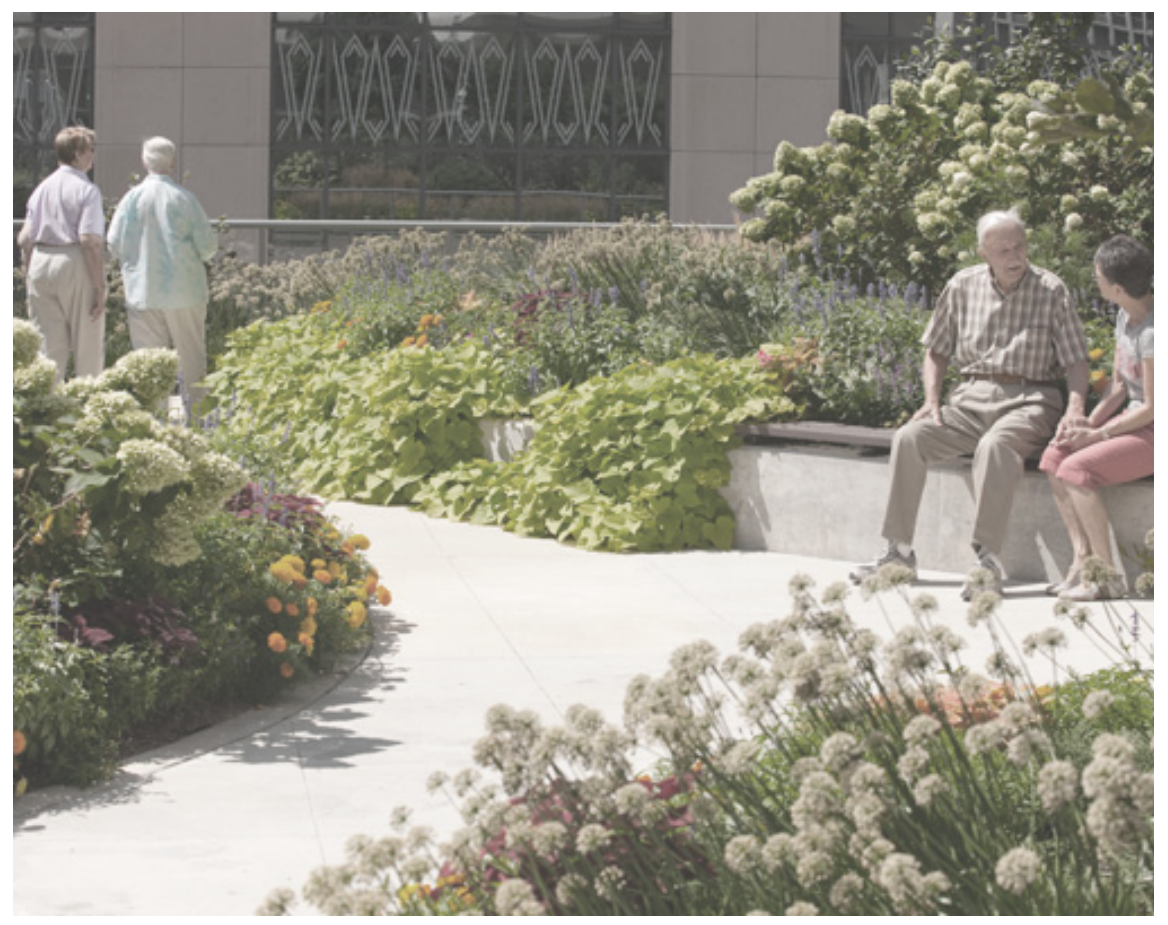

2.5 Circulation routes integrated with outdoor spaces 


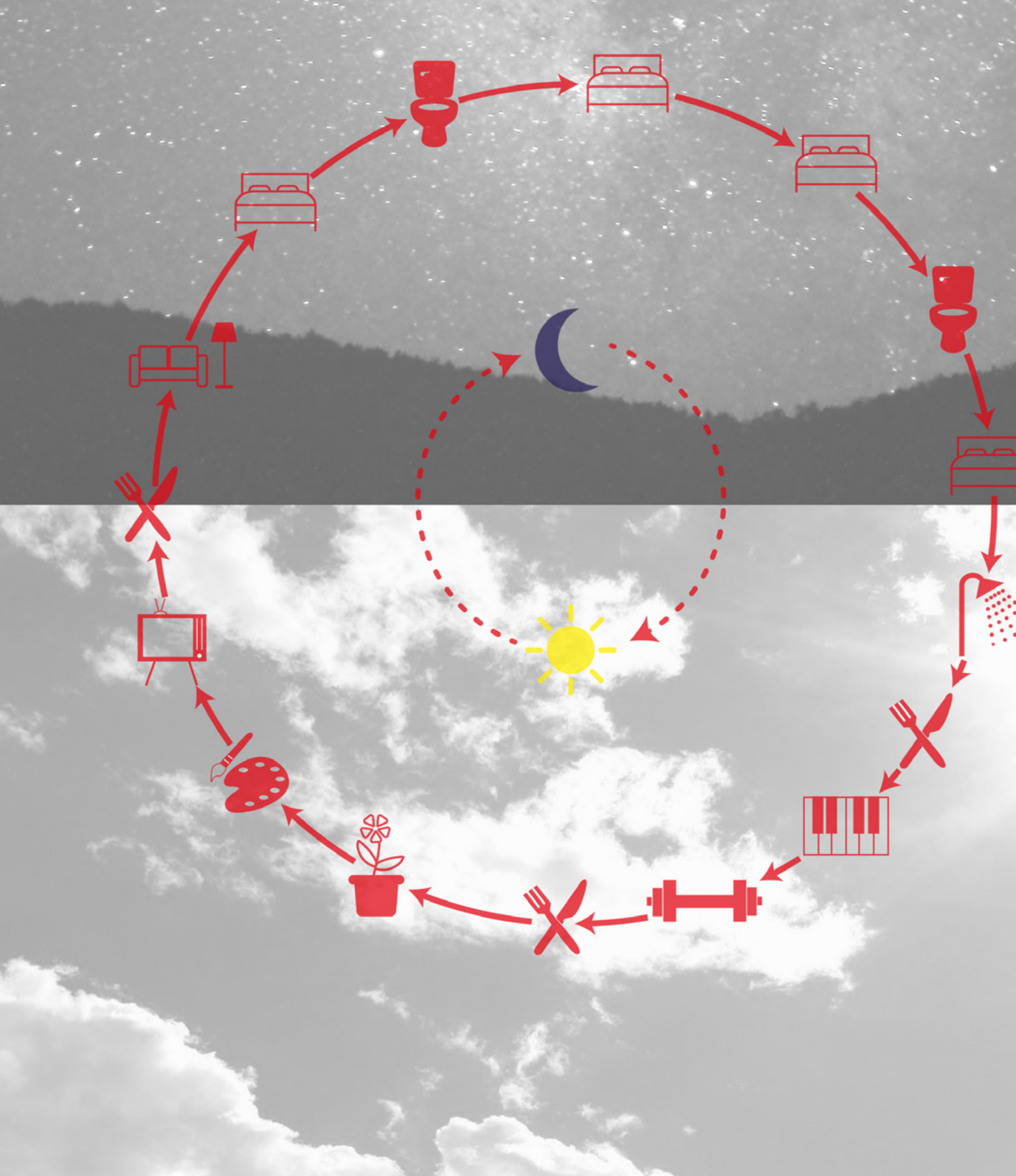

2.6 Programmatic circulation corresponding to circadian rhythms 
Circulation routes that offer visual and functional cues can improve independent orientation for those affected by dementia. The use of external stimuli through windows can aid to facilitate both non-cognitive and seasonal orientation (Ibid 10). Knowles identifies similar notions of movement within the home related to external stimuli from the sun, highlighting "migration within the dwelling as a primary mode of adaption to climate" (Knowles 37). Furthermore, Knowles identifies how comfort levels related to lighting conditions allow for seasonal migration within the home, the spatial organization of the home supporting adaptive migrations for different times of the day or year (Knowles 40). By integrating movement patterns alongside natural light patterns, circulation can be used to achieve therapeutic routines, allowing "multi-sensory walking loops that enable residents to meet their need to move, as it occurs" (O'Brian 15). As natural light rhythms represent a familial daily routine, integrating movement alongside this daily pattern offers opportunities to strengthen the relationship between these two therapeutic interventions (Figure 2.6).

To allow for movement within a dementia care facility, evidence based design have identified specific design principles to help facilitate movement efficiently and safely for residents. "Plan configurations can support or impede orientation, having a significant impact not only on the quality of life for residents, but also staff workload and also operational costs " $(O$ 'Brian 6). Recent evidence suggests that orientation strategies are based upon implicit rather than explicit memory. These strategies can be implemented through "the use of repetitive stimuli to produce a non-cognitive response. Designs could include the use of looping circulation paths, a unique sequence of unobtrusive route identifiers and nodes with clear lines of sight to the next destination" (lbid 11). Although safety is important when facilitating movement, a balance is necessary to promote autonomy for residents too, as the "ability to function independently has been connected to improved sense of self and of wellbeing among residents of aged care facilities "(Ibid 12). A design that promotes movement by facilitating independent orientation for residents through a clearly legible environment, offers opportunities to re-interpret the act of wandering as a form of sleep therapy rather than a symptom (Figure 2.7).

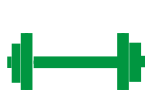

Promote Exercise
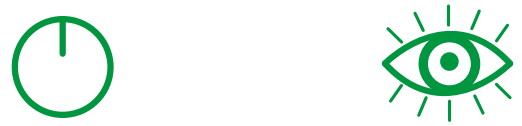

Independent Orientation
Legibility 


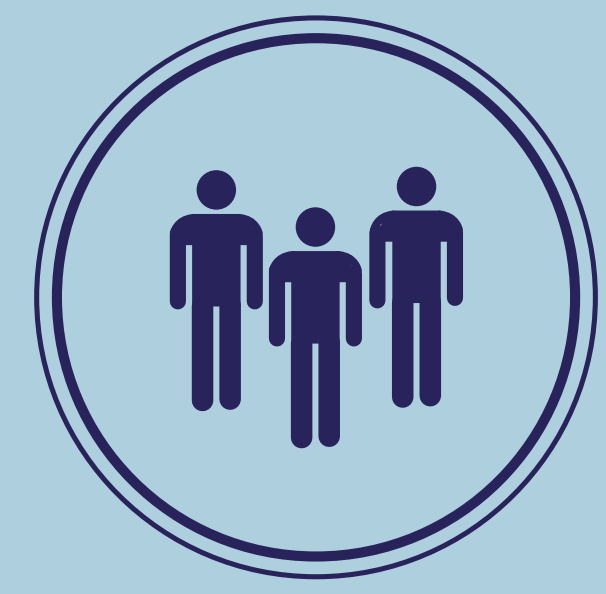

Community as therapy 
Improving social interactions within institutional facilities has benefits towards improving the sleep behaviour and wellbeing for people affected by dementia. Medical research has highlighted that dementia residents who participated in meaningful social activities showed a significant decrease in daytime napping, corresponding to improved day/night sleep ratio and an improved circadian rhythm (Richards 1515). Instigating community integration within the institutional environment can reduce the sense of isolation felt among residents, creating opportunities for stimulation and social interactions (Figure 2.8). Staff and family "can mistakenly conceptualize dementia as a loss of self, resulting in missed opportunities for continual social connection. However the needs and desires of residents are often no different to individuals who do not have dementia" (O'Brian 36).

Integrating the dementia care facility within the community aligns with Rafael Moneo's critical reflection of architectural typology. The perception of type as a "frozen mechanism" that denies change and emphasizes automatic reproduction refers to stability in society, reflected in the activities that are imitated by the architecture (Moneo 27). Moneo identifies that the most intense moments in architectural development are those when a new type appears, often arising from new techniques or changes within society (lbid 28 ), these changes in society generate a "frame" in which typology can be manipulated (Ibid 27). By instigating the typology as a point of interest within the community, increased social exchange within the respite facility can allow for improved cognitive stimulation for residents and reduce the sense of isolation often experienced within institutional environments.

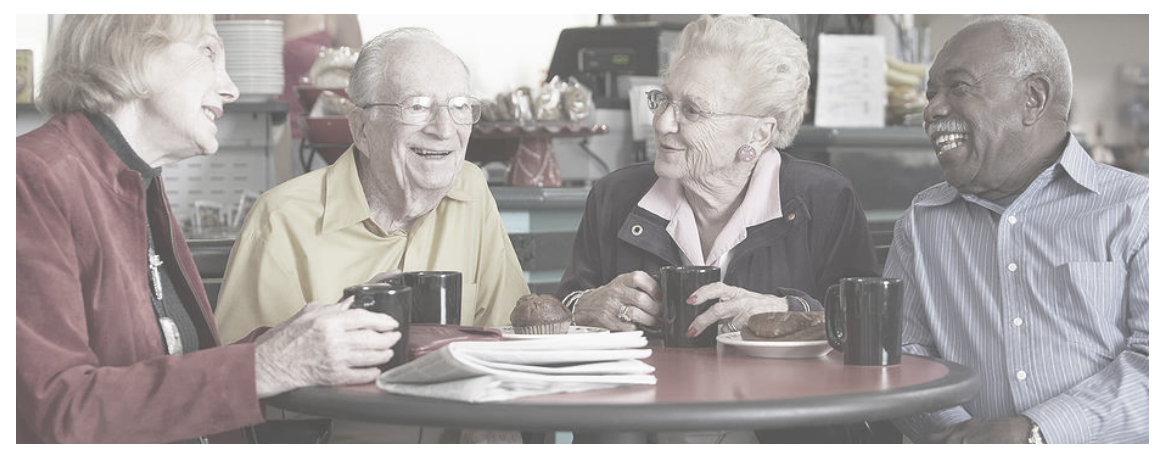

2.8 Social activities allowing for cognitive stimulation 


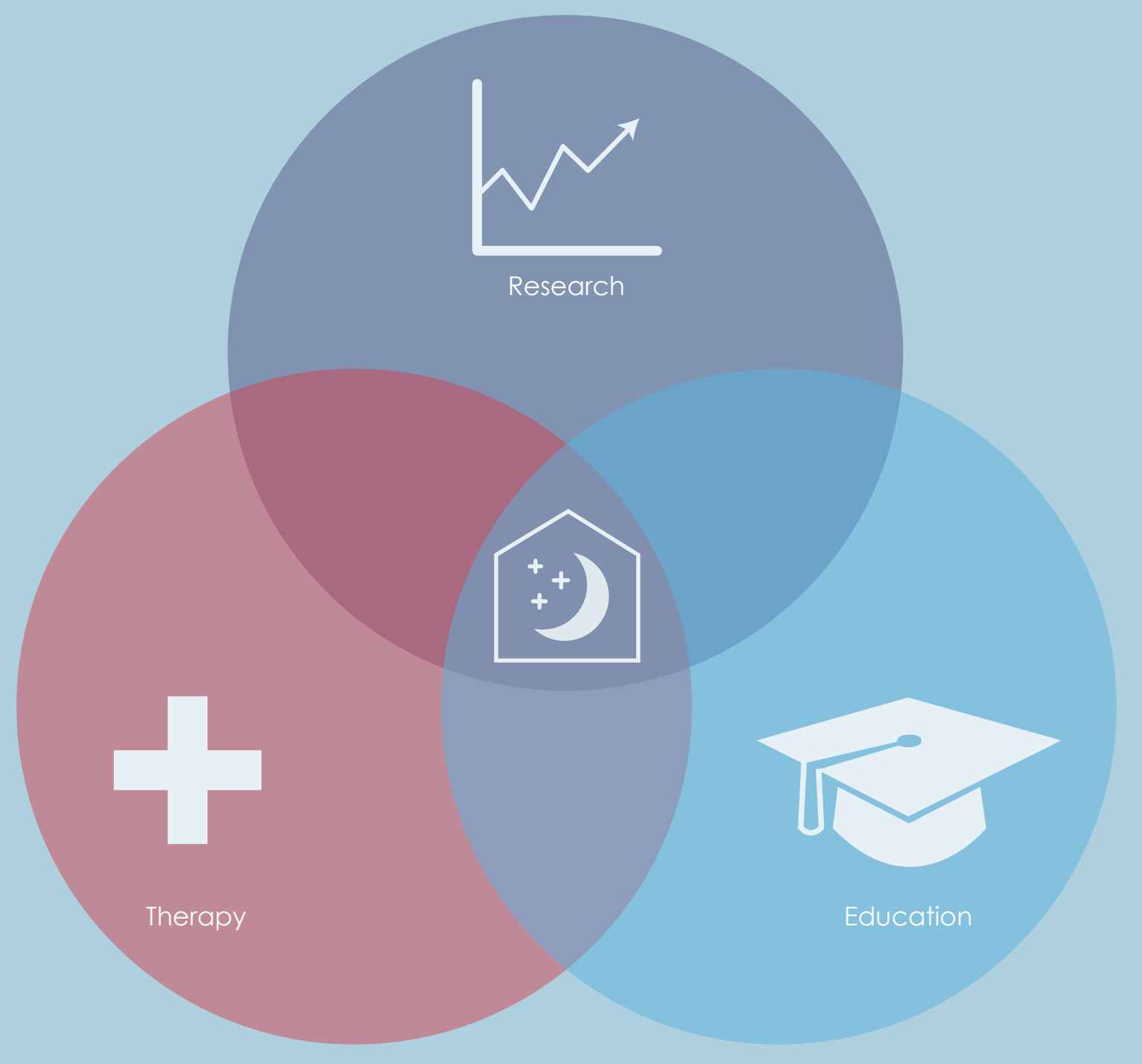

2.9 Integrating auxiliary programs for mutual benefits 


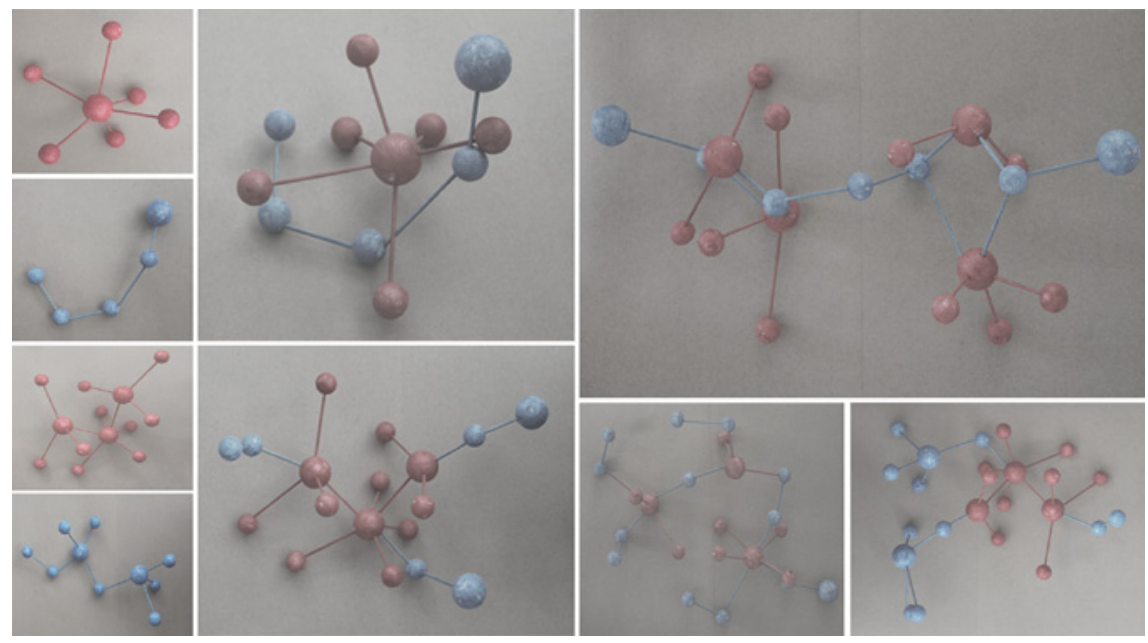

2.10 Parti models testing disprogramming strategies

Integrating auxiliary programs alongside respite care allows opportunities to connect the community within the institutional environment. The integration of education and research programs offers an active approach towards improving sleeping behaviour of residents, families and caregivers (Figure 2.9), as evidence showed that the inclusion of caregiver-specific education with a systematic sleep-training program improved caregiver sleep quality significantly as a result of treatment (McCurry 127). The interactions between education and respite programs provide a method to manipulate the dementia care typology through programmatic variation. Architectural theorist Bernard Tschumi suggests that "architecture should cease to separate these categories and instead merge them into an unprecedented combination of programs and spaces" (Tschumi 194), allowing for the exchange of these programs to influence one another (Figure 2.10). Tschumi identifies this exchange as disprogramming, a strategy "whereby a required spatial configuration of program $A$ contaminates program $B$ and B's possible configuration"(Tschumi 195). By interacting the behavioural and spatial qualities associated with each program, the act of condensing these programs into a single facility can help to improve the sense of wellbeing for those affected by dementia. This will provide the "possibility to actively contribute and shape the social environment, to interact and speak with other people, and to feel jointly responsible" (Feddersen 57). The integration of auxiliary programs within a respite facility offers the potential for increased social interaction amongst residents, as well as creating a point of interest for integration within the wider community (Figure 2.11).

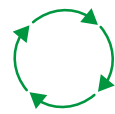

Community Integration

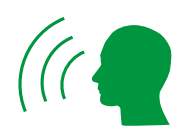

Social Spaces

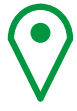

Point of Interest

2.11 Community therapy strategies 


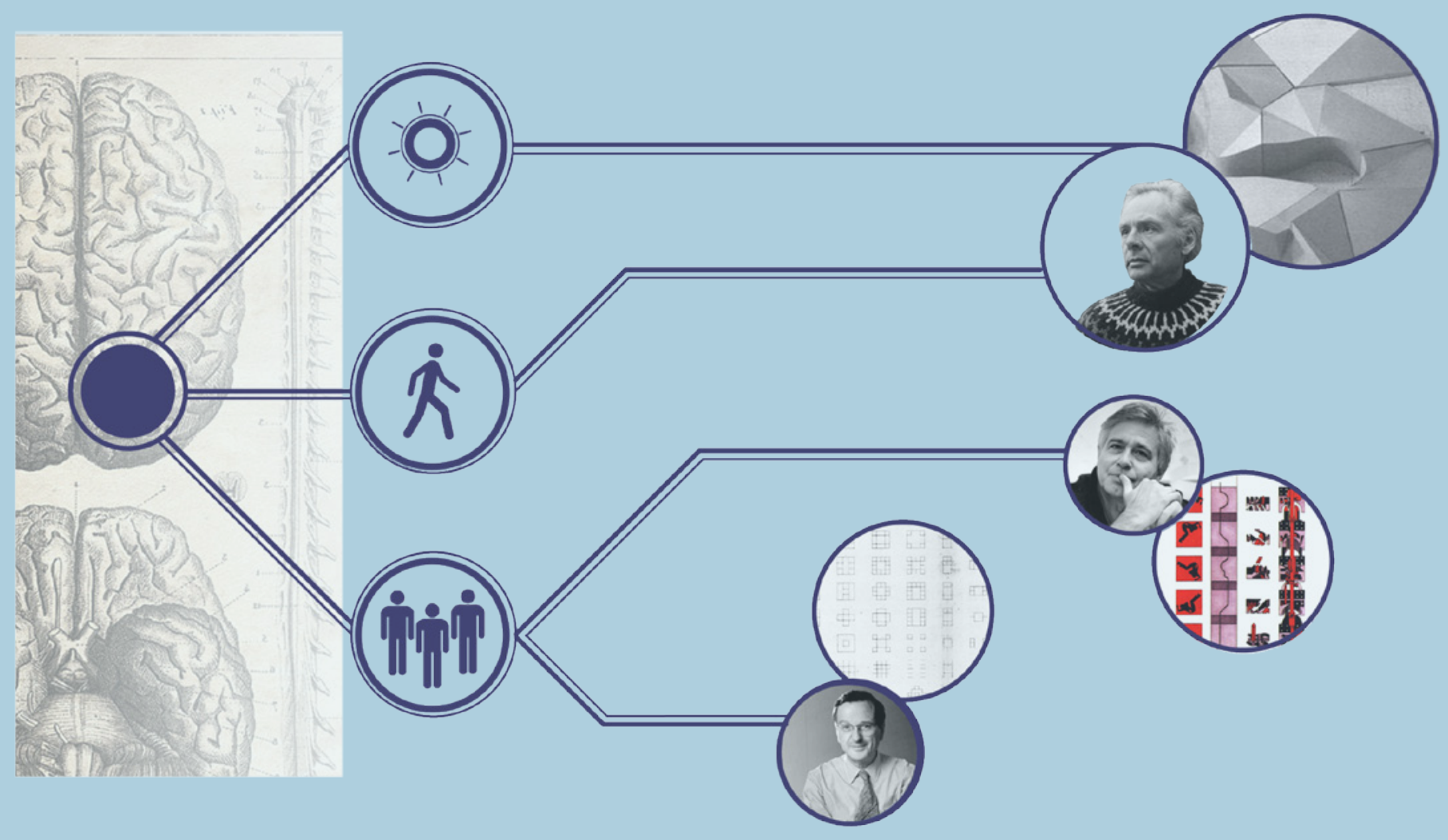

2.12 Linking medical evidence with architectural theory 


\section{Summary + Reflection}

This literature review has highlighted the therapeutic potential of the environment to reduce disruptive sleeping behaviour of residents during respite care. The integration of medical evidence alongside architectural theory (Figure 2.12) allowed for the development of architectural strategies to create a design criteria that includes:

1. Light therapy to stimulate residents and improve their circadian rhythms through the use of outdoor spaces and exposure to light at specific times of the day.

2. Promoting movement through circulation layouts that allow for independent orientation and a clearly legible environment to improve a residents sense of autonomy as they wander.

3. Integration of community facilities such as education and research programs that instigate social exchanges for residents and create a point of interest within the wider community.

The integration of light, movement and community can help to facilitate a stimulating environment that reduces the effect of disruptive sleeping behaviours during respite care. By alleviating this problematic symptom, the opportunity to reduce sleep deprivation of the caregiver and the overall burden of care can help to prolong home care for thousands of families throughout New Zealand. 


\section{Project Review}

The following analysis applies the design criteria identified within the literature review alongside international and local precedents. Evaluating the appropriateness of design methods used towards achieving the design criteria will help to inform the design led process and strengthen the effectiveness of the built environment towards sleep therapy. 


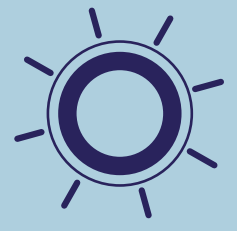

$3 / 3$

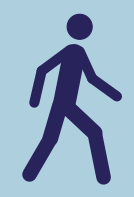

$3 / 3$

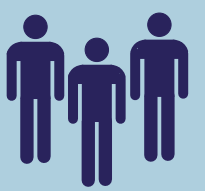

$3 / 3$

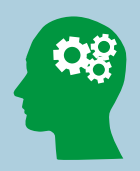

Light Stimulation

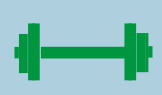

Promote Exercise

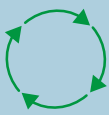

Community Integration

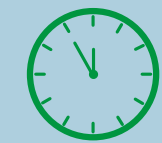

Circadian Lighting

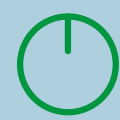

Independent

Orientation

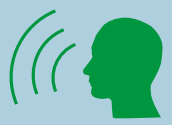

Social Spaces

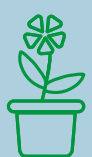

Outdoor Spaces

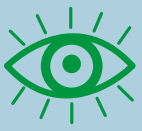

Legibility

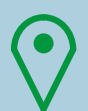

Point of Interest 


\section{Method Of Analysis}

The literature review identified key architectural strategies to integrate into the design of a respite facility that can improve the sleeping behaviour for those affected by dementia (Figure 3.2). The design of each precedent has been assessed against light, movement and community and then simplified into diagrammatic form to compare the strengths and weaknesses of these architectural strategies for each precedent (Figure 3.3). As there are limited examples of respite facilities that are specifically designed to improve sleeping behaviour for residents, the analysis extended to include full time dementia care facilities as well.

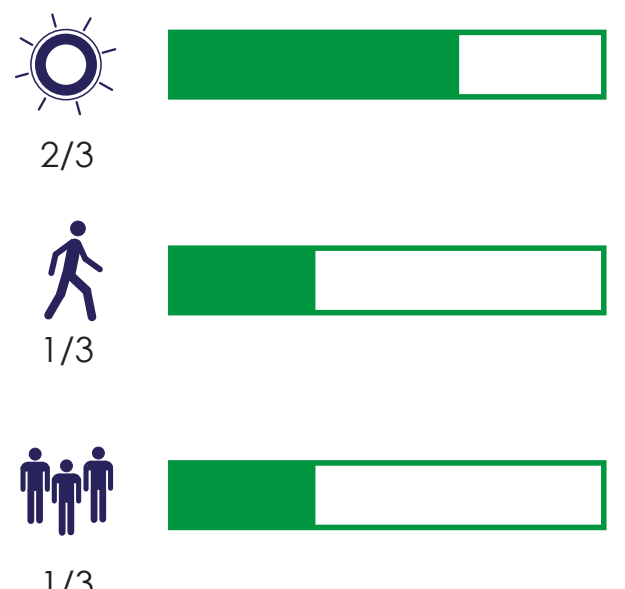

$1 / 3$

3.3 Design performance diagram 
De Hogeweyk

Weesp, Netherlands

Molenaar \& Bol \& Van Dillen

\section{Strengths}

Outdoor spaces act as primary circulation routes to integrate mobility alongside daylight exposure.

Outdoor courtyards with a variety of seating arrangements allows for opportunities for social interactions amongst residents

Inward focused design creates a secure environment for residents with care staff acting as café staff, hairdressers and other shop assistants to create an artificial community to reduce sense of isolation

\section{Weaknesses}

Monotonous building forms and cladding may cause confusion and disorientation amongst residents

The inward focused design creates a lack of opportunities for social engagement amongst Weesp community, maintaining a sense of detachment from wider community
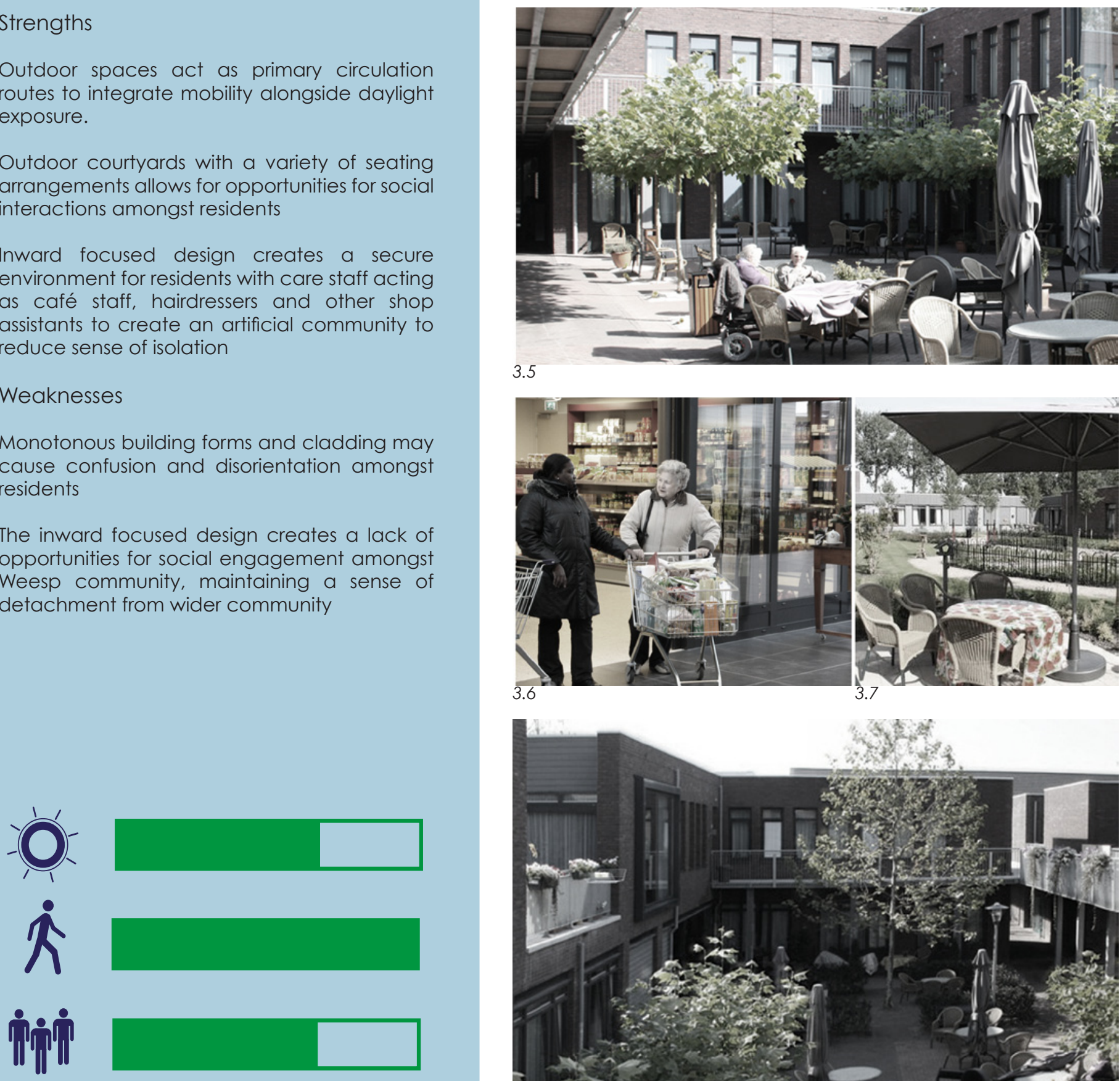

3.5
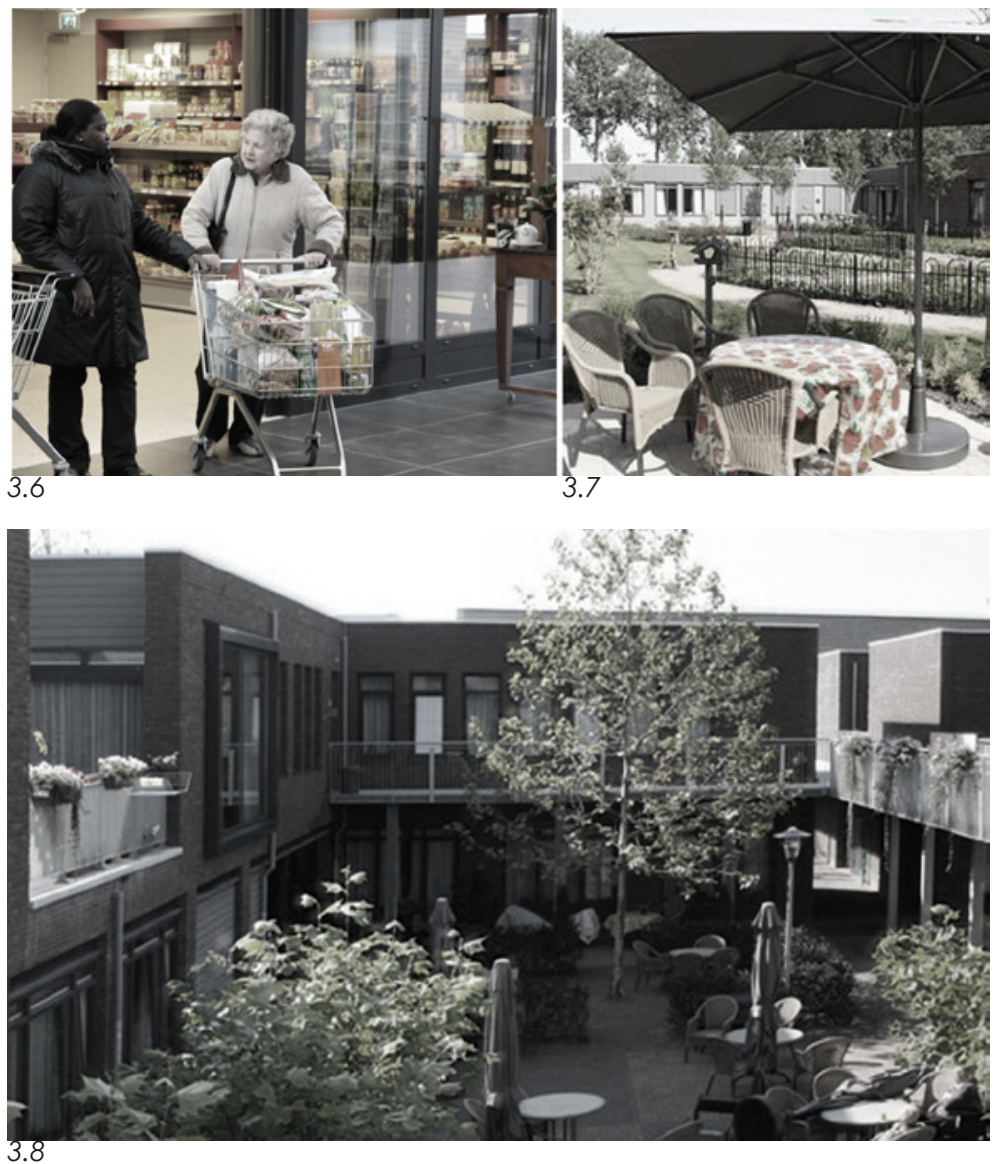

Design performance

Outdoor social spaces

Supermarket

Therapeutic garden

Courtyard space

Outdoor circulation

3.10 Districts as environmental indicators

3.11 Orientation strategies along boulevard 

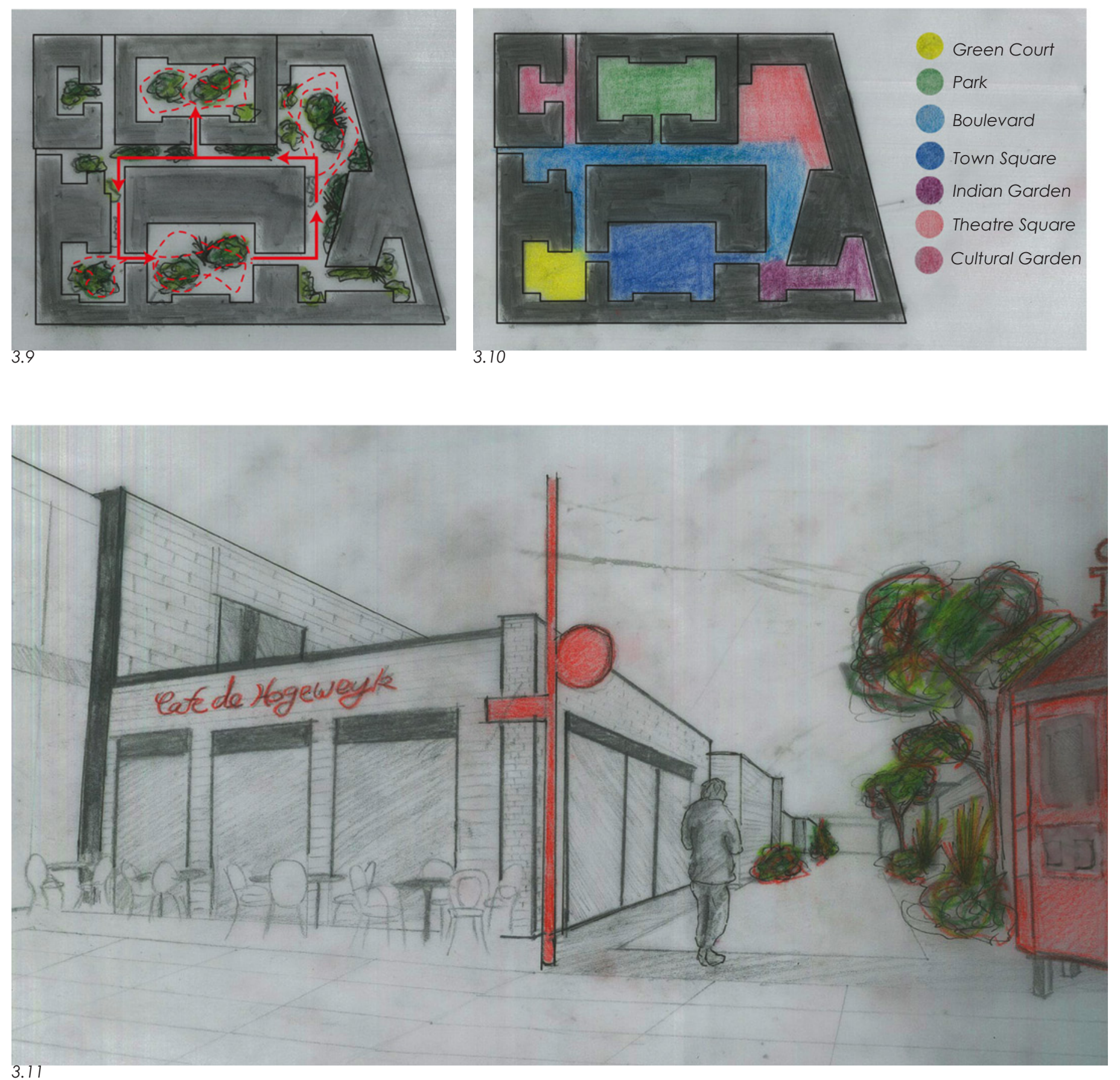
The Five Gardens

Lier, Norway

\section{C.FMØller}

\section{Strengths}

Roof profile ensures maximum natural light for residents and disrupts the monotonous appearance of the large building forms

Design recognises the courtyard typology as a tool to promote wandering for residents, allowing for efficient movement to reduce disorientation and confusion amongst residents

Green atriums ensure residents are exposed to light and seasonal changes for multisensory stimulation

\section{Weaknesses}

The verticality of the three-storey structure may be physically difficult for residents, as staircases and elevators for circulation can impede movement for residents

A limitation of the courtyard typology is its inward orientated design, limiting the public interface between residents and the wider community
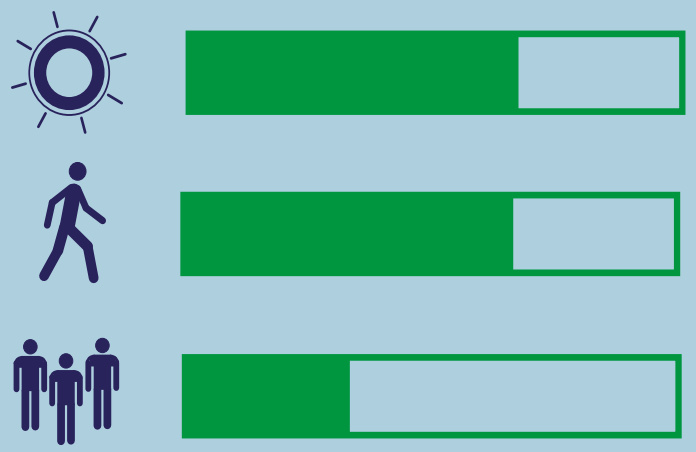

3.12

\subsection{Design performance}

3.13 Entrance

3.14 Bedroom

3.15 Green atrium one

3.16 Green atrium two

3.17 Circulation and outdoor space

3.18 Roof profile efficiency for natural light

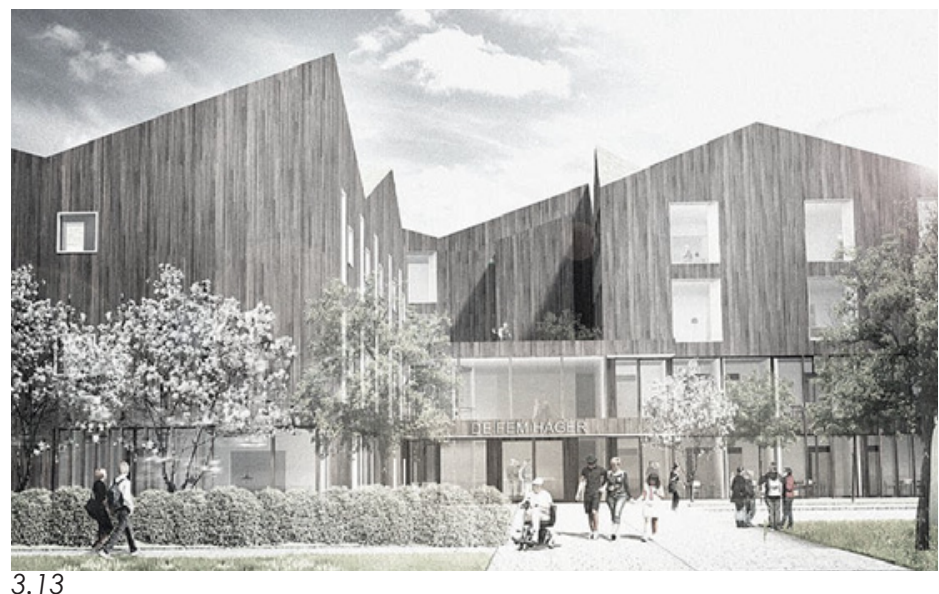

3.13
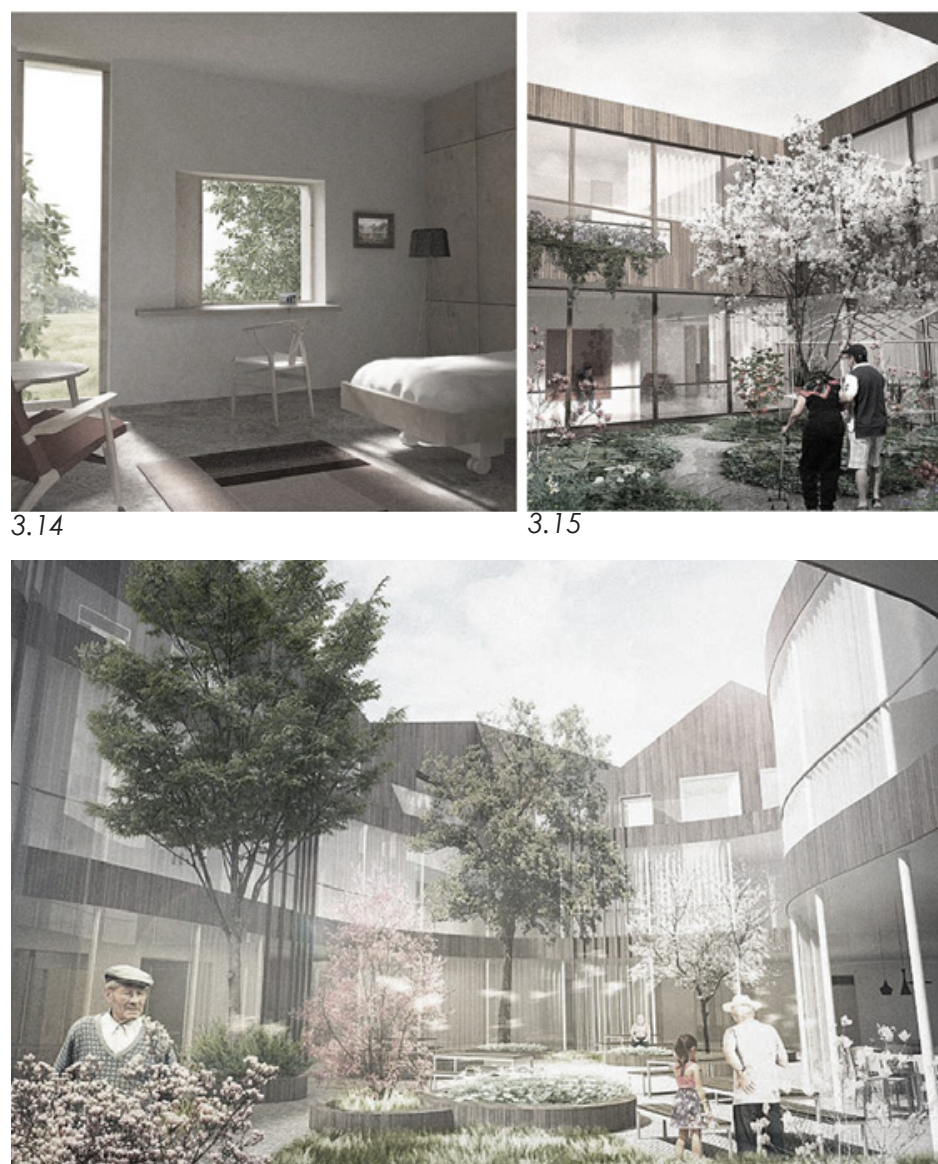

3.16 

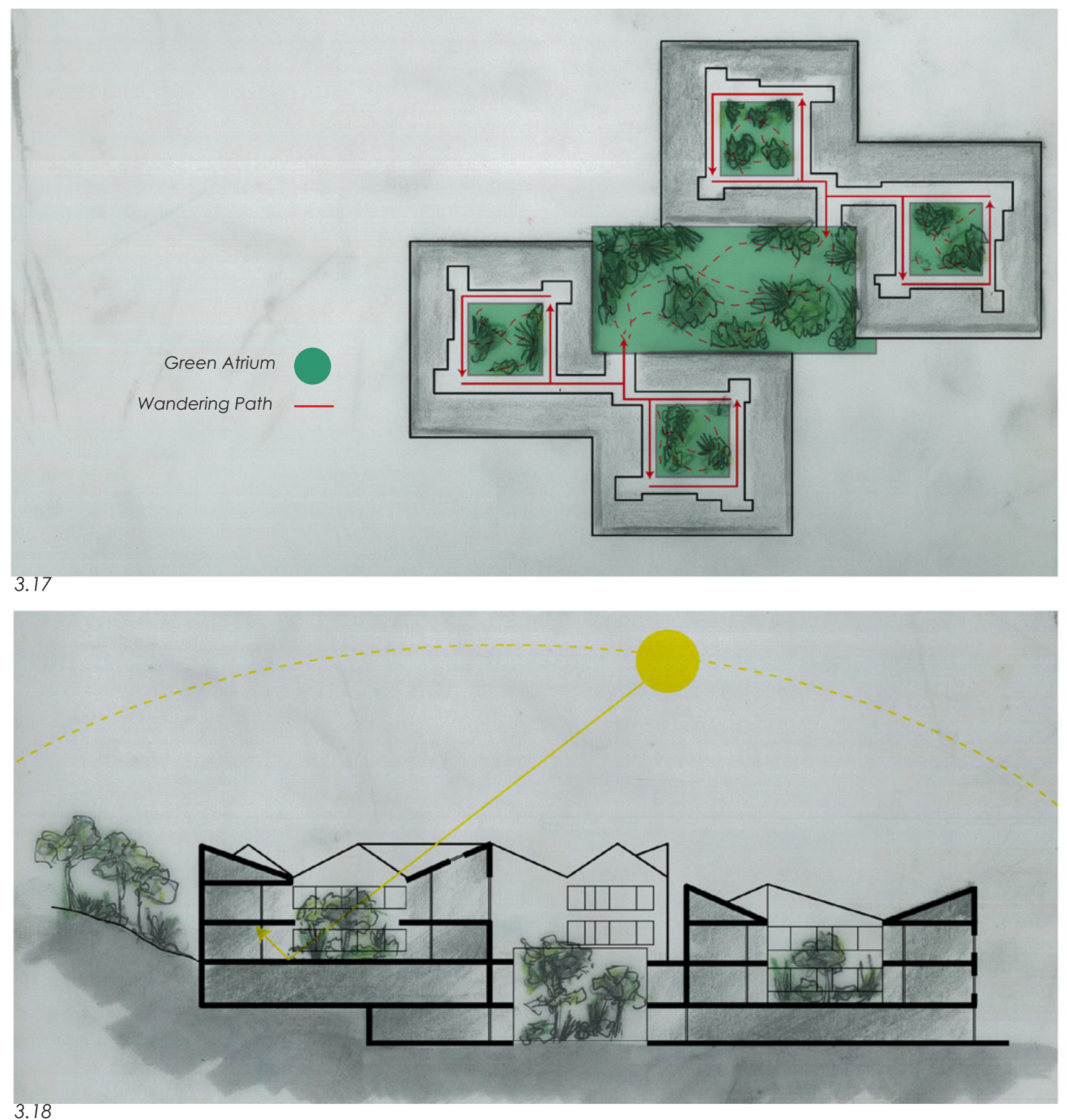
Alzheimer's Respite Centre

Dublin, Ireland

Niall McLaughlin Architects

\section{Strengths}

Programs are arranged according to natural light patterns on the site, the movement of the sun utilized to facilitate orientation and circulation patterns for residents within the building

Gardens are orientated in different directions to account for sunlight variation during the day and emphasise circadian movement amongst residents within the building

Ceiling profile maximises natural light within the interior space to stimulate residents and provide light therapy throughout the day

\section{Weaknesses}

Ceiling height to achieve natural light exposure reduces domestic quality of spaces

Lack of colour or tint in glass panels that connect to floors may cause injury to residents, as poor vision and lack of spatial awareness could cause residents to walk into windows
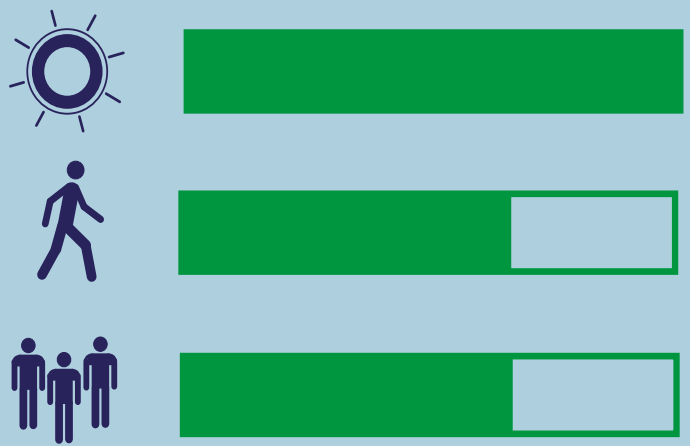

3.19
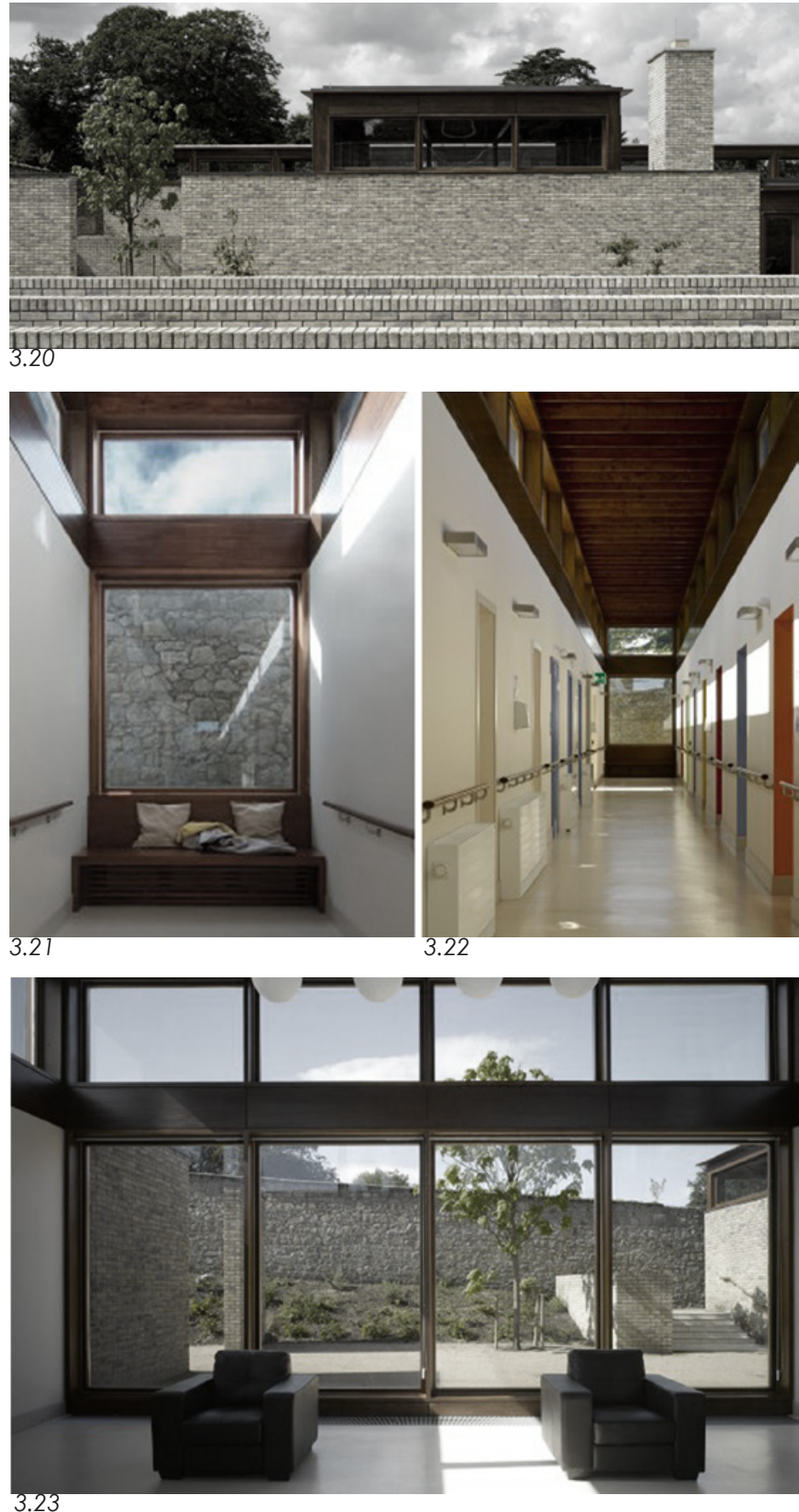

\subsection{Design performance}

3.20 Box lantern external perspective

3.21 Seating area

3.22 Corridor space

3.23 Living room

3.24 Wandering paths and courtyard

3.25 Box lantern light diagram 

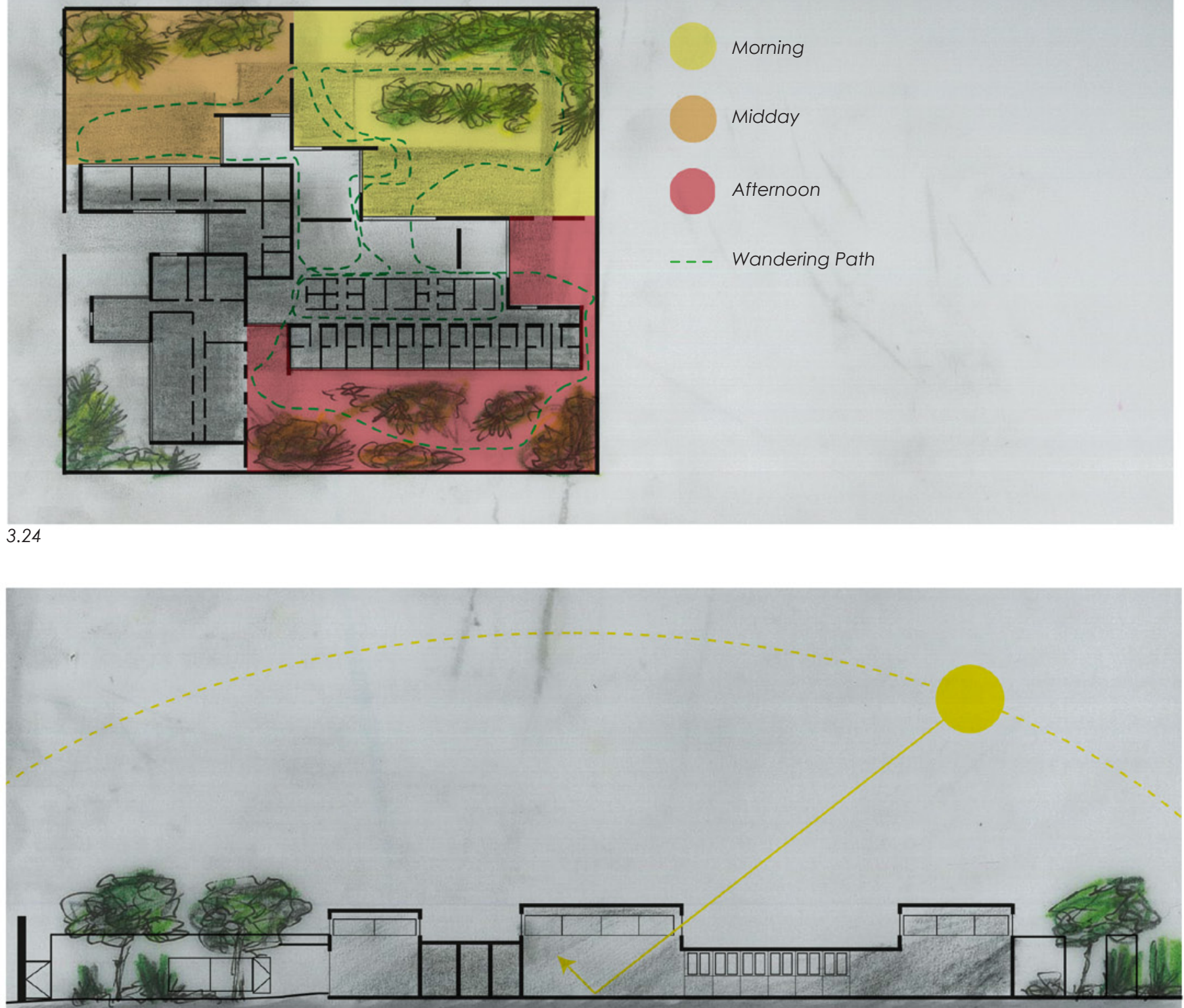


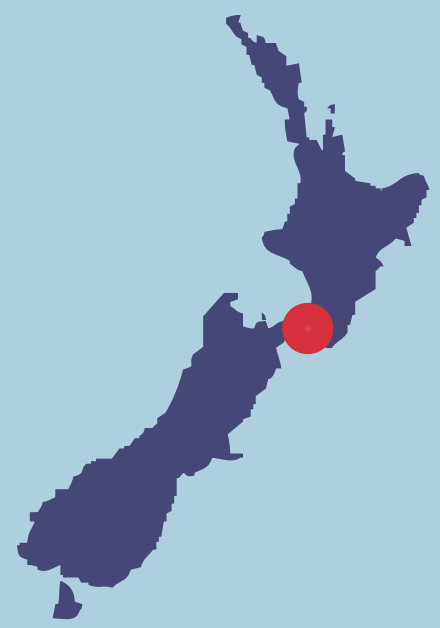

\section{Local Context}

Field trips and informal discussions with carers, nurses and medical processionals in the Wellington region provided a valuable insight towards the strengths and weaknesses of design methods adopted within care facilities. The analysis was also critical in identifying local initiatives utilizing environmental therapy for sleep in the form of the Massy University Sleep/ Wake Research Centre (Figure 3.26). 


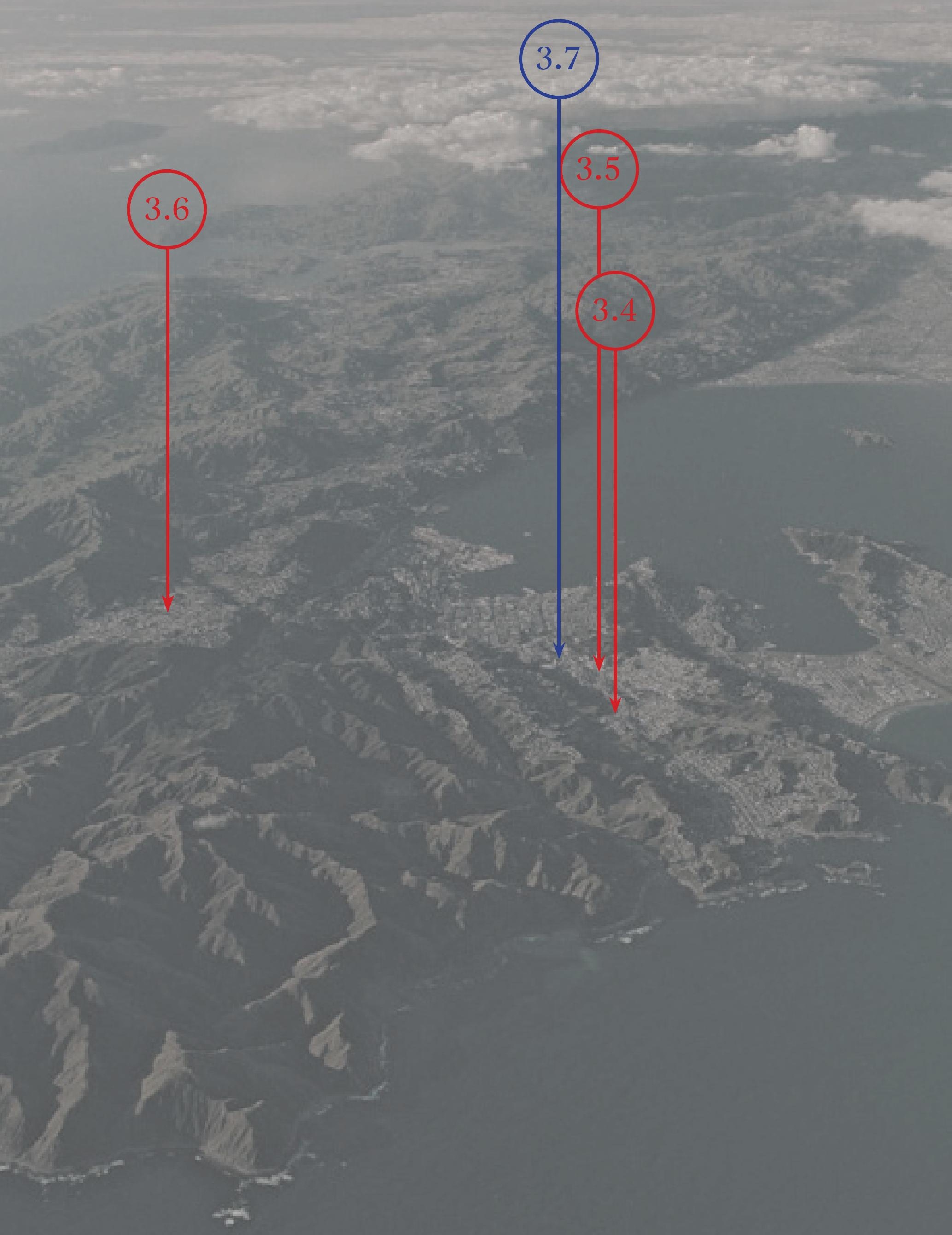


Village at the Park

Newtown, Wellington

\section{Strengths}

Windows orientated east in living and dining room spaces to provide morning light stimulation for residents

Dementia unit is integrated with retirement village and kindergarten to allow for community integration on site.

\section{Weaknesses}

Circulation is disrupted by outdoor courtyard, with a $u$ shaped building form causing disorientation amongst residents due to dead ends

While the position of the bed orientates the resident towards outdoor space upon awakening, it lacks visual orientation towards toilet to deal with incontinence during the night

Visual cues on ceiling to facilitate orientation for residents have limited effectiveness, as primary visual orientation for residents is directed towards the floor
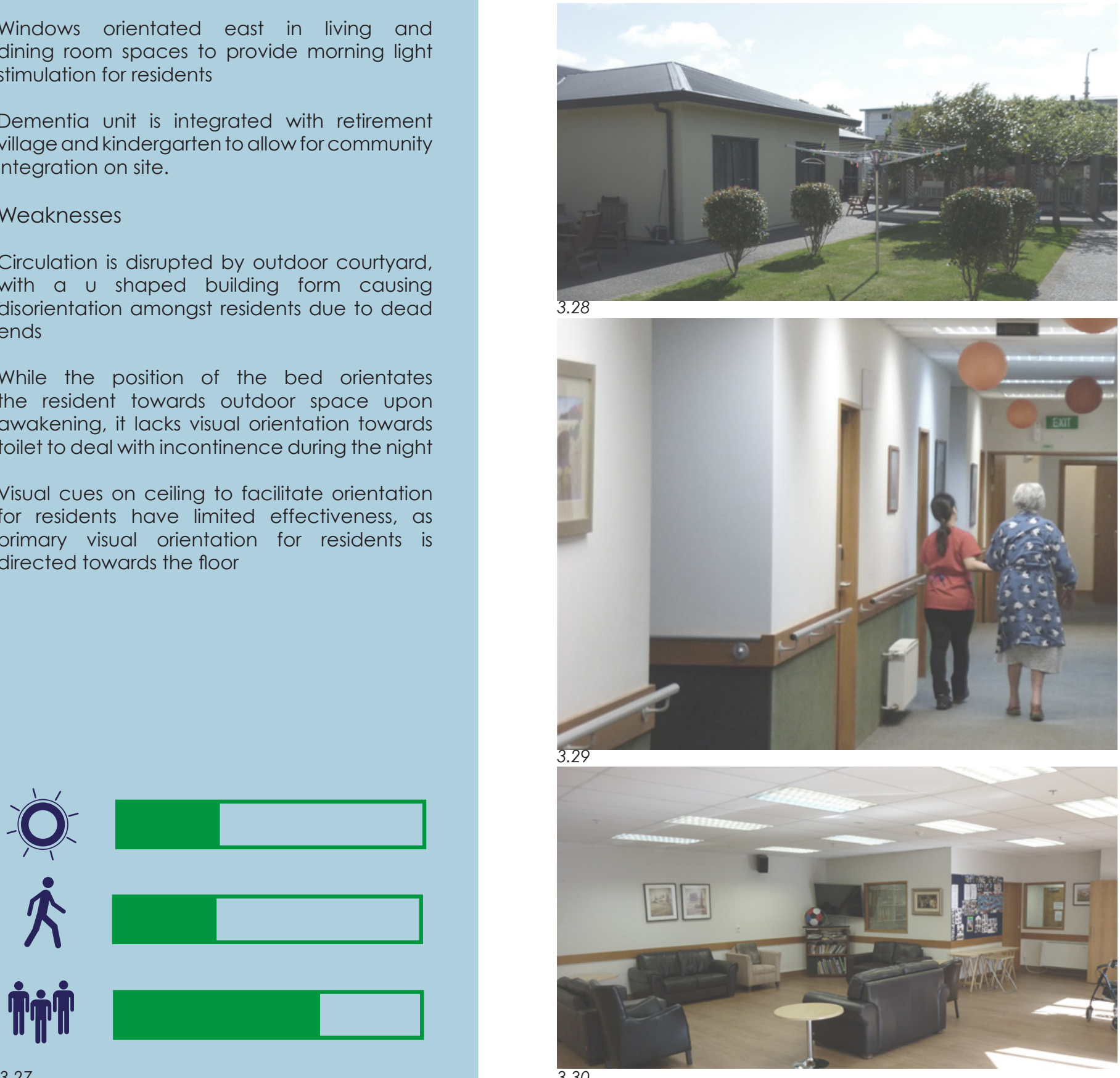

3.33 Ineffective position of indicators 

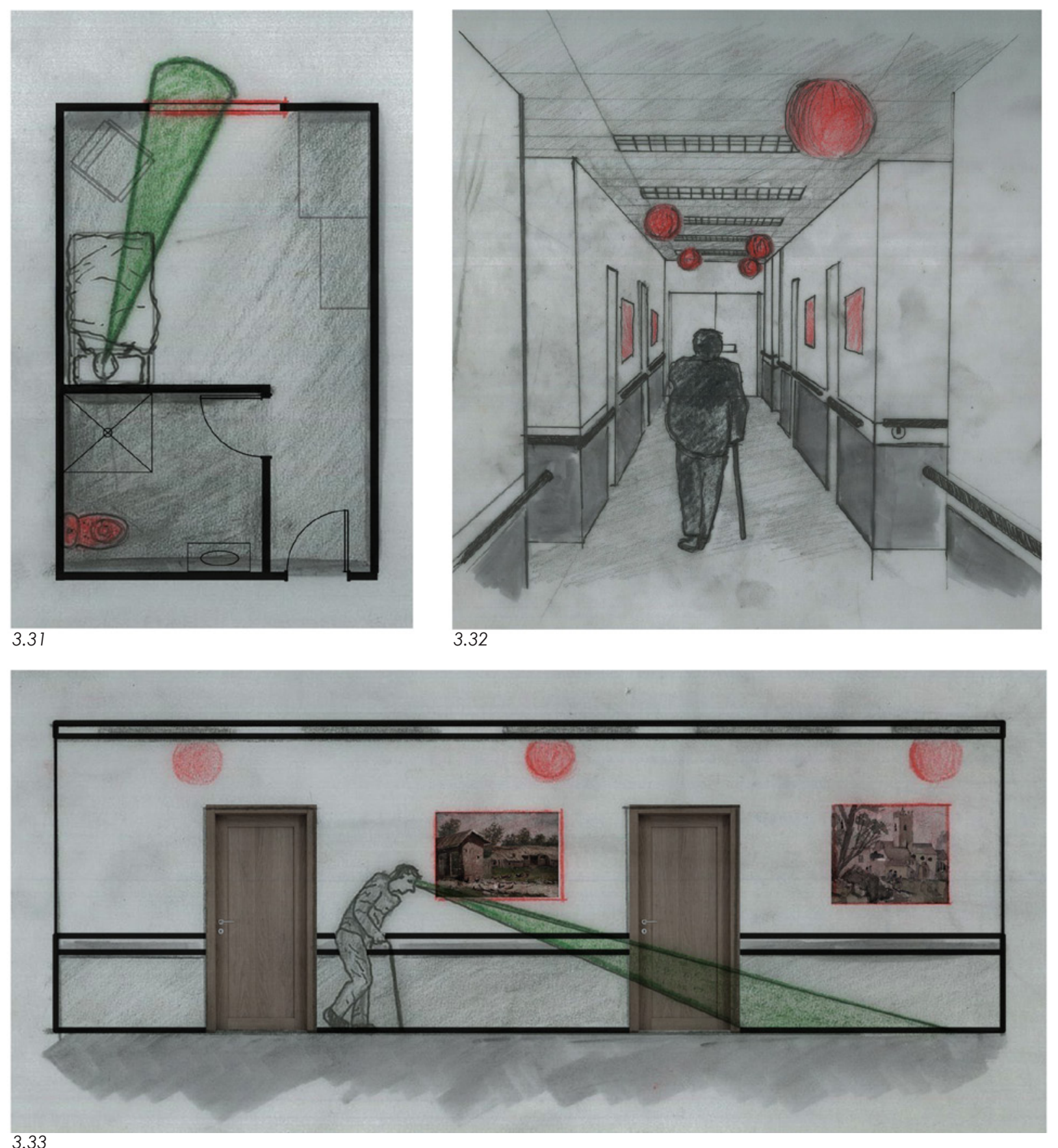
Te Hopai

Newtown, Wellington

\section{CCM Architects}

\section{Strengths}

Staff training facilities and auxiliary hospital program strengthens its appeal for use within existing medical community

\section{Weaknesses}

Exterior form and entrance mimics medical aesthetic of similar buildings on site, reducing it as a point of interest within the community and domestic quality to residents and family visiting building

Predominant use of artificial light within interior spaces reduces effect of daily sunlight patterns and seasonal variation for residents

Outdoor courtyard spaces remain stark and unstimulating for residents, with scale of garden space lacking opportunities for multisensory stimulation and outdoor activities
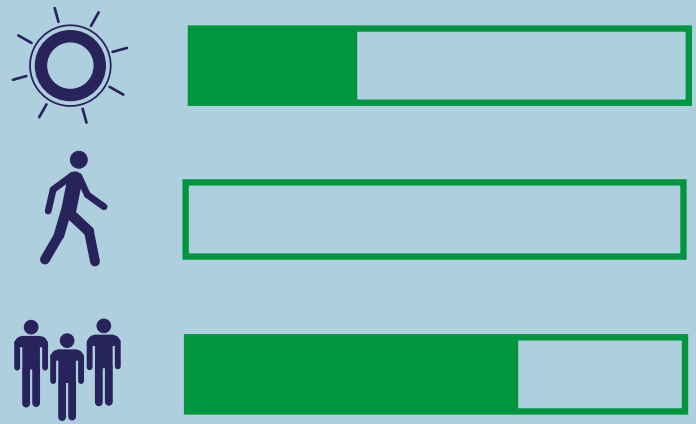

3.34

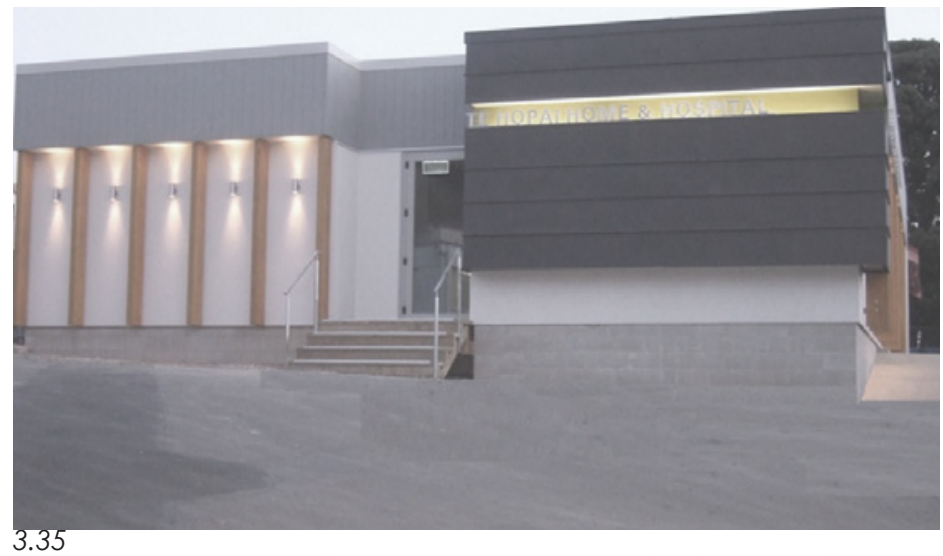

3.35

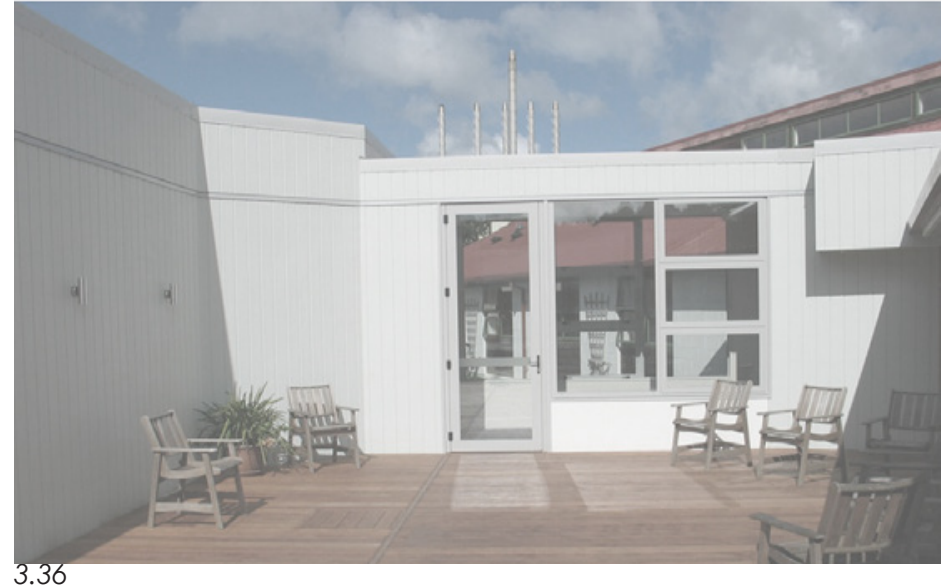

3.36

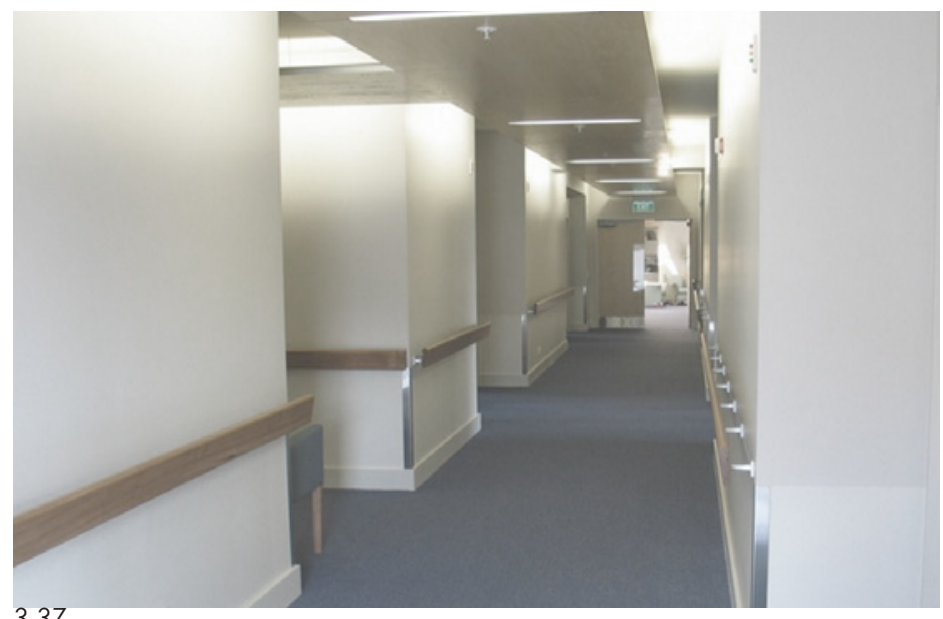

3.37 

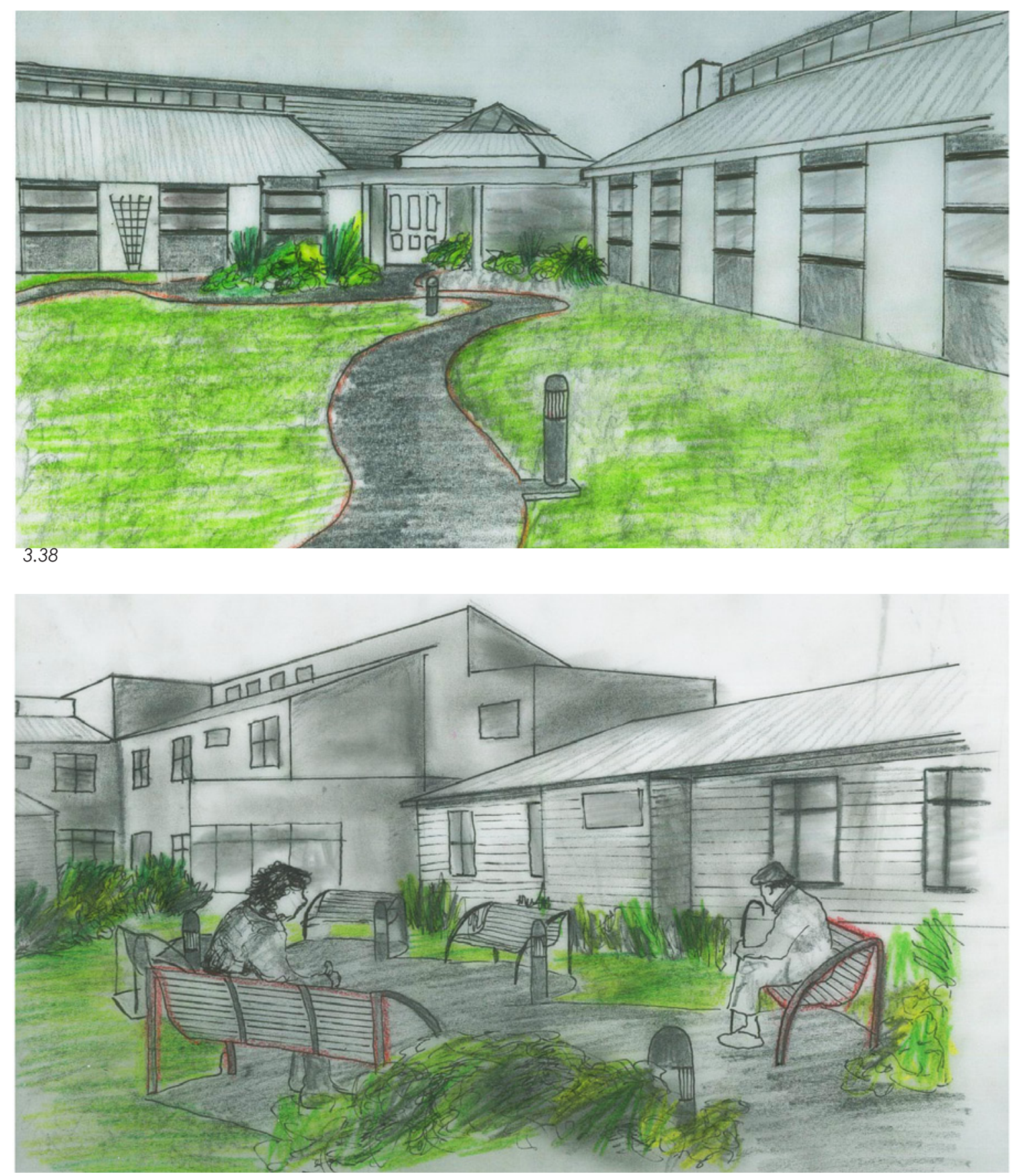


\section{Sprott House}

Karori, Wellington

\section{Strengths}

The glass pavilion addition allows for an enclosed space to improve access to natural light during different seasons

Windows and small areas for rest along circulation route provide visual and functional cues to improve movement within facility

Auxiliary education and conference room spaces provide opportunities for learning and teaching on site, proving greater community involvement within the facility

\section{Weaknesses}

The scale and transparency of windows may cause injury for residents as dementia causes disruption of spatial awareness

Outdoor spaces lack a suitable multisensory environment, with a lack of vegetation and artificial turf reducing quality of the outdoor space.
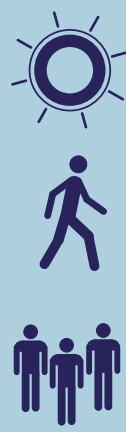

3.40
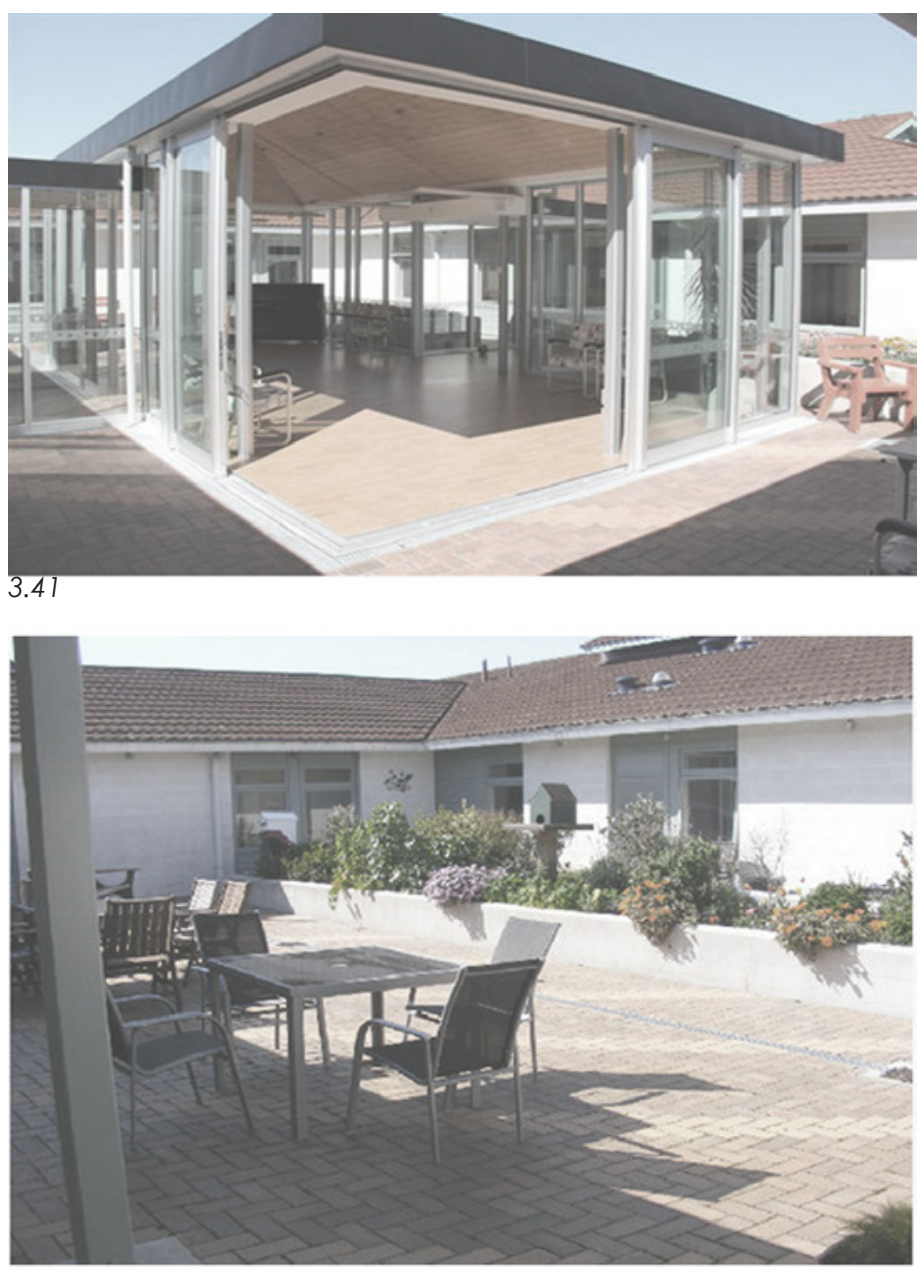

3.42

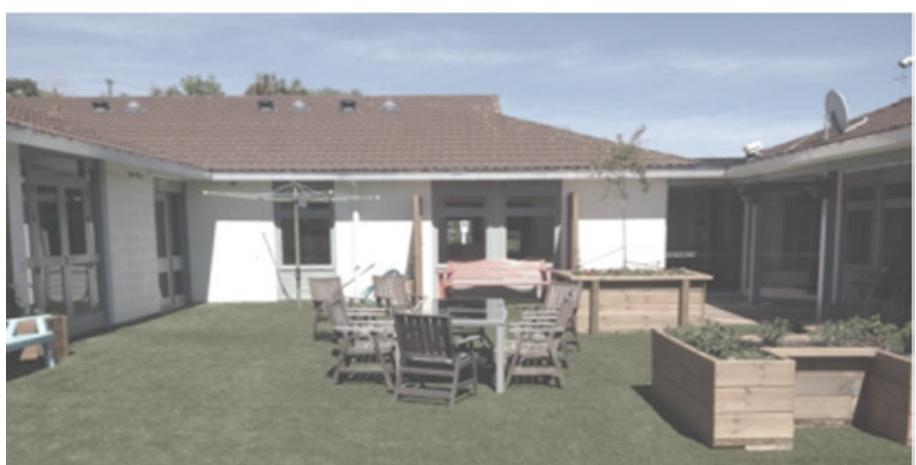

3.43
3.40 Design performance

3.41 Glass pavilion

3.42 Courtyard space

3.43 Courtyard space

3.44 Inappropriate window scale

3.45 Improving accessibility 

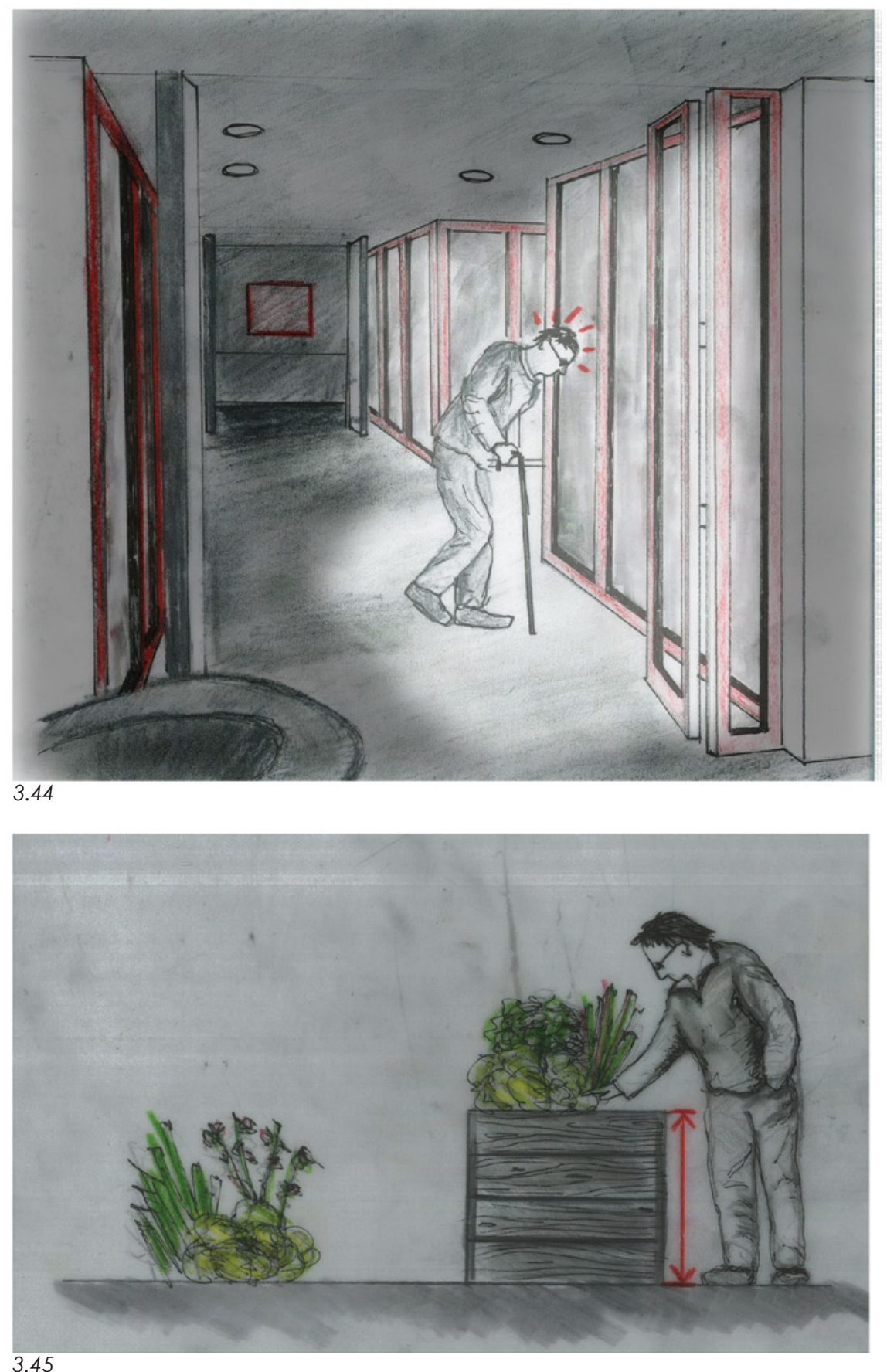
Massey University Sleep/Wake Research Centre Newtown, Wellington

A current practice towards monitoring, assessing and providing treatment for disrupted sleeping patterns is conducted within a 3 bed time isolation facility, a room within a room layout that is completely devoid form the external environment. Patients remain in the facility for durations of up to a month as researchers monitor and test various lighting environments to create artificial circadian rhythms to improve the sleeping behaviours of patients.

\section{Strengths}

Artificial lighting allows environment to mimic the circadian rhythms of patients, allowing variation to cater for specific individuals and their unique sleeping behaviour.

Research lab located in at Massey University campus, thereby strengthening its involvement and exposure within the pre-existing medical community

\section{Weaknesses}

The compact size of the research facility means there is a lack of mobility for patients during their stay.

The interior of the facility is inappropriate for those affected by dementia, with a sterile and artificial aesthetic that lacks a stimulating and domestic quality. The lack of outdoor settings may cause feelings of isolation and imprisonment for patients during their stay.

The research lab is limited to change and flexibility, reducing its ability to change according to specific user groups or cultural backgrounds, as well as adapting to new research practices and technology improvements in the future
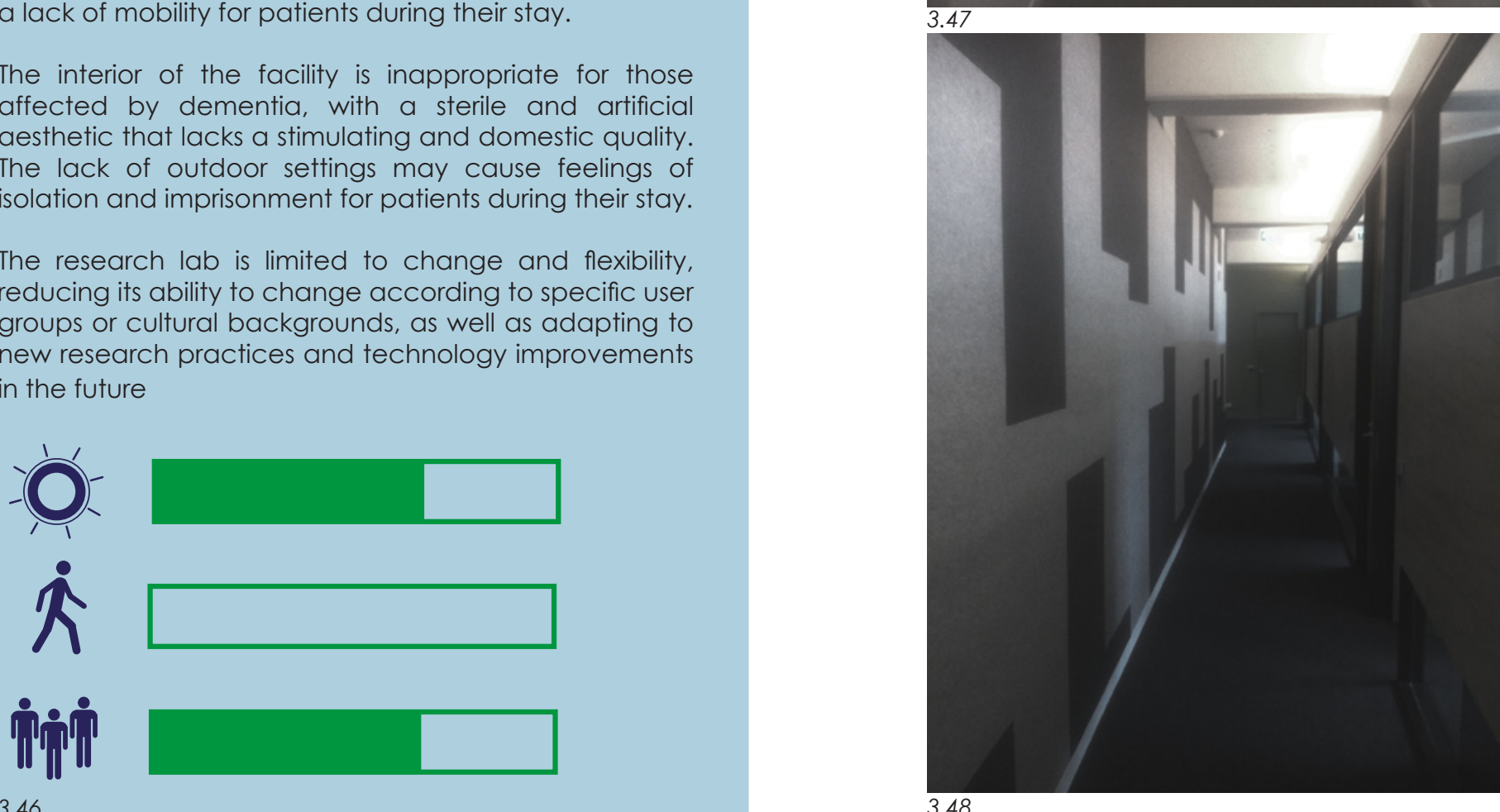

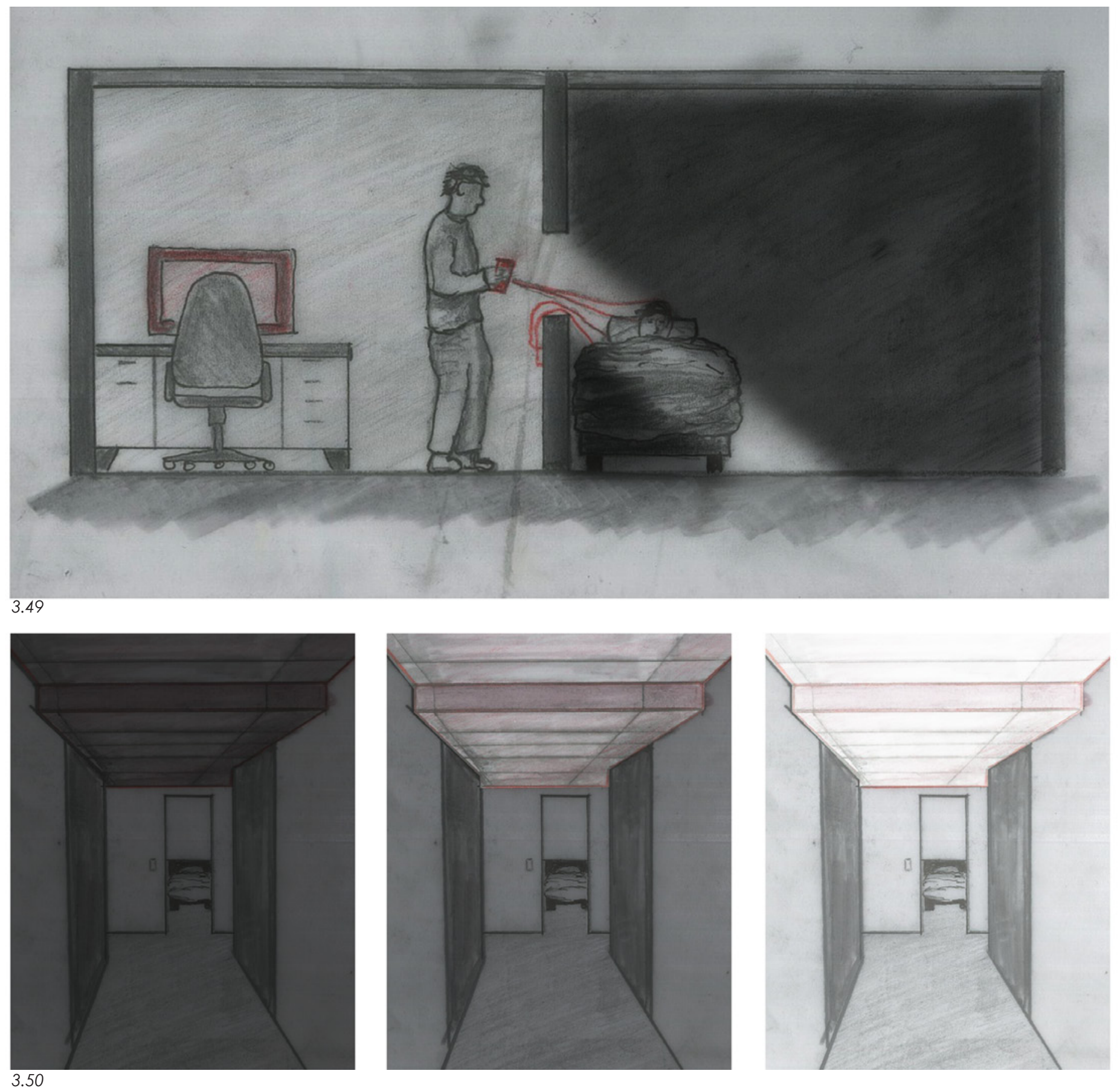

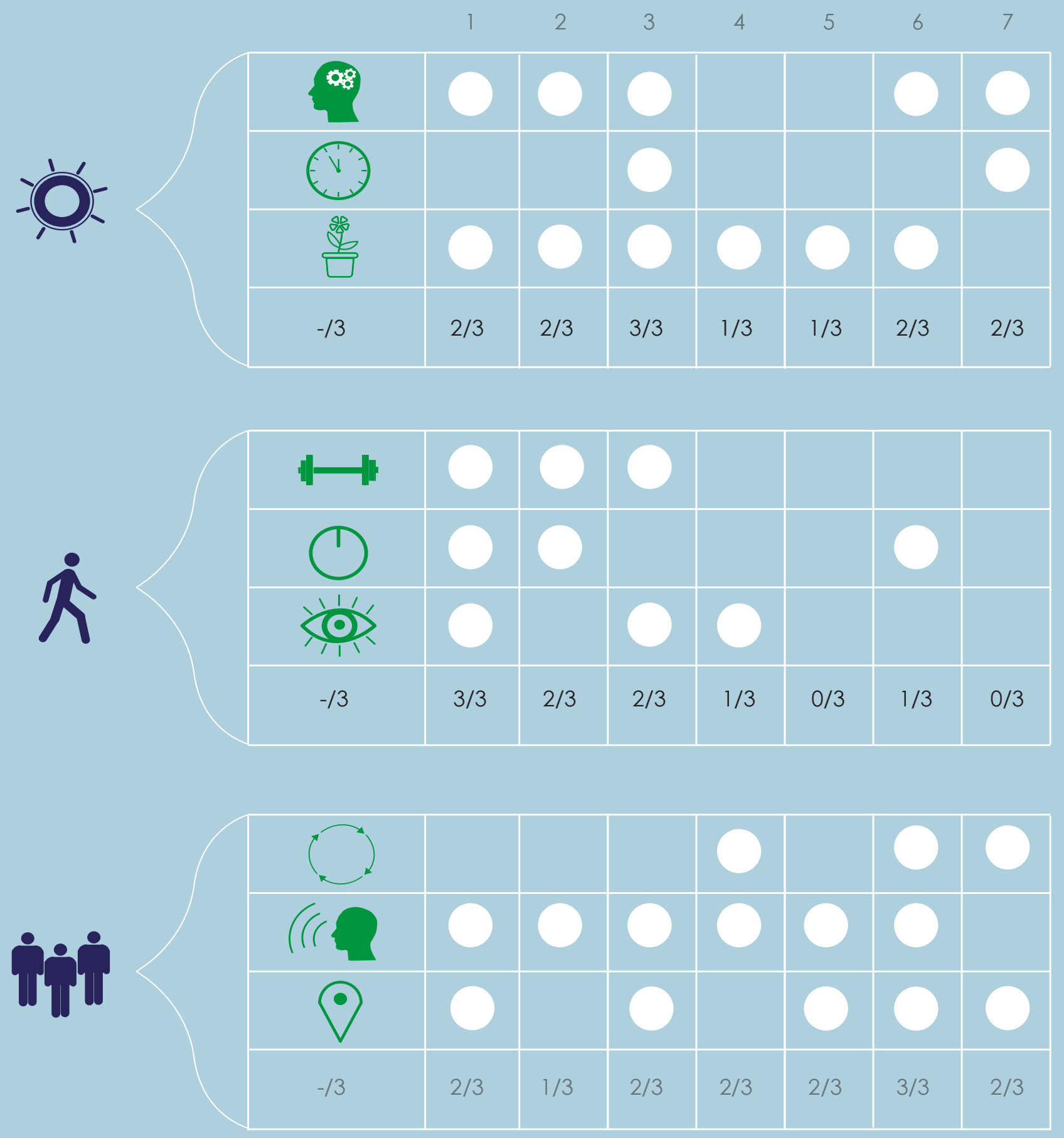

3.51 Summary of results 


\section{Summary + Reflection}

The analysis of precedents identified key design methods to implement and avoid towards achieving the desired design criteria for sleep therapy. Figure 3.51 summarizes the performance of each precedent against these architectural strategies, identifying areas of inherent strength and weaknesses towards achieving the overall design objectives:

Accessibility to outdoor spaces for multisensory stimulation and activities was considered strong, although use of light to improve circadian rhythms was limited by predominance of artificial light.

While many designs implemented diverse material and colour palettes to improve orientation for residents, building layouts and programmatic arrangement reduced effectiveness of wandering behaviour as therapeutic tool for exercise

The use of communal spaces for shared activity amongst residents was popular, although integration of dementia care facilities with external communities was severely lacking.
1. De Hogeweyk
2. The Five Gardens
3. Alzheimers Respite Centre
4. Village at the Park
5. Te Hopai
6. Sprott House
7. Massey University Sleep/Wake Research Centre 


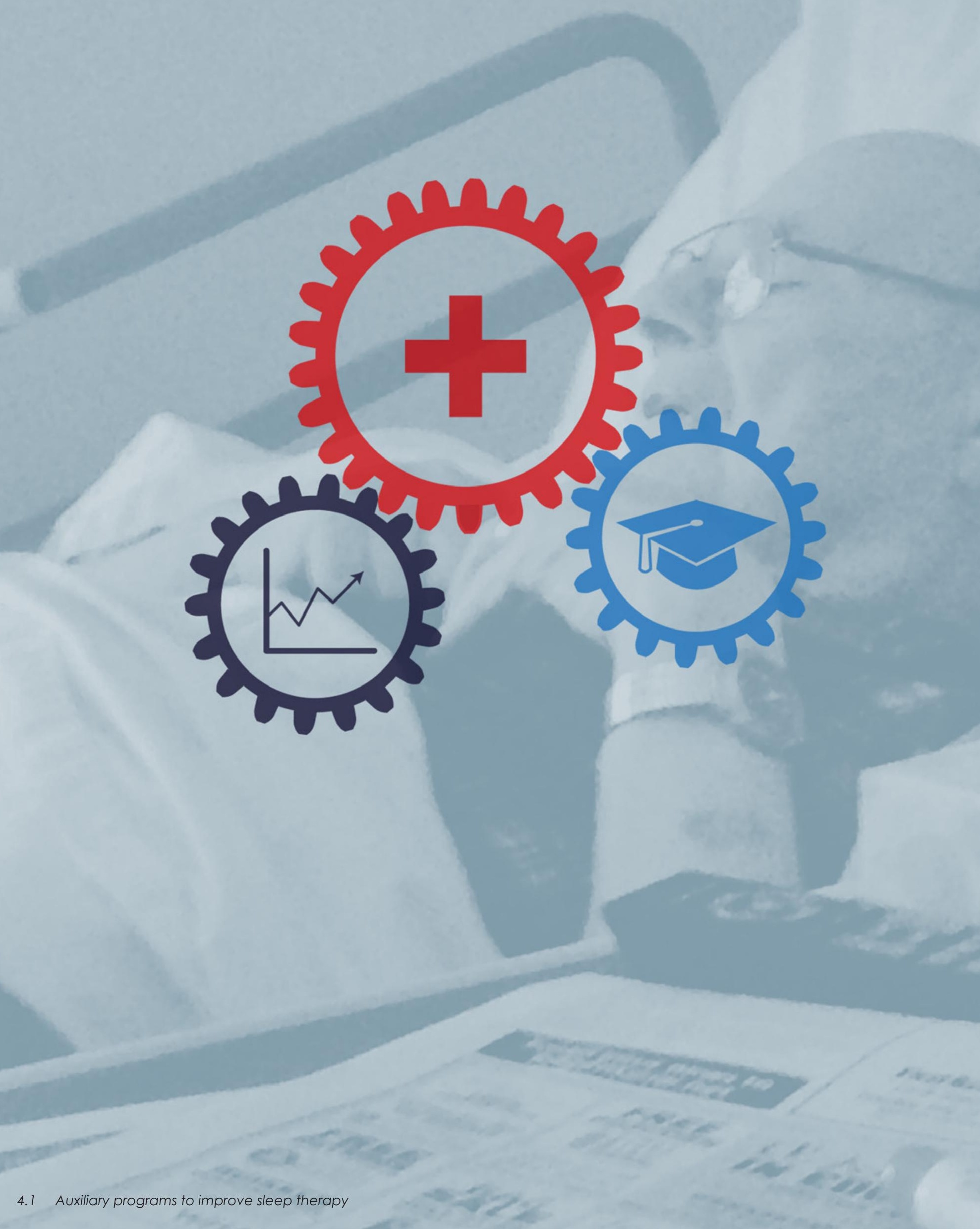


4

\section{Program Analysis}

The following chapter develops the programmatic requirements of New Zealand's first sleep therapy and research facility for dementia. 


\section{Spectrum of Care}

As the relationship to the environment and sleep remains a relatively new area of research within dementia care, the integration of auxiliary programs for dedicated research and educational spaces aims to attract people working within the medical community to further develop this new area of dementia care. Integrating the wider community within dementia respite facilities was identified in the literature review as a method to reduce the sense of isolation experienced within care facilities. The appeal of research and education spaces orientated to the medical community proved an appropriate response towards establishing mutual benefits for both groups utilizing the same facility (Figure 4.2). The integration of these auxiliary programs aims to improve knowledge and treatment for sleep in order to slow the progression of mild to moderate symptoms of dementia. By targeting the most problematic symptoms early, sleep therapy and research aims to provide treatment alongside home care to reduce the overall time spent in full time institutional environments (Figure 4.3).

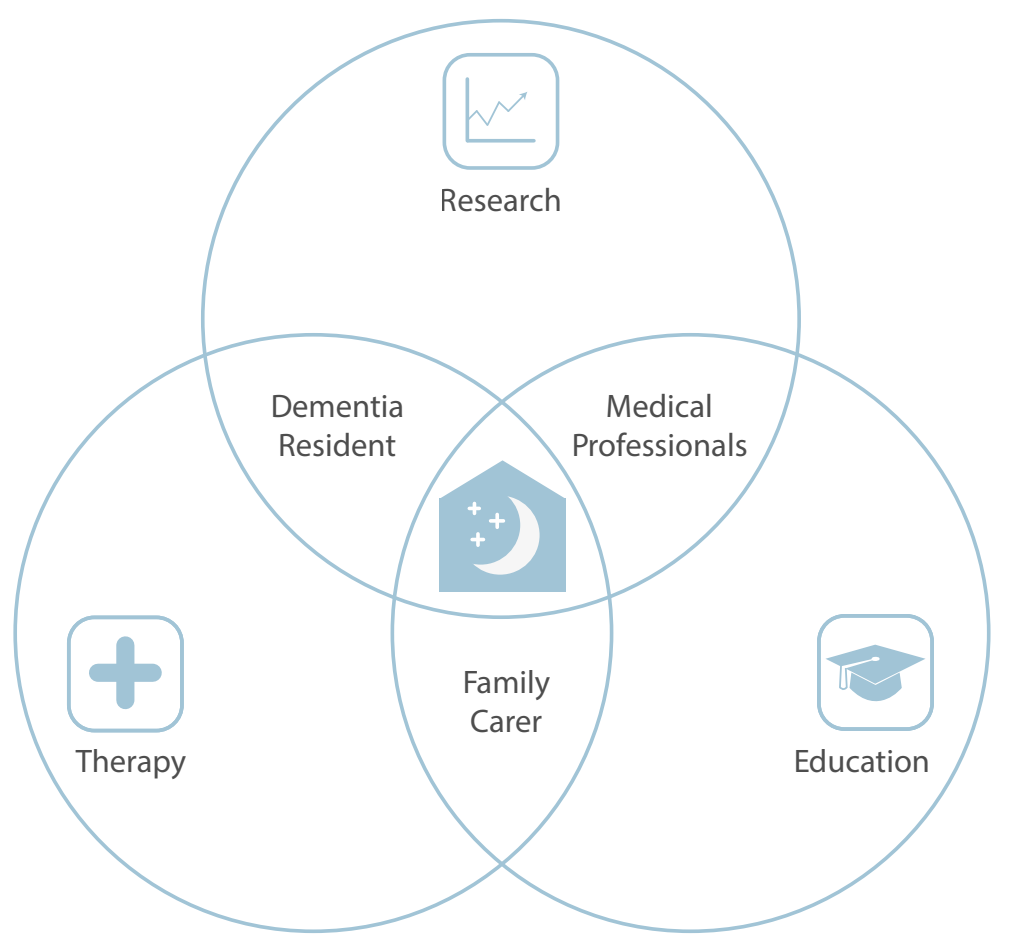

4.2

\footnotetext{
4.2 Programs and primary users

4.3 Integration of facility within spectrum of care
} 

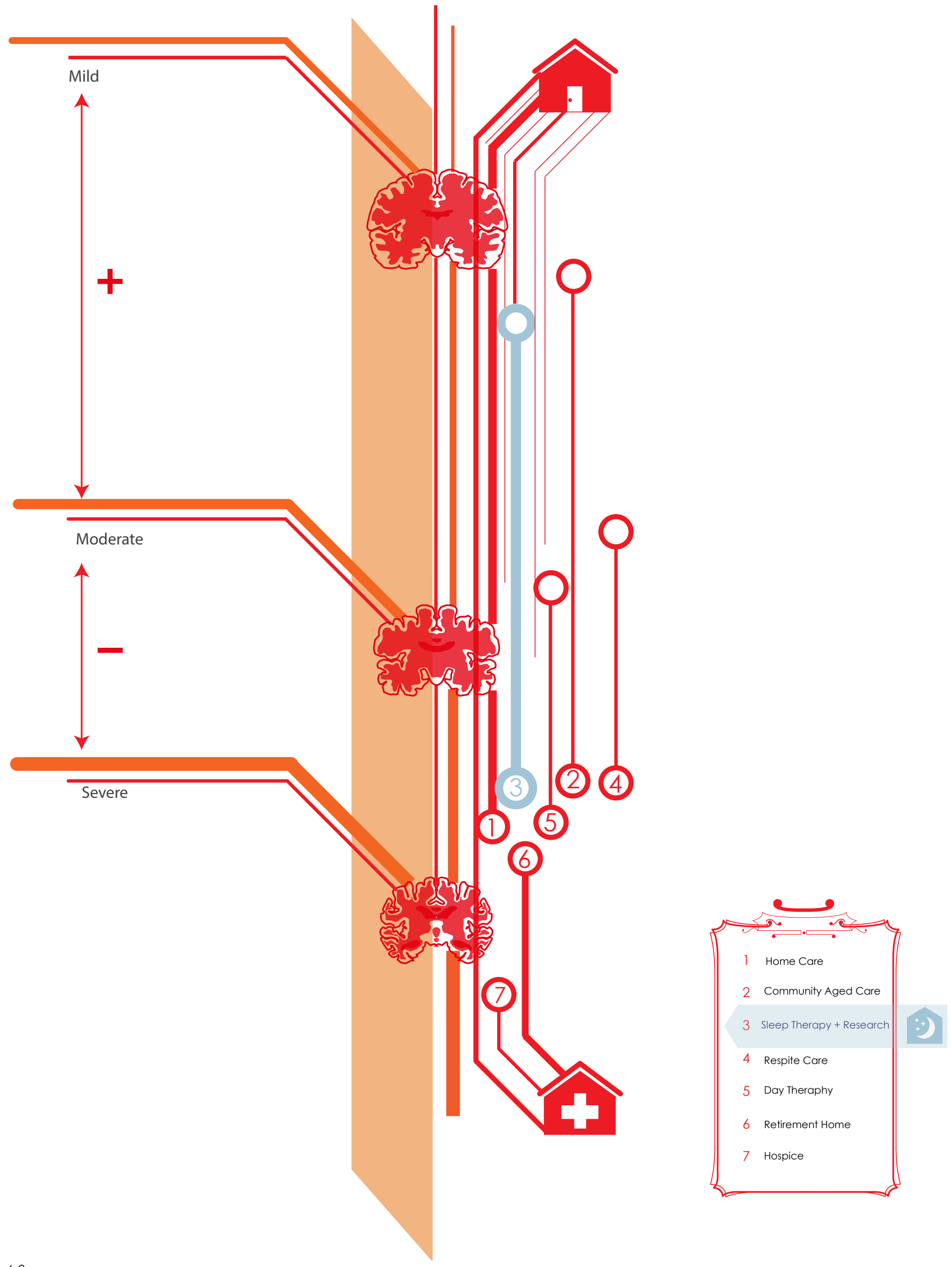


\section{Program Allocation + Area Schedule}

The programmatic requirements in establishing a sleep therapy and research facility were refined through area schedules that were generated through use of the metric handbook, case study analysis and medical research (Figure 4.4).

Key programmatic findings included:

Restricting respite unit to 10 bedrooms as "larger facilities increase agitation and are confusing and high quality care is easier to provide in small groups" (Fleming et al 3)

Short-term accommodation units for family carers, staff and other medical professionals that will contribute to sense of community for residents and increase the research capacity of the facility by accommodating these groups living outside the immediate area

Library, lecture theatres and teaching rooms that are orientated to medical community provide opportunities for improving dementia education alongside community interaction for residents

Small research and accommodation unit aligns with person-centered approach to care that is adopted in local care facilities. 
Respite Accomodation

Area $\left(\mathrm{m}^{2}\right)$

$10 \times$ Bedroom + Ensuite 200

Kitchen + Dining 80

Living Room (Morning) 25

Living Room (Day) 24

Living Room (Evening) 24

Outdoor Garden 300

Contemplation Room $\quad 15$

Staff Room 40

Activity Room 36

Counciling Room $\quad 10$

Gym $\quad 30$

2x Bathroom 16

Laundry 10

Family + Staff Accomodation

Area $\left(\mathrm{m}^{2}\right)$

$15 \times$ Single Bedroom Flats $\quad 420$

Gym

30

Laundry

Library

Area $\left(m^{2}\right)$

Cafe

200

$2 \times$ Tutorial Room

80

Seminar Room

40

Lecture Thetare

Toilets

50

230

16

Dementia Research

$8 \times$ Office

Staff Room

Meeting Room

Technical Lab

Toilets

Storage
Dementia Research Accomodation Area $\left(m^{2}\right)$

Bedroom + Ensuite

20

Kitchen + Living

Garden Space
20

50 


\section{Daylight Hierarchy}

Establishing a hierarchy was important towards refining programmatic arrangement on site in order to maximize natural light efficiency of the building (Figure 4.5). Key areas within the hierarchy identified the following:

The need for morning light stimulation placed respite bedrooms at the top of the hierarchy in order to achieve effective sleep therapy

Living, kitchen and outdoor spaces also required maximum light exposure within areas of intended high use amongst residents

Natural light exposure for short-term accommodation units was important to provide a comfortable living environment for family caregivers, staff and other medical professionals who also lived on site

Providing well-lit areas to maintain cognitive levels for learning and attention within teaching areas.

Sleep research accommodation unit that follows similar design principles to the Sleep/Wake Research Centre was lowest in hierarchy due to its use of artificial lighting rather than natural light for therapy 


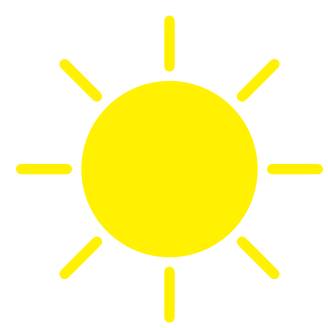

$10 \times$ Bedroom + Ensuite

Kitchen + Dining

Living Room (Morning)

Living Room (Day)

Outdoor Garden

Contemplation Room

5 x Single Bedroom Flats

Library

2x Tutorial Room

Seminar Room

8x Office

Cafe

Staff Room

Staff Room

Activity Room

Counciling Room

Gym

Meeting Room

Technical Lab

Gym

Living Room (Evening)

Lecture-Thetare

Toiltes

2x Bathroom

Laundry

Toilets

Storage

Laundry

Bedroom + Ensuite

Kitchen + Living

Garden 


\section{5}

Site Analysis 


\section{Establishing a Community}

New Zealand's first dementia research facility was opened in 2014 at the University of Auckland's Centre For Brain Research (University of Auckland). The facility is located in the Auckland hospital precinct, strengthening its appeal within a preexiting medical community. As the focus of environmental therapy will be adopted in this new dementia research facility, locating the testing site in the capital city of Wellington will provide additional exposure to institutes such as the Ministry of Health to help promote awareness to medical communities throughout New Zealand (Figure 5.2).

Mapping the pre-existing sites of dementia care and medical research facilities helped to identify areas of interaction between these pre-existing communities (Figure 5.3). Newtown was identified as an appropriate site for a dementia sleep therapy and research facility with close proximity between these groups strengthening the appeal for community integration.

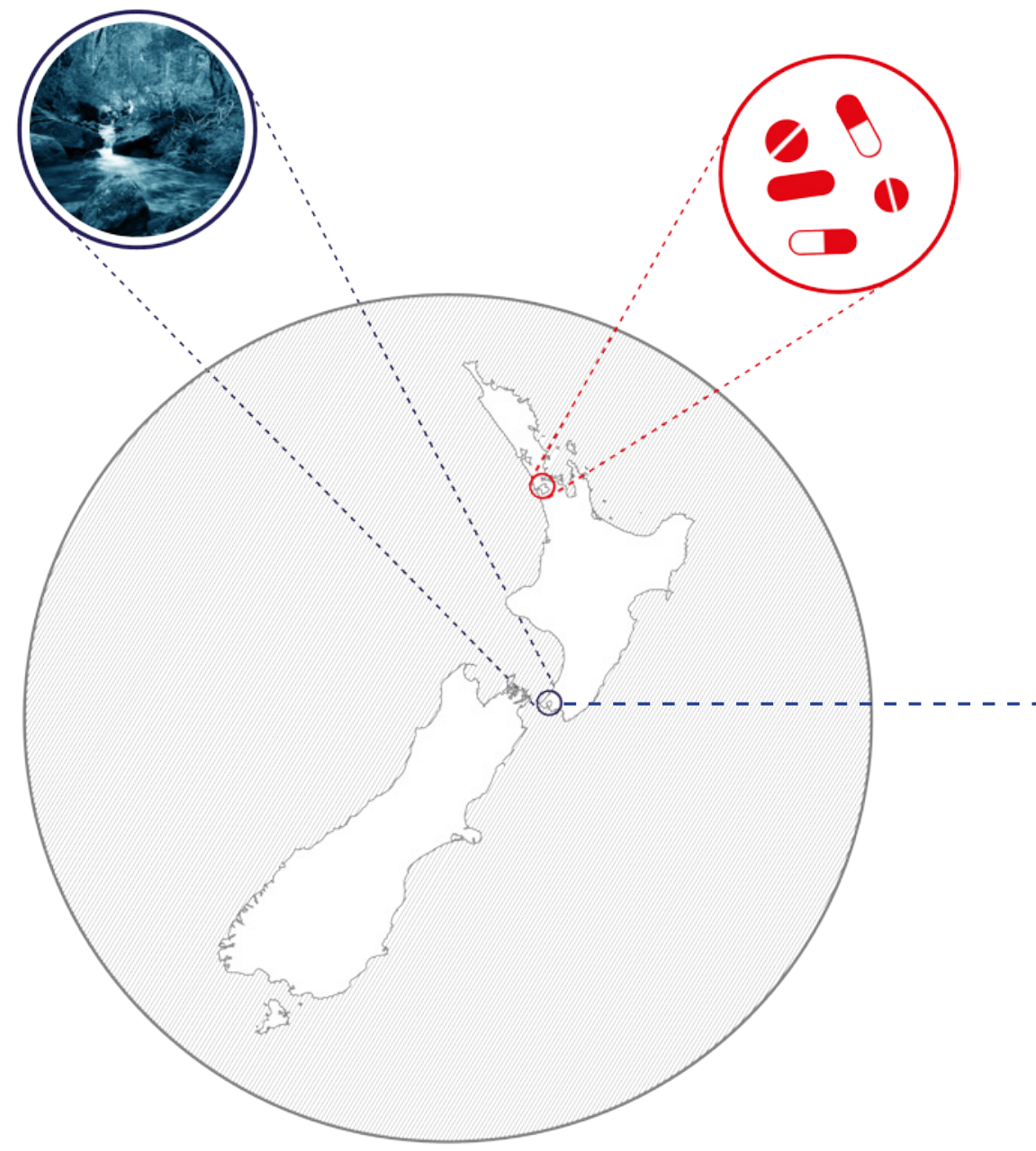

5.2 


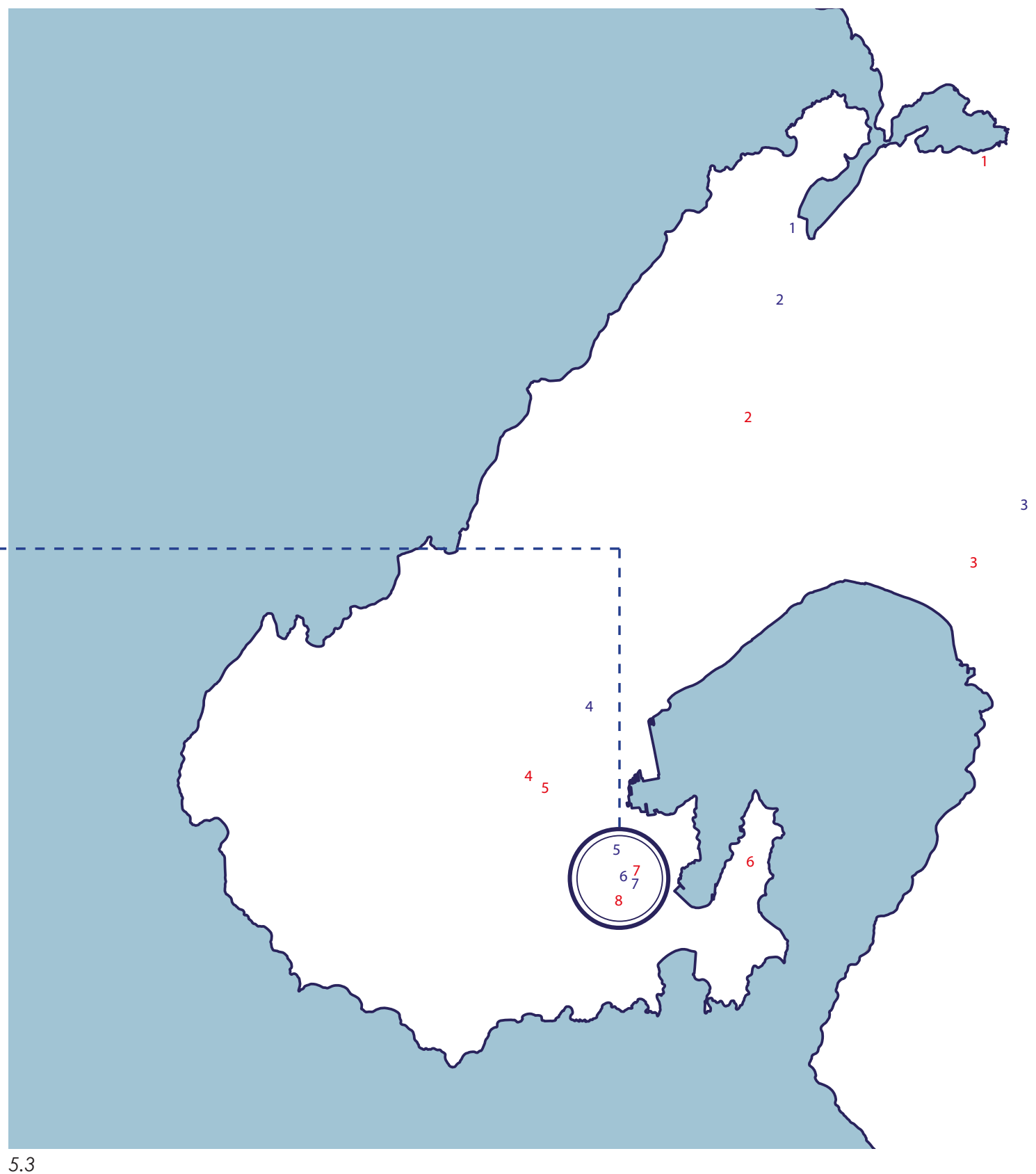

Dementia Care Facilities

Whitby Rest Home and Hospital Redwood Club

Ferguson Rest Home and Hospital Marsden DayCare Trust

Sprott House

Millvale House Mirimar

Te Hopai

Village at the Park
Medical Research Facilities

1 Whitireia Community Polytechnic 2 Kenepuru Hospital

$3 \quad$ Hutt Hospital

4 Bowen Hospital

5 Massey University

6 Victoria University

7 University of Otago 


\section{The Site}

The close proximity to Wellington Hospital, Massey University and bus routes to Wellington city make it an appealing an accessible location for the medical community (Figure 5.5). Similarly, close proximity to the Wellington town belt offers residents opportunities to be exposed to natural elements on site. The potential for development on the exiting car park and vacant Riddiford building highlights an opportunity to reuse the space that minimizes impact towards the medical precinct (Figure 5.4).

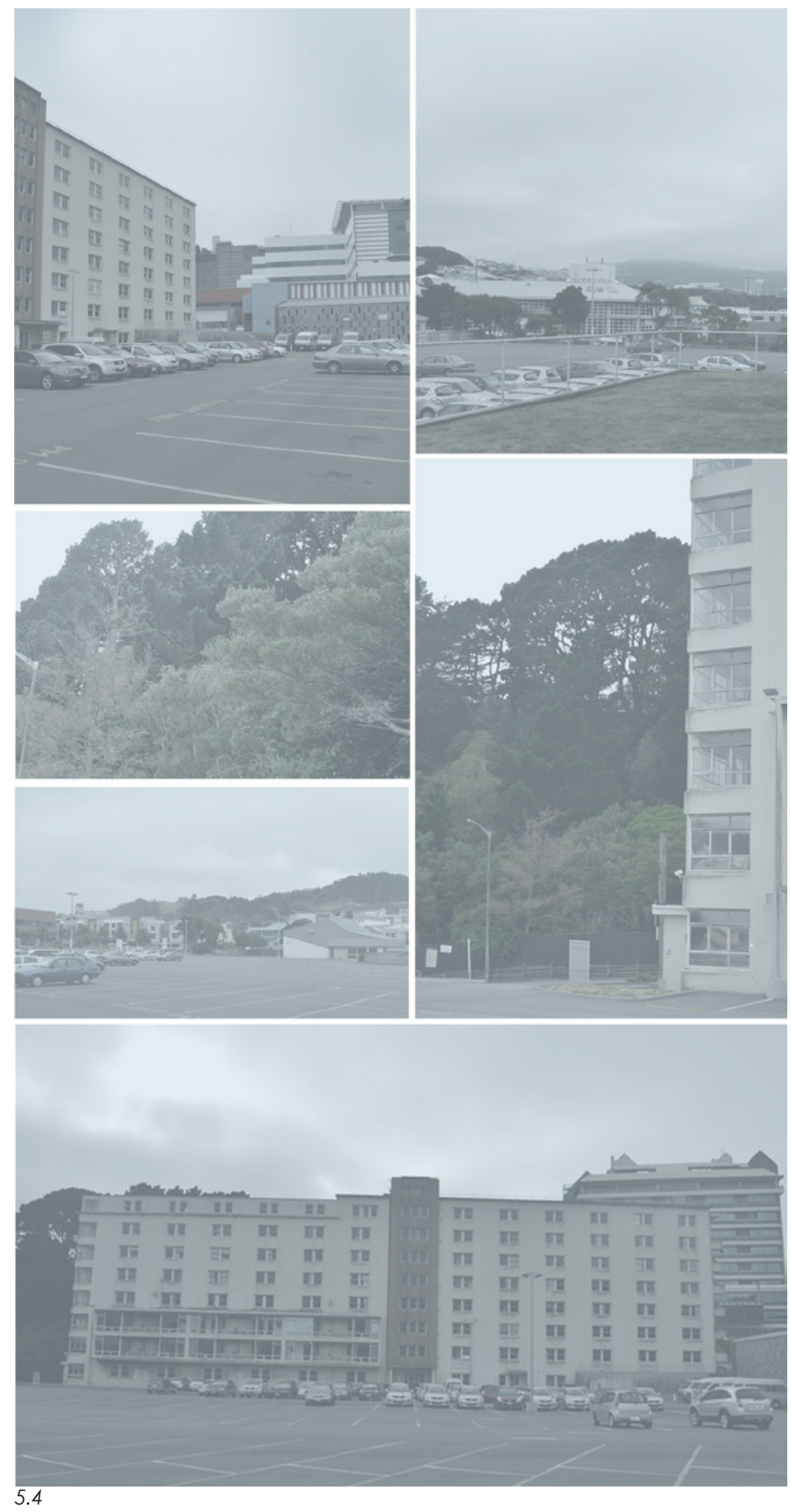




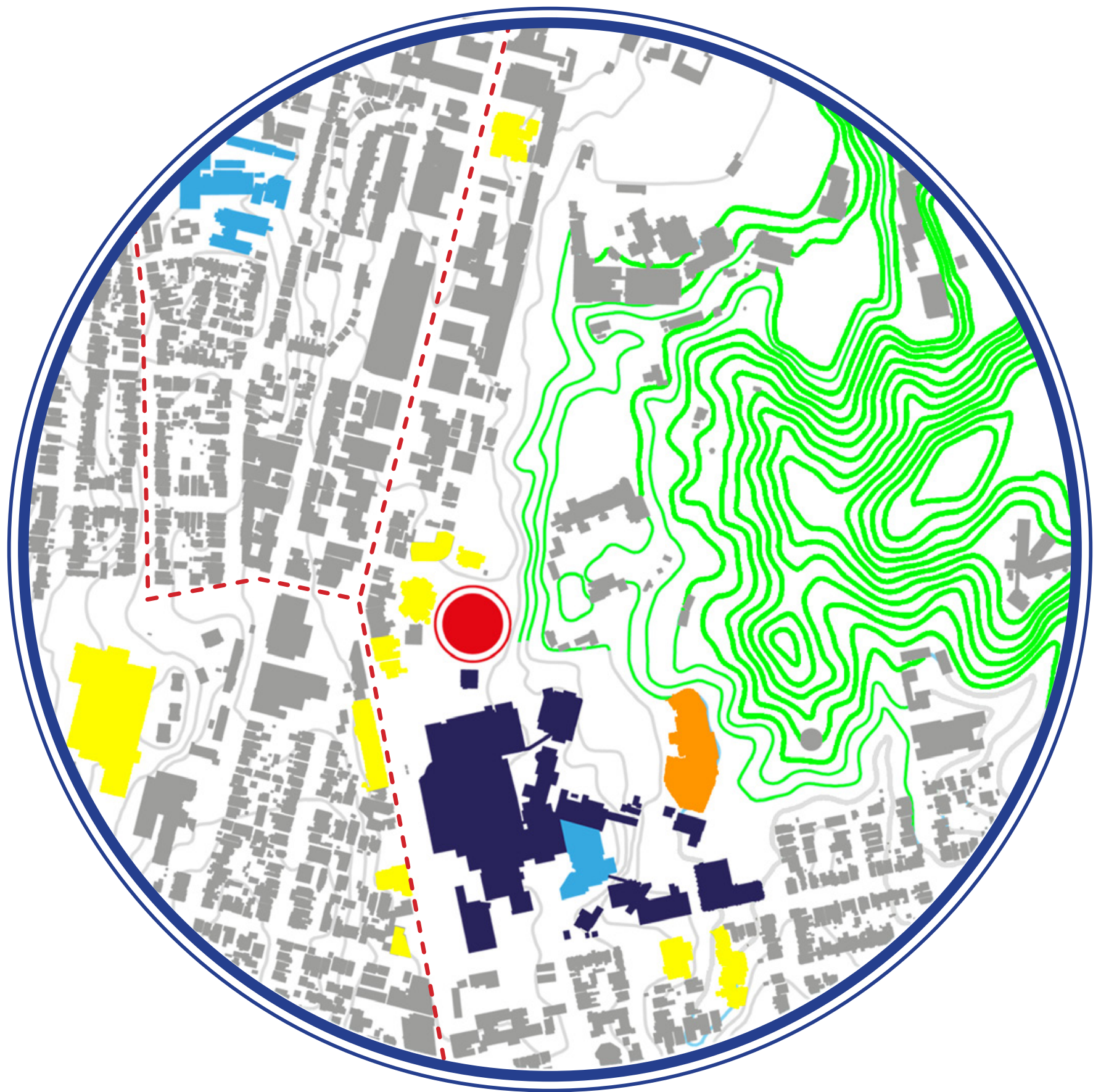

$\downarrow \square \square \longdiv { 1 0 0 \quad 2 0 0 m }$

- Site

- Dementia Unit

Other Medical Facility

- Medical Research Facility

- Wellington Hospital

- Town Belt

- Main Bus Routes 


\section{Static vs Dynamic}

This dichotomy between static built forms in relation to dynamic natural elements was explored through a series of photographs and drawings to express the juxtaposition of these elements that occupy the site (Figure 5.6 - 5.7). This contrast in movement provided inherent characteristics of the site that is located on the border between urban and natural elements (Figure 5.8).
5.6 Interaction between natural and built elements 5.7 Abstract representation of static and dynamic elements on site

5.8 Relationship between natural and urban space
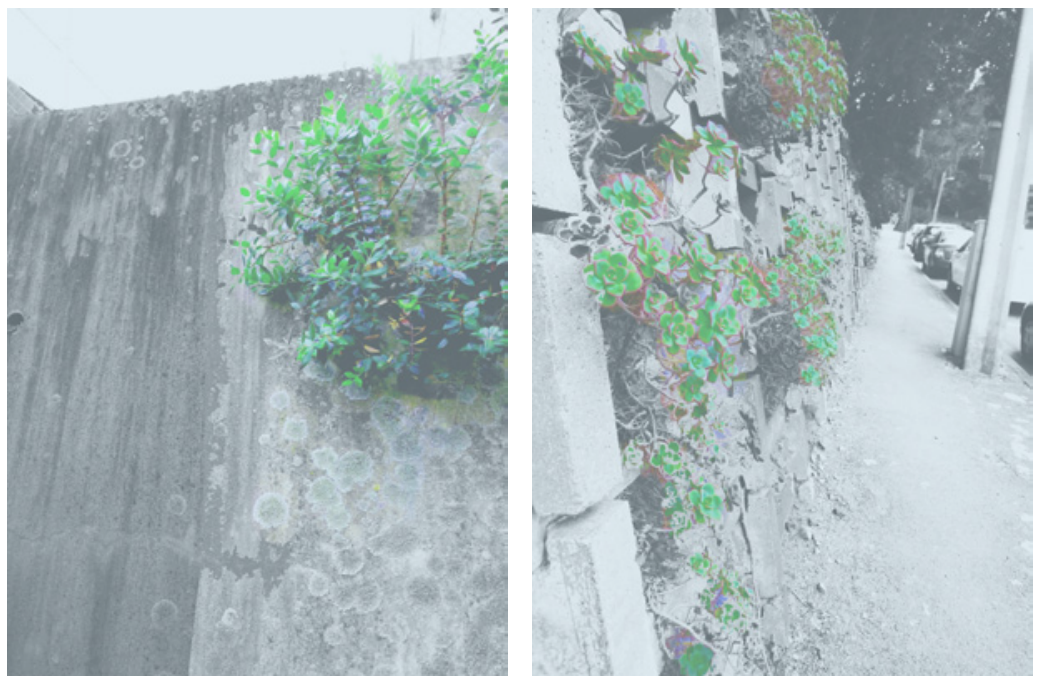

5.6
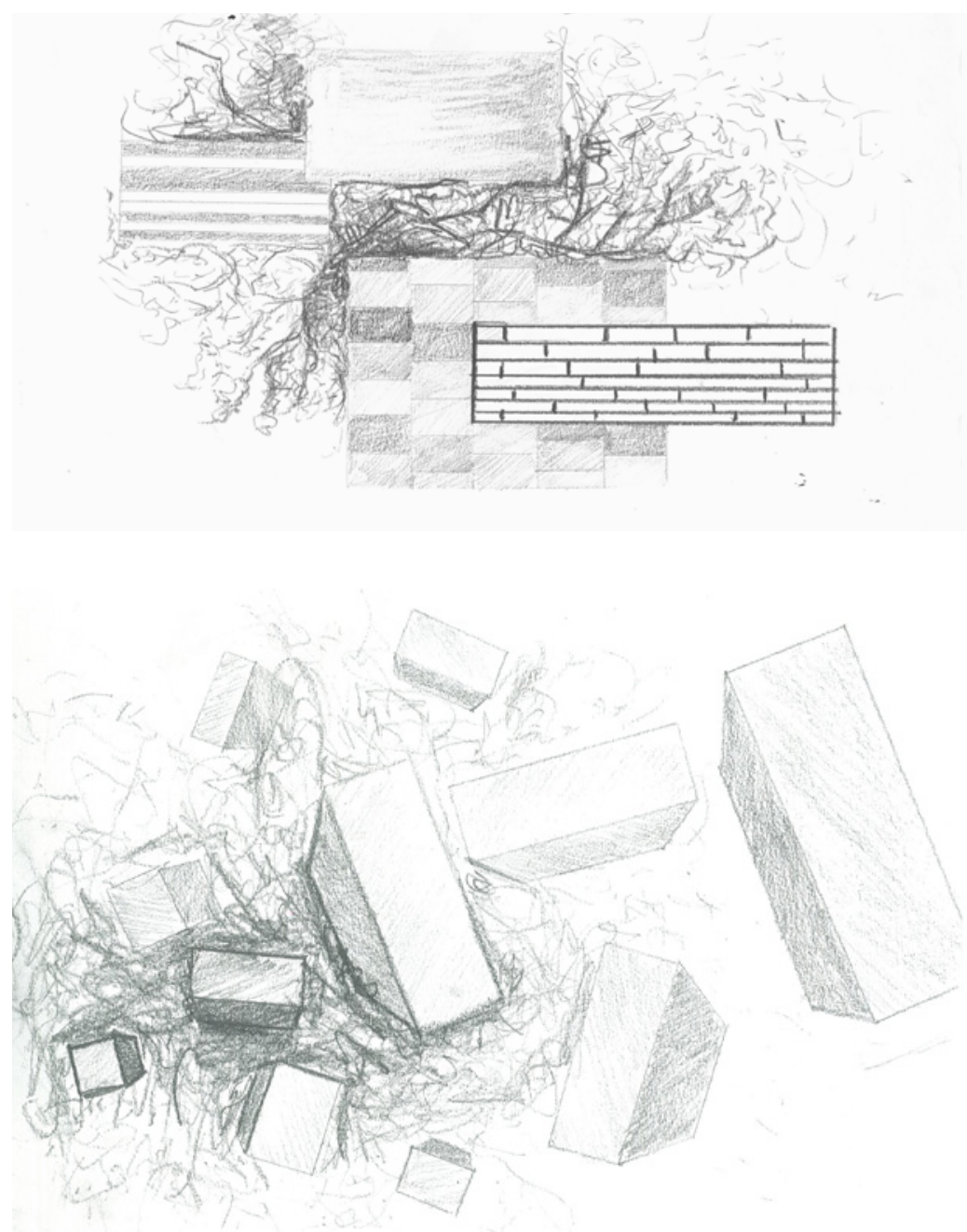

5.7 


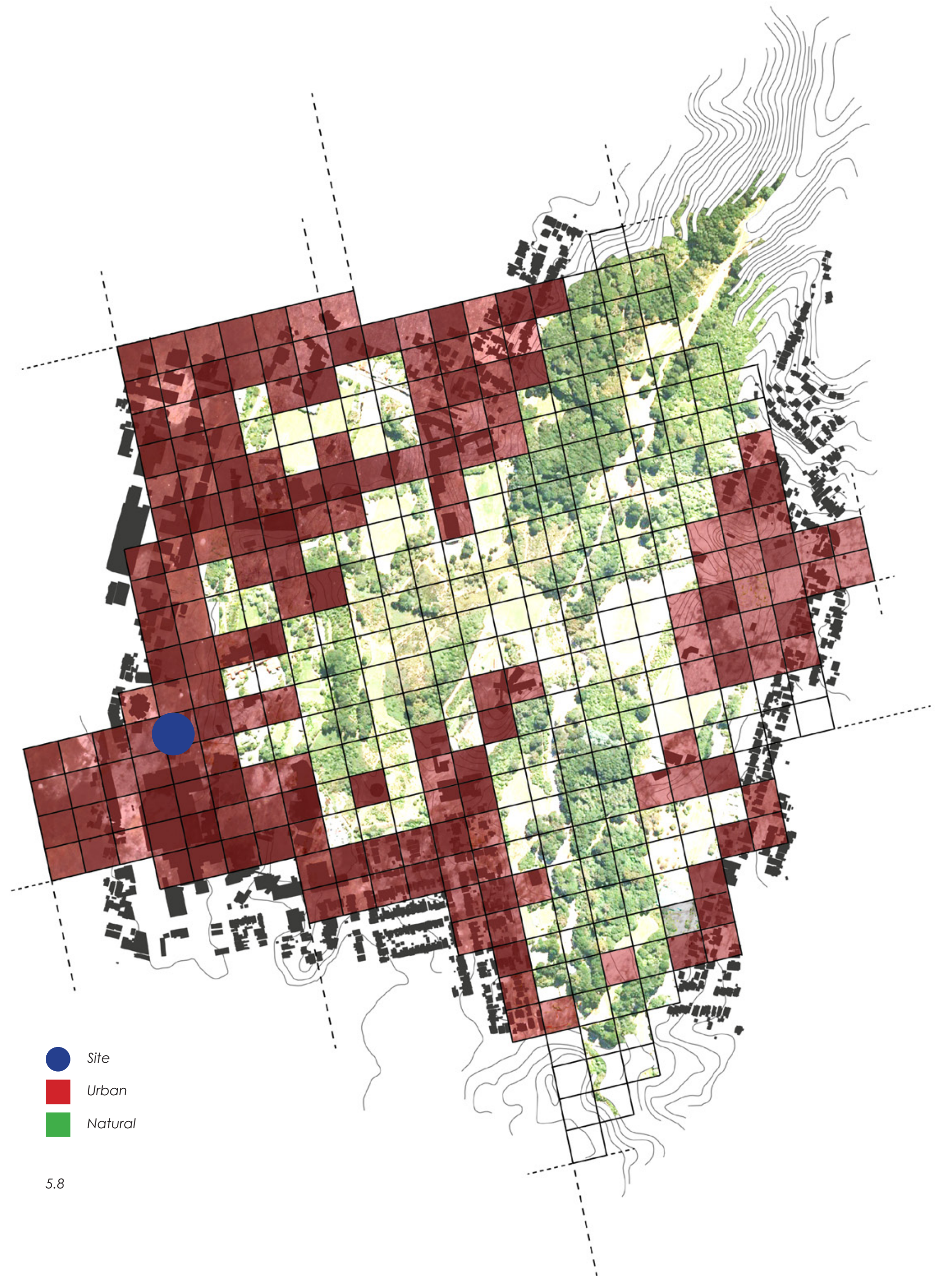




\section{Natural Light Studies}

Lighting studies measured morning and evening light during different times of the year to identify natural light patterns that were characteristic to the site (Figure 5.6). The analysis identified the steep contours of the town belt reduced efficiency of morning light on site (Figure 5.8), requiring dementia resident's bedrooms to be positioned on the western perimeter of the site to maintain efficient light exposure (Figure 5.7).
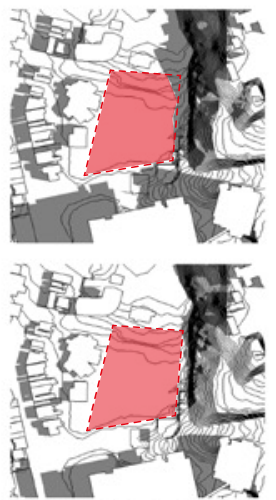

0900

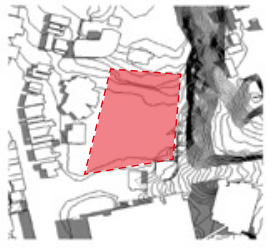

1600

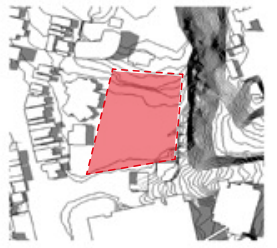

1700

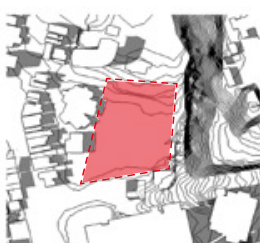

1800
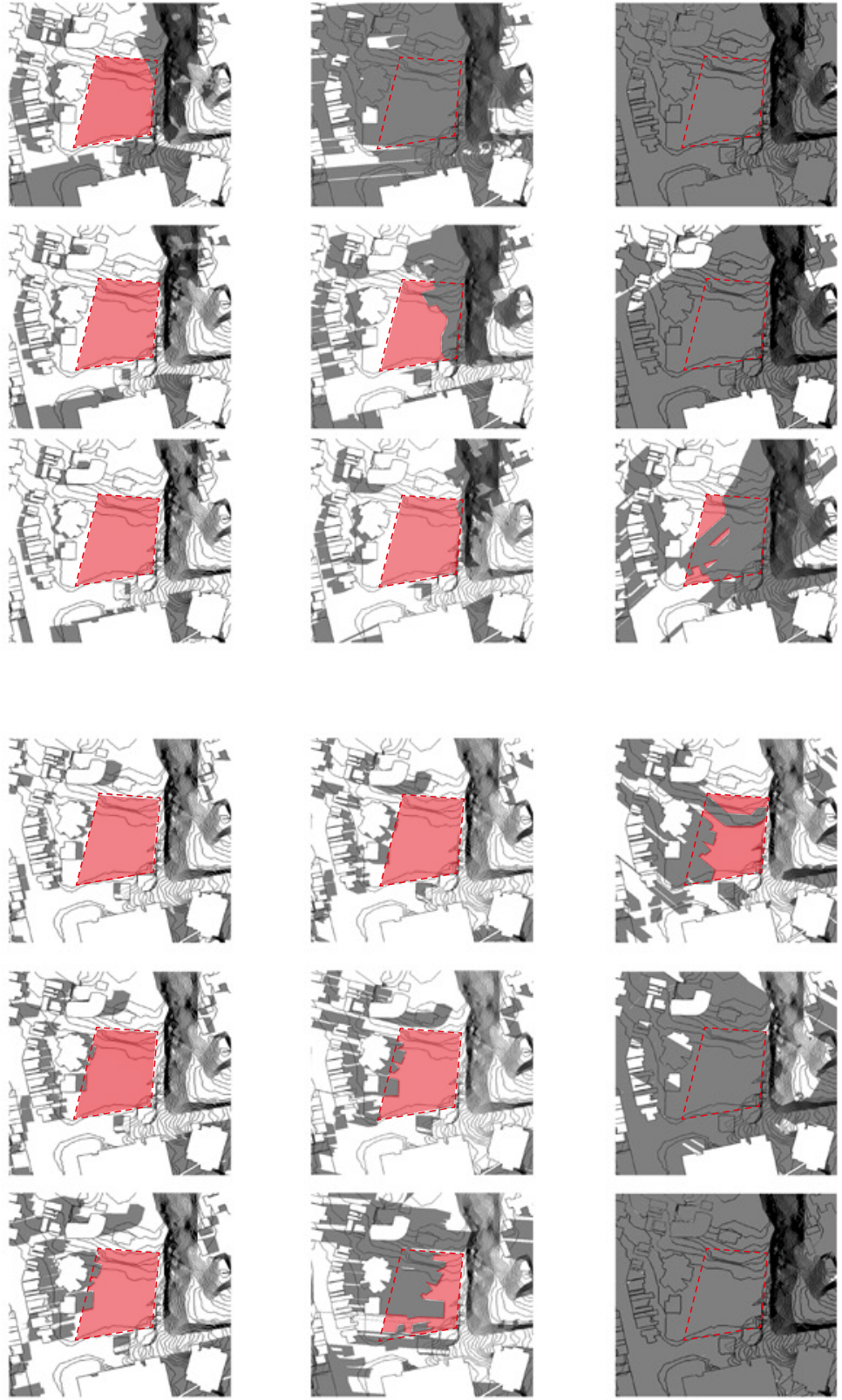

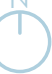

Daylight Exposure

- Site Boundary

5.6 

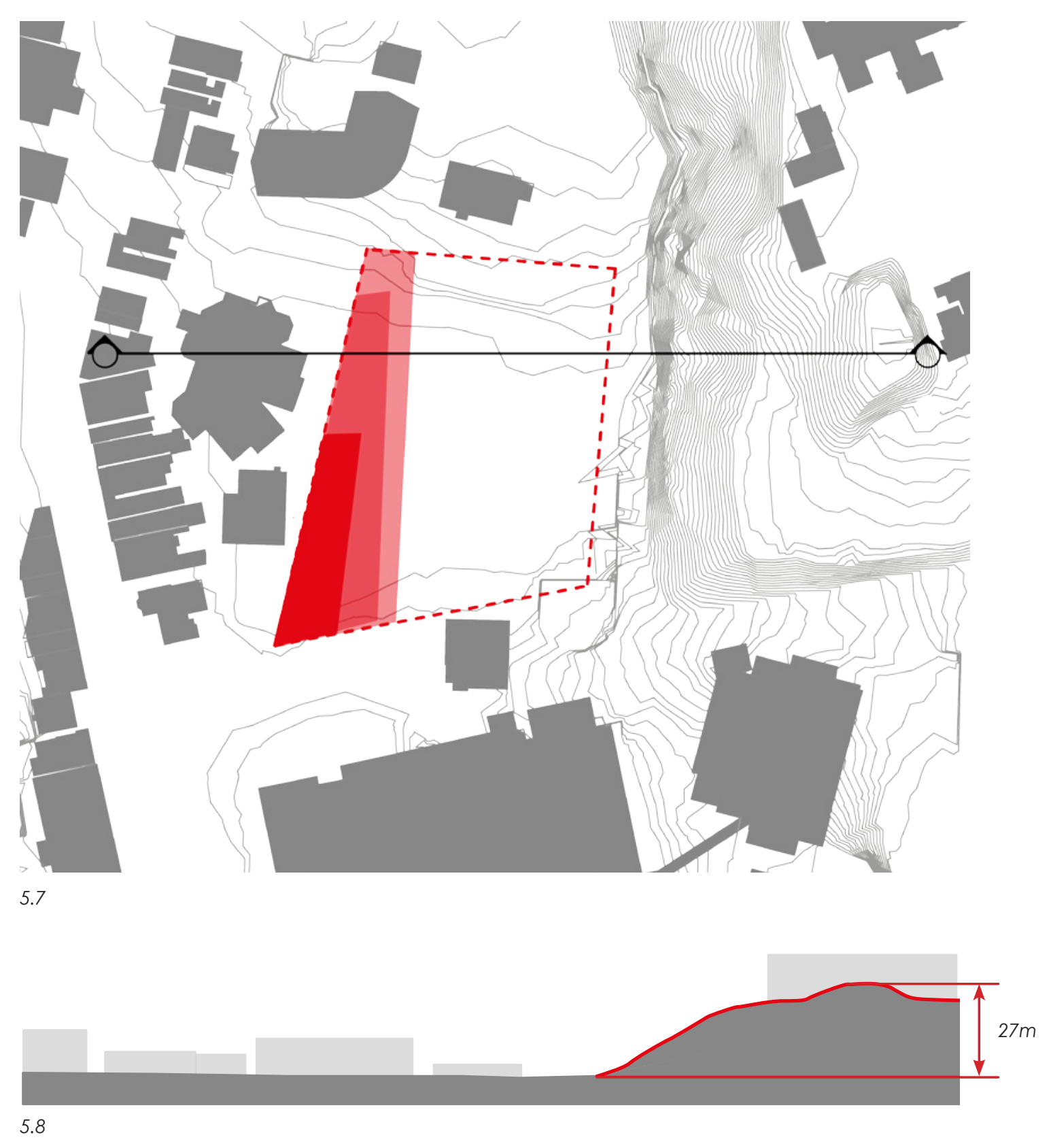


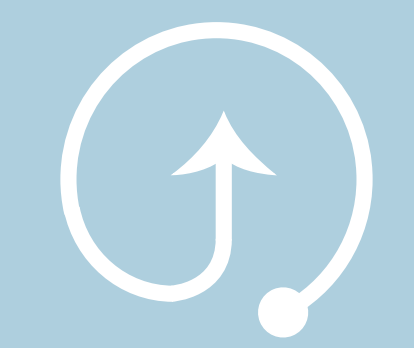

DESIGN PHASE ONE "Outside In"

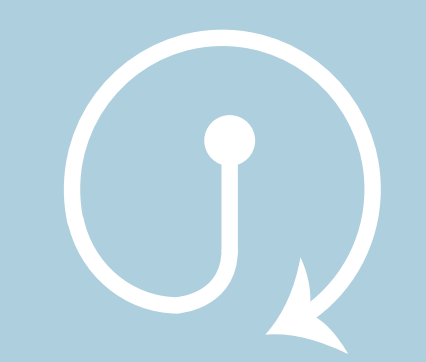

DESIGN PHASE TWO

"Inside - Out"

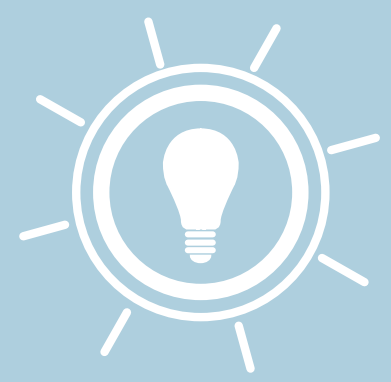

DESIGN PHASE THREE

"Simulation" 
6

Designing Sleep Therapy for Dementia 
(A) 


\title{
6.1
}

\section{Design Phase 1}

\author{
"Outside - In"
}

The following chapter explores design strategies such as architectural imagery, courtyard typologies and shared spaces that seek to attract the outside community within a dementia care environment, and in the process, foster a sense of community for dementia residents during respite. 


\section{Expressing Dementia}

An analysis of contemporary medical research and education facilities(Figure 6.1.1- 6.1.3) highlighted the use of architectural facades in communicating familial imagery of molecules, cells and biomedical forms to inform the public of the buildings function. Ideas of progression and juxtaposition that characterize dementia were expressed through a series of experimental parti models. This process developed imagery and material qualities that help convey the deterioration of the brain through architectural form (Figure 6.1.4).

6.1.1 John Curtin School of Medical Research façade detail

6.1.2 La Trobe University Institute For Molecular Science façade

6.1.3 University of Tasmania School of Medicine facade

6.1.4 Parti models expressing dementia

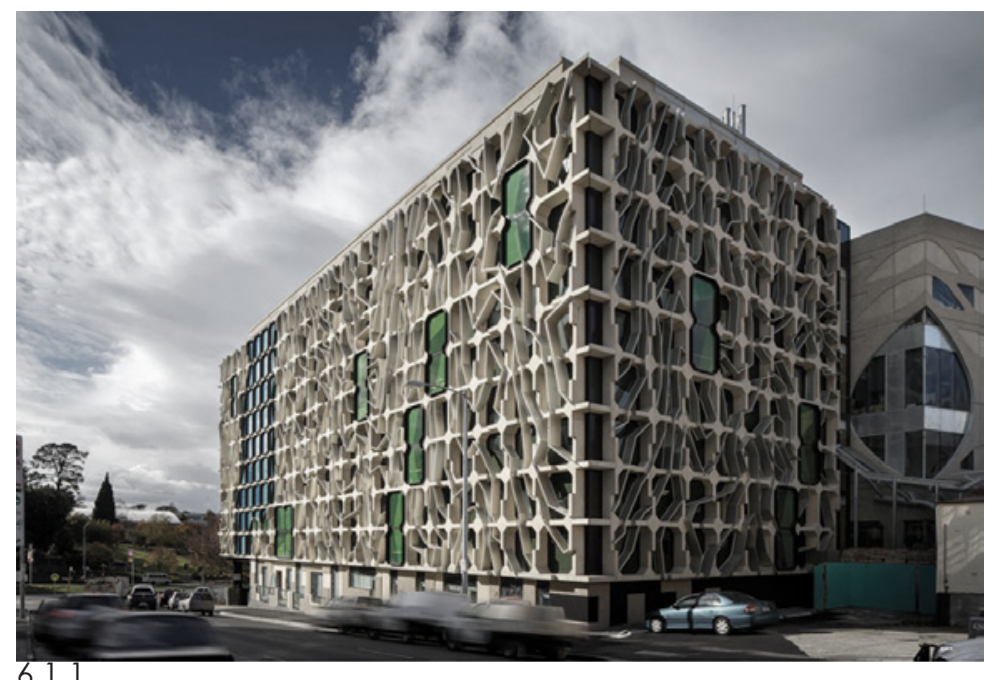

6.1 .1

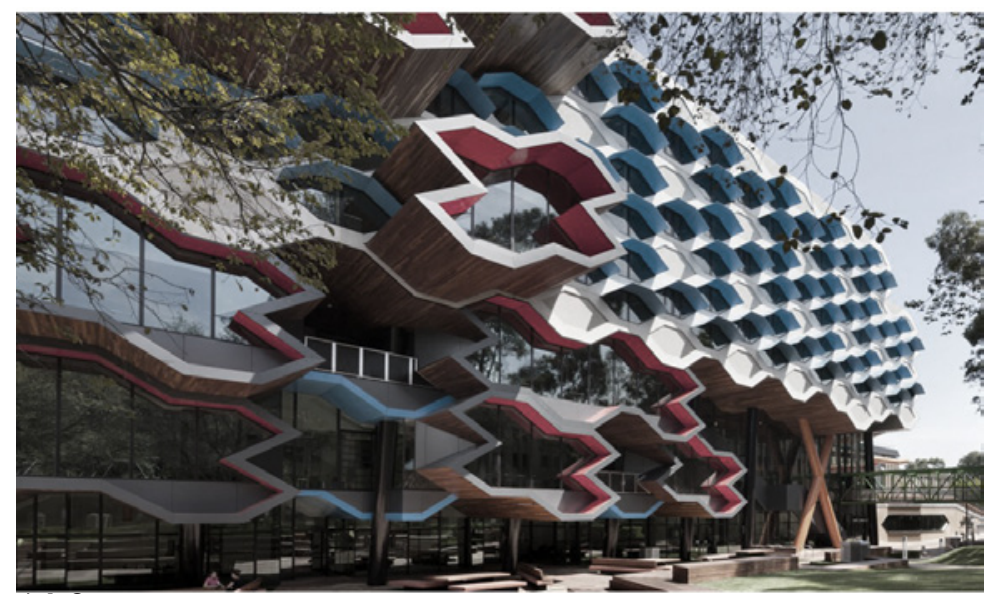

6.1 .2
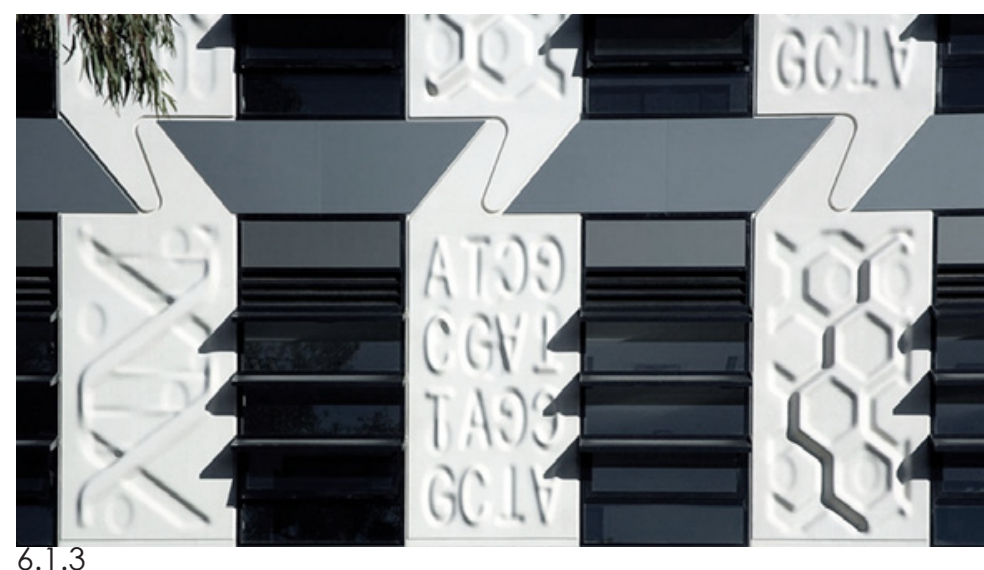

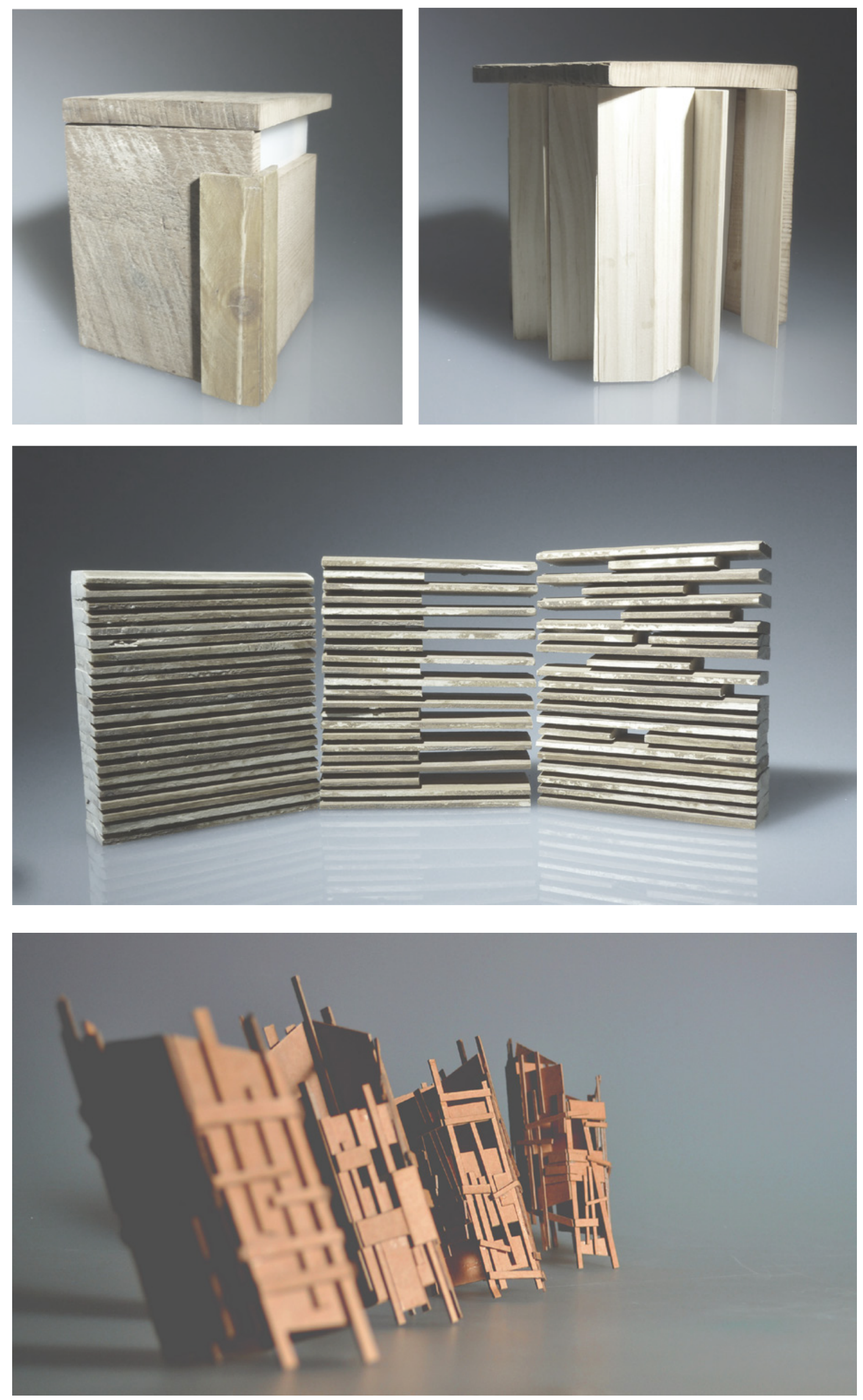


\section{The Courtyard}

The courtyard typology was explored through a series of programmatic arrangements according to courtyard form and natural light exposure on site (Figure 6.1.6). These experiments highlighted that the use of multiple courtyard forms provided an optimal balance of secure exterior spaces for residents and accessible outdoor spaces for the medical community. Further tests explored the relationship of courtyard spaces to the site by integrating green corridors that connected to the Wellington town belt (Figure 6.1.5).
6.1.5 Explorations to connect courtyard to town belt through green corridors

\subsubsection{Programmatic arrangement and courtyard type}
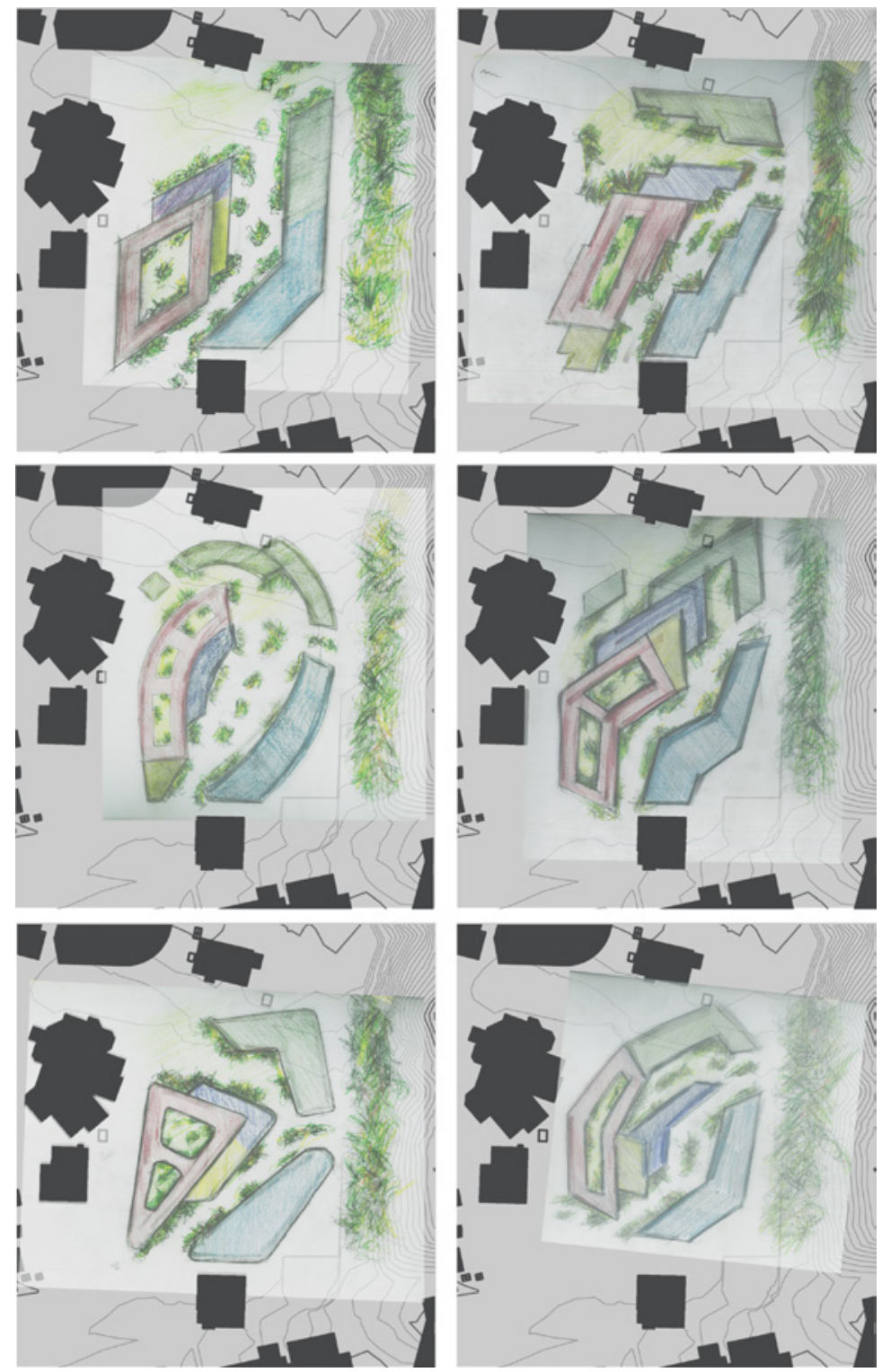

6.1 .5 
Multiple
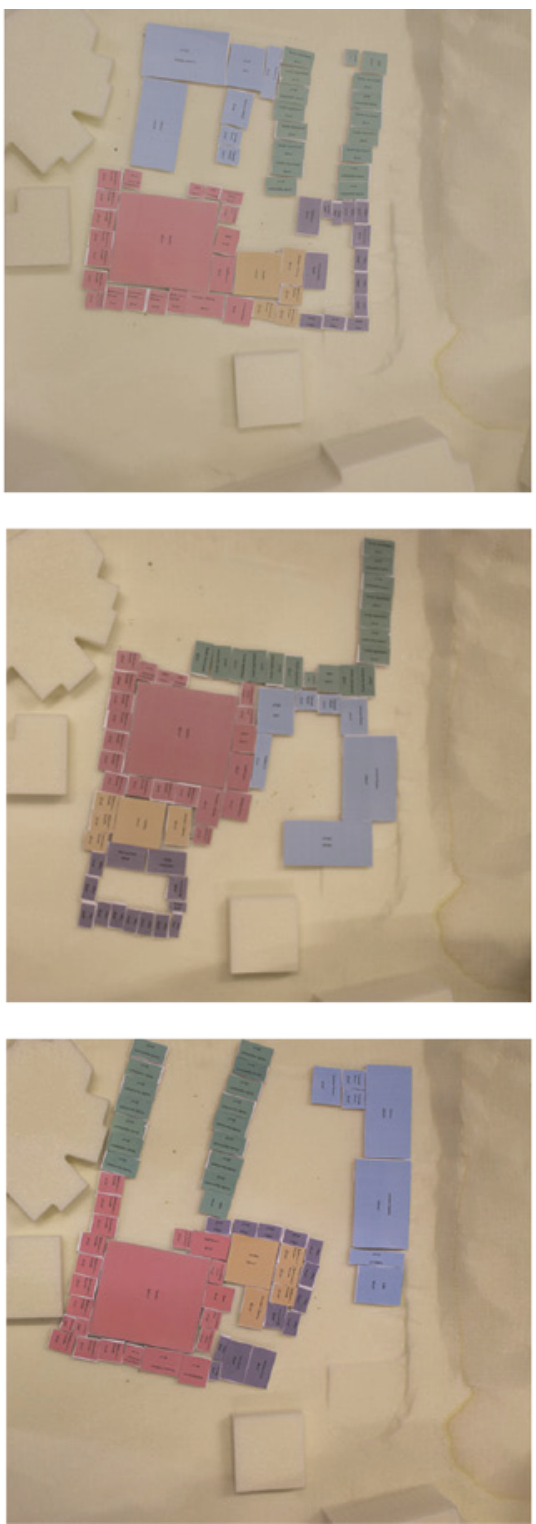

6.1 .6
Perimeter
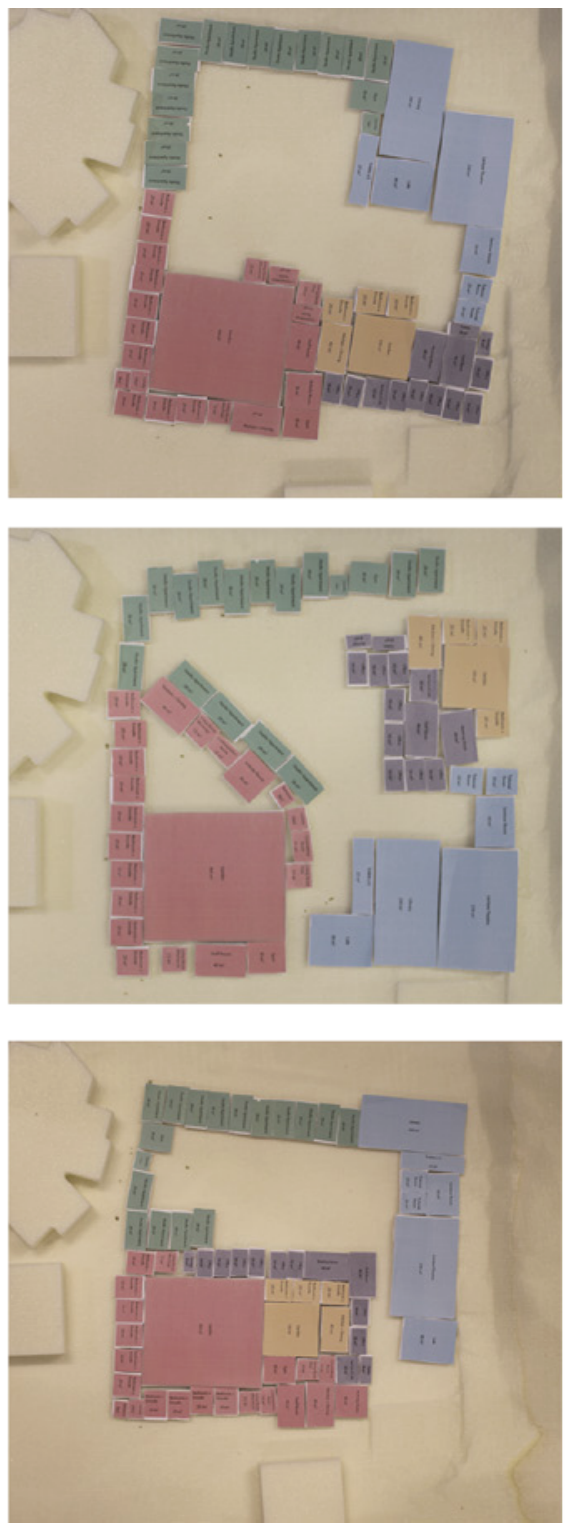

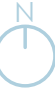

Open
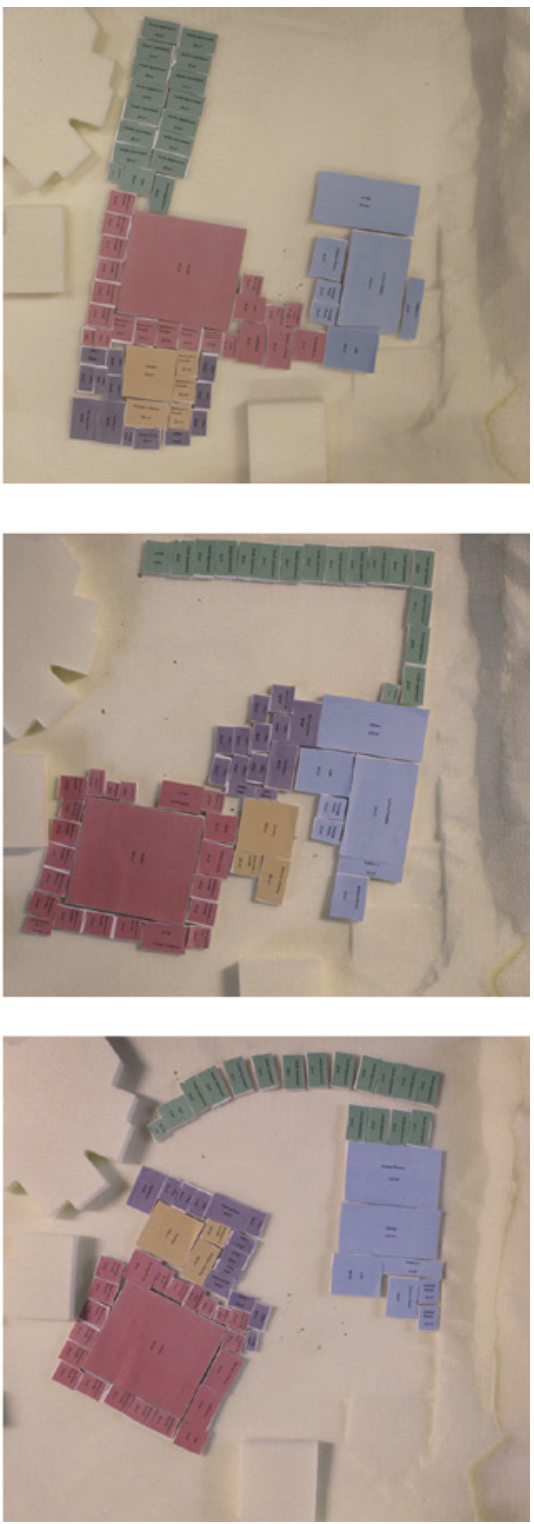

Dementia Respite Accommodation

Dementia Research Accommodation

Family + Staff Accommodation

Medical Education facilities

Dementia Research facilities 


\section{Courtyard Development}

These series of explorations tested methods to promote outdoor circulation and interaction with courtyard spaces for the medical community. Variations of canopies were tested to promote outdoor circulation (Figure 6.1.7), outdoor walkways were also explored to make visitors feel actively engaged with the external environment as they move throughout the building (Figure $6.1 .8-6.1 .9)$.
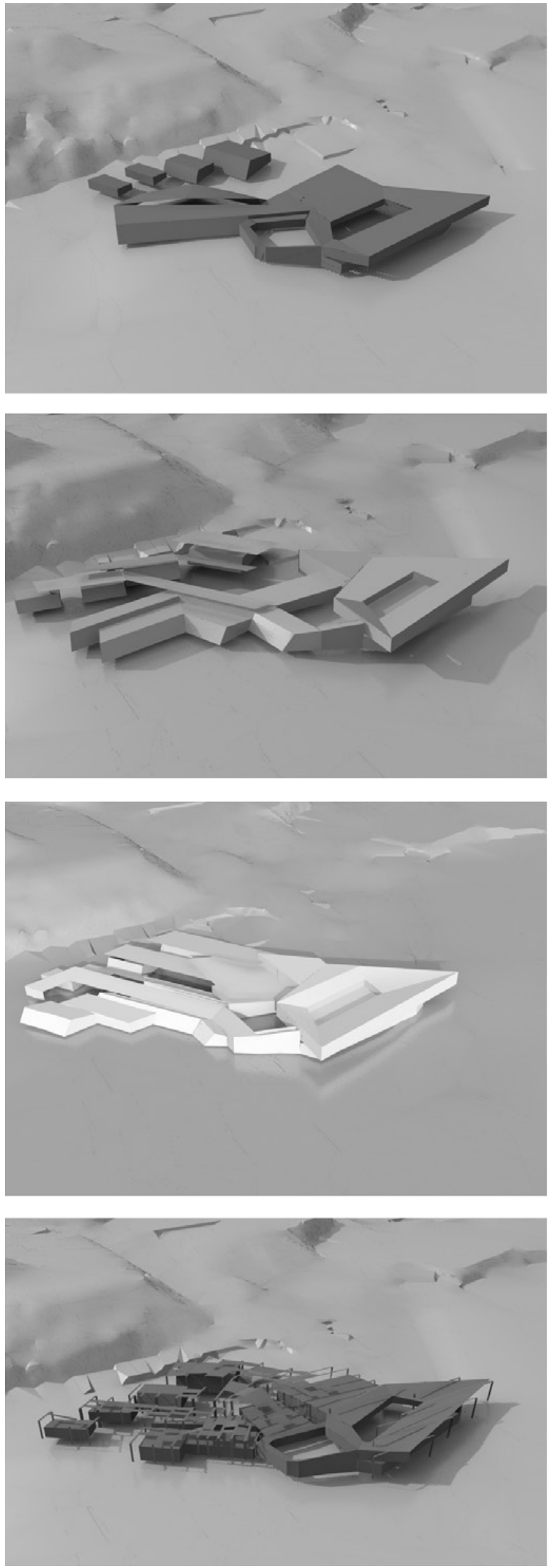

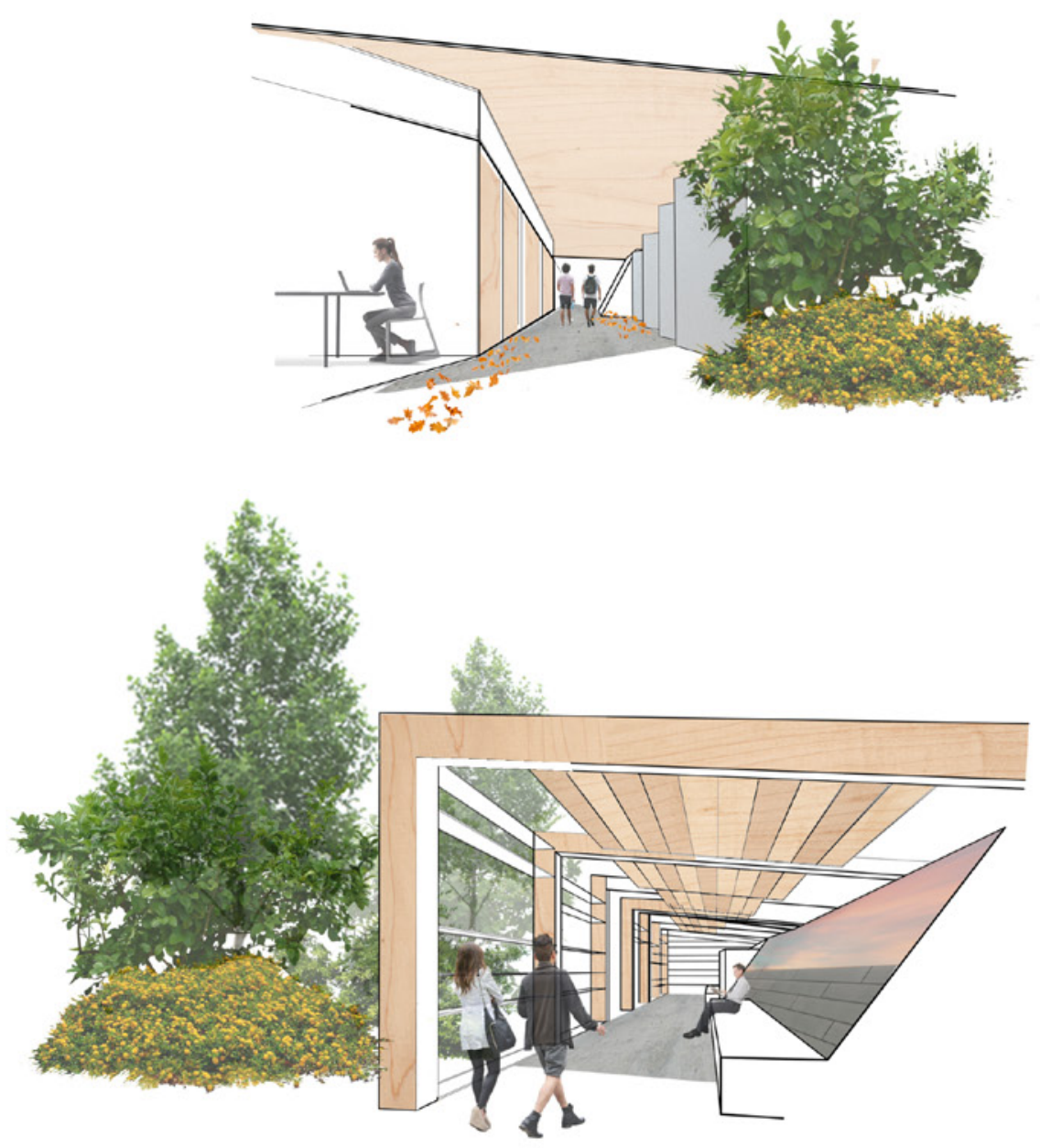

6.1 .8
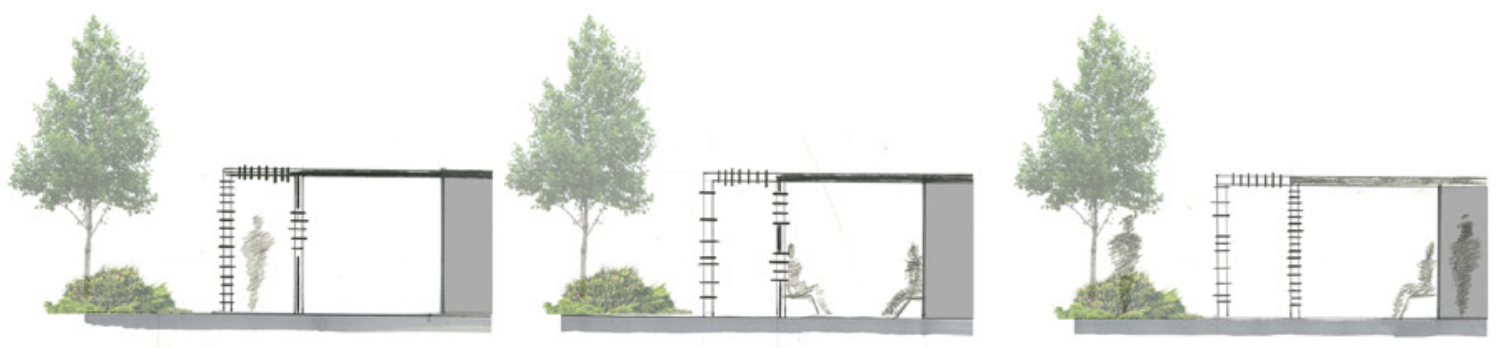

6.1 .9 


\section{Developed Courtyard Model}

\section{Positives}

Promotes an aesthetic of environmental research through the use of daylight technologies

Form allows community to be exposed to outdoor spaces through use of courtyards and louvre canopy, promoting the therapeutic ideals of the environment for visitors to the facility (Figure 6.1.11)

\section{Negatives}

Multiple courtyards limit interaction between residents and community; need to experiment with more communal spaces between both groups

The scale and aesthetic of the louvre canopy would be inappropriate for residents as they arrive to the facility. The exterior form reflecting an institutional rather than domestic quality for residents (Figure 6.1.10)
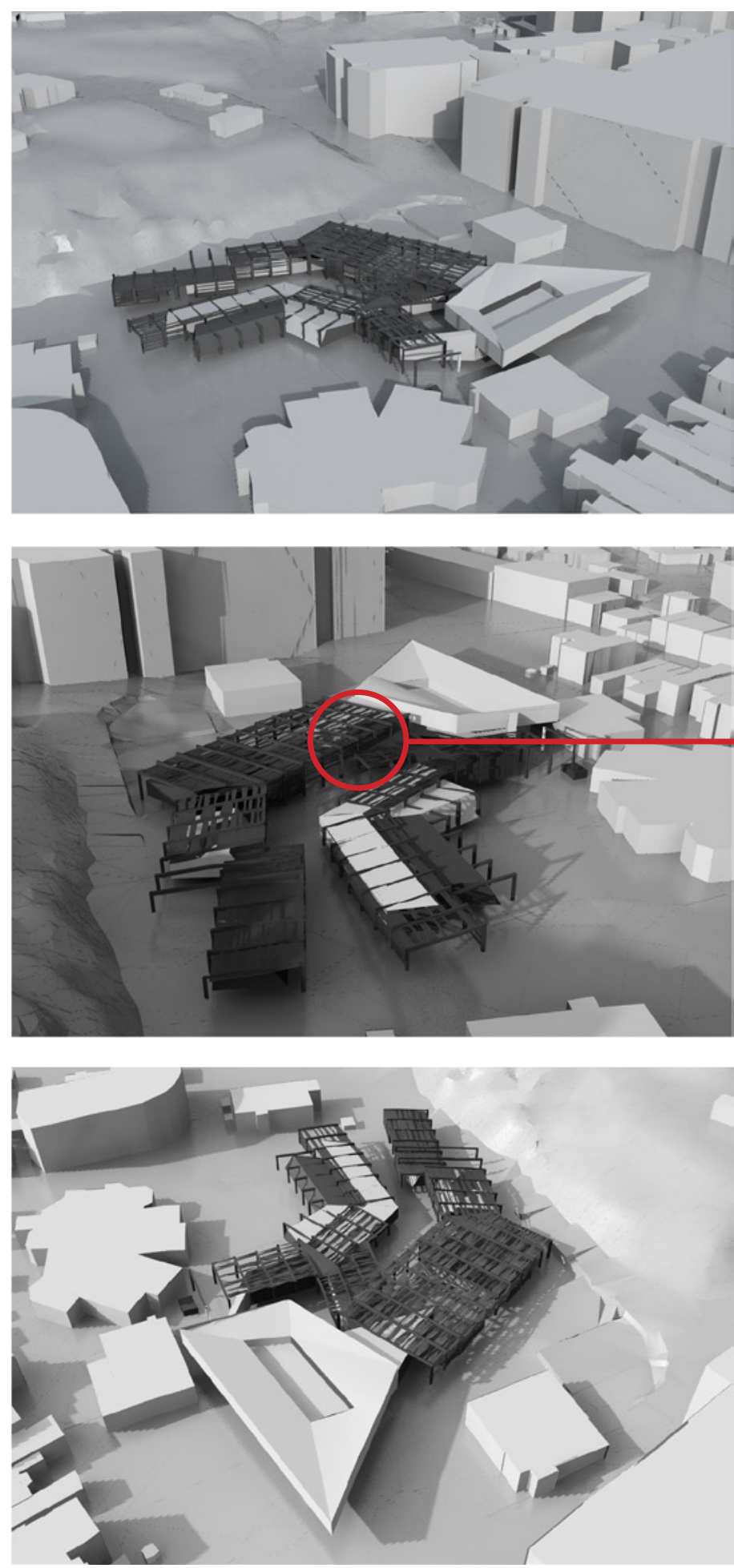

6.1 .10

\subsubsection{Exterior perspectives of courtyard development}

6.1.11 Covered courtyard entrance perspective 


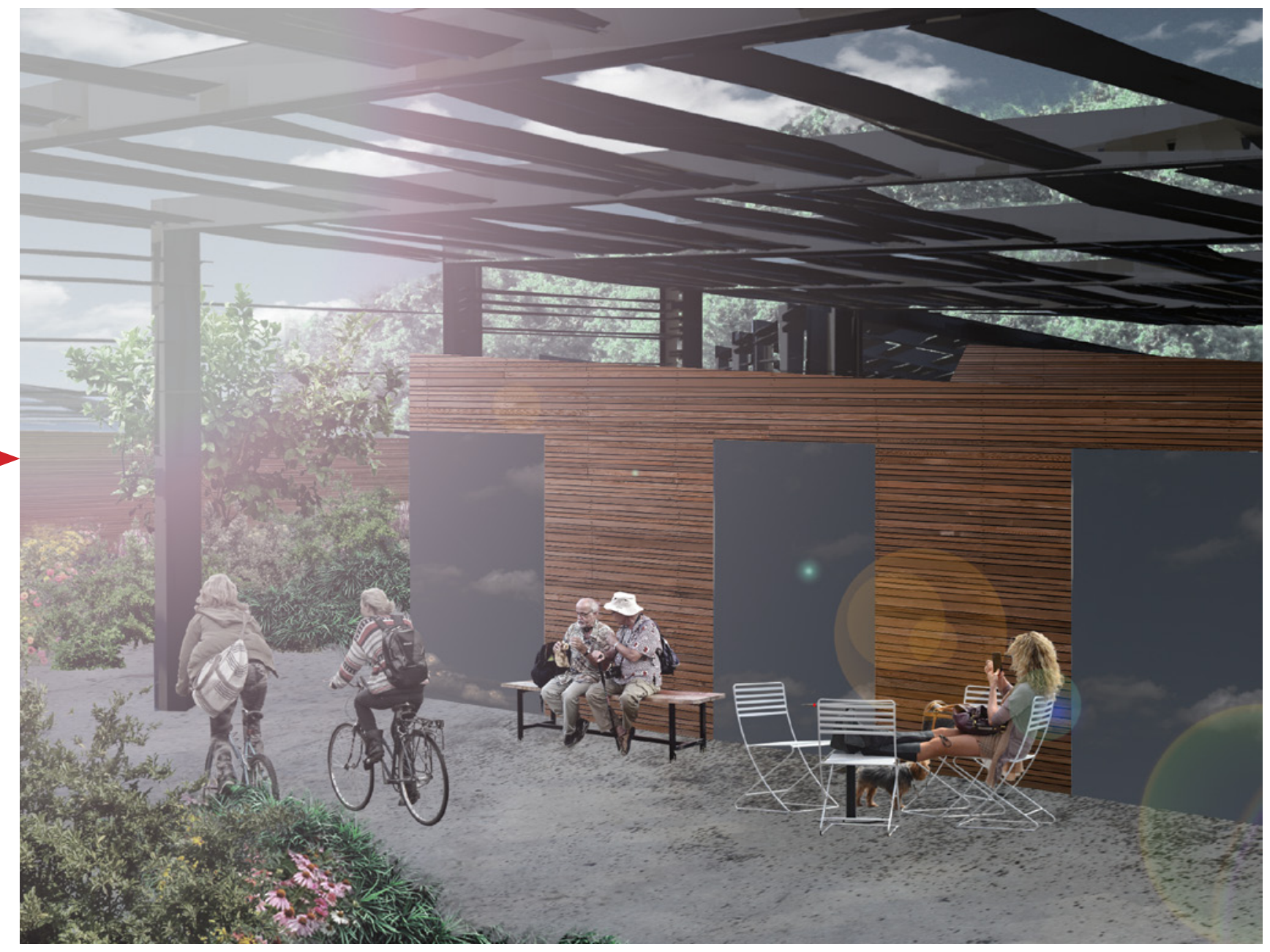

6.1 .11 


\section{Shared Courtyard}

A second series of programmatic arrangements was explored by introducing levels, overlapping programs and elevated walkways to strengthen interactions and social encounters between residents and the medical community (Figure 6.1.12). The notion of a shared courtyard was developed to promote social interaction between the two groups and create a more communal atmosphere (Figure 6.1.13).
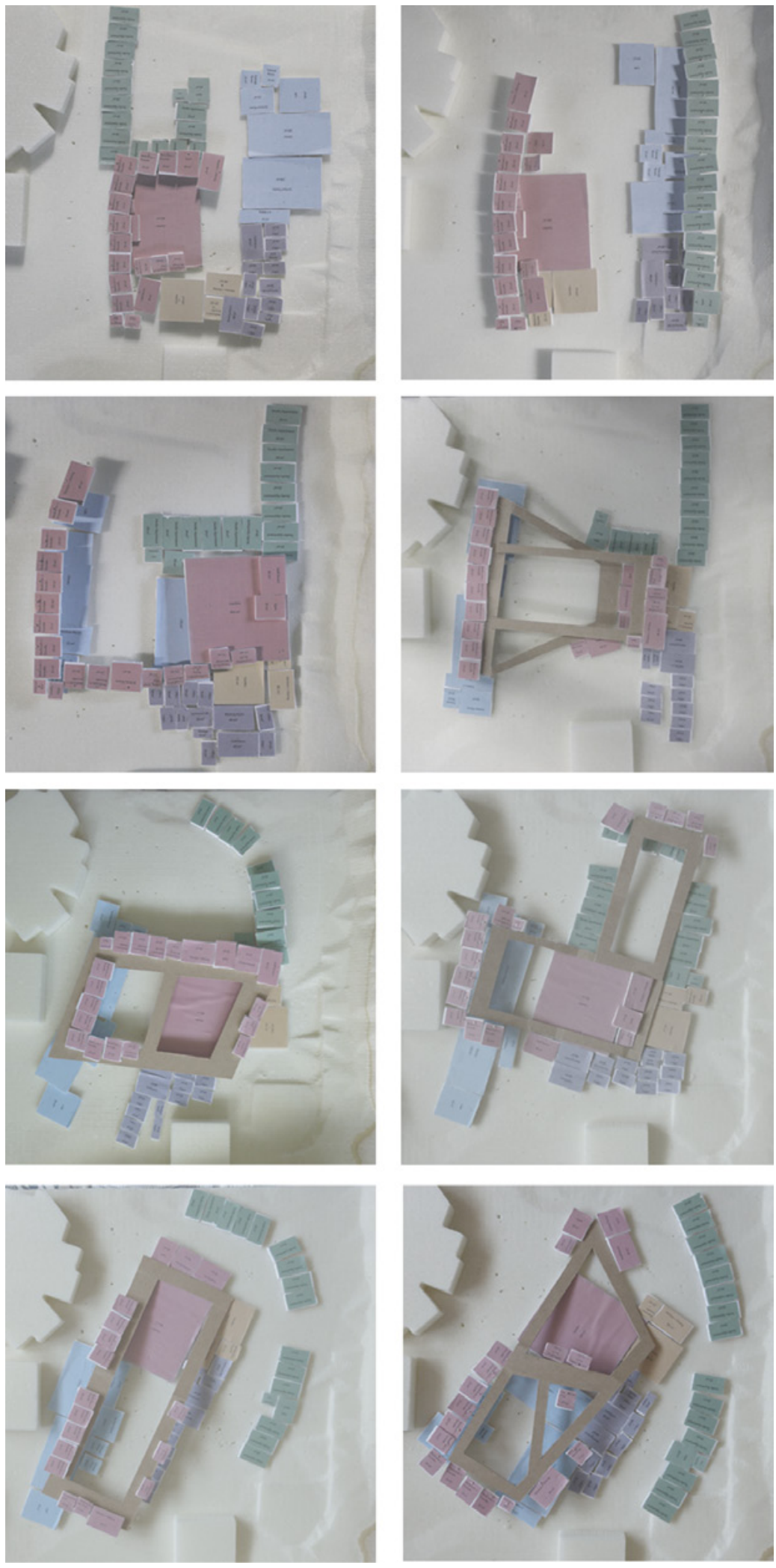

6.1 .12 

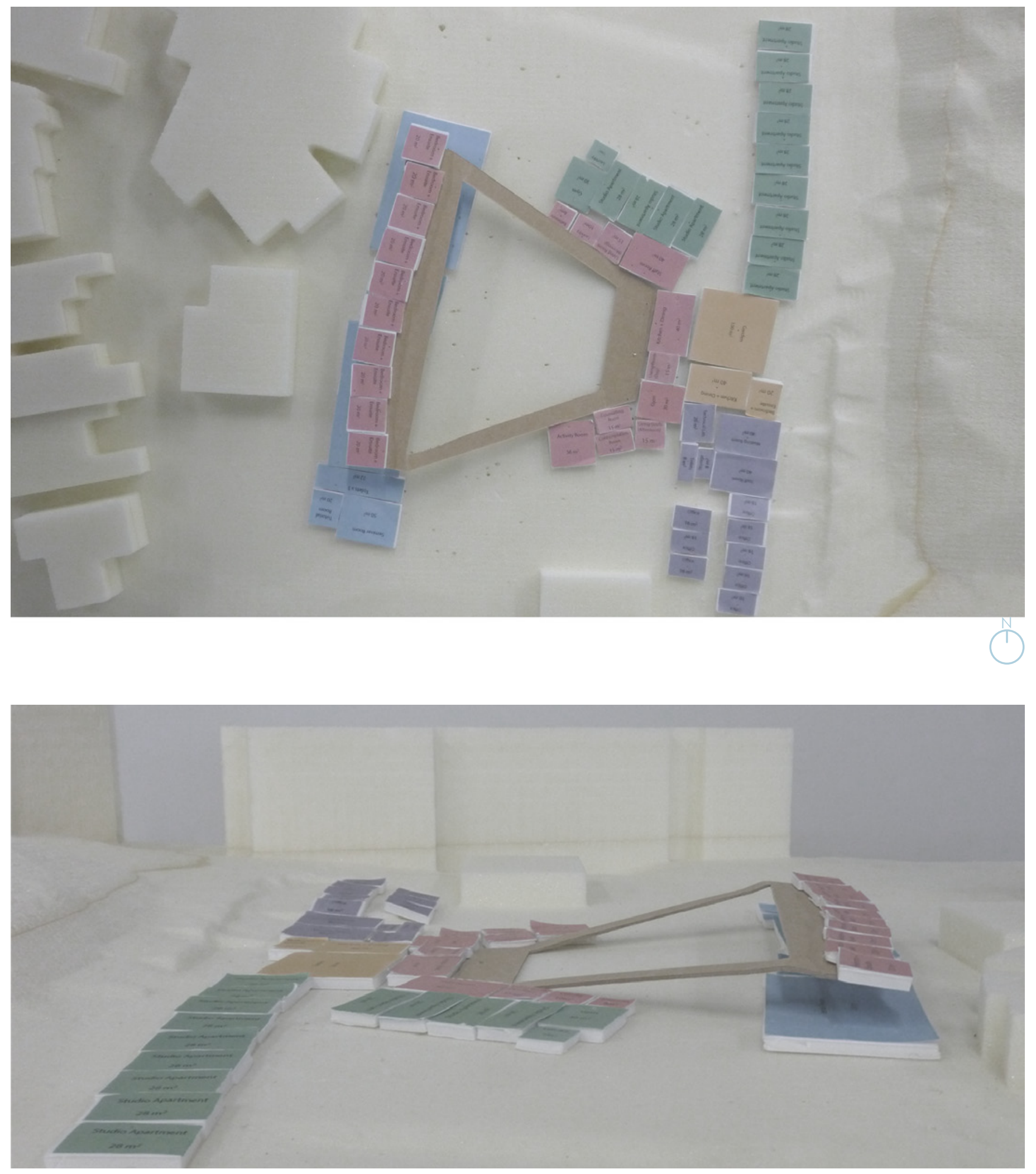

6.1 .13 


\section{Sundial Massing Studies}

Sundial imagery was utilized as a formal driver in these massing studies (Figure 6.1.14) to accentuate movement and circulation amongst residents and the public through a series of open and enclosed courtyard spaces (Figure 6.1.15). Elevated walkways and landscape elements create a secure environment for residents to wander, while also allowing accessibility of the medical community to filter through and occupy the enclosed courtyard space alongside residents (Figure 6.1.16).
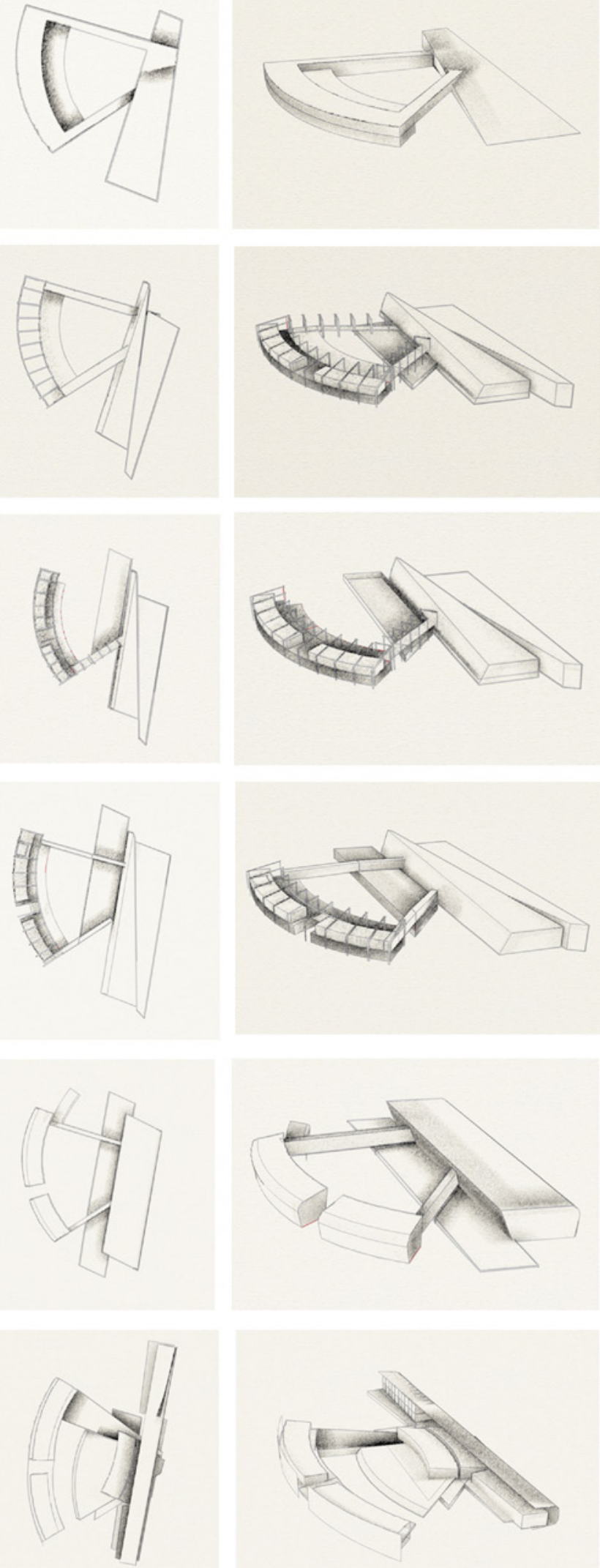

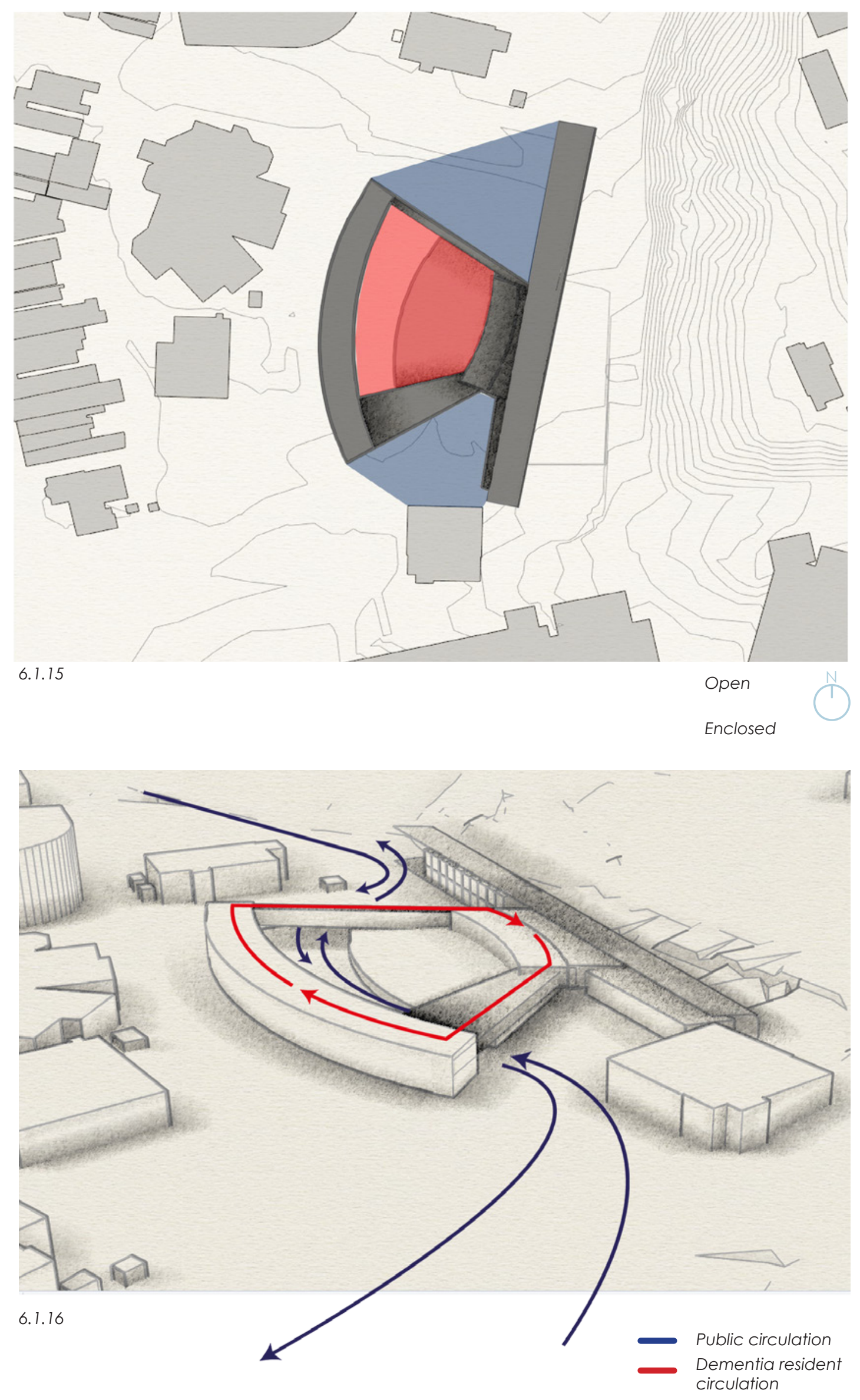


\section{Expressing Light Therapy}

In communicating the purpose of the building towards the medical community, a series of iterative models explored the use of tensile membrane structures to communicate the buildings relationship to natural light (Figure 6.1.17). Utilizing these familiar architectural shading devices helped to communicate this idea and facilitate a public awareness towards the use of light therapy within dementia care environments. The scale and form of these building elements create a stark contrast on site that draws interest within the community.
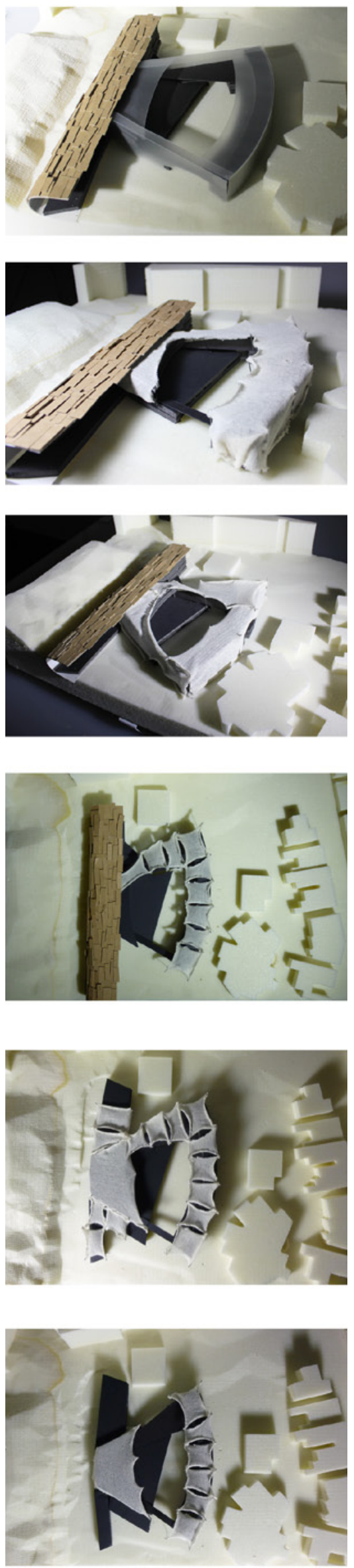

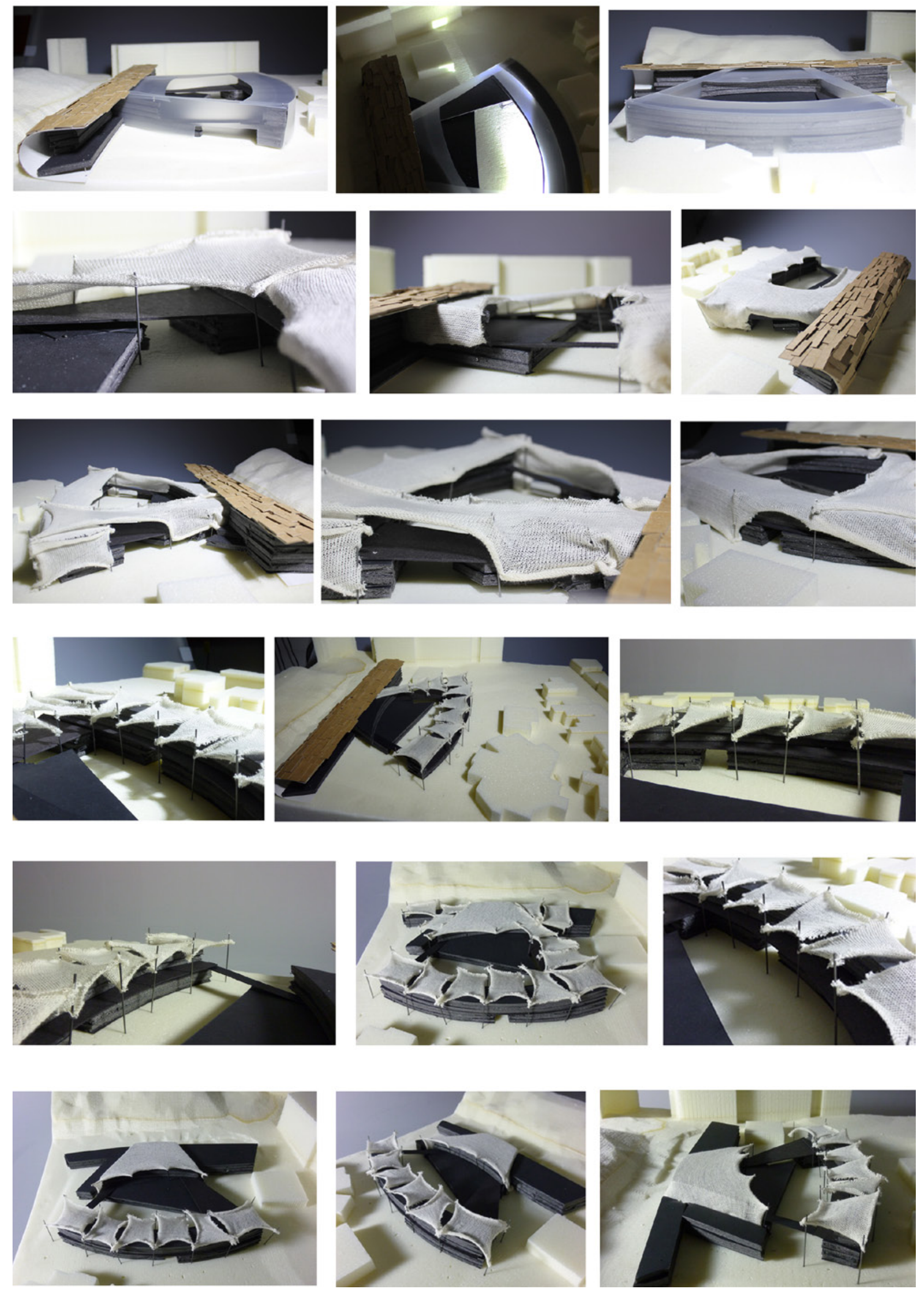


\section{Adjusting for Seasonal Variation}

Knowles theory of the architectural interstitium was explored in the use of the tensile membrane-shading device. Iteration one explored opportunities for the structure to expand and contract to allow for different seasonal envelopes within the courtyard space (Figure 6.1.18). Iteration two utilizes the tensile qualities of the hyperbolic form through orientation and angles to allow for variations in light exposure during different times of the year (Figure 6.1.19), providing a more subtle and appropriate approach to achieving seasonal variation for residents.

Summer$$
\text { ง }
$$

Winter
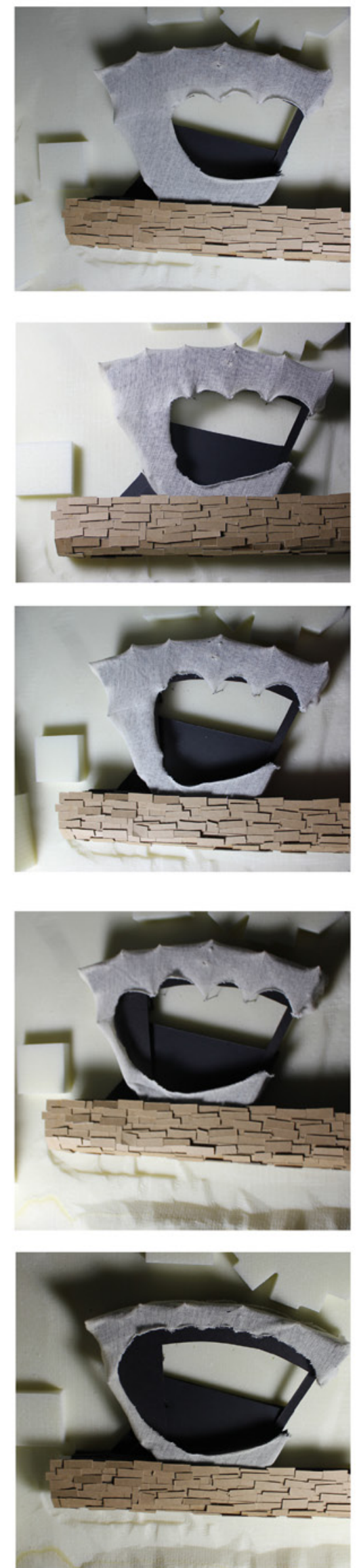

6.1 .18 


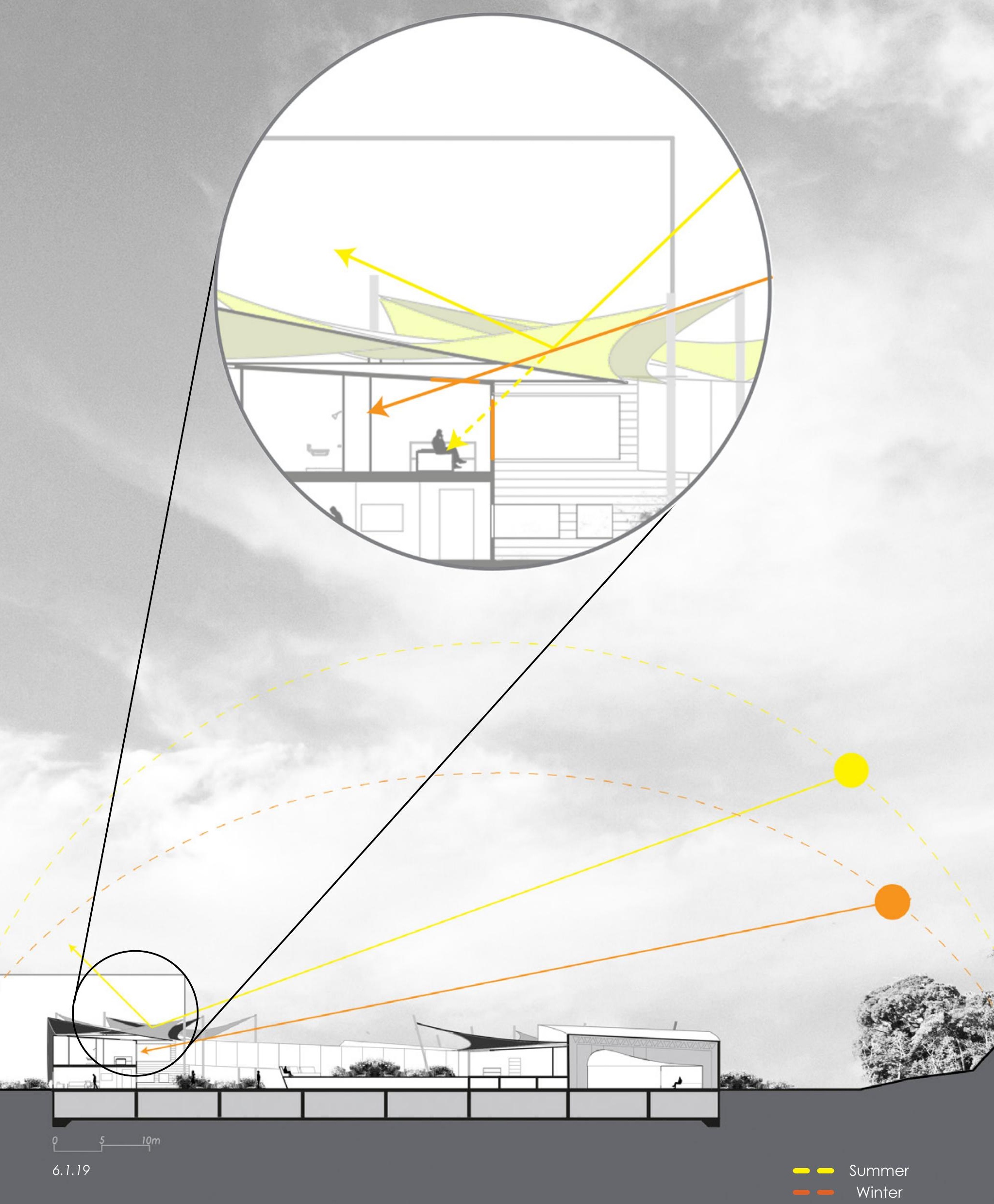




\section{Courtyard Two Development}

\section{Positives}

The form of the design provides a unique contrast to surrounding buildings and promotes a familial aesthetic of light therapy to the surrounding community (Figure 6.1.20 \& 6.1.231

The shared courtyard provides a safe and secure social atmosphere for residents and the medical community

\section{Negatives}

The scale and treatment of the membrane structure deemed to make the building more a spectacle for the public than a tool for therapy (Figure 6.1.21 - 6.1.22)

The building form lacks a legible and familial environment for residents, the need to establish a sense of domesticity for residents is necessary to balance the needs of the two user groups occupying the building

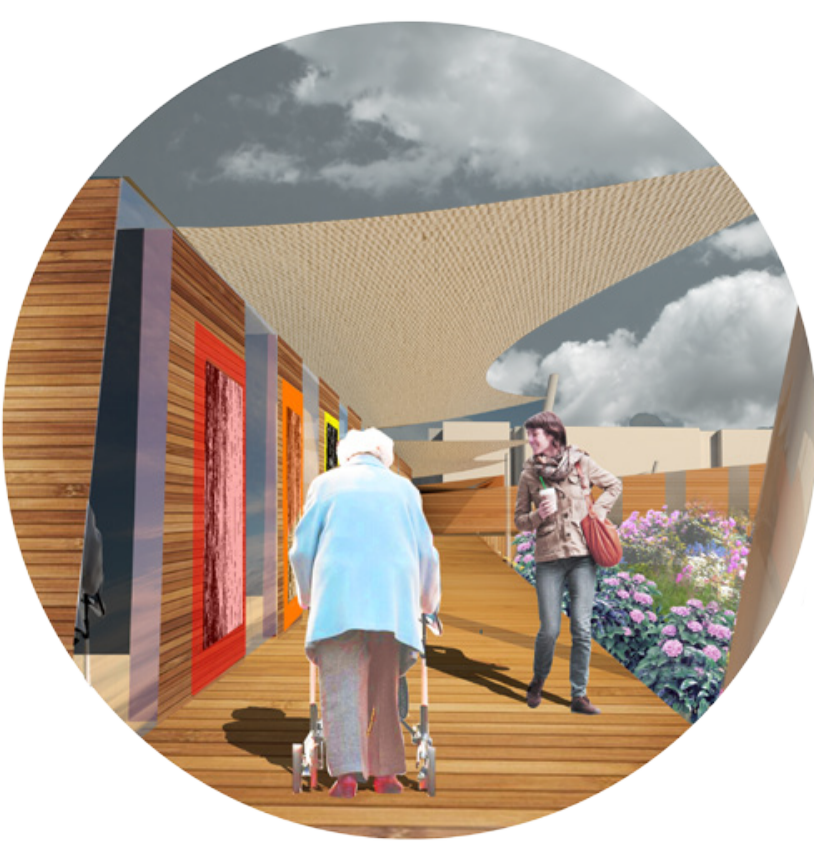

6.1 .20

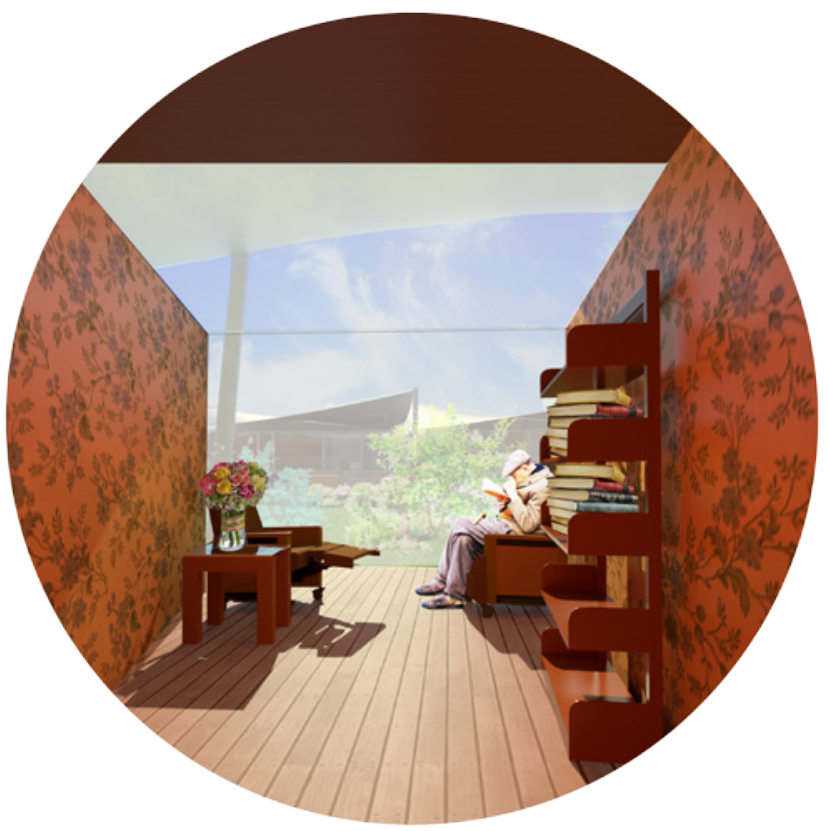

6.1 .21

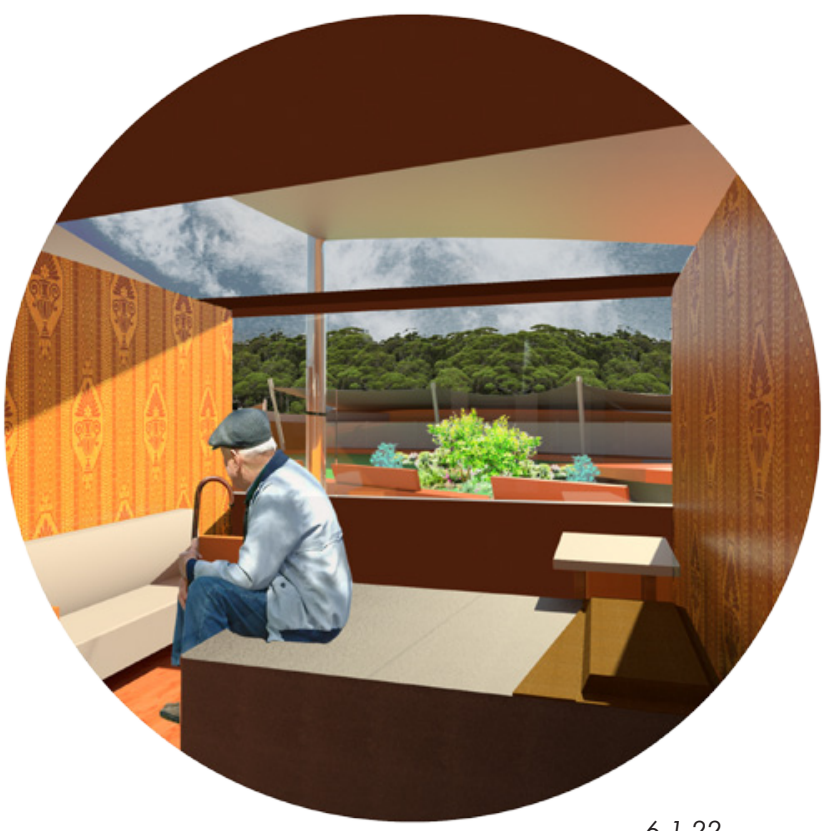

6.1 .22
6.1 .20 Dementia resident entrance
6.1.21 Contemplation room
6.1.22 Bedroom
6.1.23 Exterior perspective 
Courtyard Two Development

(continued)
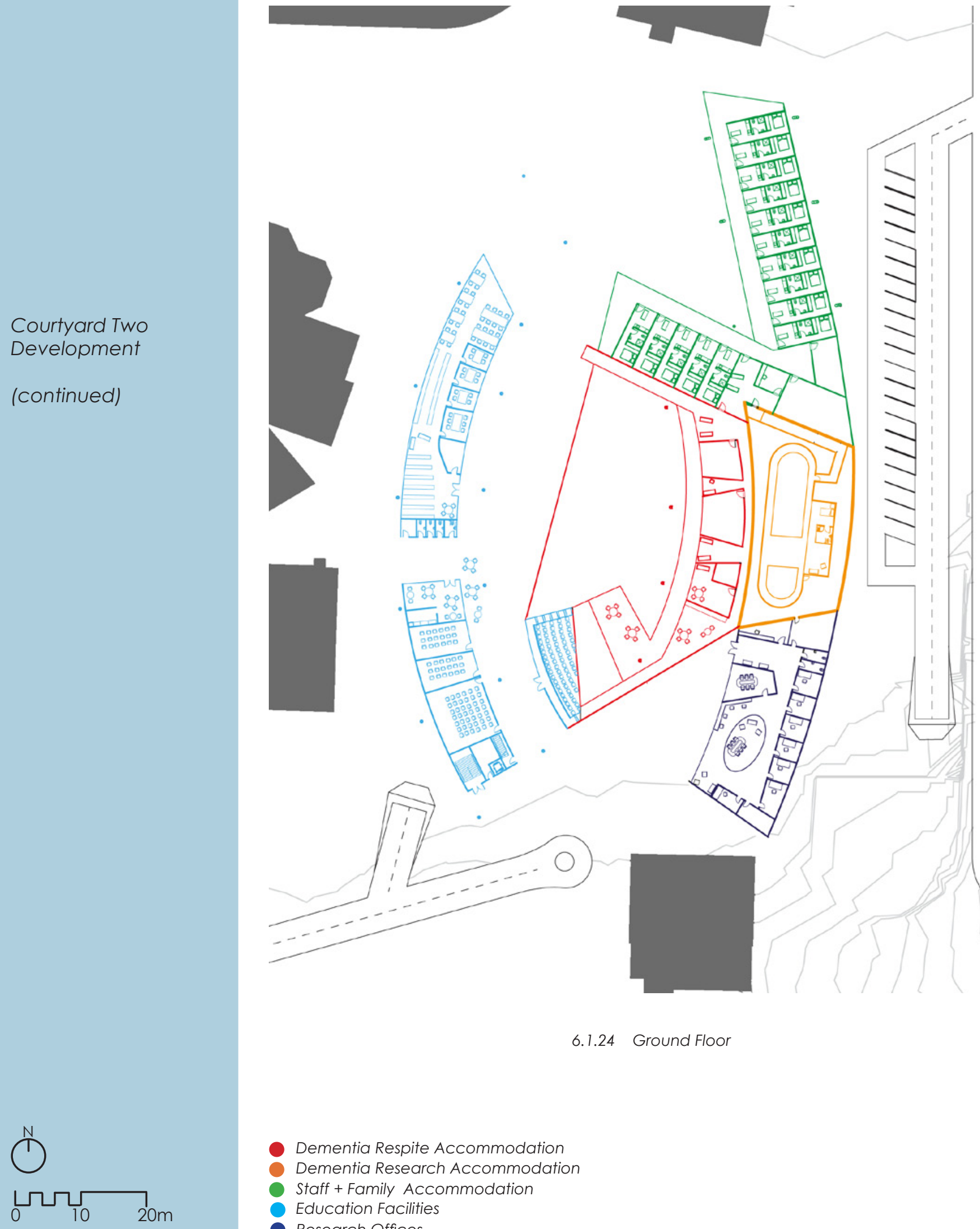

- $10 \mathrm{da}$
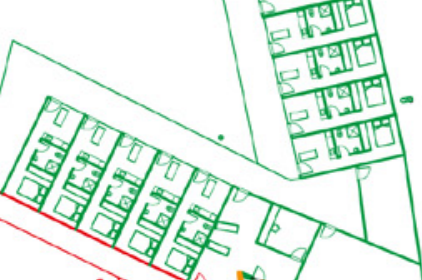

- 12

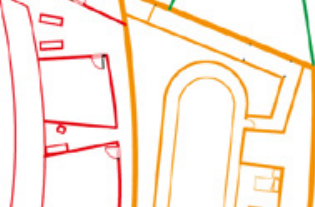

$\cdot$

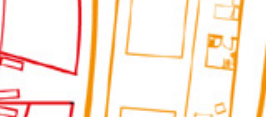

$=$
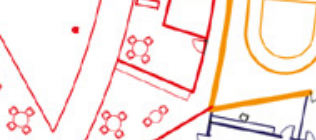

(2)
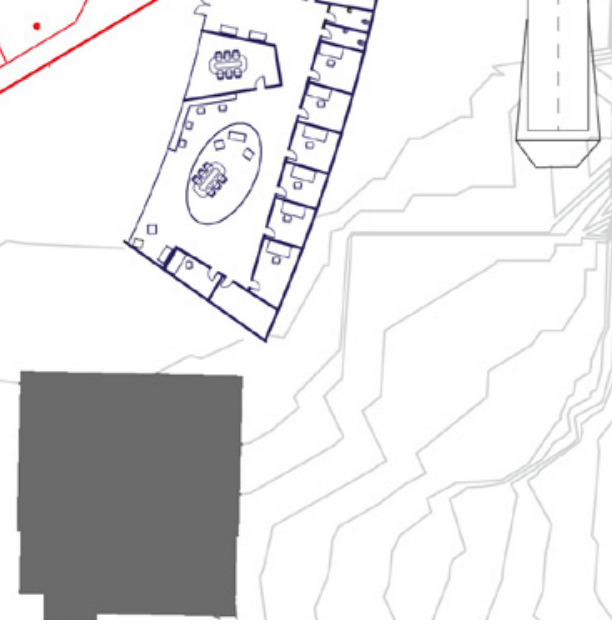

6.1.24 Ground Floor

Dementia Respite Accommodation

Dementia Research Accommodation

Staff + Family Accommodation

Education Facilities

- Research Offices 


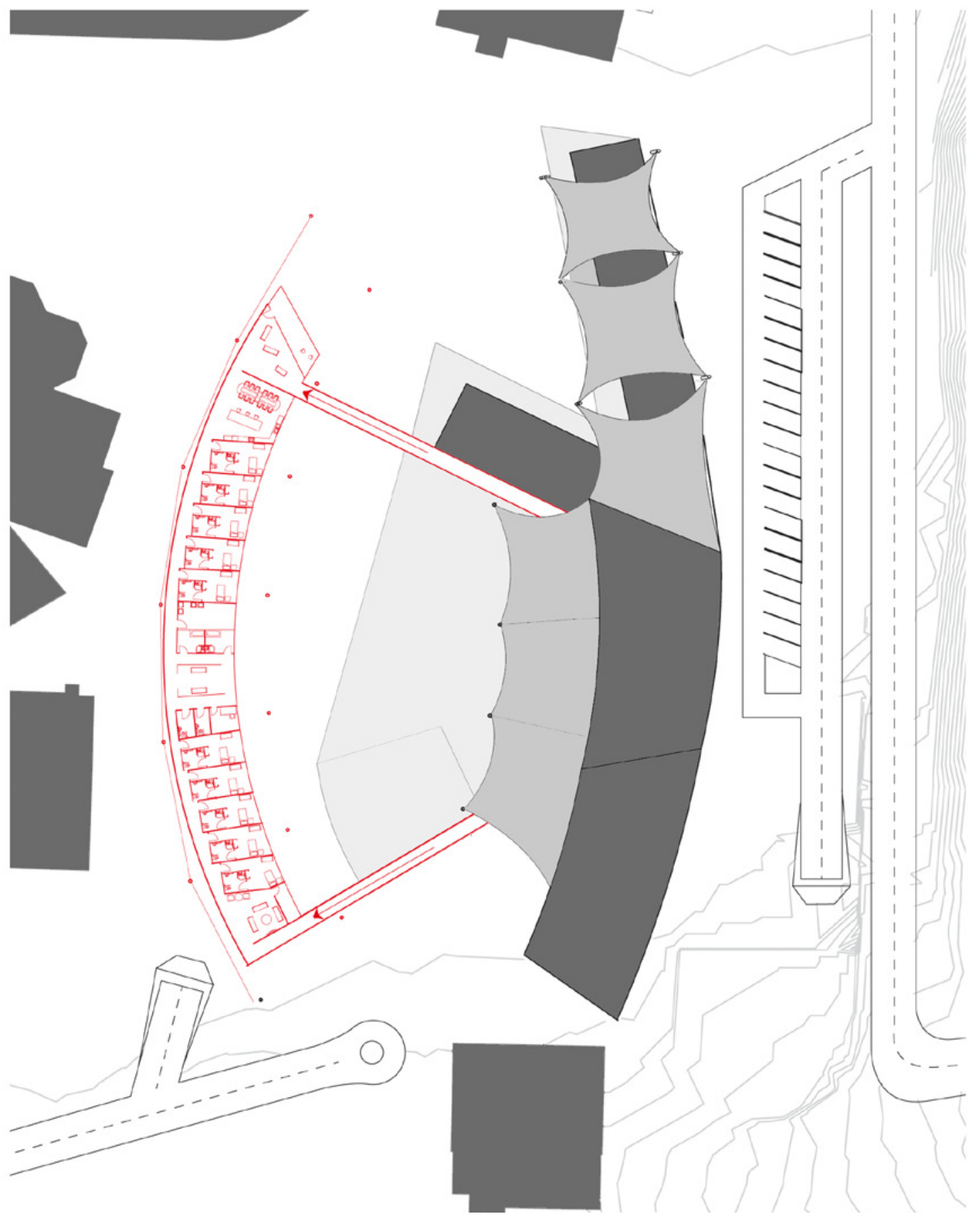

6.1.25 First Floor 


\section{The Edge}

The security benefits of landscape elements such as a ha-ha wall were adopted to maintain a secure boundary between residents and the public within this shared outdoor space (Figure 6.1.27-6.1.28) . Smallscale iterative models explored opportunities to promote social interaction along the boundary and reduce the feeling of confinement for residents within the shared courtyard (Figure 6.1.26)
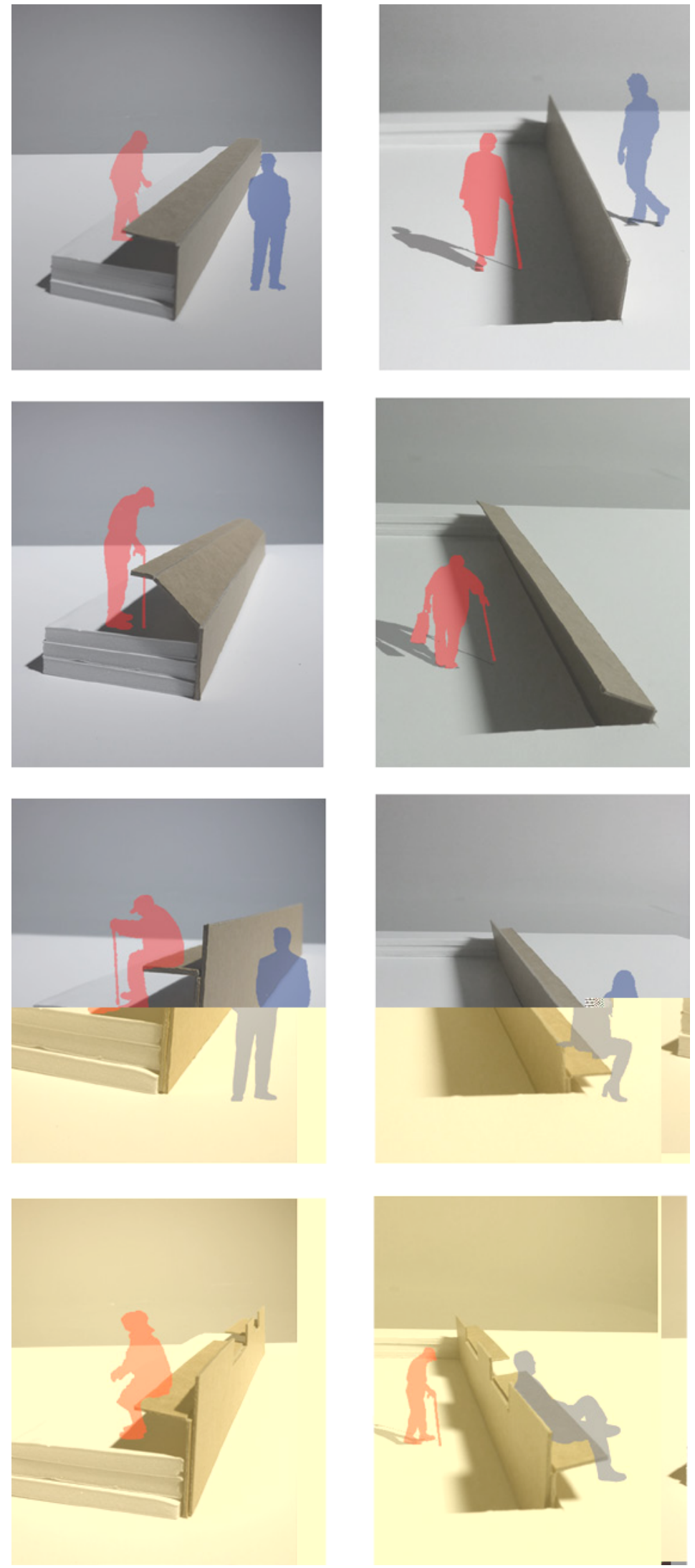

6.1 .26
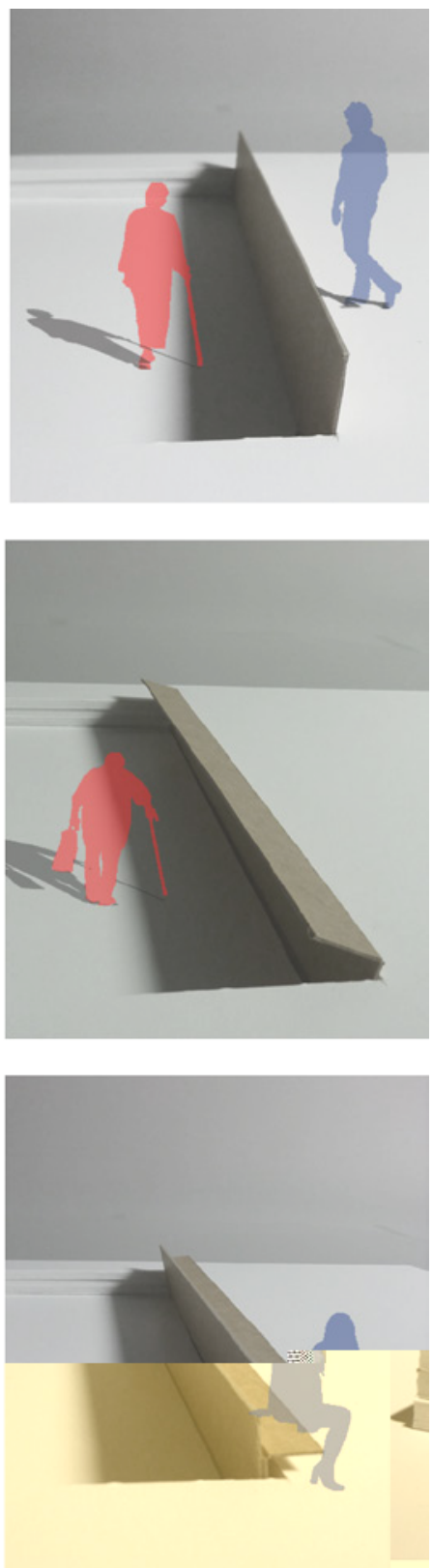

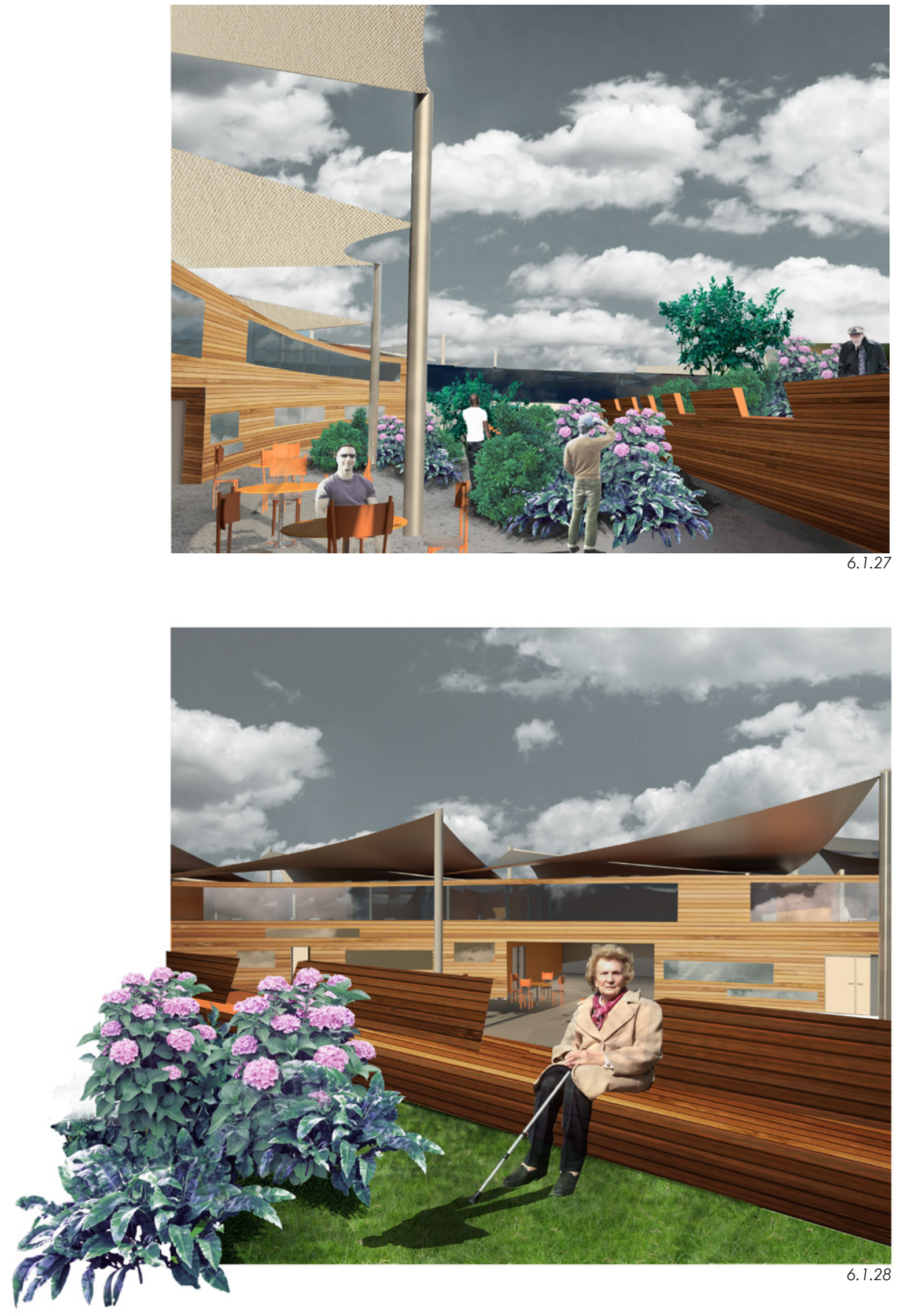


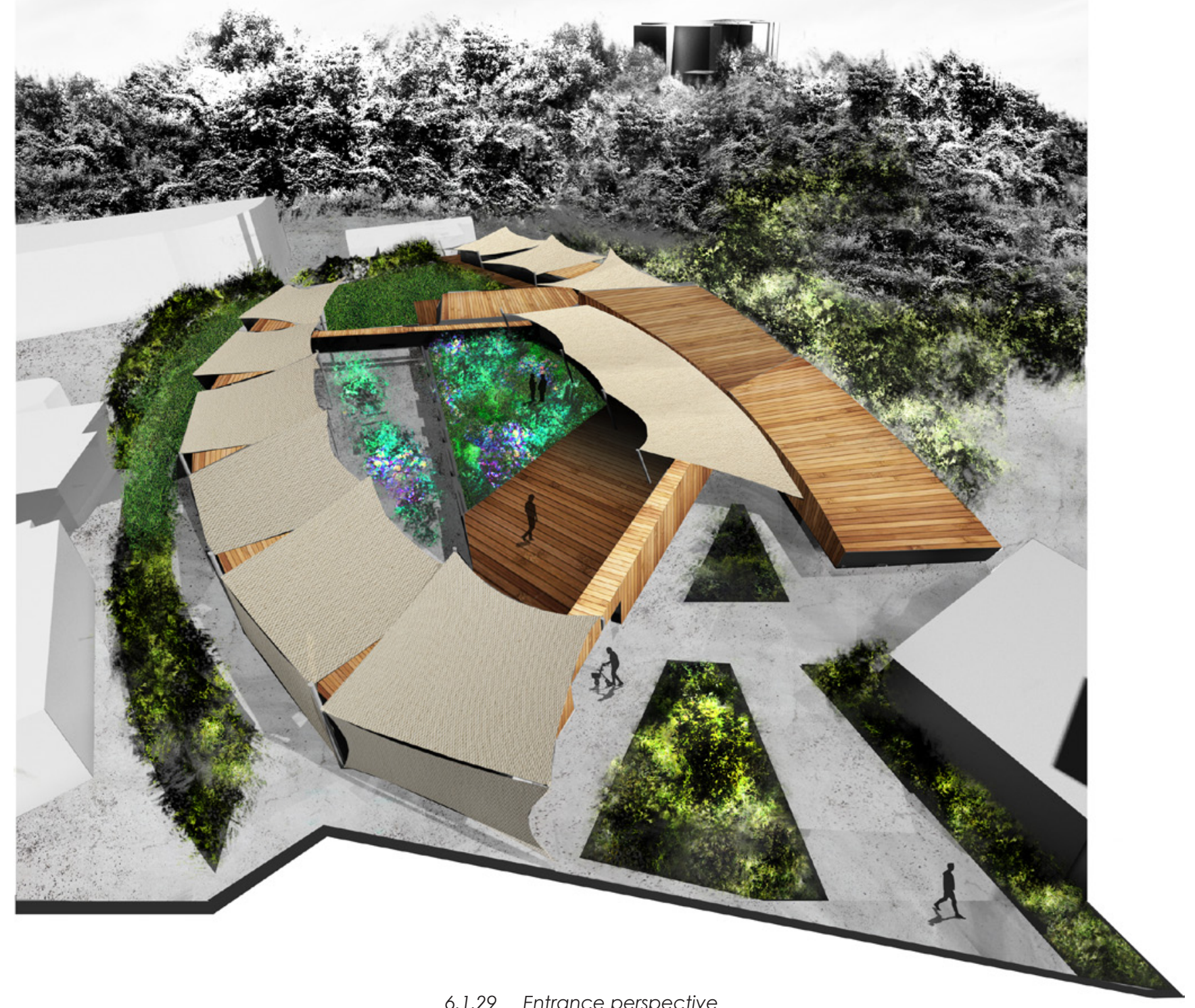




\section{Reflection + Summary}

Design phase one tested an appropriate response towards establishing design principles to help integrate the medical community and dementia residents within a single building. The development of a shared courtyard to promote a social space for residents and the medical community was considered successful. Similarly, the contrast in form to surrounding buildings helped to establish the facility as point of interest on site, the exterior aesthetic promoting the notion of environmental therapy for dementia to the public. While architectural elements such as louvres and tensile member structures promote an aesthetic of light therapy for dementia, the subsequent scale and form of these architectural elements created an institutional aesthetic that reduced their effectiveness for dementia residents. The design phase highlighted the apparent conflict in balancing the needs of both groups within a single facility, with the design favouring the interest of the medical community. The need to explore the design objectives through the perspective of a dementia resident is necessary if the design is to improve environmental therapy for their benefits.

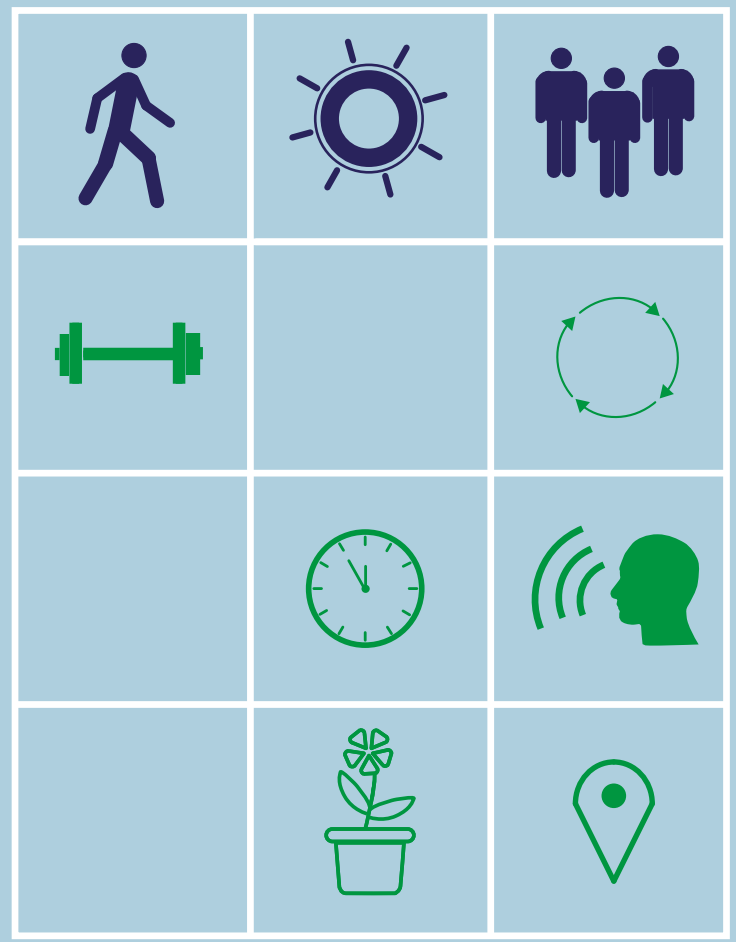




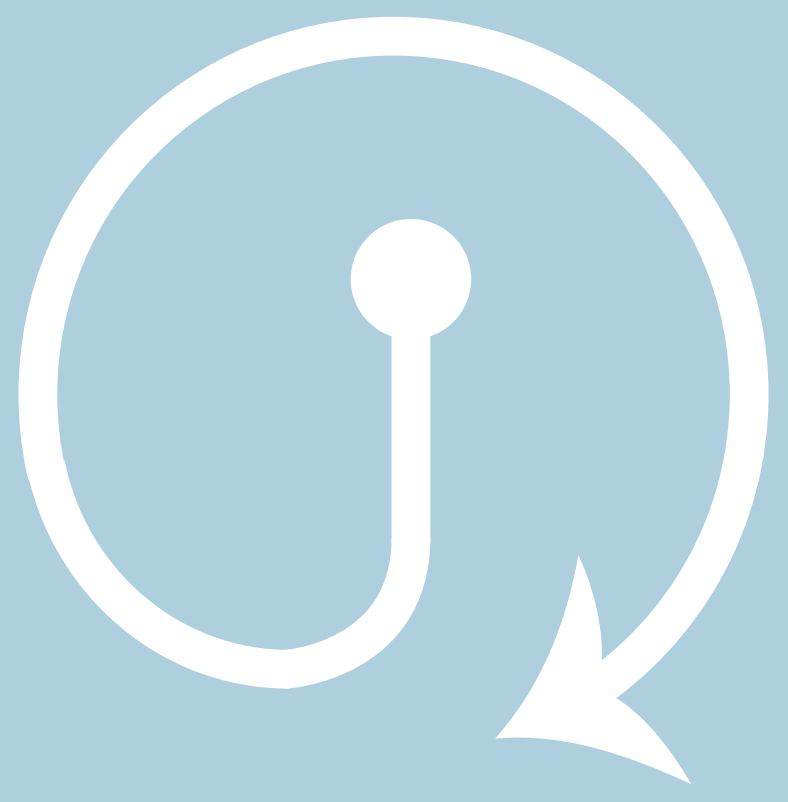




\section{2}

\section{Design Phase 2 \\ "Inside-Out"}

The following design phase tests design methods from the perspective of a dementia resident to help facilitate a design that caters to their desired sensations and experiences to improve their wellbeing during respite. 


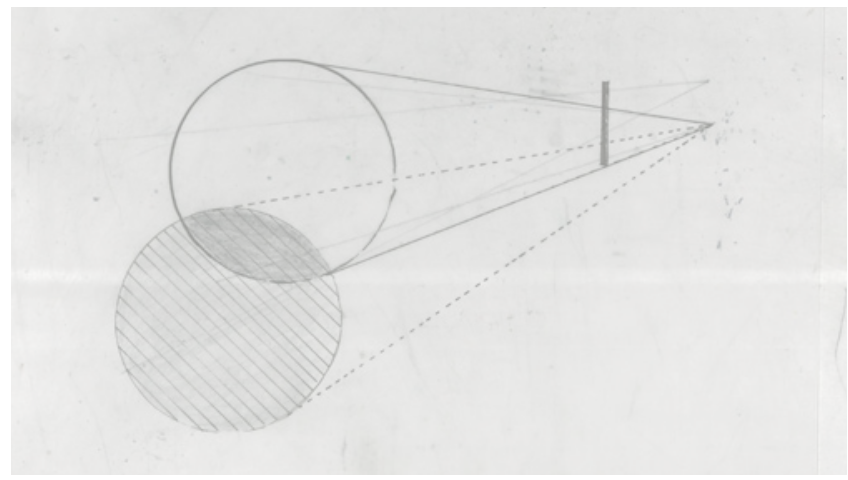

Key views

\section{The Bedroom}

The significance of the bedroom for morning light therapy was identified within the literature review and the starting point for the design process. The proposed bedroom layout tested orientation strategies for the resident lying in bed, identifying visual access to the external environment and the toilet as key views for residents while resting (Figure 6.2.1).

To improve natural light exposure within the bedroom, a series of models tested the use of skylights that would account for seasonal variability. The scale, form and orientation of skylights tested the ability to control natural light exposure for morning stimulation (Figure 6.2.2). The use of mechanical louvres explored a controlled response to seasonal variation, as well as initiating movement by reducing light within the space (Figure 6.2.3). However a key limitation to using louvres involved the casting of shadows, which has implications towards a dementia resident's perspective of space and void.

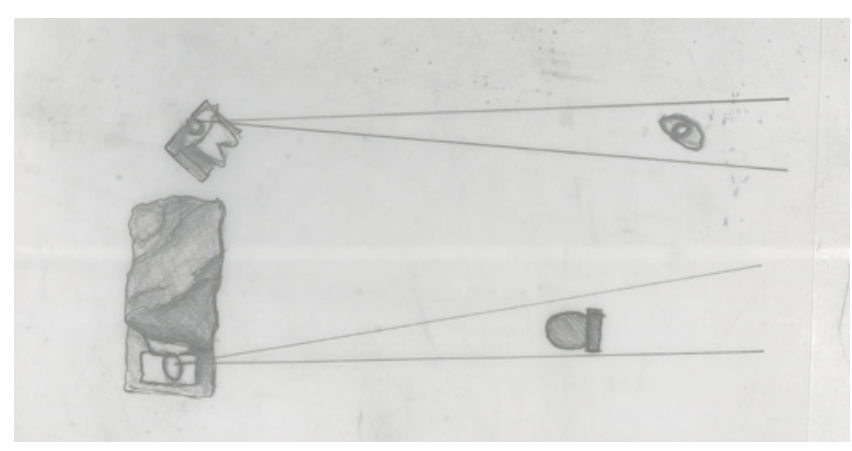

Gurney accessibility

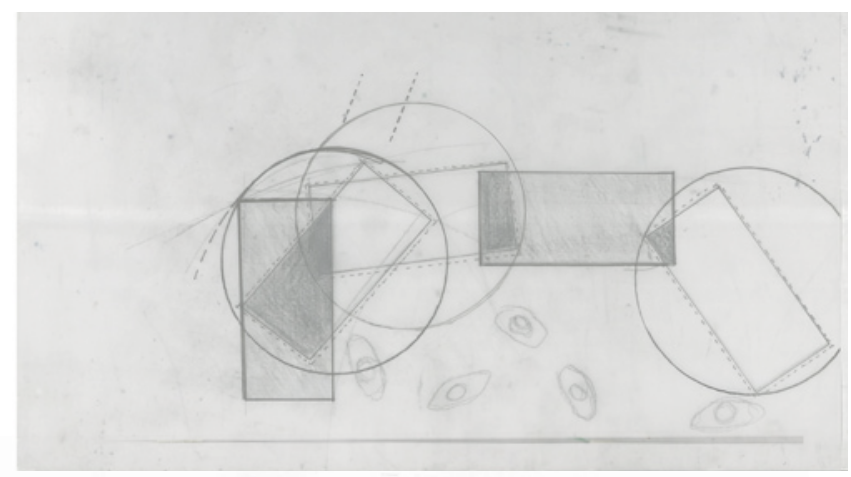

Bedroom Layout

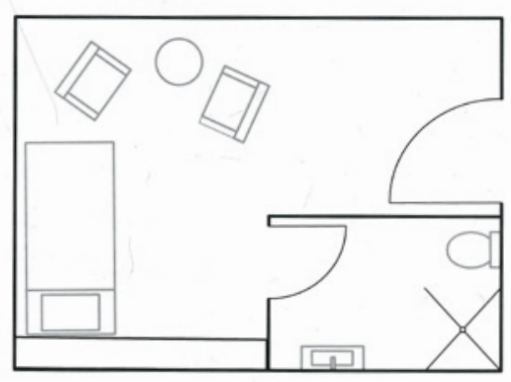

6.2 .1 

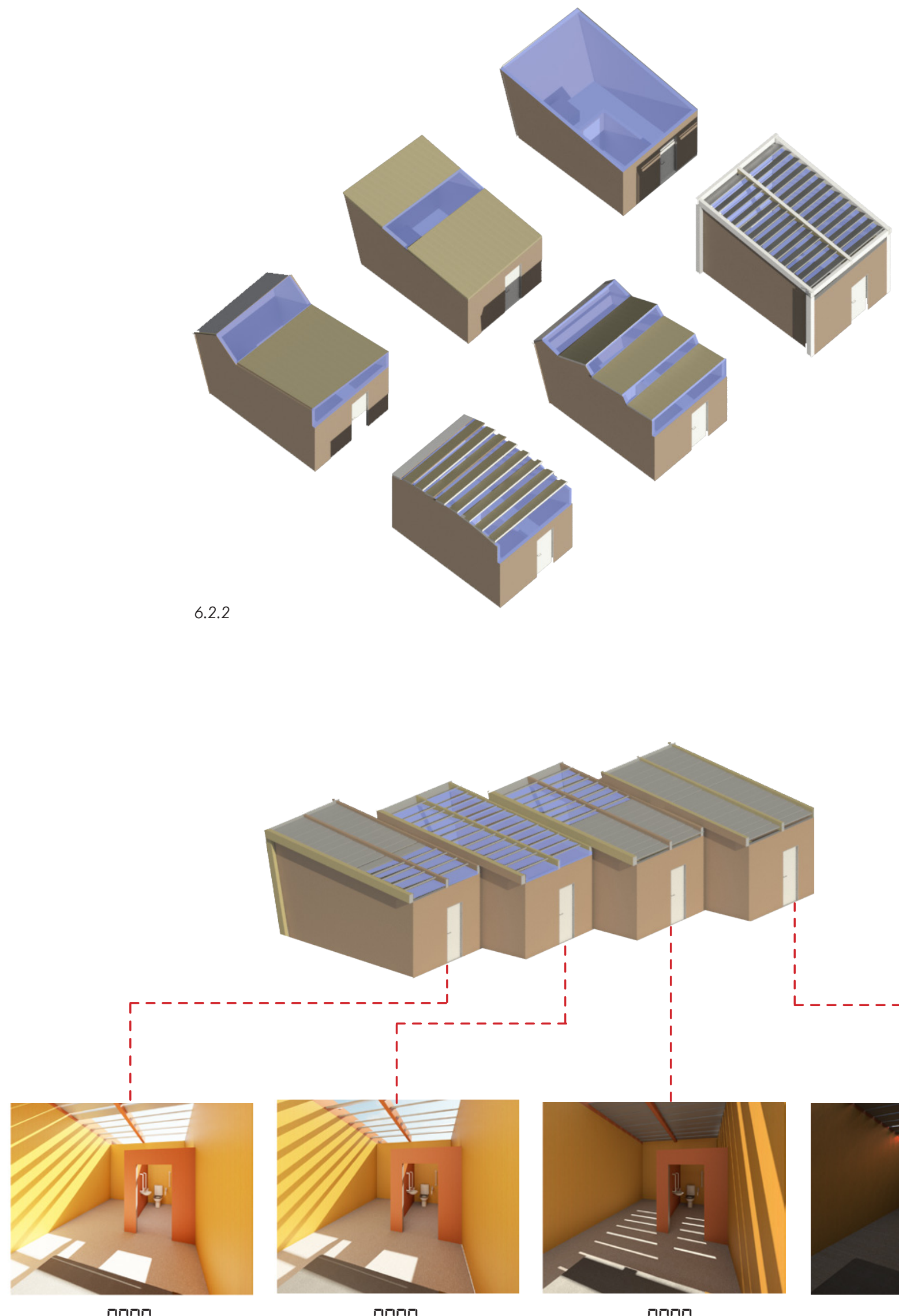

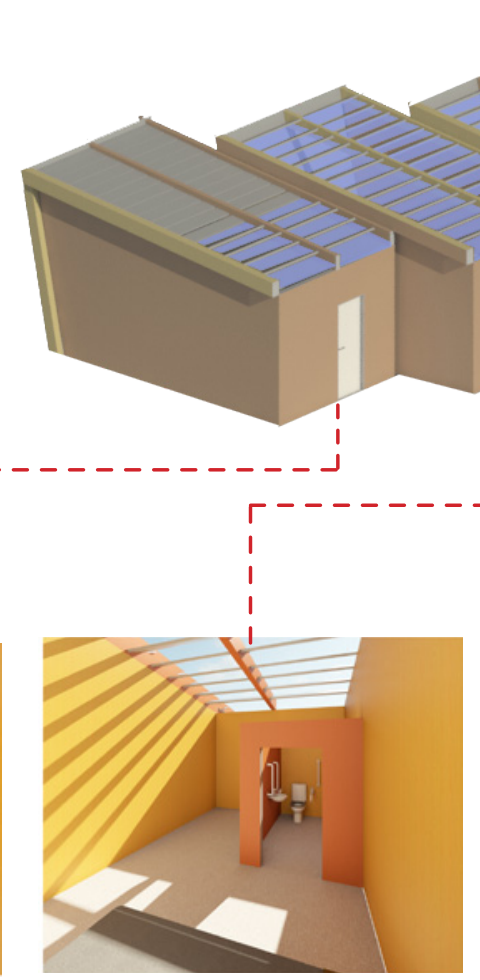

매든

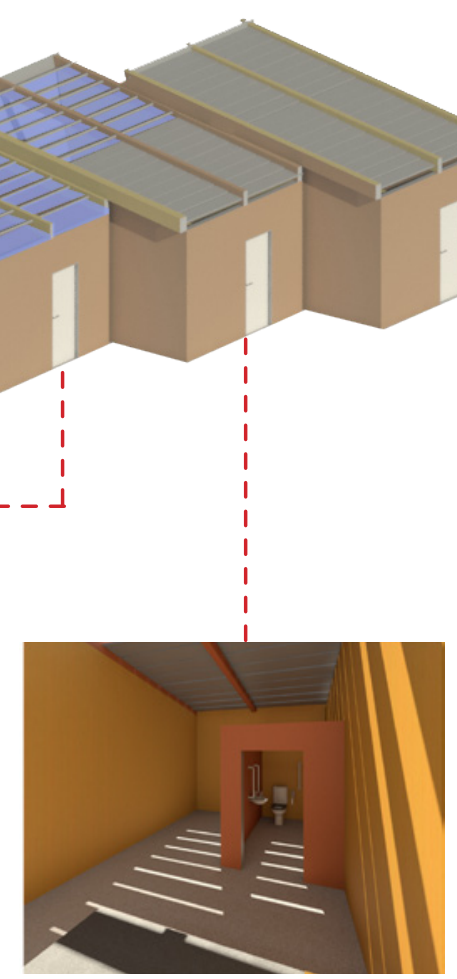

090

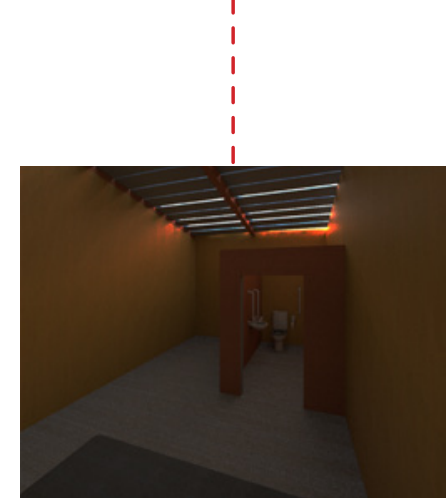

갬ㄷㅁ

6.2 .3 
Down light

Down light 2

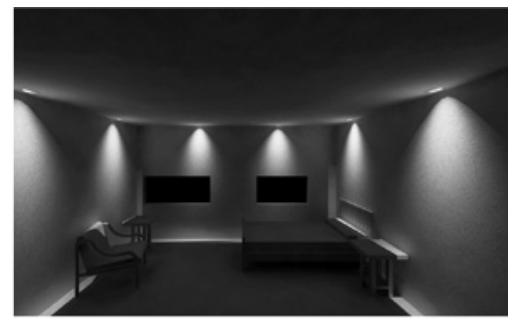

\section{Artificial vs Natural Stimulation}

Sconce light

Windows orientated east for morning light were tested according to scale and placement on the wall to increase the efficiency of natural light exposure for residents in bed (Figure 6.2.5 - 6.2.6). Artificial lights were tested to account for seasonal variation, balancing the need for light stimulation as well as a resident's reduced visual acuteness, with a combination of down lights and spot lights providing the most appropriate response towards a residents primary orientation (Figure 6.2.4).

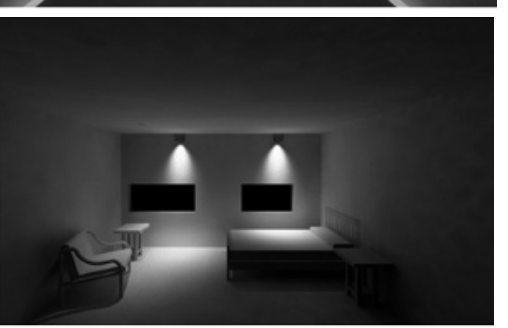

Lamp fixtures

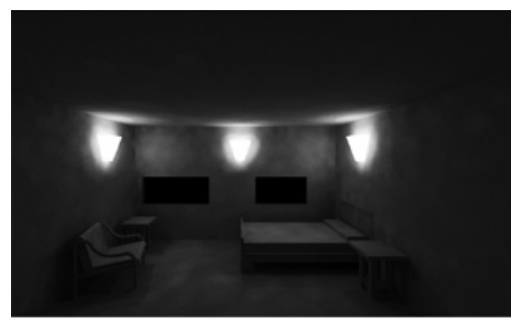

Spot light

Ceiling light
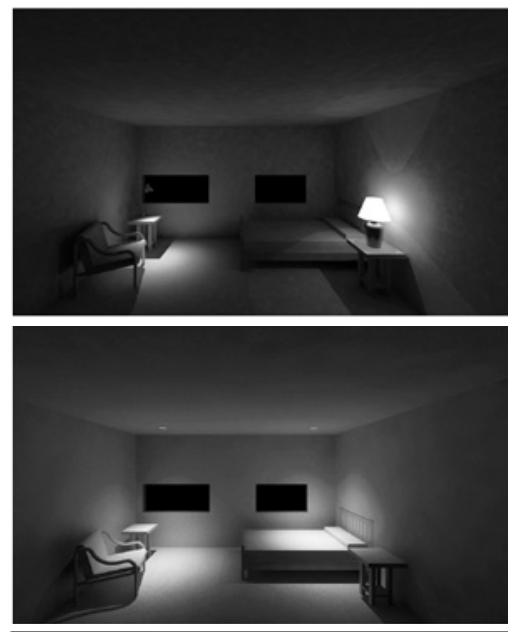

Lamp fixture + Ceiling light

Down light + spot light
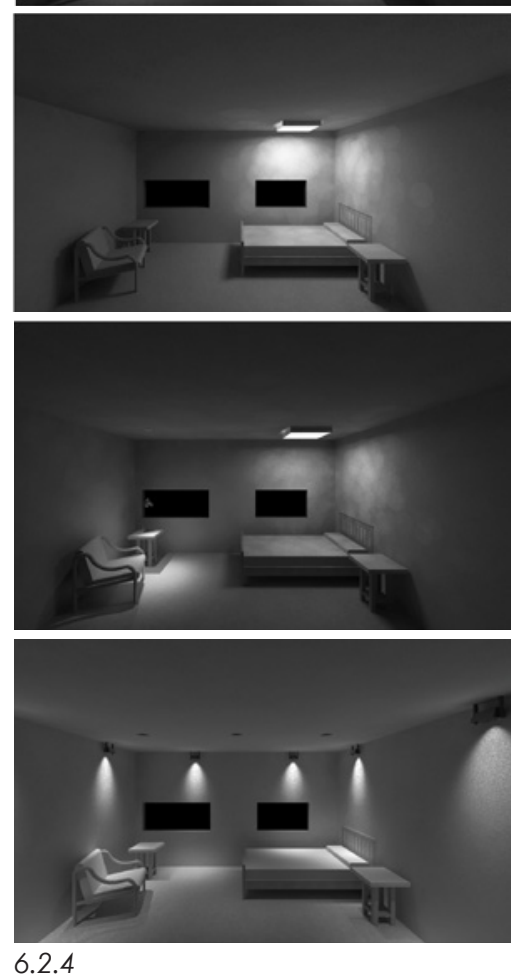


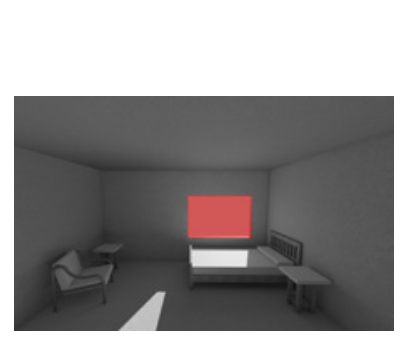

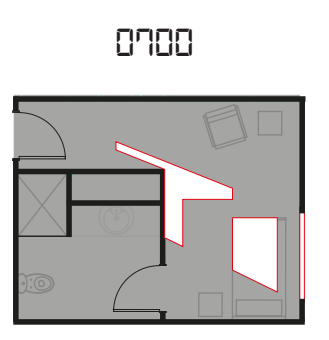
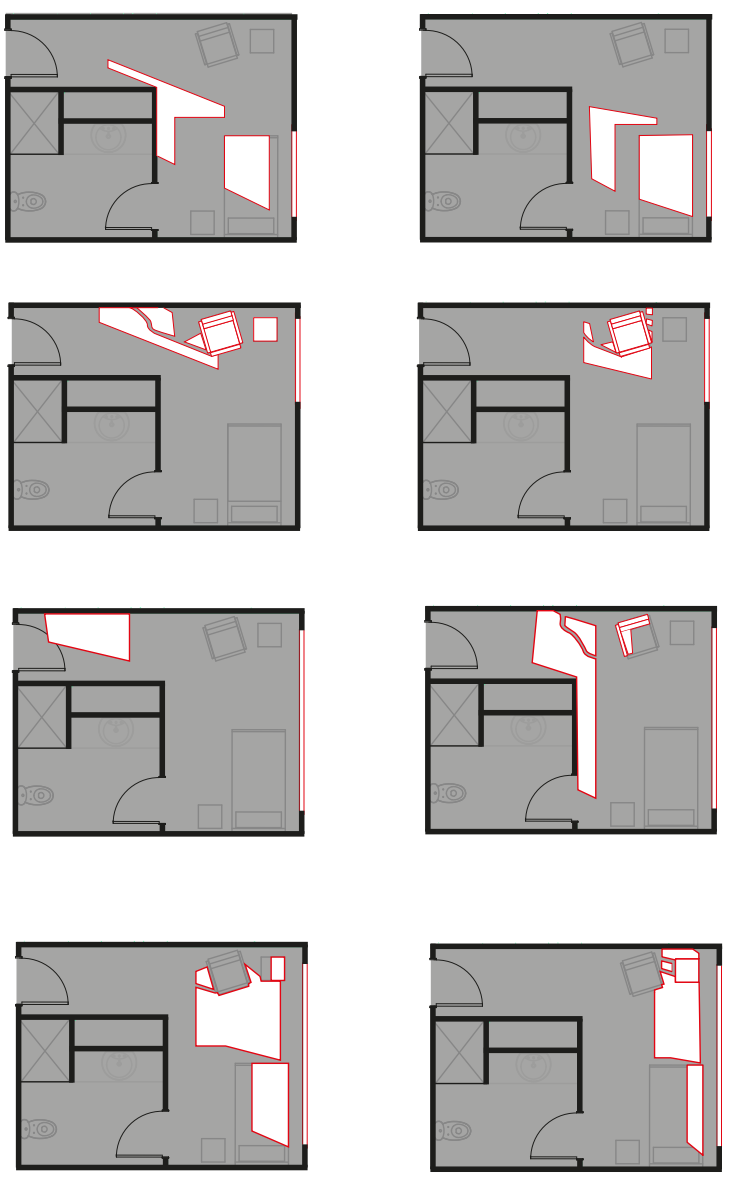

590

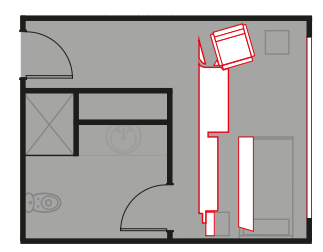

890
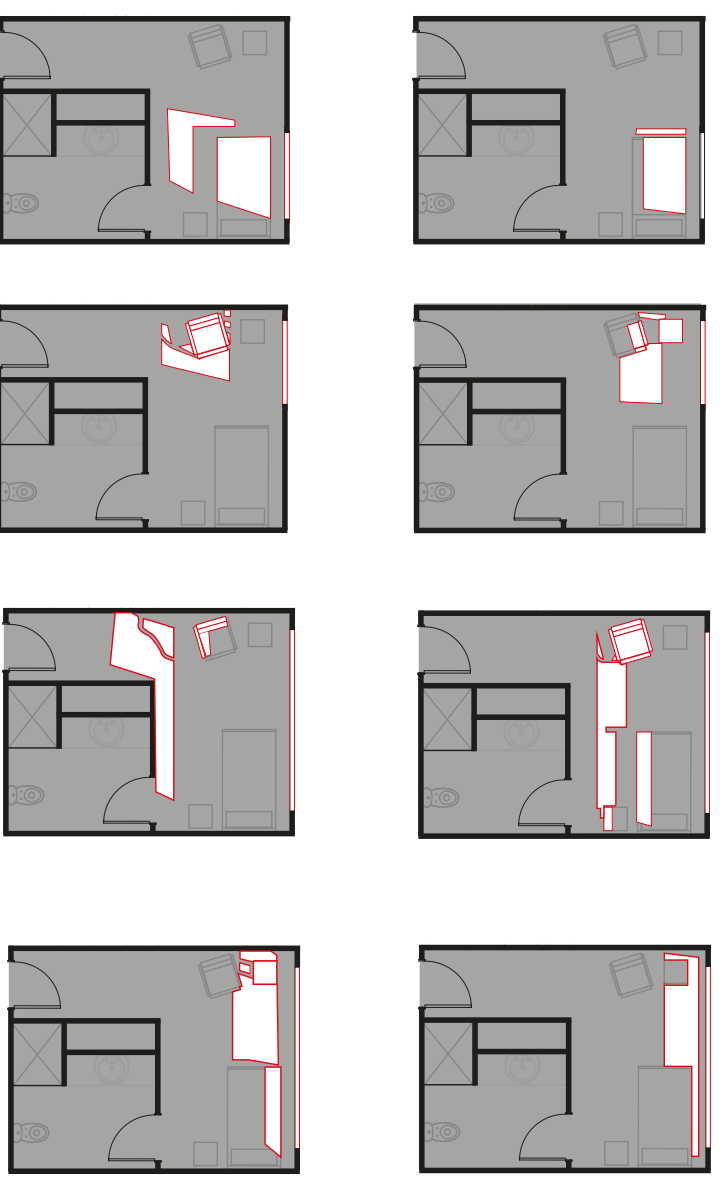
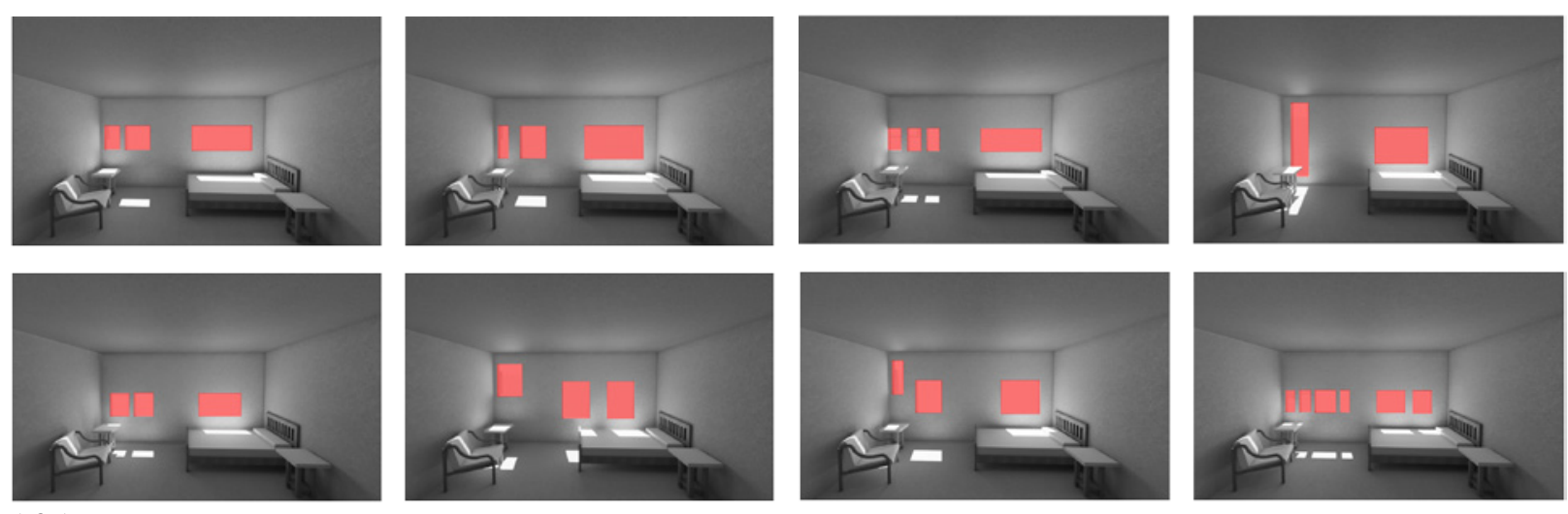


\section{The Corridor}

Upon leaving the bedroom, strategies to improve a resident's orientation and recognition of their room were tested through the use of colour and familial imagery (Figure 6.2.7). The offset alignment of bedrooms increased legibility within the corridor to allow improved priming strategies for residents as they wander (Figure 6.2.11). Furthermore, lighting strategies to provide increased stimulation and sense of movement for residents as they wander were tested through both artificial and natural light strategies (Figure 6.2.8 - 6.2.9). In order to reduce overstimulation with natural lighting, the use of skylights in combination with coloured walls was deemed the most appropriate balance in maintaining appropriate stimulation levels for residents (Figure 6.2.10).
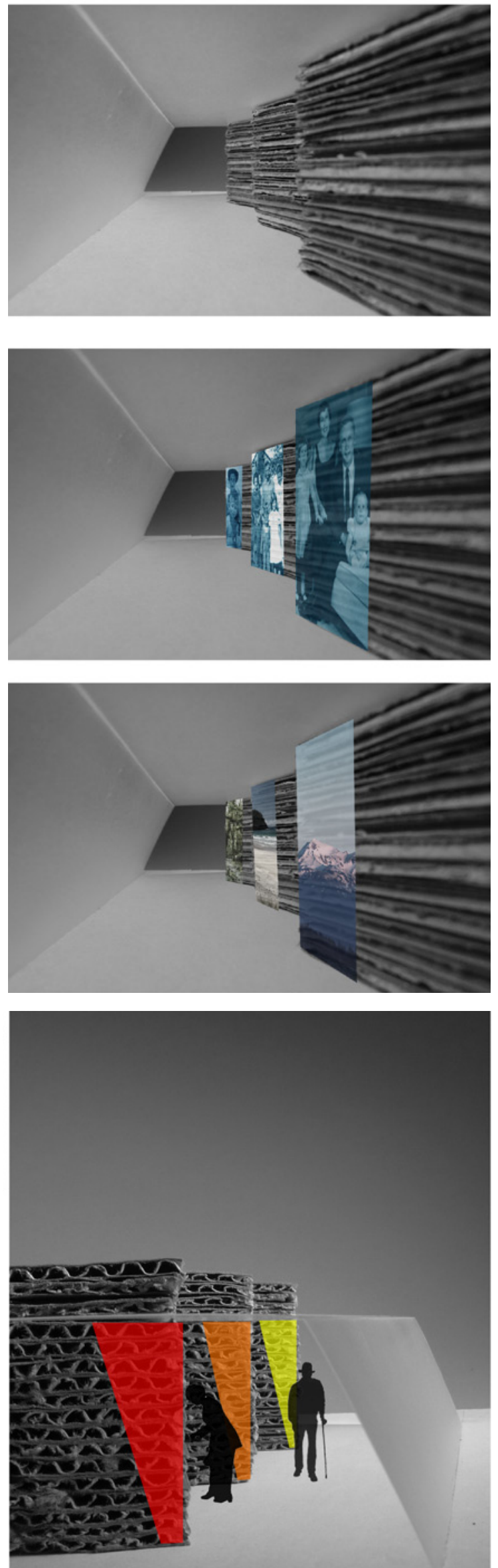

6.2 .7 

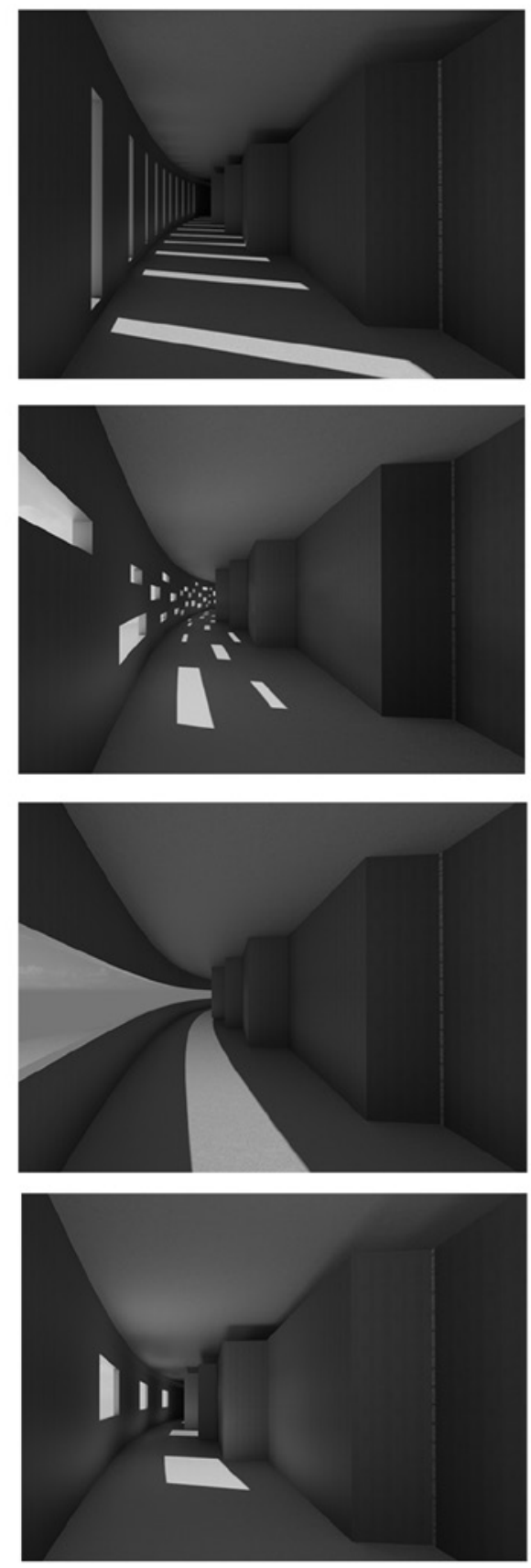

6.2 .8
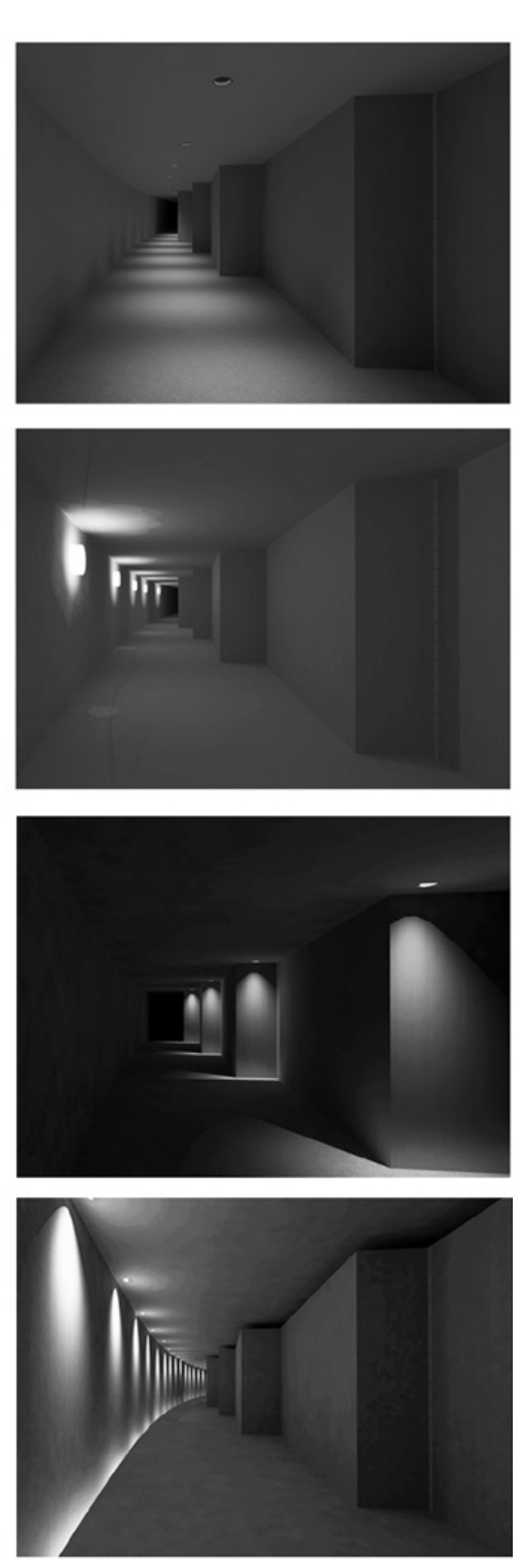

6.2 .9
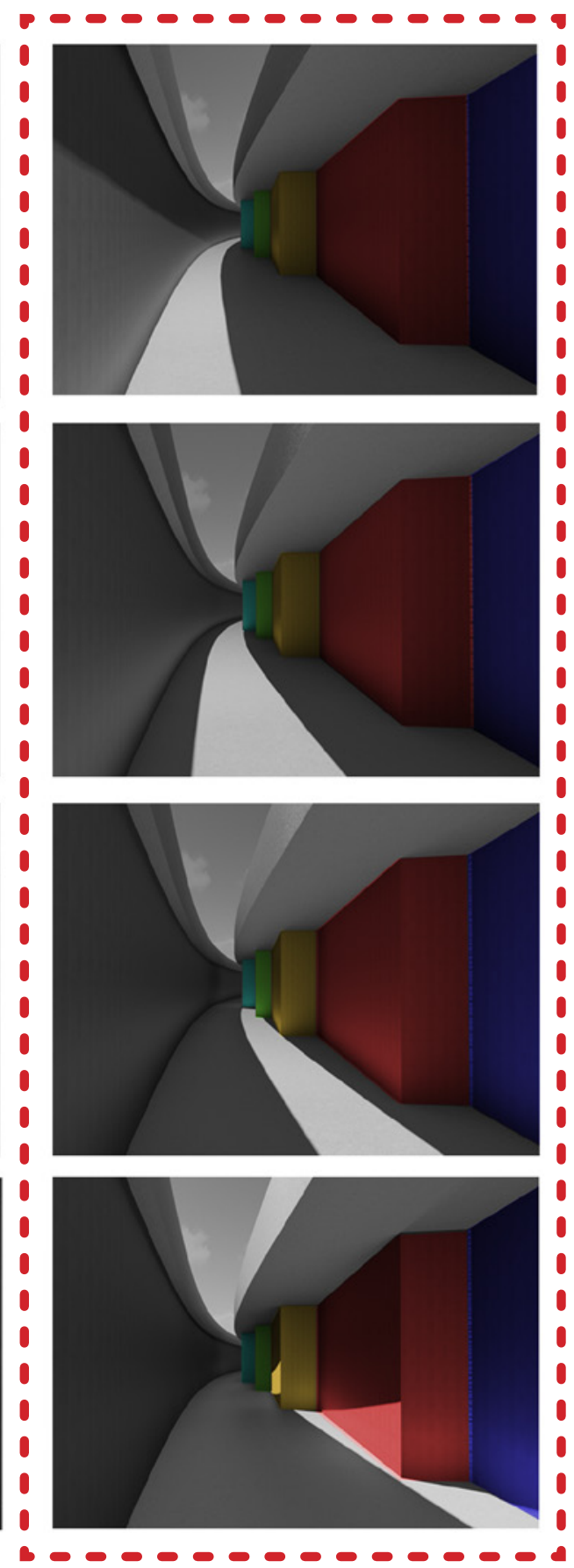

6.2 .10

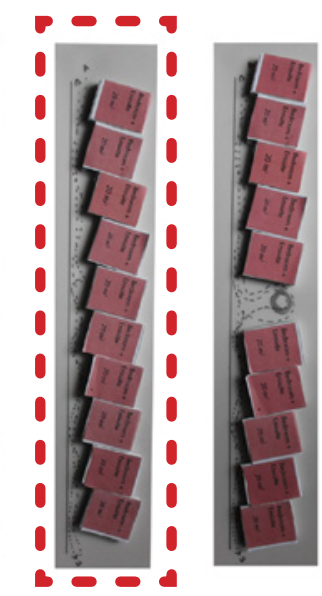

6.2 .11
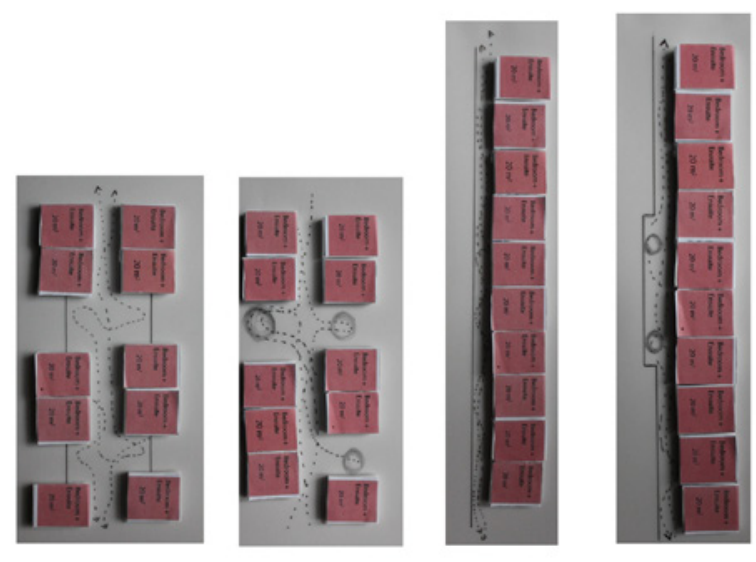


\section{Manipulating natural light}

To balance the need for morning light exposure and restricting light in the afternoon, levels were explored as a method of manipulating natural lighting requirements at these two moments in the day. Light wells tested opportunities for restricted light to promote movement, occupation and stimulation at specific times of the day on the ground floor (Figure 6.2.12).
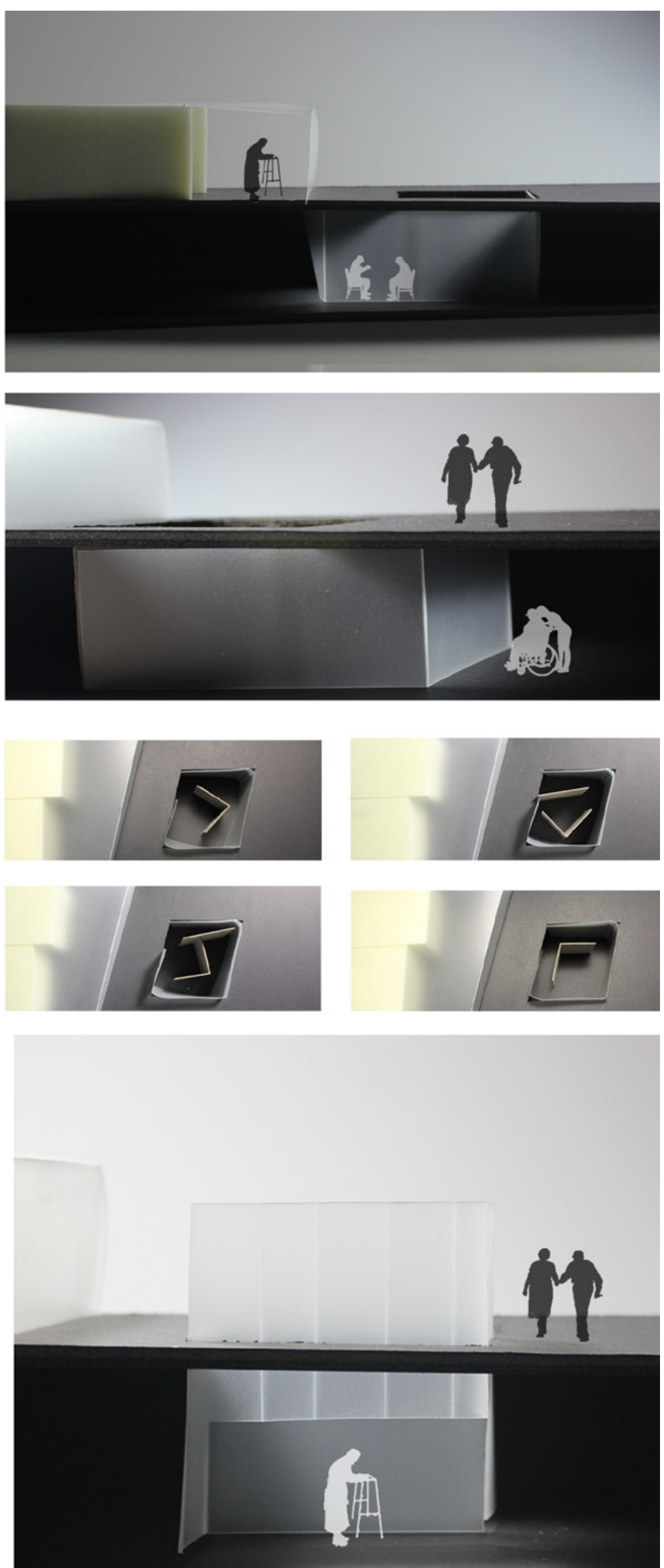

6.2 .12 

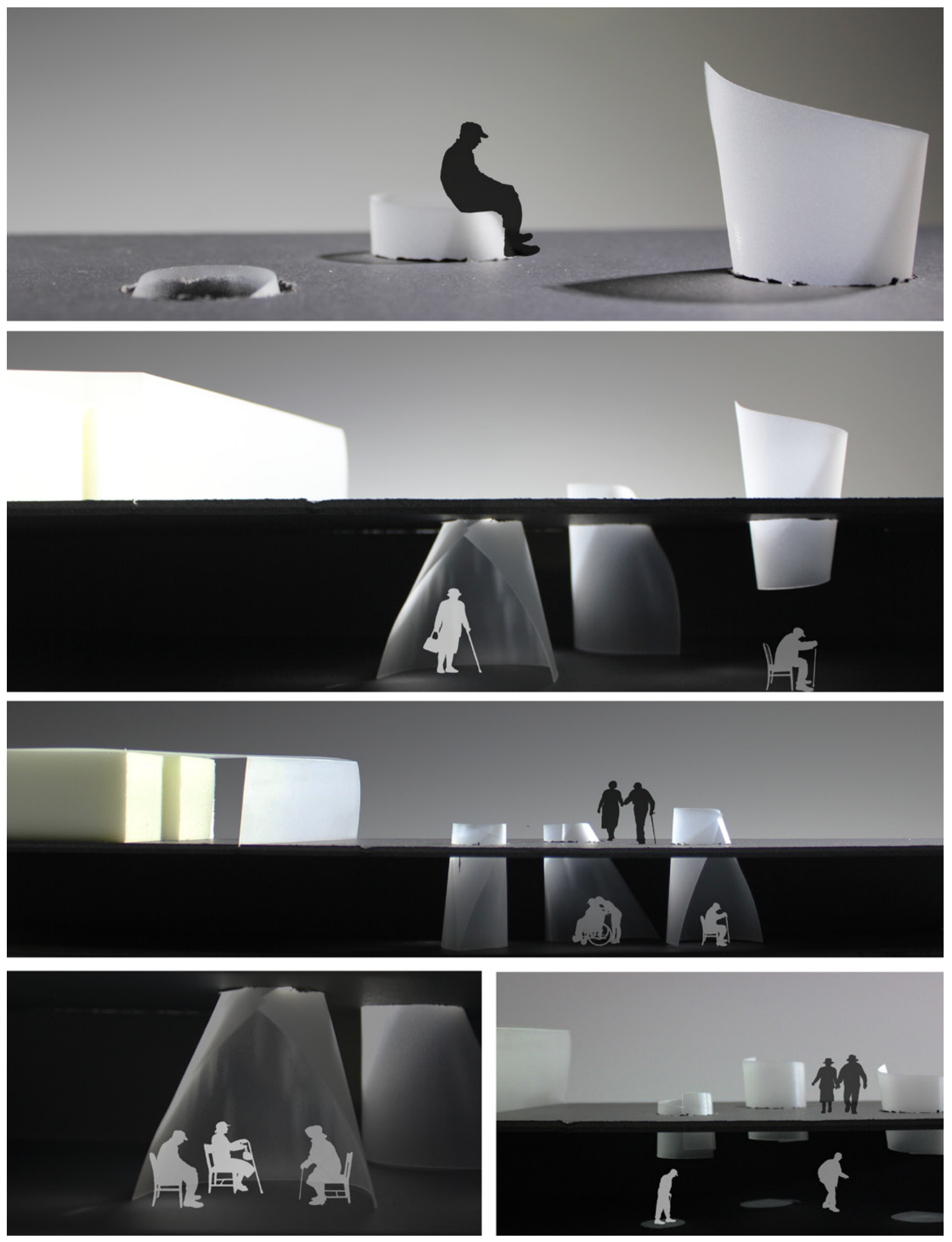


\section{Dementia Respite Development}

\section{Positives}

Spaces are dispersed between floors to allow for increased mobility for residents and control of natural light at different times of the day (Figure 6.2.12 \& 6.1.14)

Priming strategies through use of colour and imagery improve legibility for residents as they wander through building (Figure 6.2.13 \& 6.2.16)

Outdoor spaces alongside circulation routes allow for increased cognitive levels and natural light exposure for residents as they wander (Figure 6.2.15)

\section{Negatives}

Design approach is removed from constraints of site and integration with auxiliary programs, the integration of residents amongst the wider community is lacking.

The use of ramps to achieve inter-floor movement subsequently increased the desired mobility, with distances deemed inappropriate for residents who are either too frail or require wheelchairs.

\subsubsection{First floor plan}

6.2.13 First floor circulation perspective

6.2.14 Ground floor plan

6.2.15 Morning living room accessibility

6.2.16 Ground floor light well
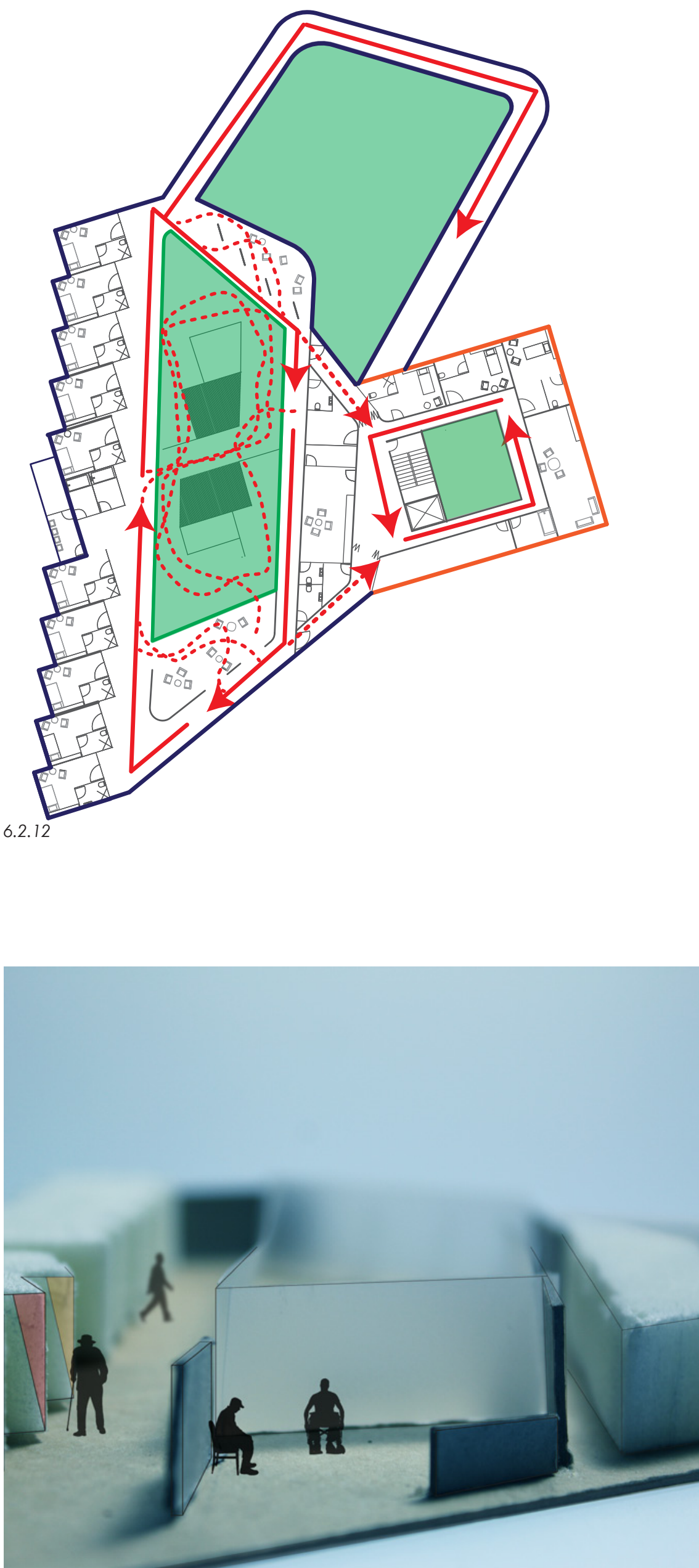

6.2 .13 

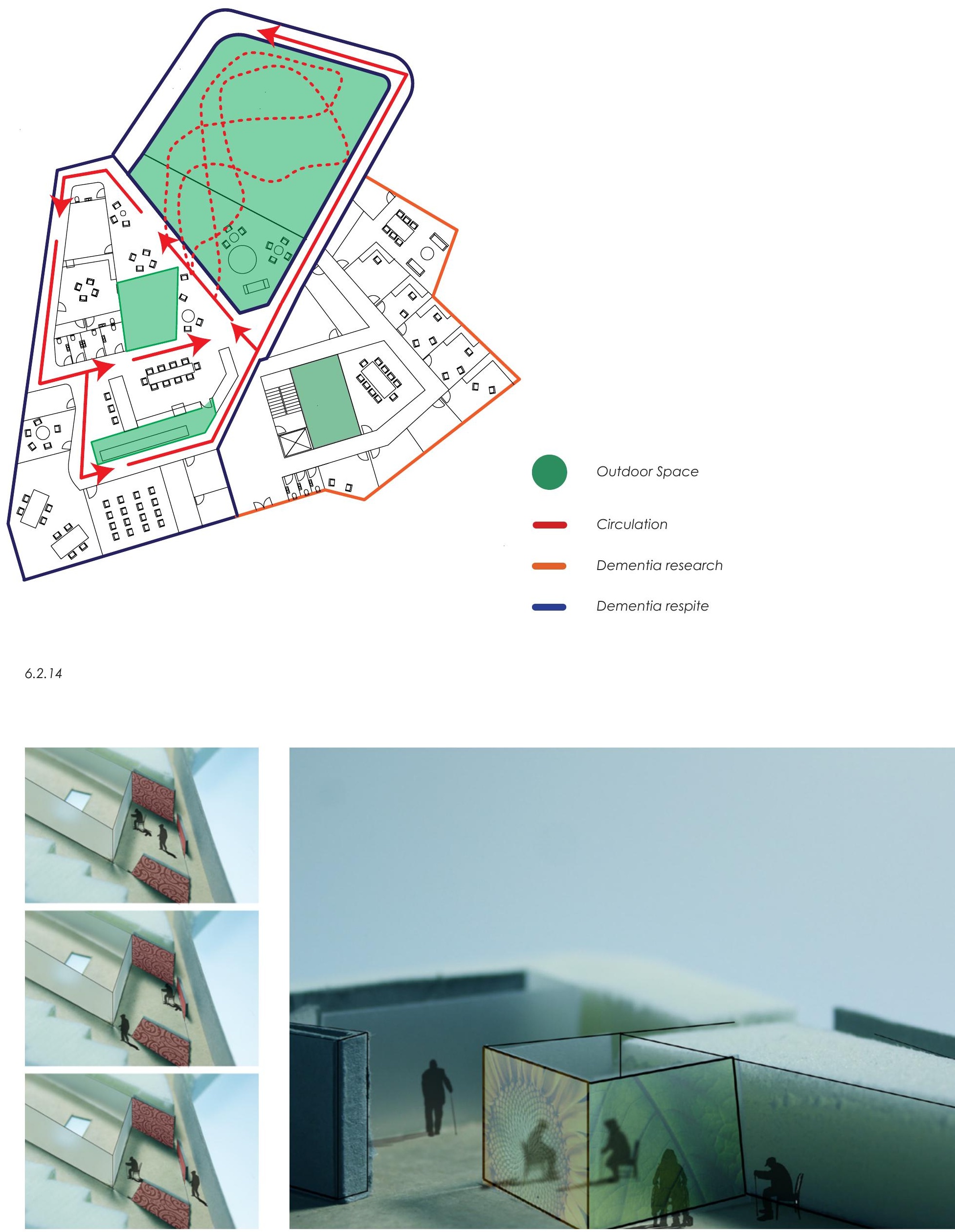
Design Phase One + "Inside - Out"

The success of the shared courtyard from design phase one was adopted within the "Inside - Out" design process to balance the design to cater for dementia residents. Massing studies tested building elements as priming strategies for residents as they wander through the building. Variations in roof profiles (Figure 6.2.17) and the separation of the corridor from building masses (Figure 6.2.18) helped to create building elements as points of interest along a continuous walkway. The elevated walkway allowed connections between building masses and maintained accessibility to the shared courtyard for the medical community below.
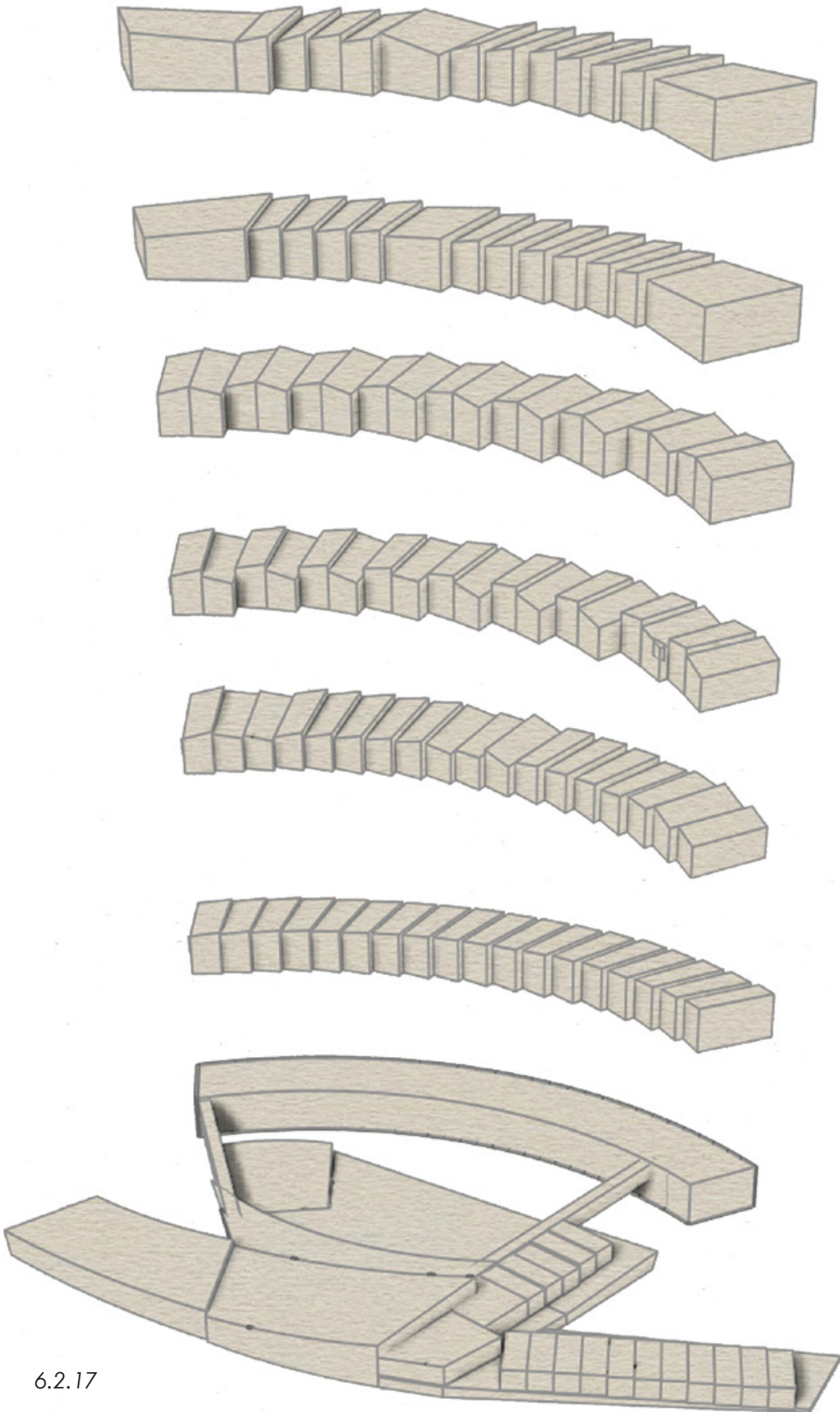

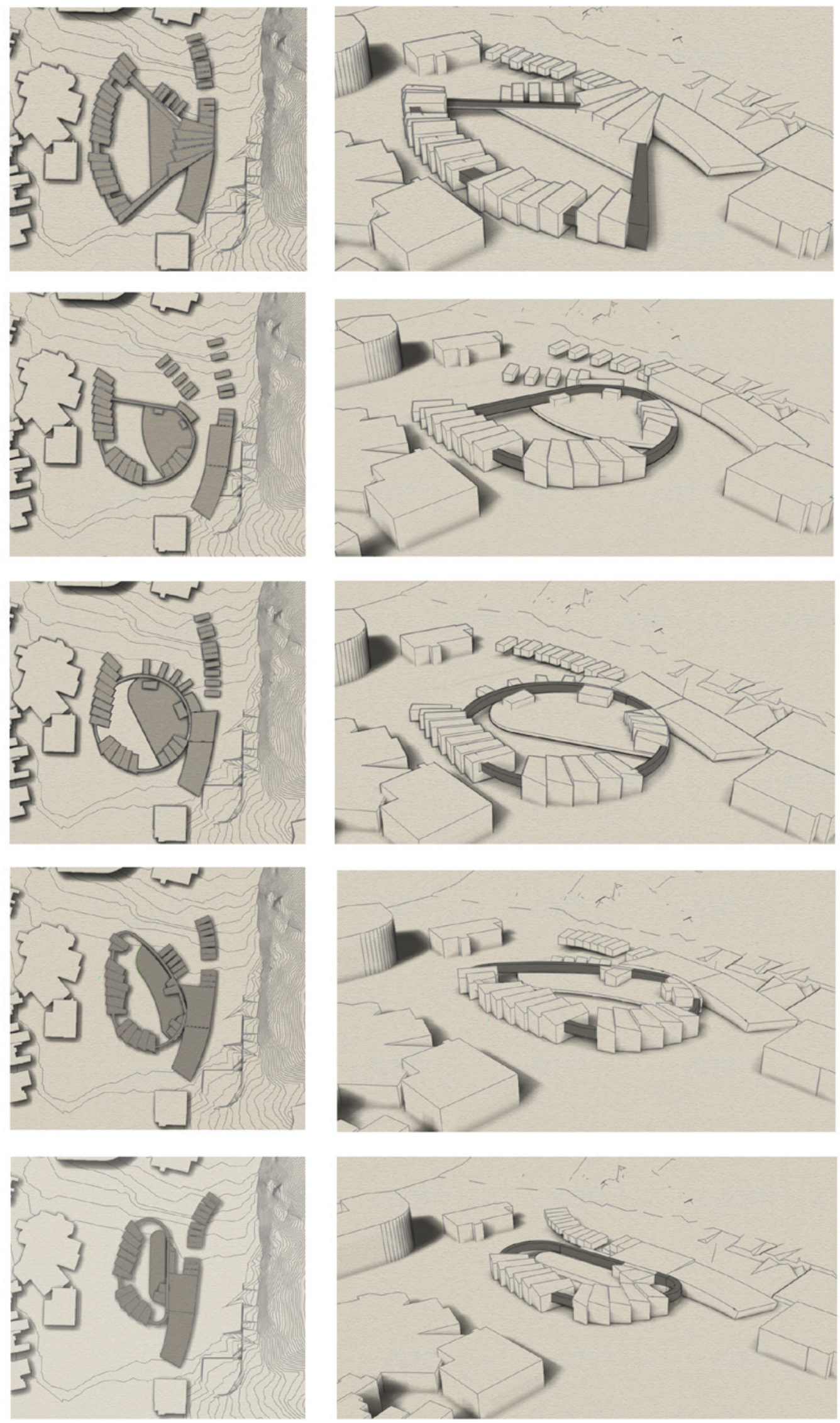

6.2.18 
Spatial Arrangement

(Light + Movement)

Spaces were positioned along the continuous walkway to facilitate movement of residents according to the circadian rhythm of the sun. Adopting Knowles's theory of the interstitium, spaces were orientated in different directions to achieve variation in light exposure at different times of the day (Figure 6.2.20). The spaces that appear to extrude from the walkway (Figure 6.2.19) are also used as environmental indicators to help assist orientation for residents as they wander along the continuous walkway (Figure 6.2.21).
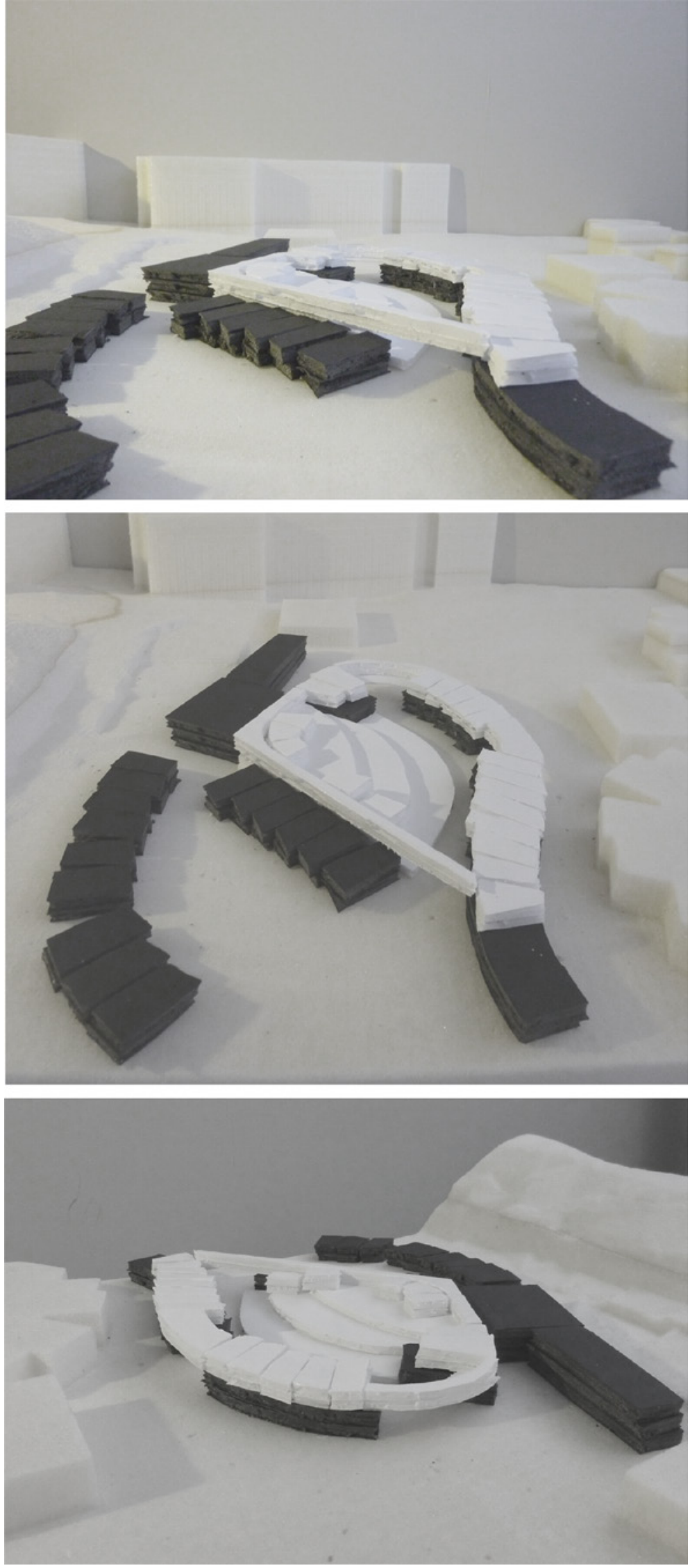

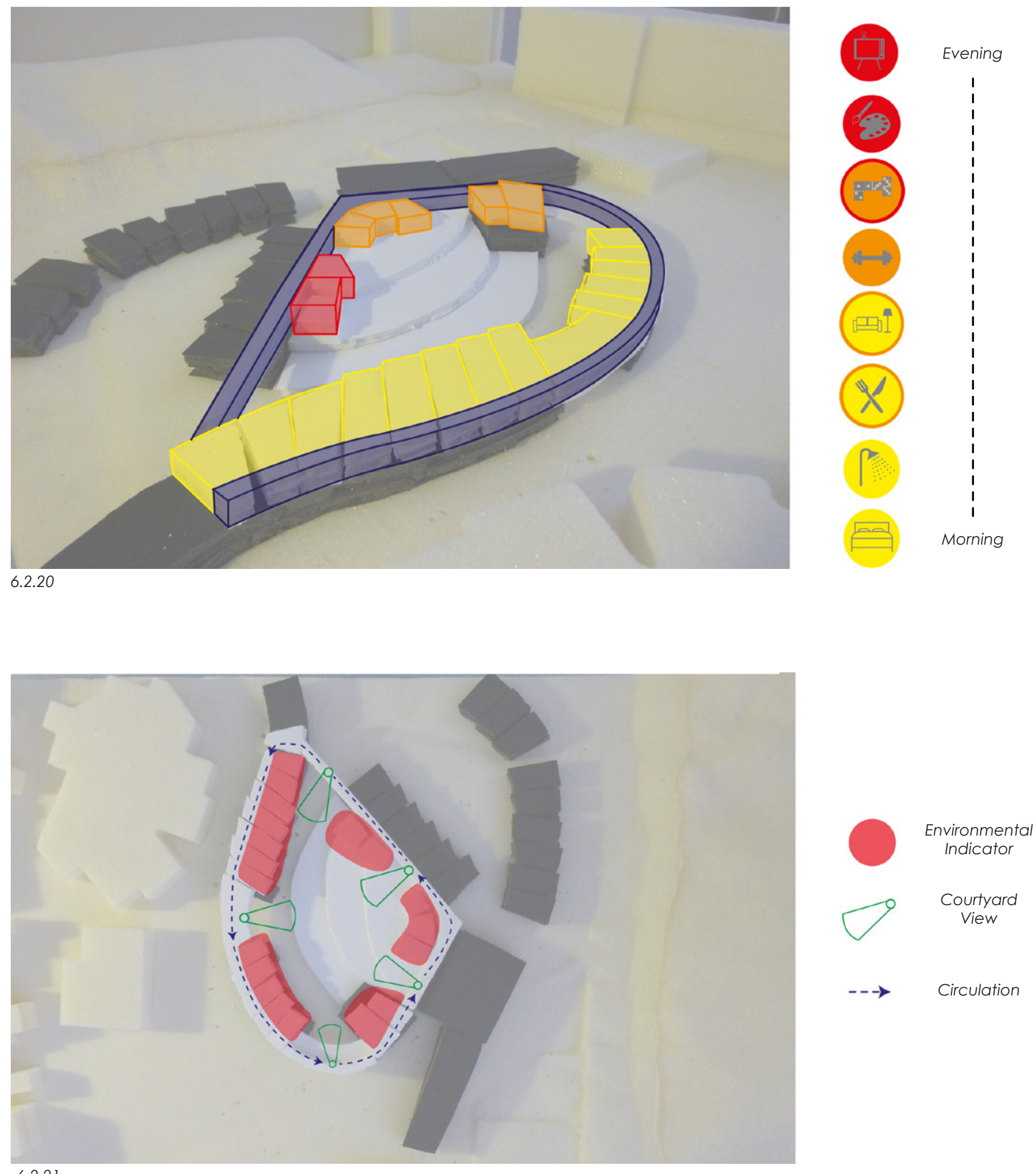
To improve these spaces as environmental indicators within the courtyard, variations in cladding, colour and window form were tested to improve the legibility of a resident's bedroom while wandering (Figure 6.2.22). A series of drawings were also used to explore domestic profiles that would help create a familial and legible environment for residents within the courtyard (Figure 6.2.23).
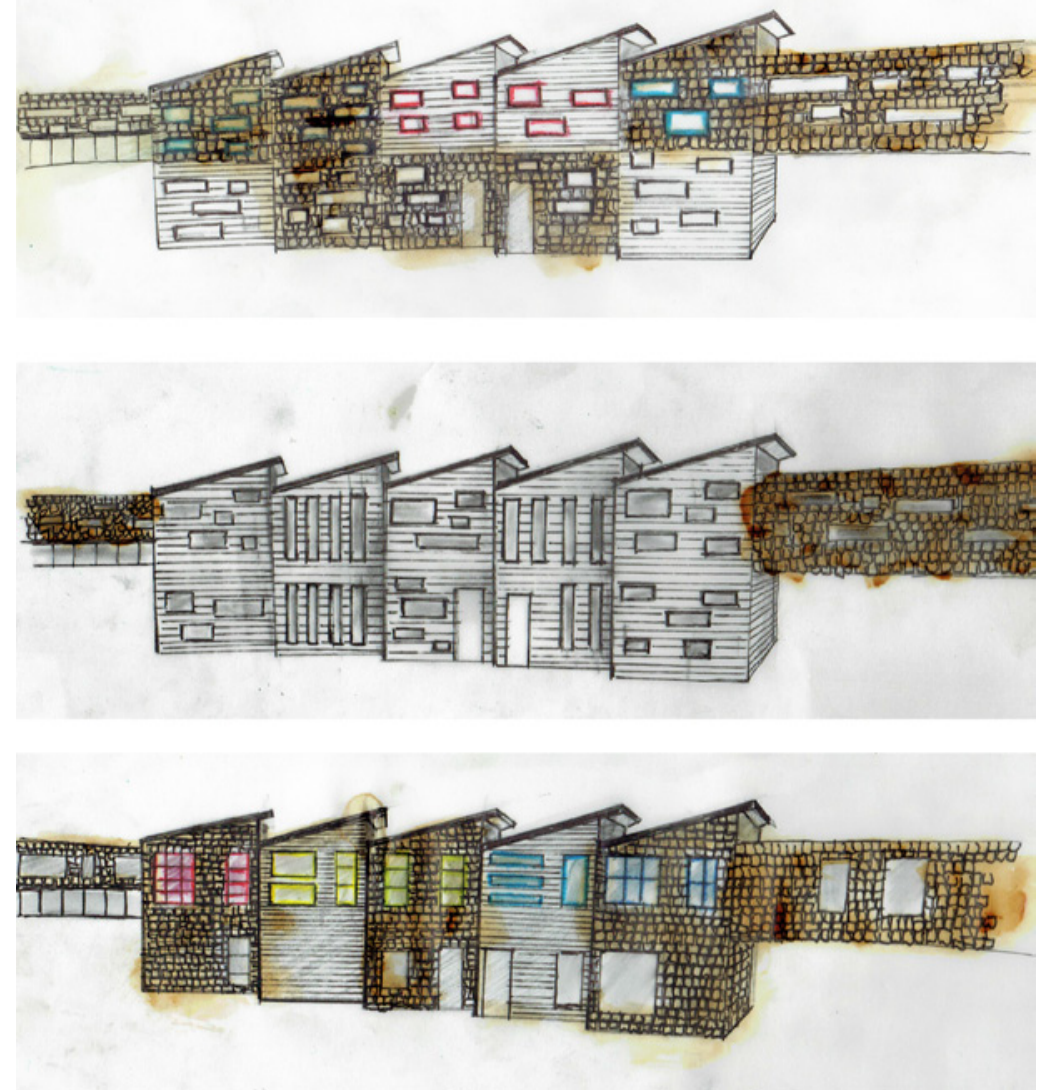

6.2 .22 

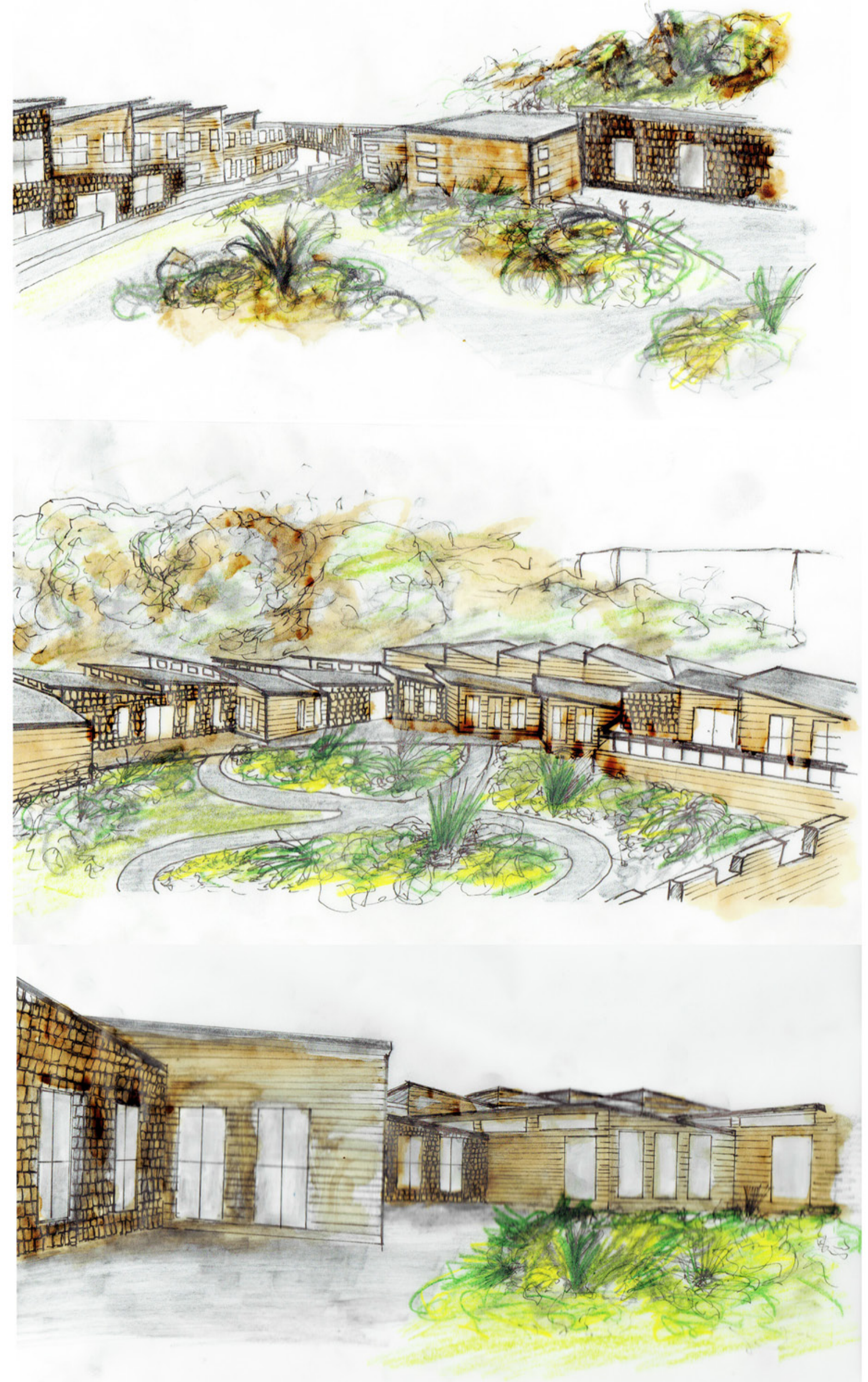


\section{Exterior Aesthetic}

The treatment of the exterior form is in contrast to the domestic aesthetic of the interior courtyard, reflecting a modern aesthetic that integrates within its surroundings (Figure 6.2.25). Emphasizing the curved form generated by the circulation path of dementia residents above, the simple exterior profile expresses this movement through simple material gestures. The contrast in timber cladding on the first floor with glass panes below gives a visual appearance of the mass floating above the ground. Variations in the spacing of timber louvers on the ground floor help emphasize the curves of the building to add to this sense of movement, drawing interest and movement of the medical community towards the building (Figure 6.2.24).
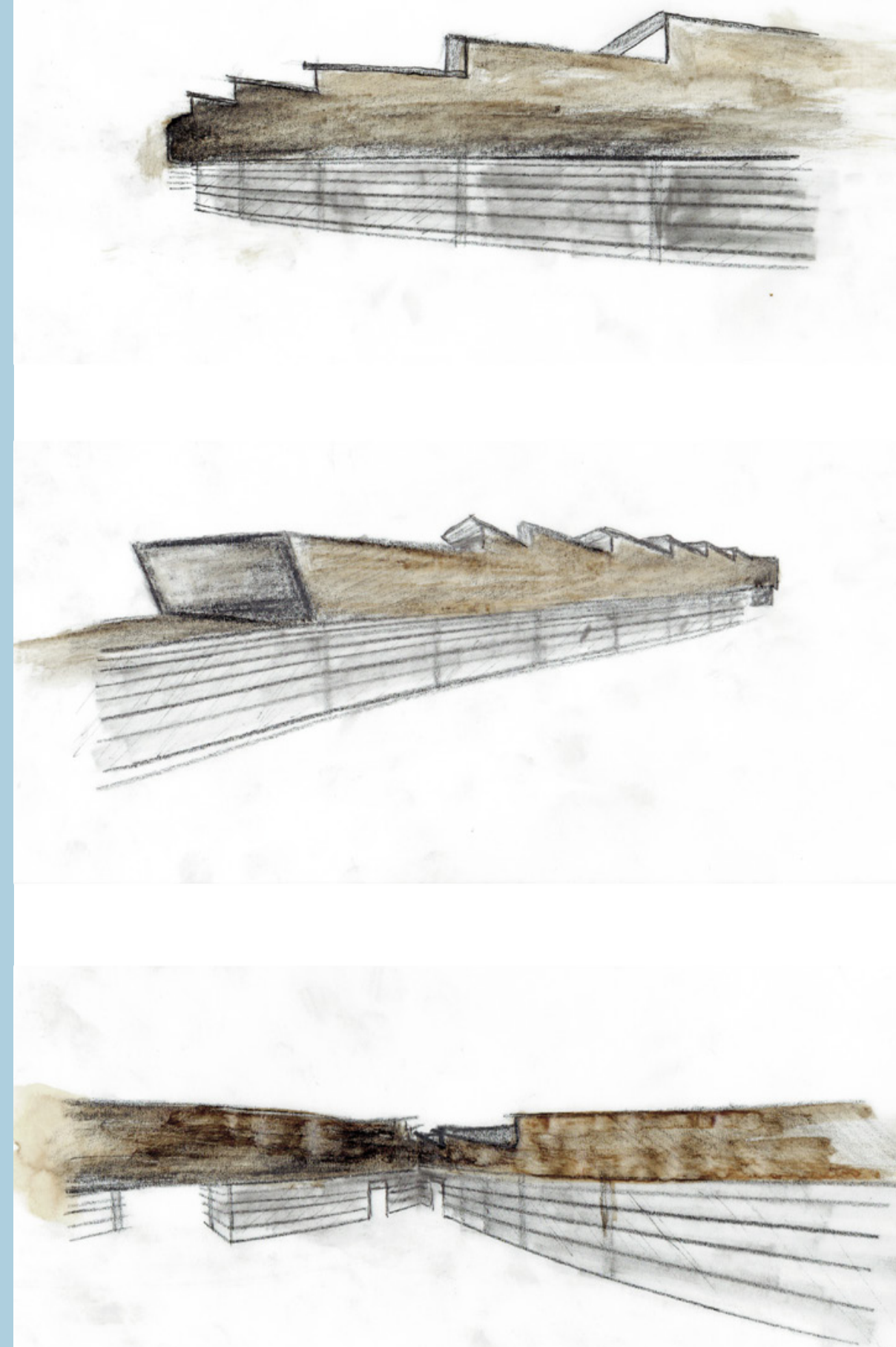

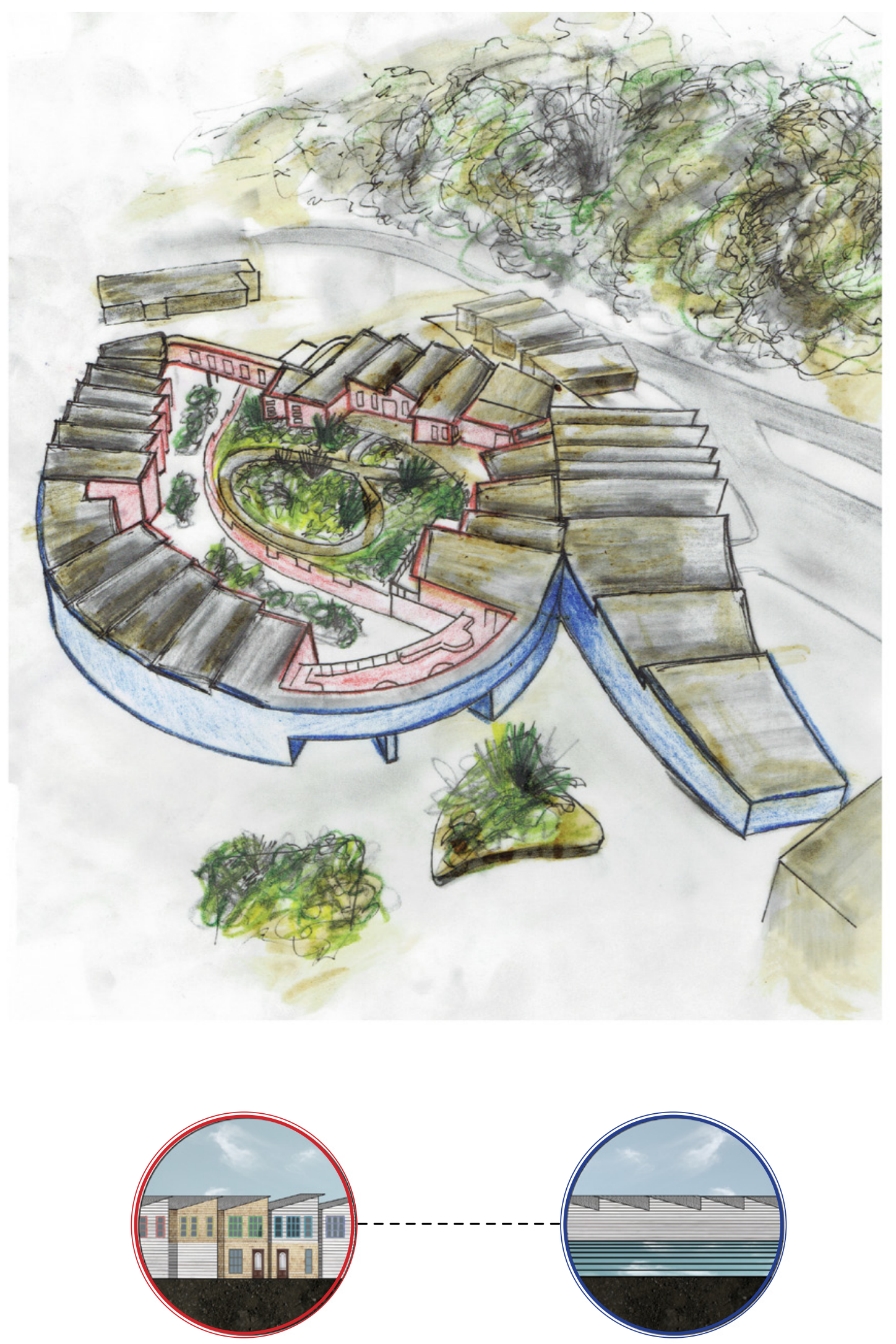

6.2.25 


\section{Concept Development}

\section{Strengths}

The contrast in aesthetic between the domestic courtyard and institutional exterior balances the needs of the different occupants who interact with the facility

Timber clad exterior walls on the first floor maintain privacy for residents and direct orientation and interest towards the internal courtyard (Figure 6.2.29)

Internal courtyard promotes a social hub among residents and the medical community to interact within a familial domestic setting (Figure 6.2.27)

\section{Weaknesses}

The buildings reliance upon natural light for stimulation and circadian alignment limits its ability to cater for more severe disrupted sleep cycles as the external environment for stimulation and therapy becomes less effective as dementia progresses.
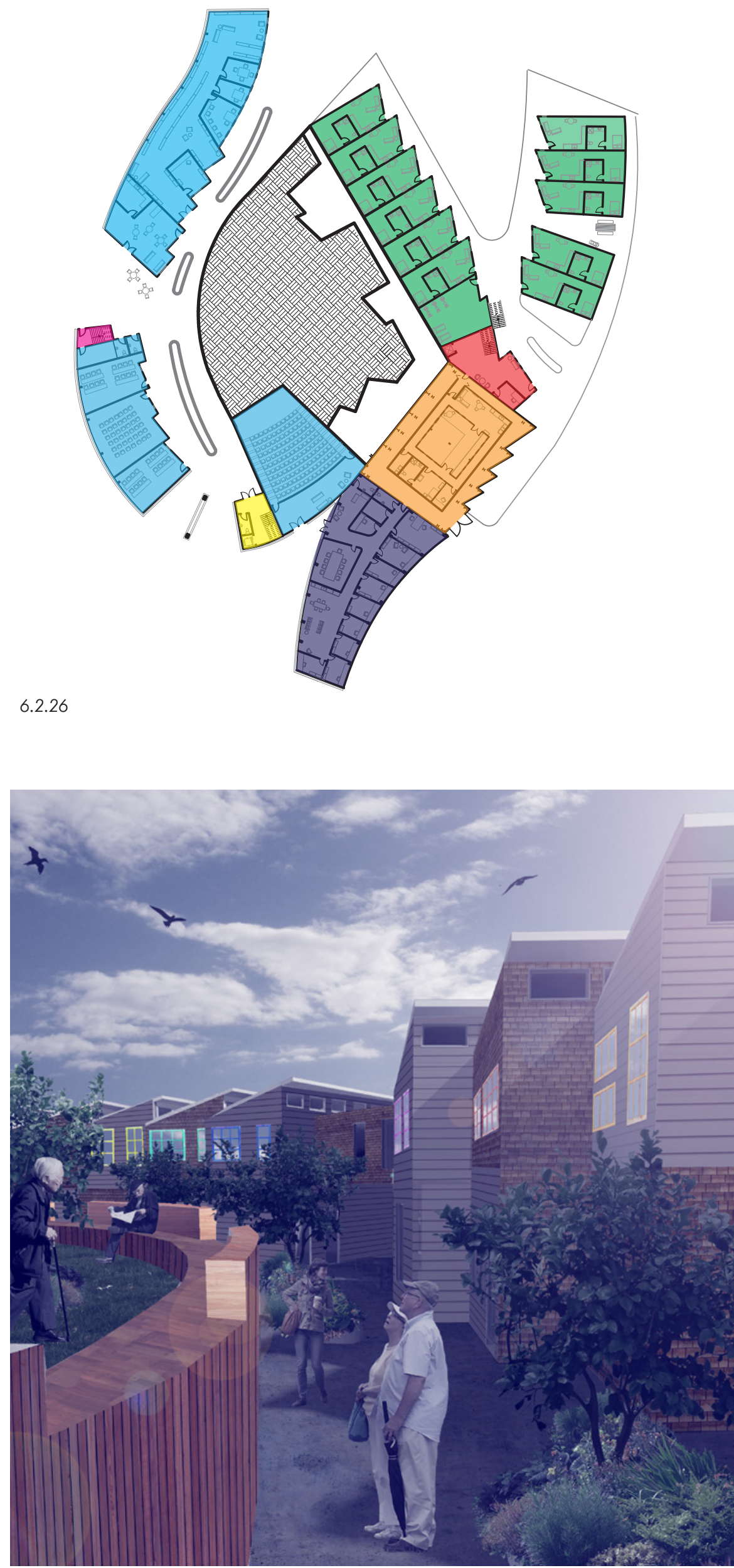


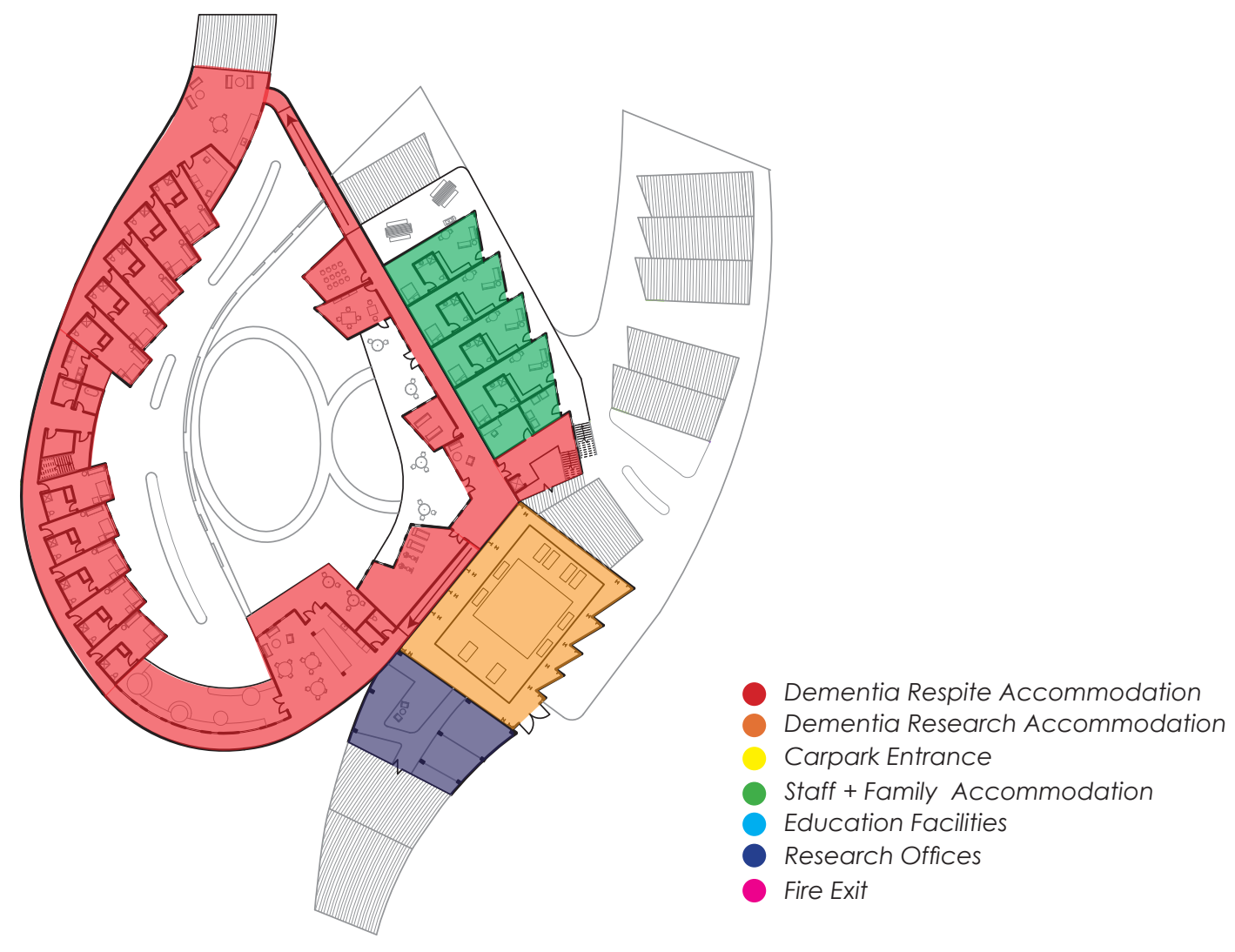

6.2.28

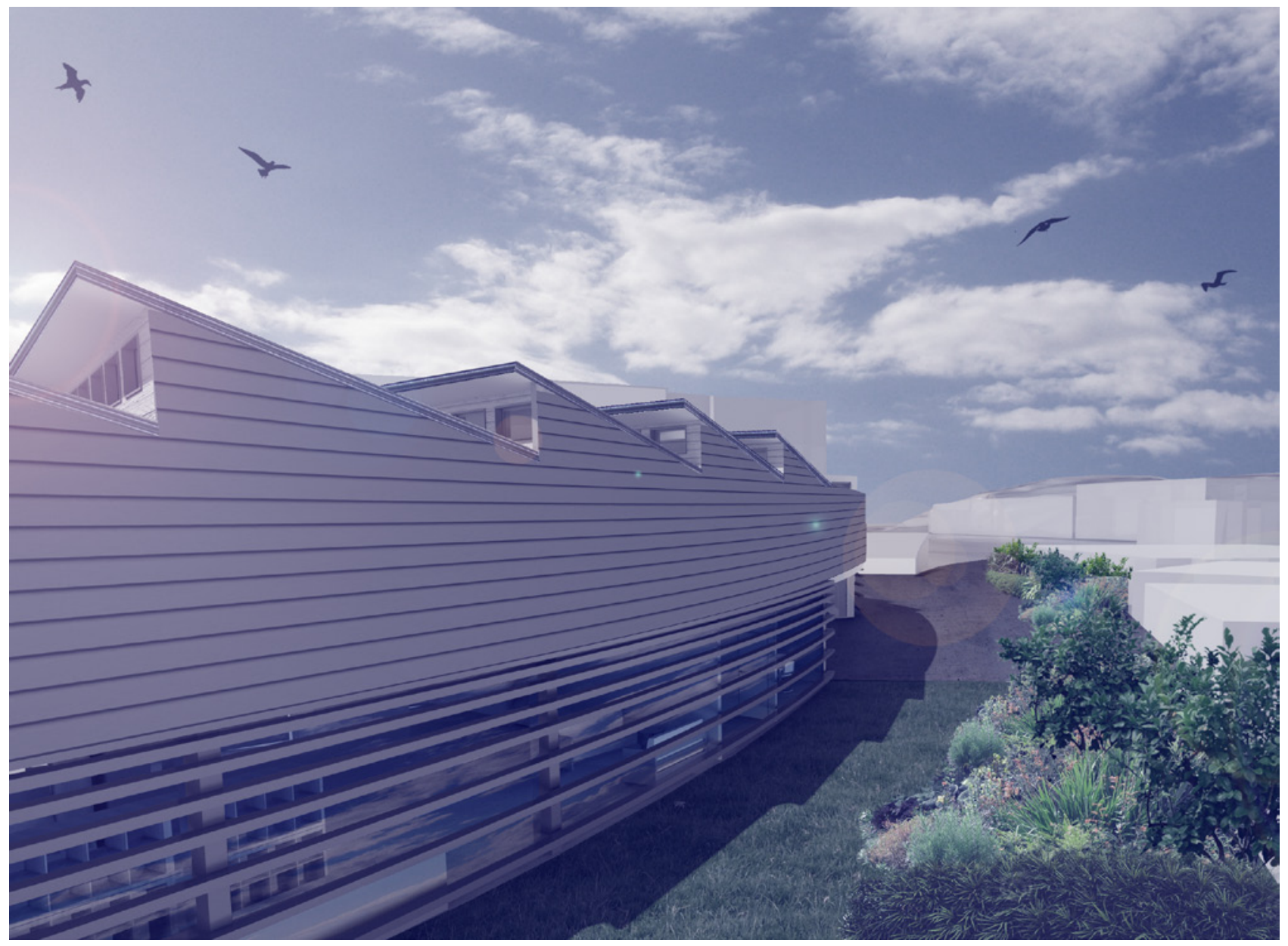




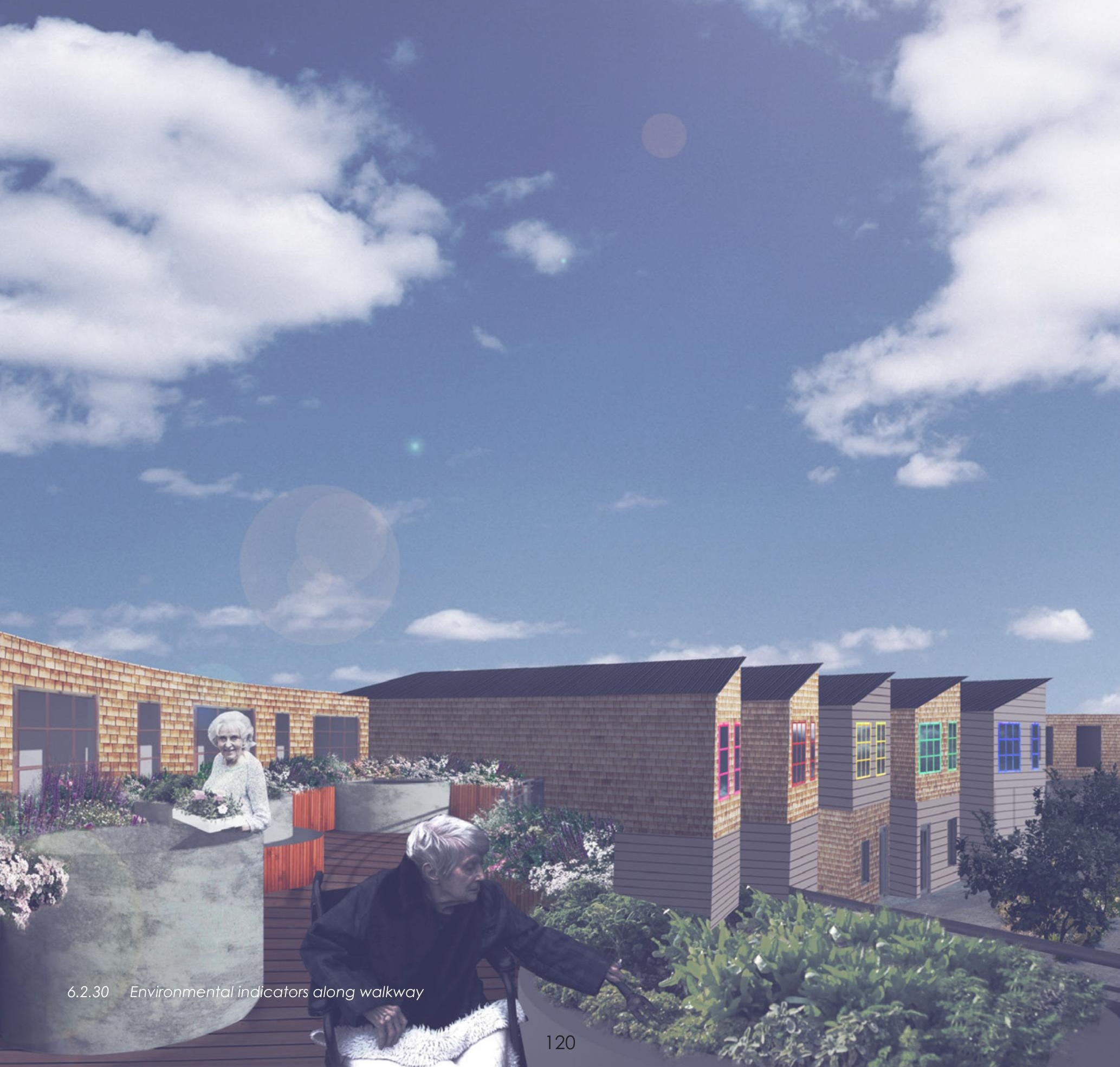




\section{Reflection + Summary}

Adopting an inside-out approach to the design process provided a valuable insight towards developing design methods to assist in the wellbeing of dementia residents. Although initial tests were not restricted by form or site, they helped develop movement and light objectives towards the desired sensations and experiences of the dementia resident while in respite care. Integrating these findings alongside design phase one allowed for a more robust design that balanced the needs of both the medical community and dementia residents. However, the design process also illustrated the limitation of the relationship between architecture and the external environment to initiate circadian rhythms. As symptoms of dementia progress, the influence of the external environment to provide the necessary stimulation to influence disruptive sleep behaviour diminishes. Further explorations towards the potential for artificial lighting environments to reduce the limitations of the external environment for sleep therapy begins the design process of design phase three.

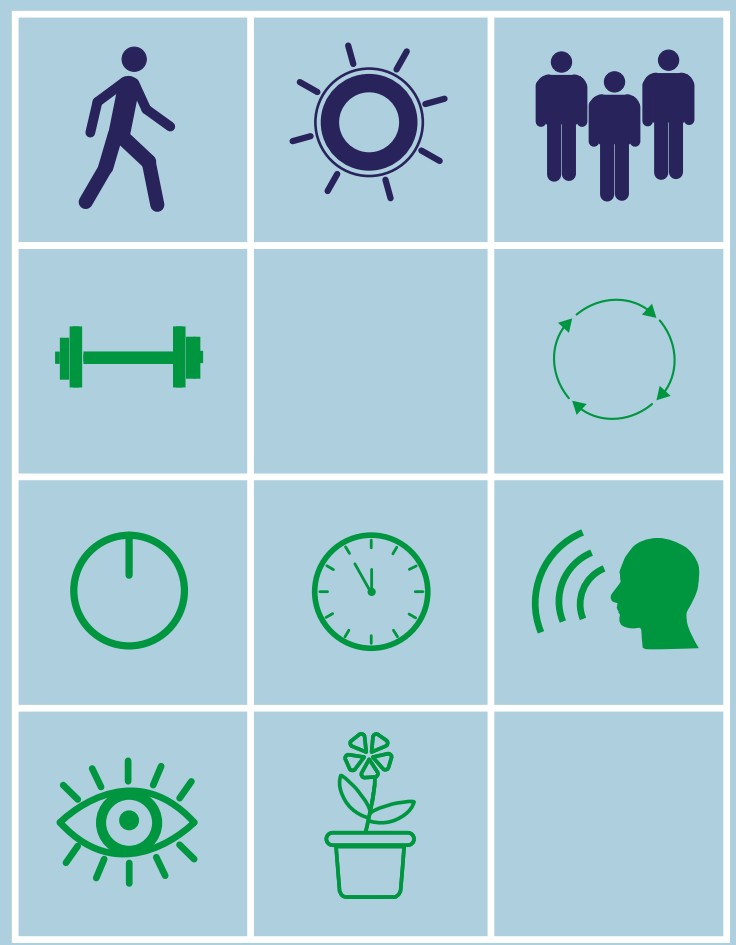




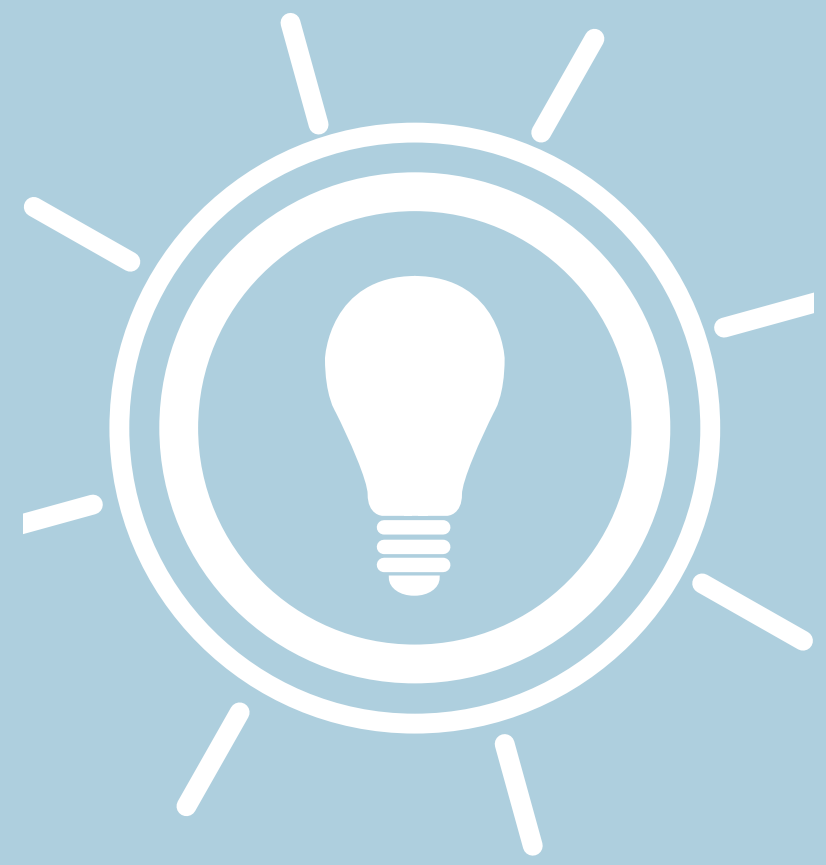




\section{3}

\section{Design Phase 3 \\ "Simulation"}

"It is no longer a question of imitation, nor duplication, nor even parody. It is a question of substituting the signs of the real for the real." (Baudrillard 2)

As dementia symptoms progress, the influence of natural light to regulate sleeping behaviour becomes less effective as residents become increasingly detached from natural stimuli. The following chapter builds upon current sleep therapy practice that was identified in the Massey University Sleep/Wake Research Centre, providing an alternative response to artificial lighting environments that simulates the natural to benefit the well-being for those affected by dementia. 


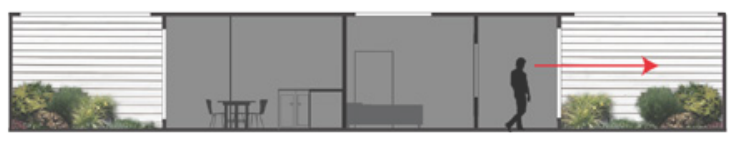

Perimeter

\section{Artificial Courtyard}

The courtyard typology was considered a key strategy towards simulating a familiar domestic outdoor space within the sleep lab. Various courtyard layouts were tested to promote movement and the use of artificial outdoor spaces within a confined $170 \mathrm{~m}^{2}$ footprint (Figure 6.3.2). The central courtyard provided the best balance between these two design imperatives that helped to reduce feelings of confinement for dementia patients within the sleep lab, focusing the resident's orientation towards the centre of the lab (Figure 6.3.1).

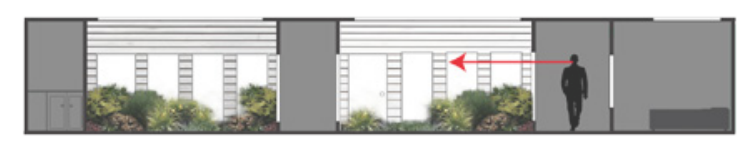

Multiple

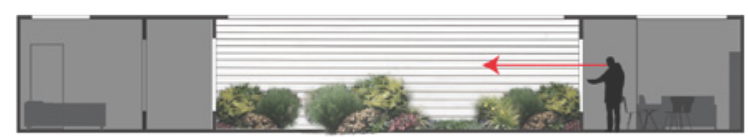

Central 

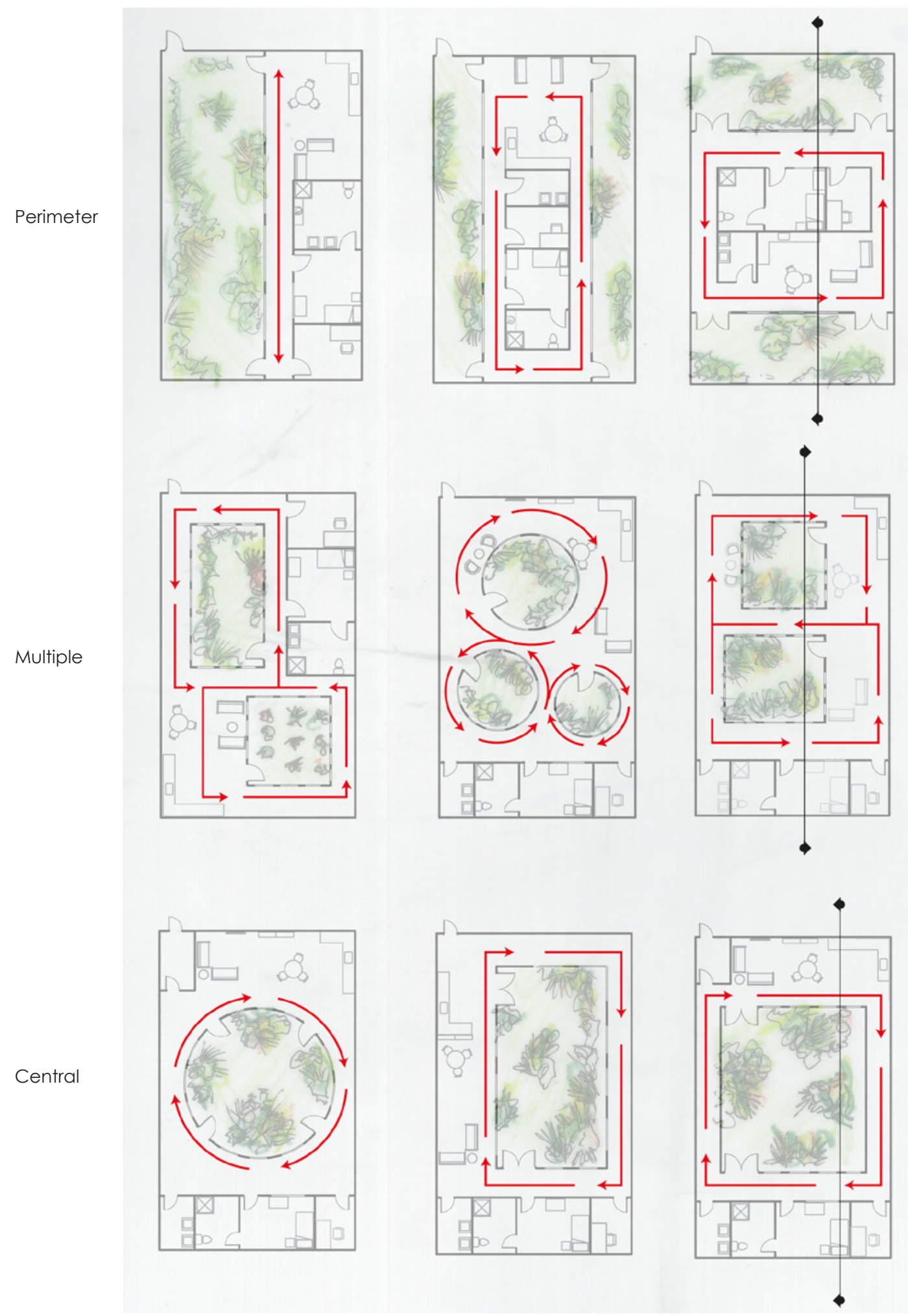

6.3.2 


\section{Artificial Outdoor Light}

Current methods of artificial light therapy were reimagined towards simulating a connection to the outdoors. Various domestic roof profiles and skylights (Figure 6.3.3 - 6.3.4) were tested to introduce familiar natural lighting strategies within this artificially lit environment. The form, scale and hierarchy of skylights emphasized visual orientation towards the central courtyard space. The use of exterior cladding within the courtyard further reinforced this notion of an outdoor environment, testing variations of timber and brick cladding styles (Figure 6.3.5).
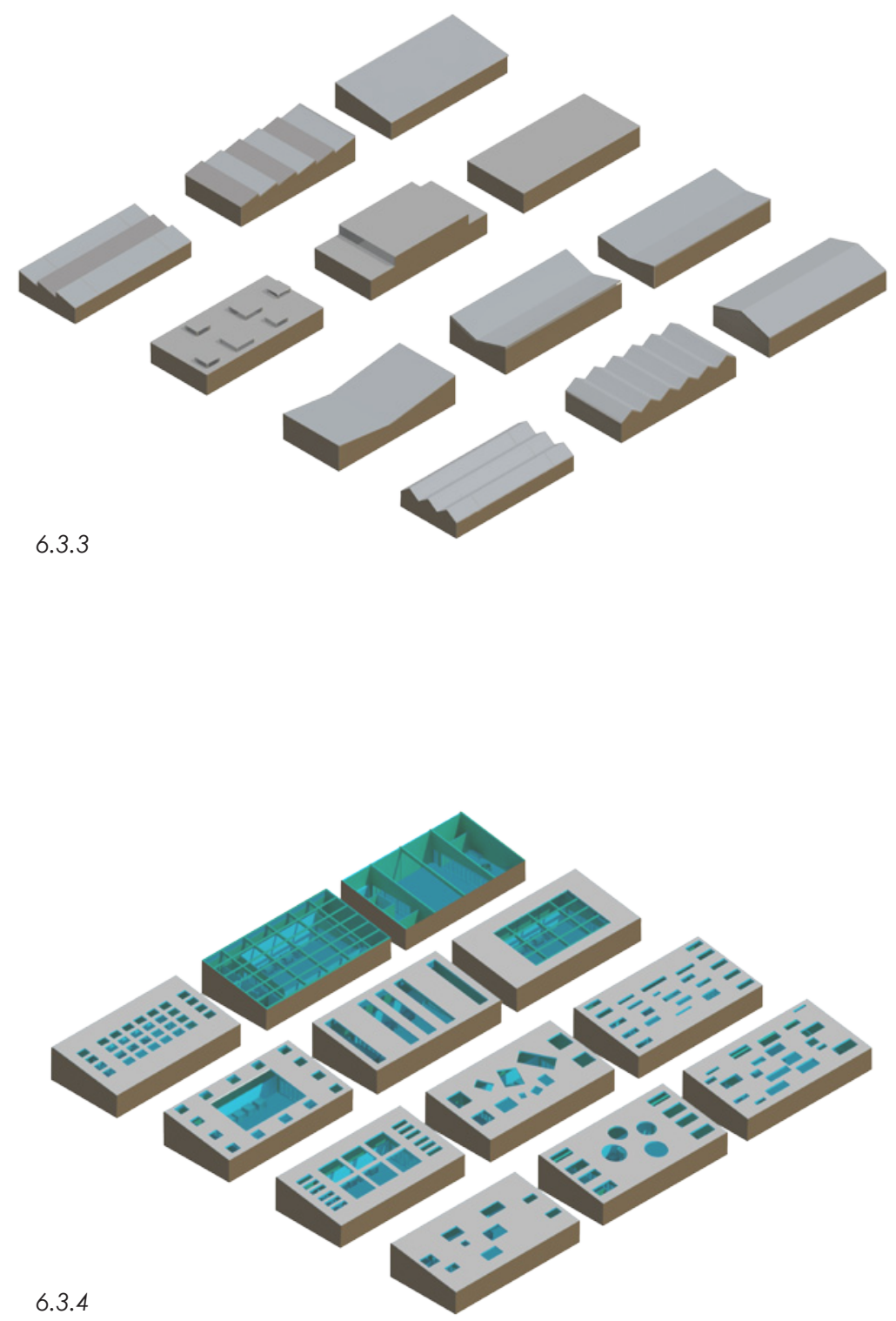

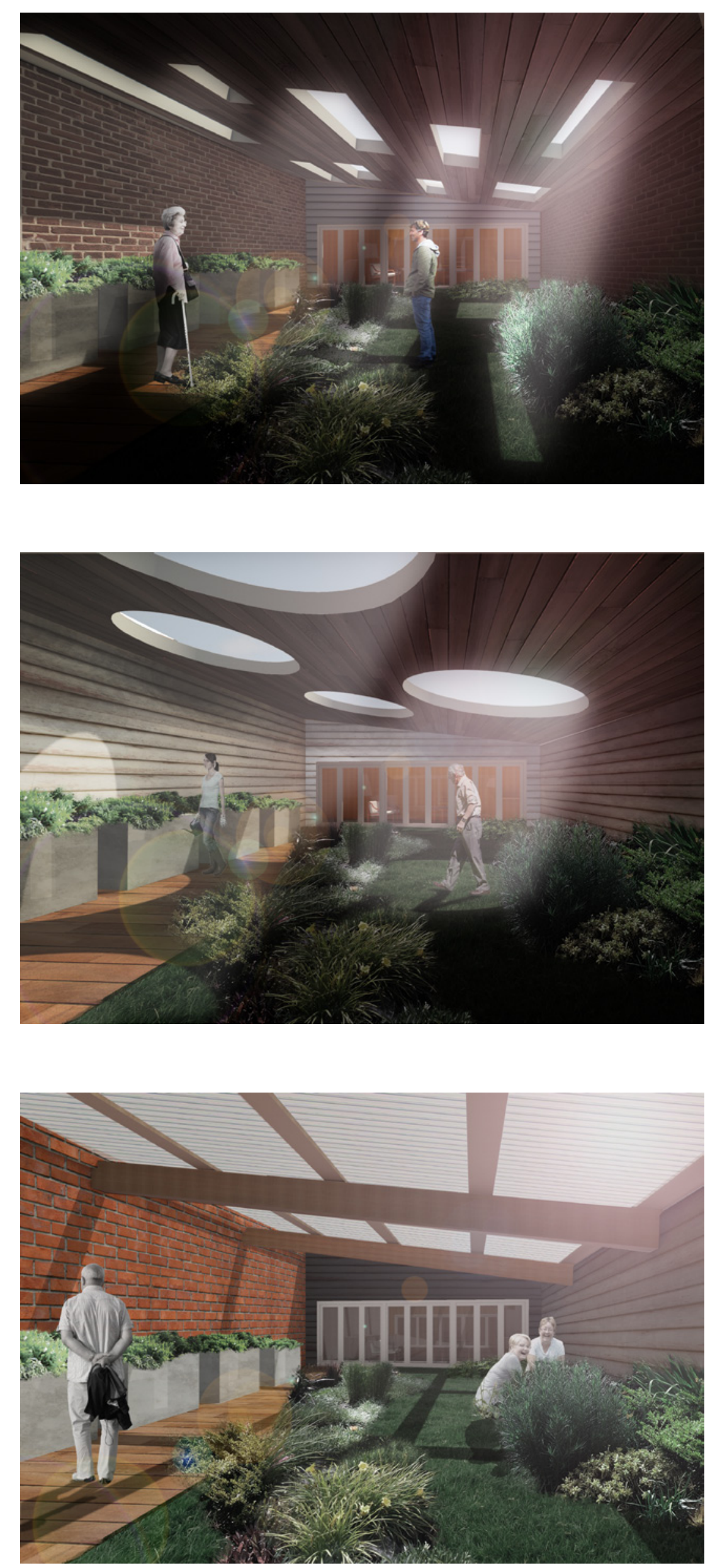


\section{Flexible system for imitation}

Development of the room within a room layout introduced key design ideas to allow for improved flexibility for a more person centered approach towards the design of the sleep lab (Figure 6.3.6)

\section{Mechanical Services}

Integrating an overhanging crane within the lab allows for improved assembly and efficiency in the construction process (Figure 6.3.7 - 6.3.8), testing variations of the sleep lab to cater to specific individuals or groups.

\section{Lighting}

Removing artificial light panels in preference for stage lighting system was adopted to imitate natural light qualities within interior and allow flexibility to roof profile

Fresnel lantern was deemed most appropriate in imitating natural light qualities with soft light edges and providing wash light over large surface area.

Spotlights were utilized to allow increase in light intensity and allow colour variation within interior space.

\section{Acoustics}

Walls lined with fire resistant acoustic foam panels to reduce construction noise for surrounding parts of the building.

\section{Large Openings}

Large door openings allow vehicular access to transport materials and prefabricated modules to increase efficiency of construction
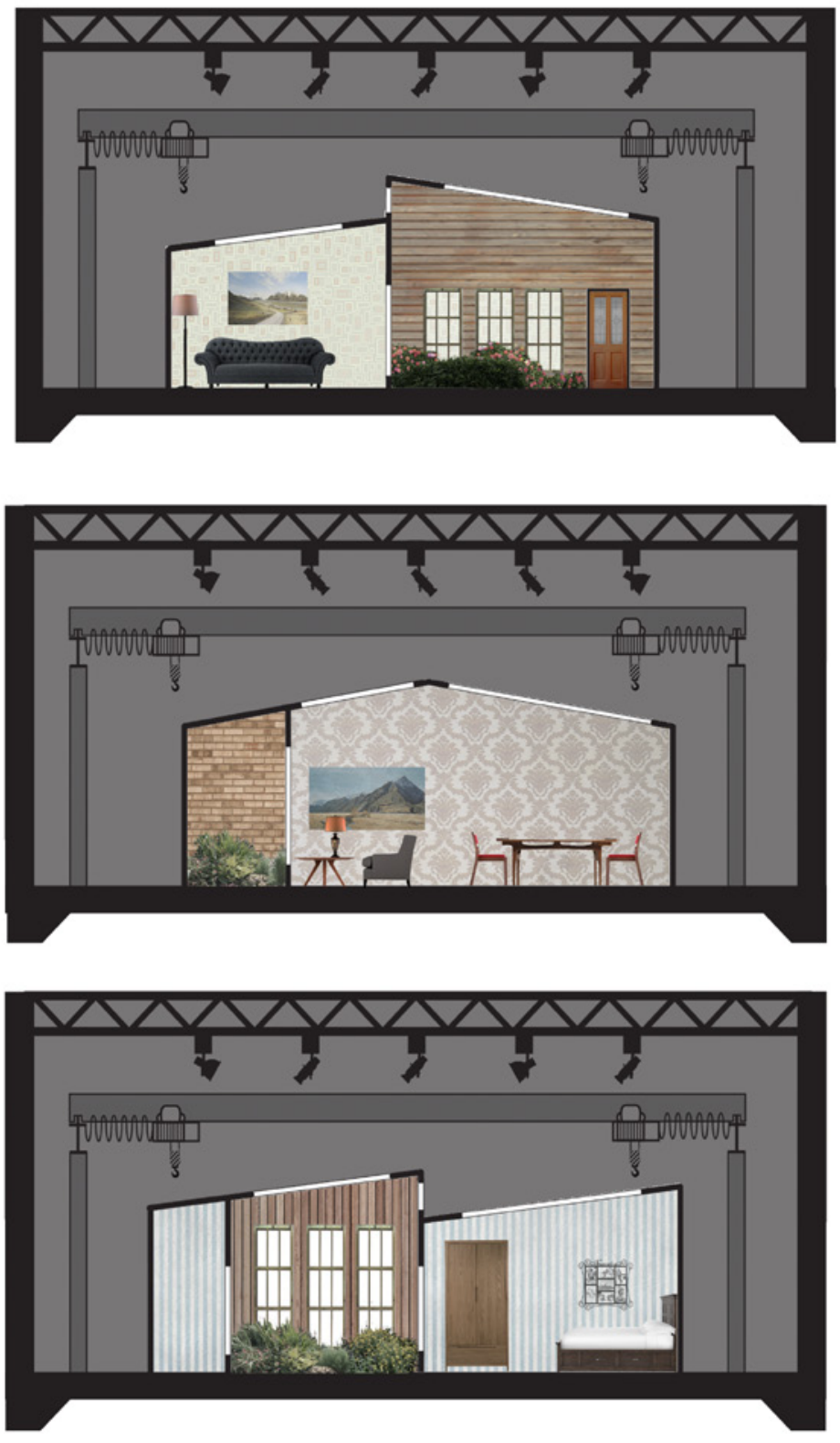

6.3.6
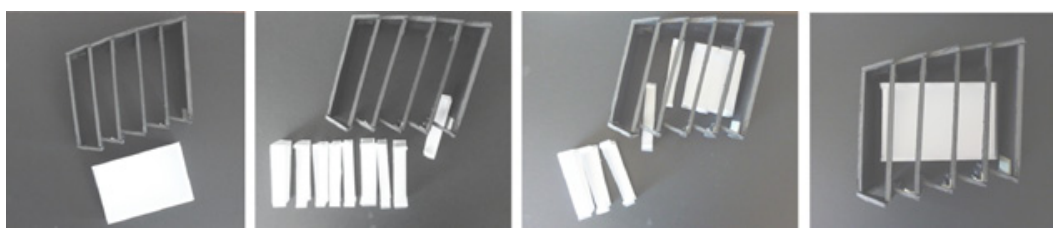

6.3.7 


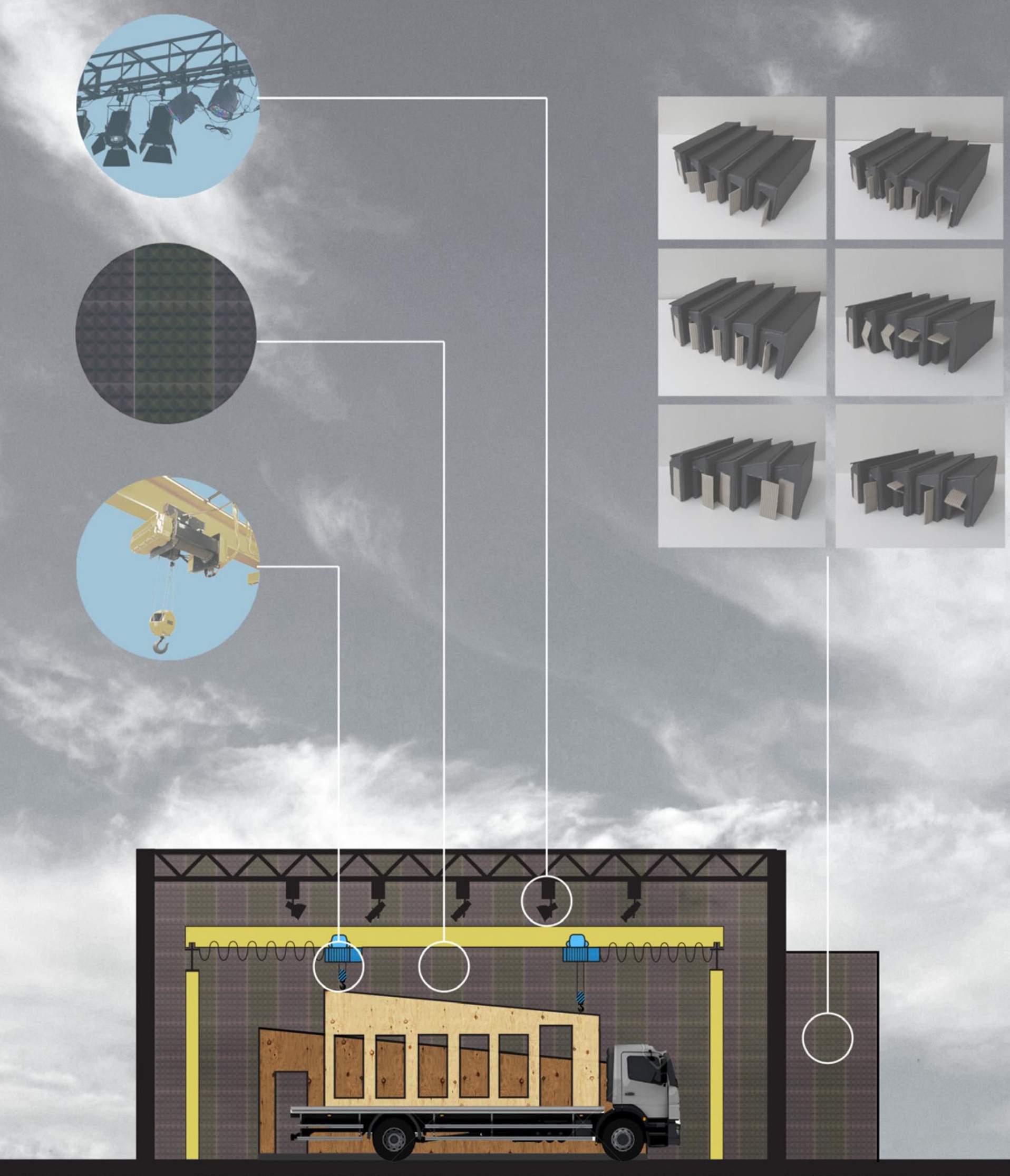




\section{Artificial Daily Ritual}

"The operation of deterring every real process via its operational double, a programmatic, metastable, perfectly descriptive machine that offers all the signs of the real." (Baudrilliard 2) (Figure 6.3.9)

Control of light intensity, colour and periods of wake and sleep allow researchers to manipulate the lighting environment to aid in movement and orientation within the sleep lab. The lighting canopy allows directional movement of light within interior, utilizing Knowles theory of migration in the home to guide residents and allow purposeful mobility within the sleep lab (Figure 6.3.10).

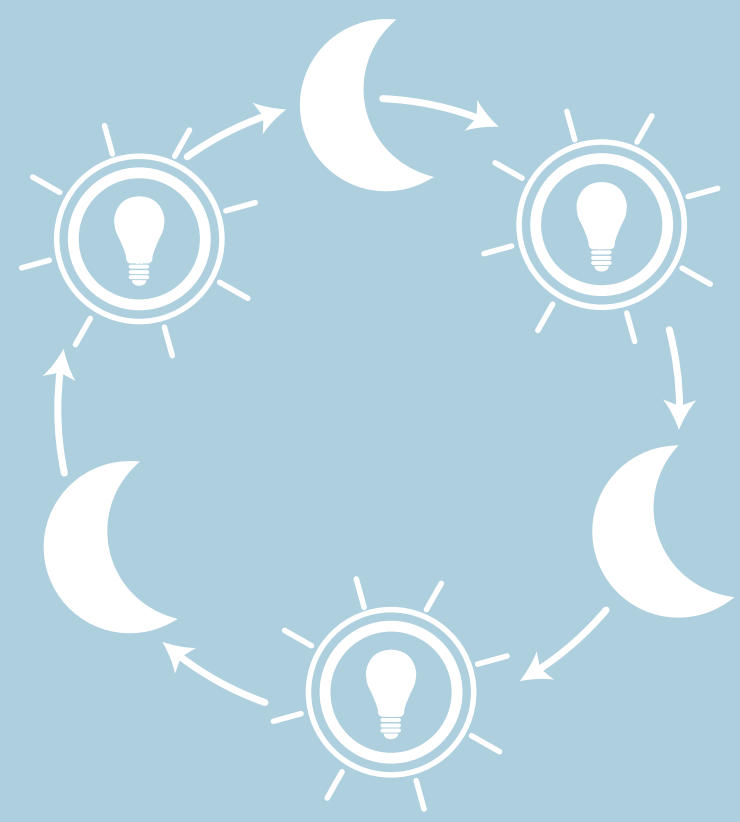

6.3.9 Artificial daily ritual diagram 

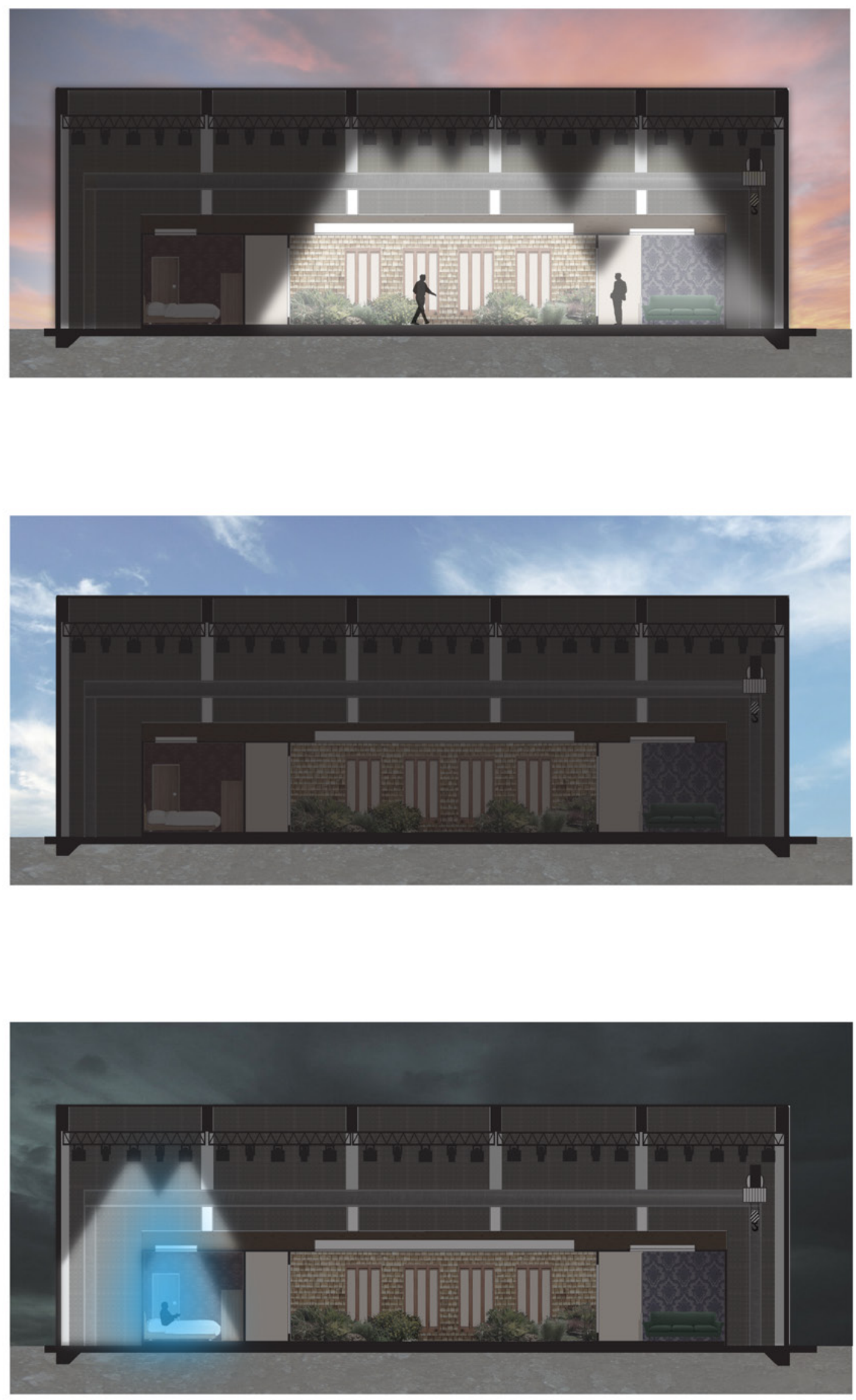

6.3.10 Section showing artificial daily ritual for resident 


\section{Artificial Domesticity}

Iterative massing studies of the external form tested methods to reduce the impact of the large $340 \mathrm{~m}^{2}$ area of the lighting lab within the respite facility (Figure 6.3.11). The scale of the structure coupled with the need to provide a spacious interior to accommodate future lighting lab experiments required simple geometric roof profiles that could accommodate these requirements. Other methods to reduce the mass of the building were to test variations in exterior cladding, openings that mimicked windows and doors, and reducing the number of garage door openings to establish a sense of domesticity to the exterior of the lab. At night however, this seemingly domestic exterior is transformed through light emitting panels to create a unique lighting spectacle for passers-by, drawing interest and attracting the community to this stimulating outdoor environment (Figure 6.3.12).

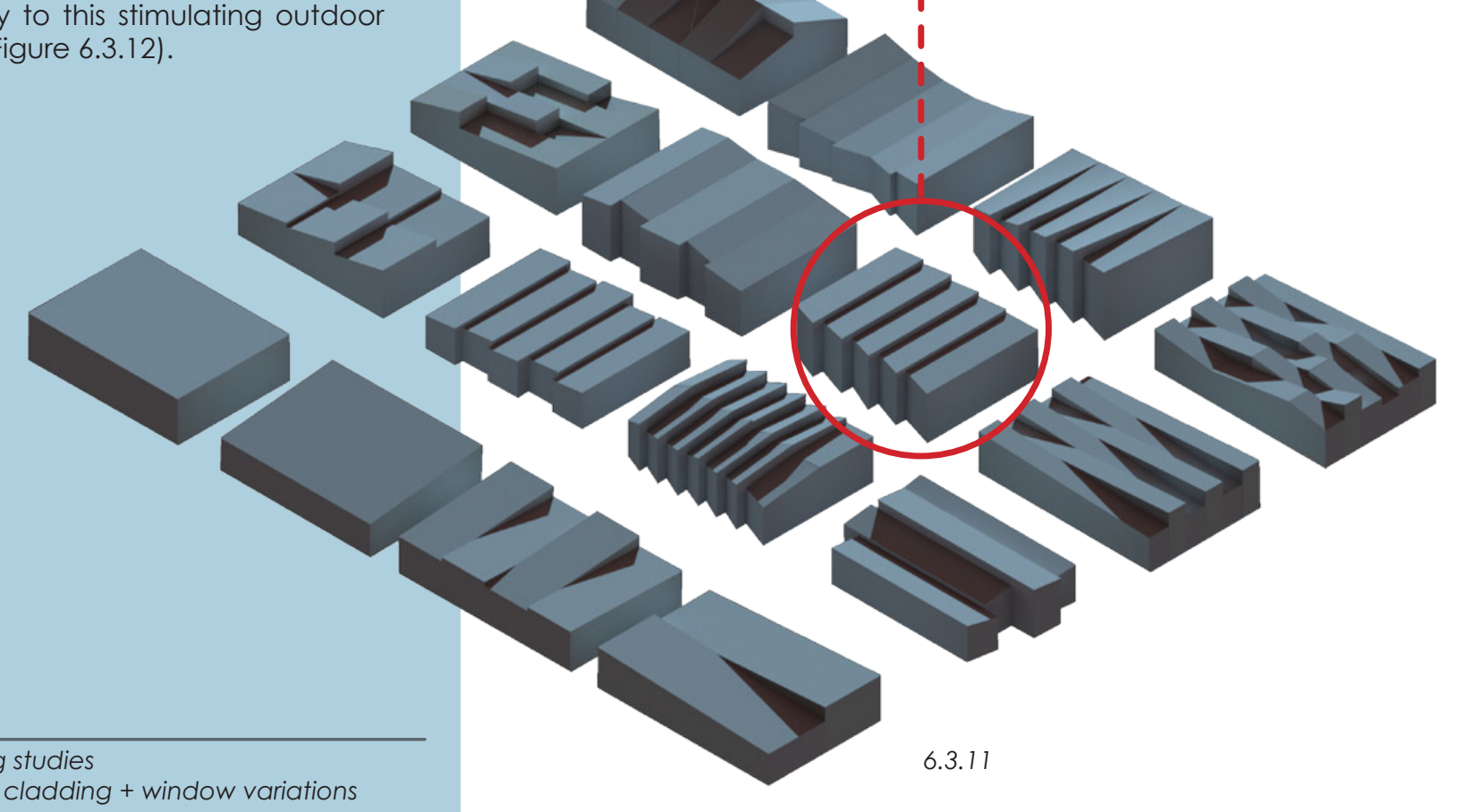




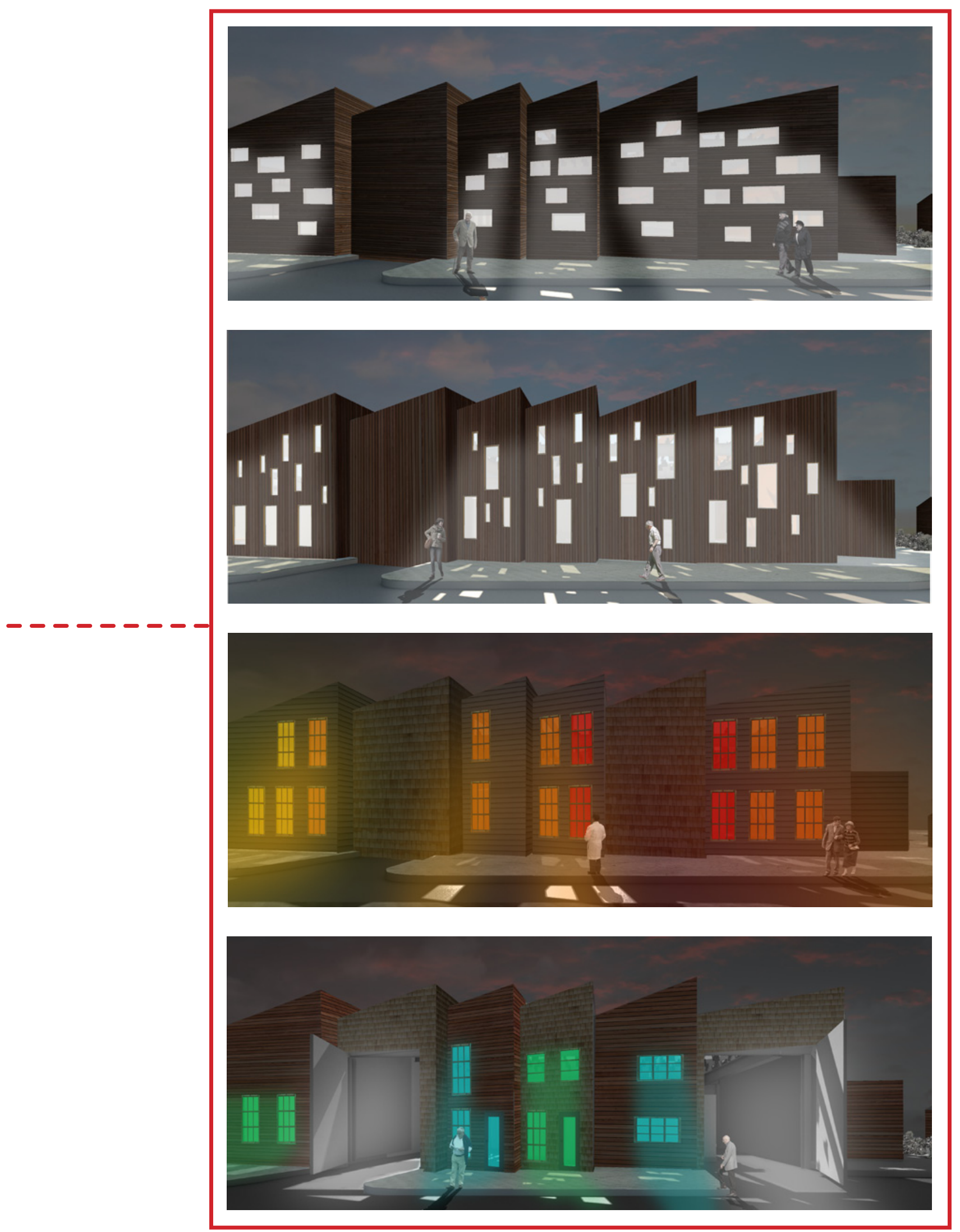

6.3 .12 


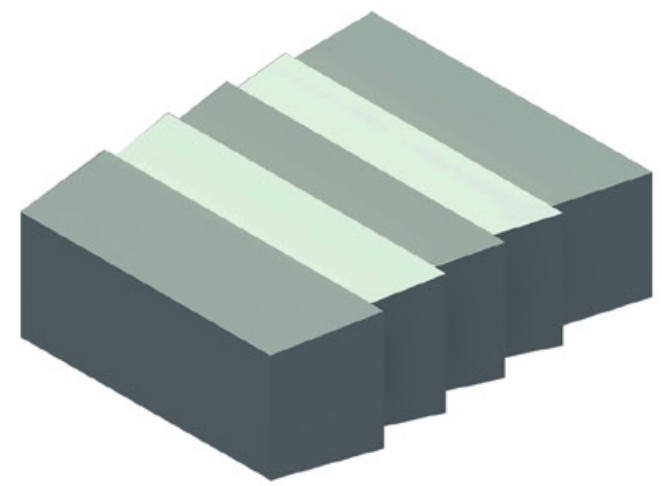

Exterior Form

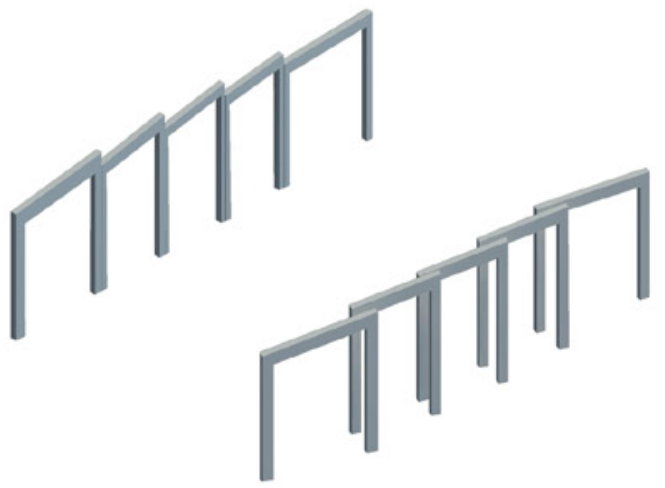

Moment frames $\mathrm{x}$ direction

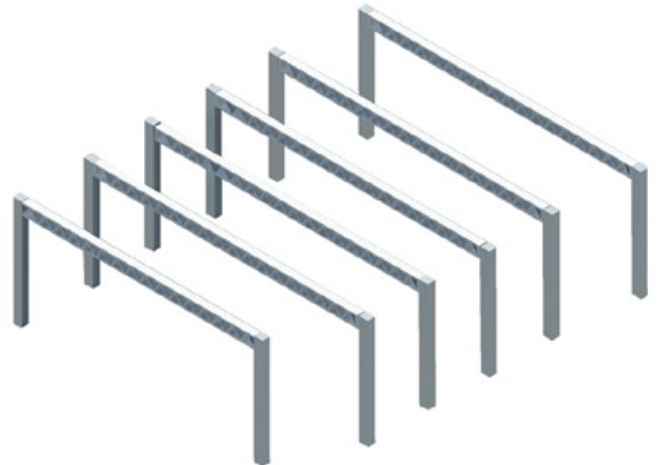

Moment frames y direction

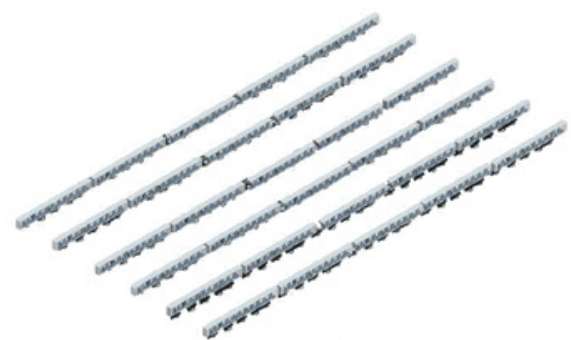

Roof mounted stage lighting

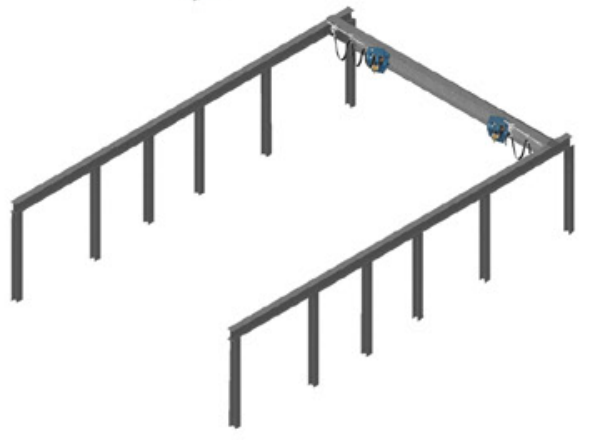

Overhanging crane

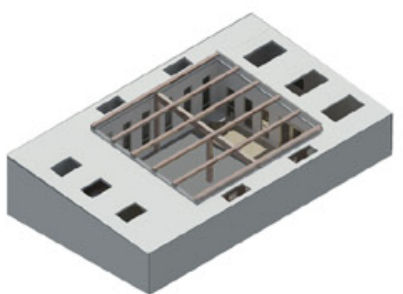

Sleep lab 


\section{Reflection + Summary}

The process of simulation offered a potential solution to developing sleep therapy research and treatment for moderate symptoms of dementia. Previous analysis of precedents such as De Hogeweyk and the Massey University Sleep /Wake Research Centre grounded this model of therapy alongside real world cases to suggest its appropriateness within dementia care. The design methods were successful in creating an environment that imitates natural settings to reduce the artificial aesthetic of the sleep lab, therefore improving the residents wellbeing within $a$ seemingly natural environment. The process also improved the desired flexibility necessary to provide a person centered approach towards the sleep lab, allowing experimentation to cater to generational groups and individuals through material, form and layout alterations.

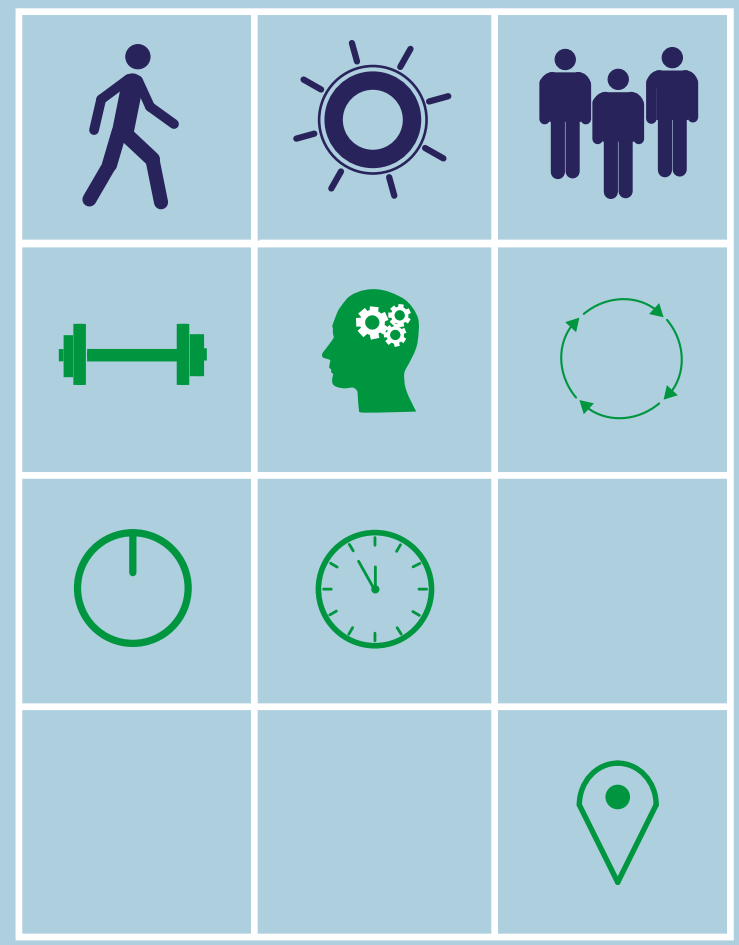




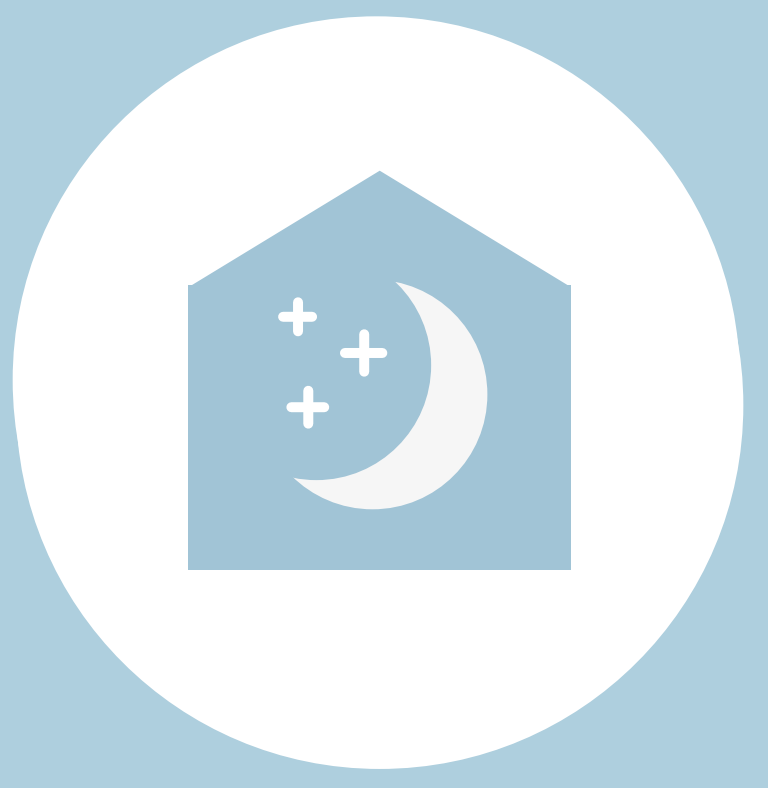




\section{7}

\section{Guiding Light}

Guiding Light embodies the accumulation of previous tests and experiments into the design of New Zealand's first sleep therapy and research centre for dementia. The developed design presents an alternative model for respite care that emphasizes light, movement and community as environmental strategies to improve sleeping behaviour and quality of life for those affected by dementia. 


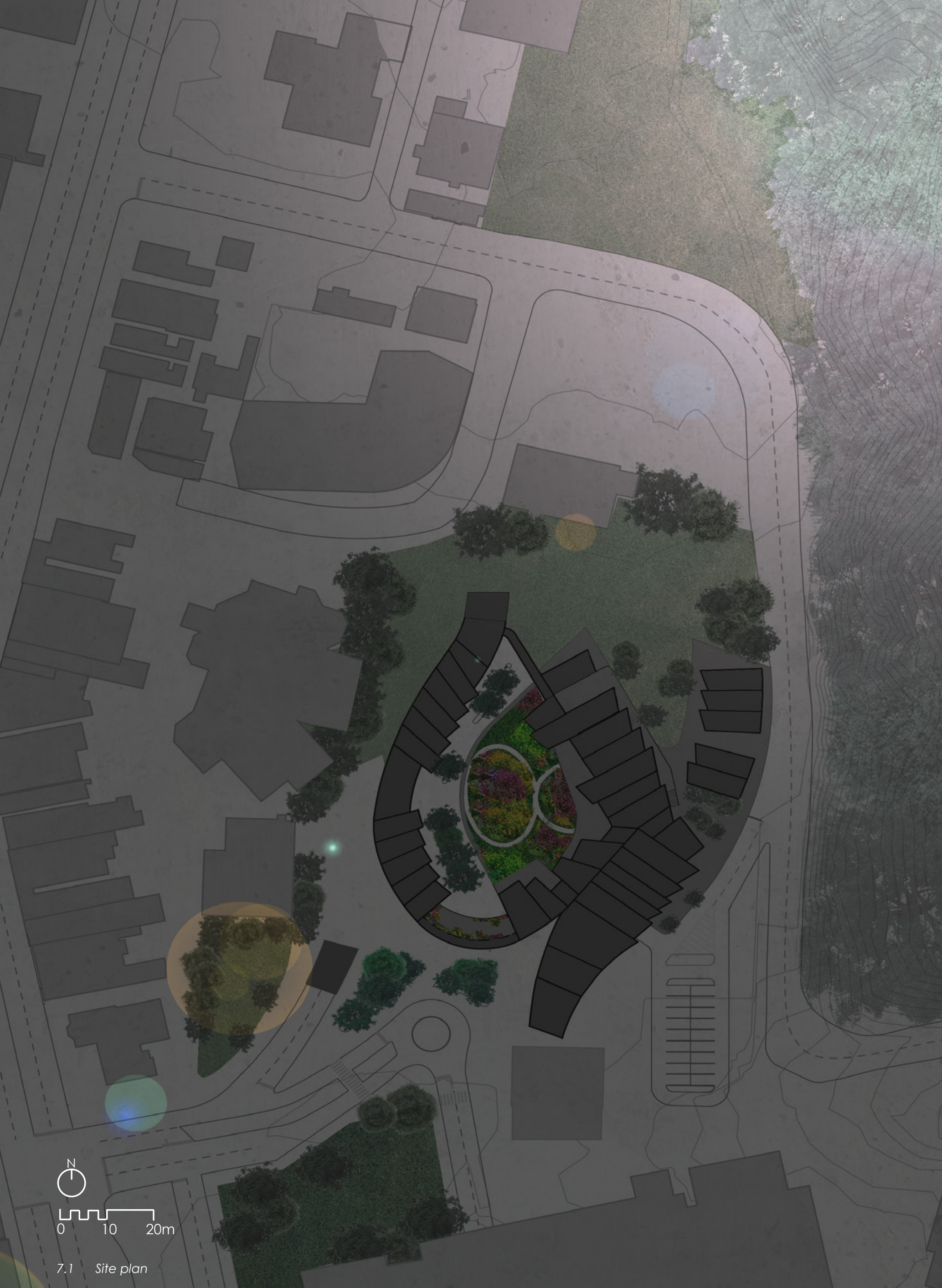




\section{5:}

As morning light slowly dawns, sunlight breaks through the northeast ridgeline to reveal a building with curves, open courtyards and an abundance of vegetation that blurs the boundary between the town belt and urban environment, the building nestling amongst its natural surroundings (Figure 7.1 $-7.2)$. 


\section{z}

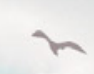

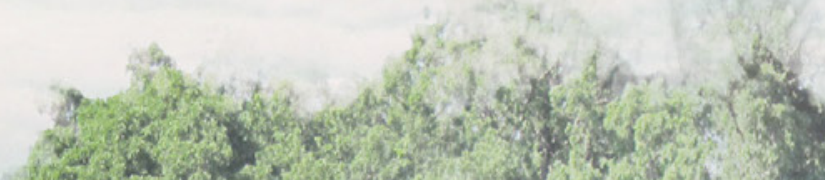

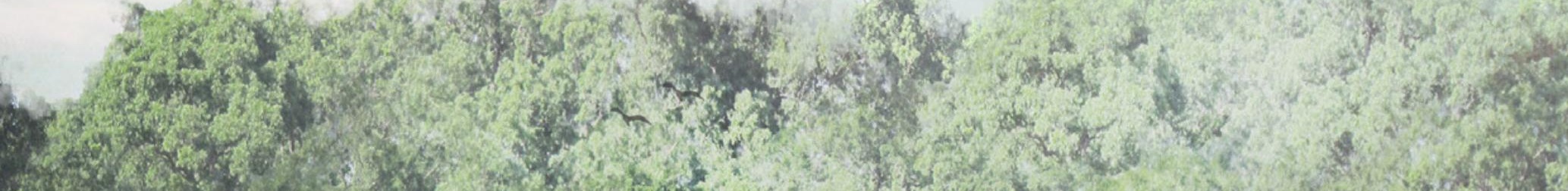
3.5.

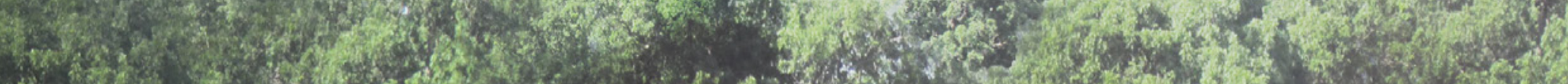

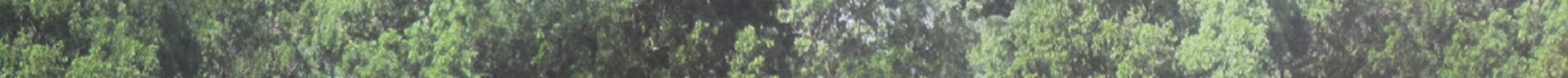
(2)

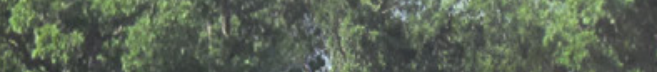
3.

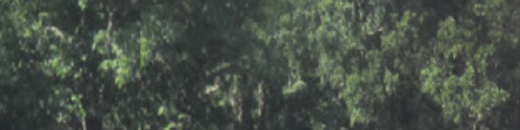
tos

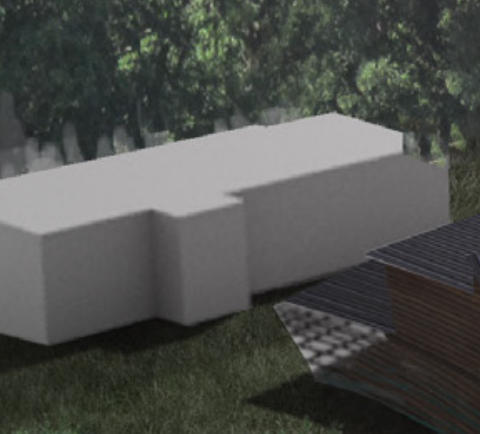

6. 46. T. Th the of 2. (15. (3) 5

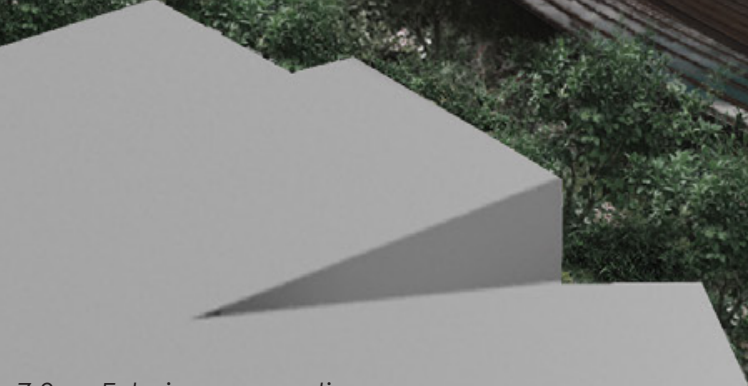

7.2 Exterior perspective Her. S6
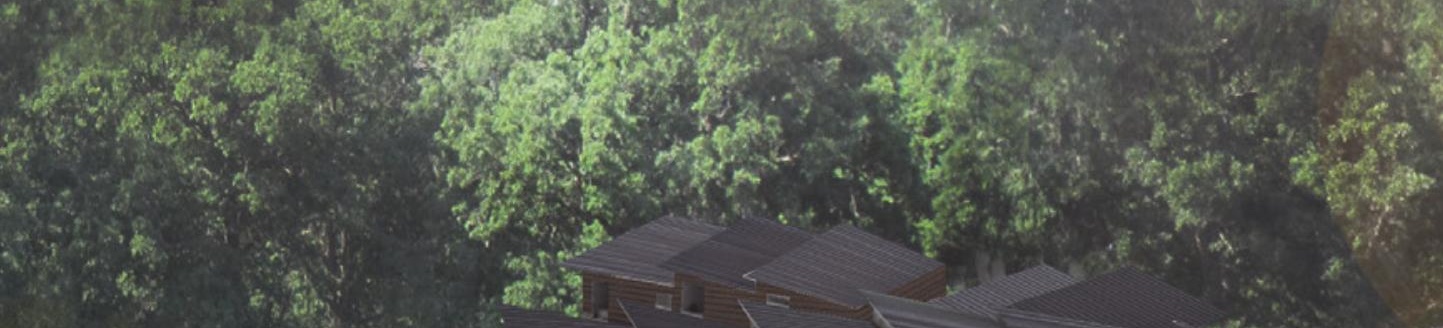


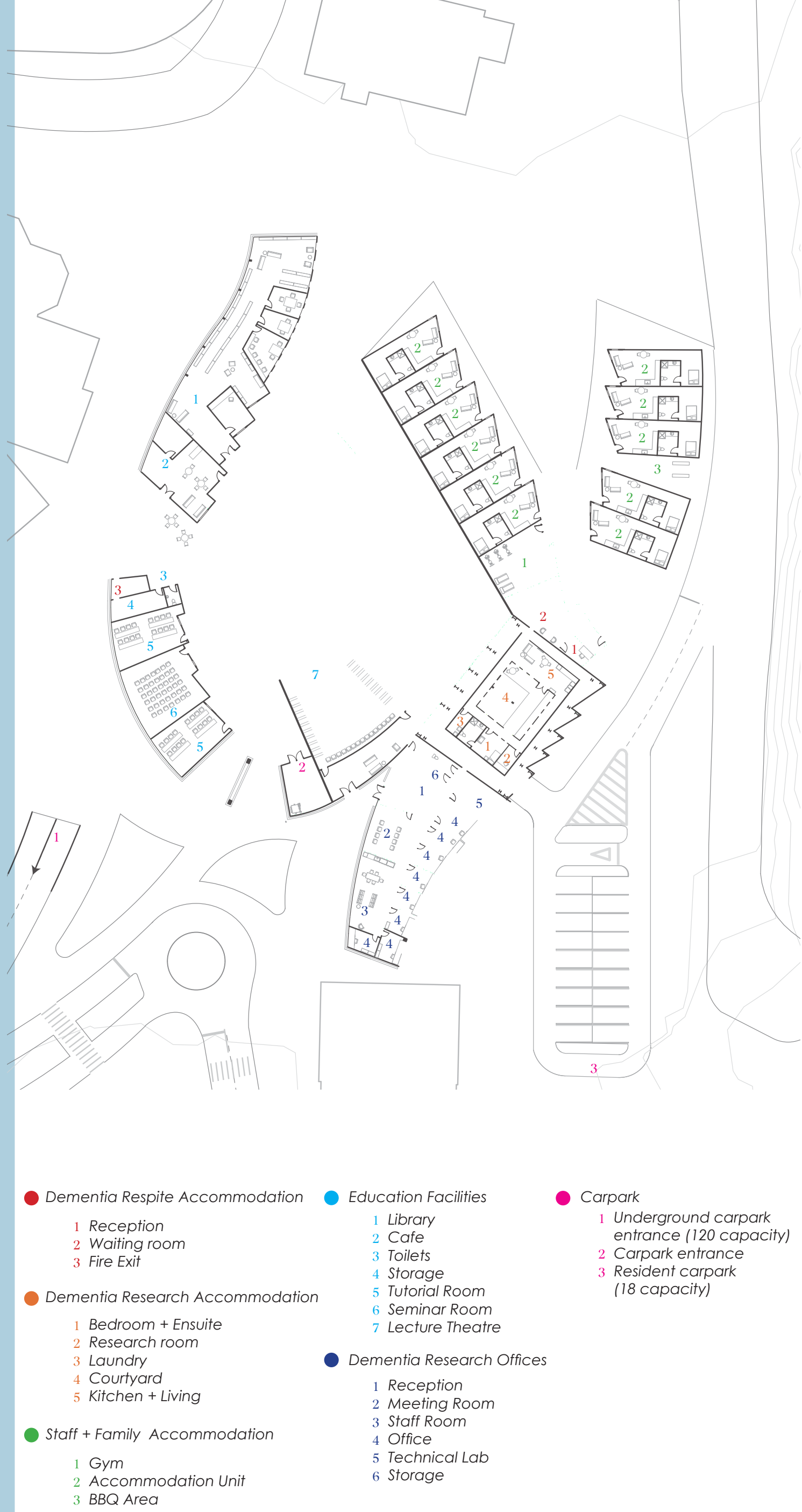




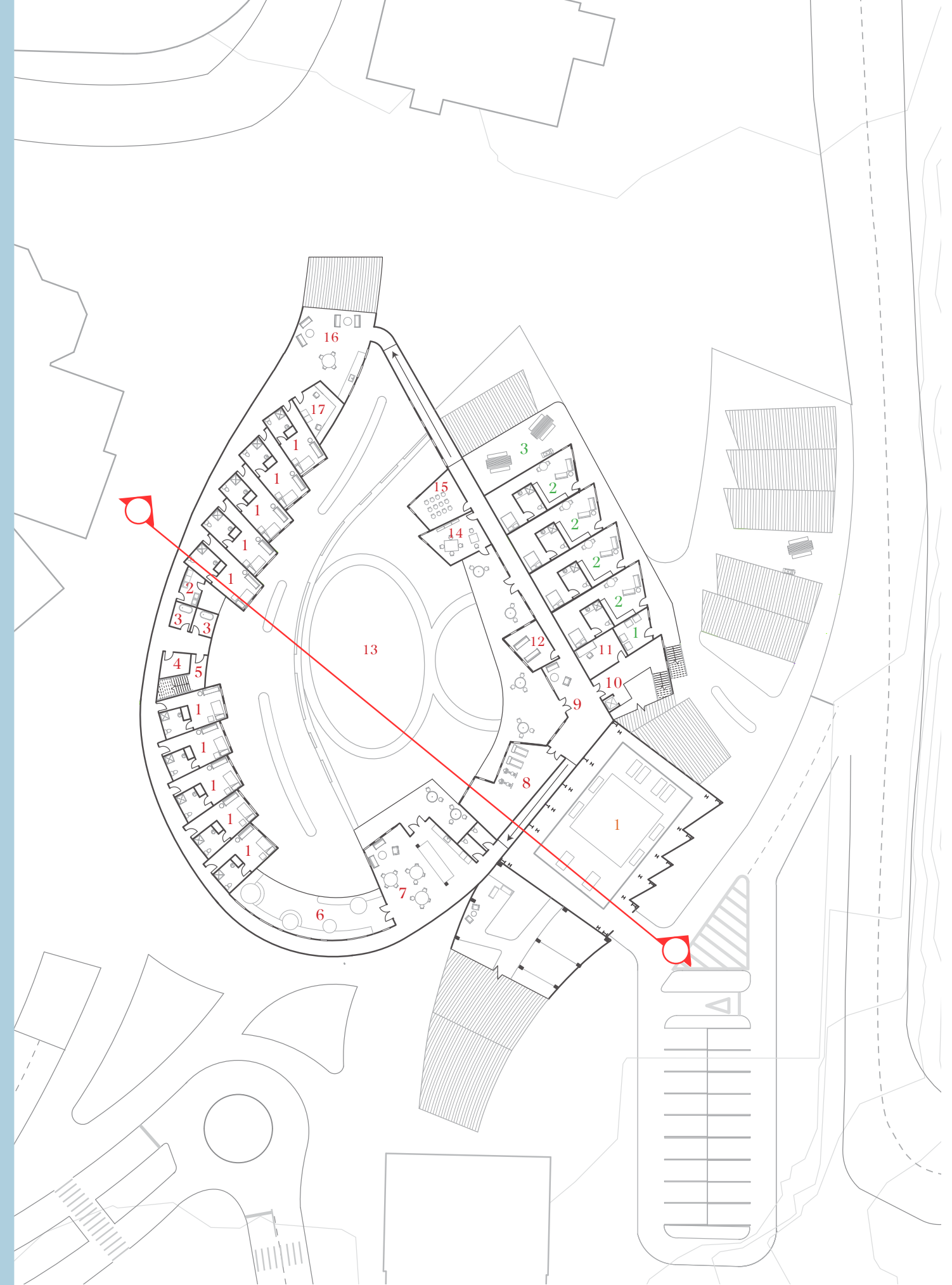

Dementia Respite Accommodation

$\begin{array}{ll}1 & \text { Bedroom + Ensuite } \\ 2 & \text { Laundry } \\ 3 & \text { Bathroom } \\ 4 & \text { Storage } \\ 5 & \text { Fire Exit } \\ 6 & \text { Vegetable Garden } \\ 7 & \text { Kitchen/Living } \\ 8 & \text { Gym } \\ 9 & \text { Living Room } \\ 10 & \text { Entrance } \\ 11 & \text { Staff Room } \\ 12 & \text { Counselling Room } \\ 13 & \text { Shared Courtyard } \\ 14 & \text { Craft Room }\end{array}$

Bedroom + Ensuite

Storage

Fire Exit

Vegetable Garden

7 Kitchen/Living

8 Gym

9 Living Room

10 Entrance

12 Counselling Room

14 Craft Room

\begin{abstract}
15 Entertainment Room
16 Morning Room

17 Staff Room
\end{abstract}

Dementia Research Accommodation

1 Sleep Lab

Staff + Family Accommodation

1 Laundry

2 Accommodation Unit

3 BBQ Area 


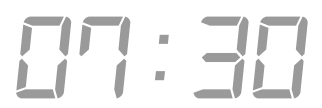

Morning light filters through windows that are positioned to maximize natural light exposure for residents sleeping in bed (Figure 7.5 - 7.6). The positioning of windows on both east and north facing walls allow for seasonal variation of light, increasing the efficiency of natural light exposure during the year. As residents wake, they are greeted by familial surroundings of domestic building forms and garden space outside (Figure 7.7).

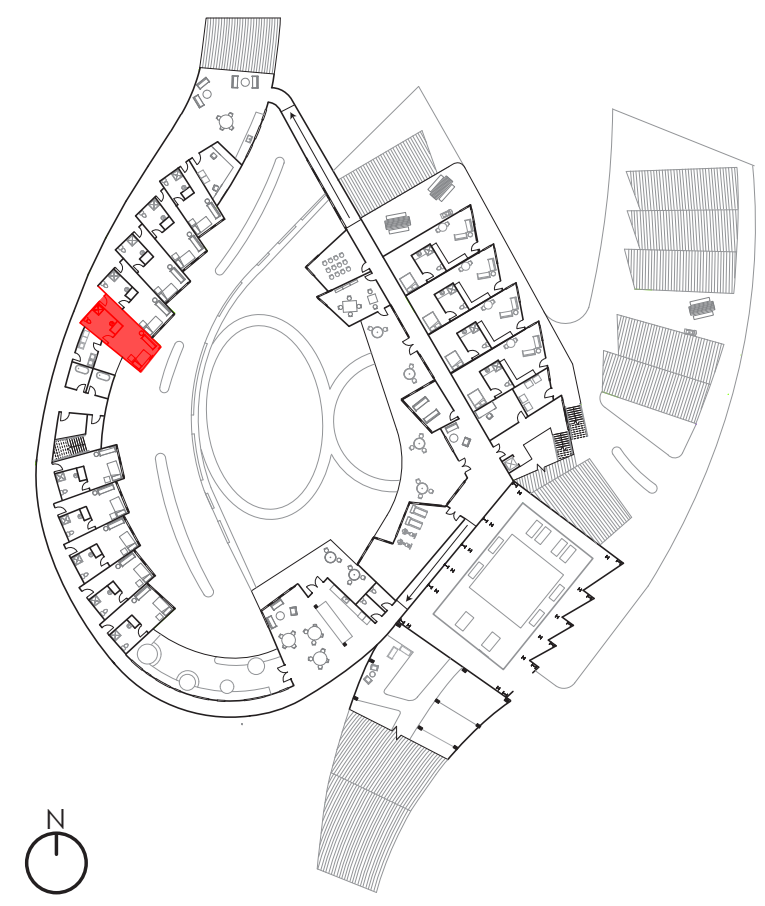

7.5 Bedroom orientation 


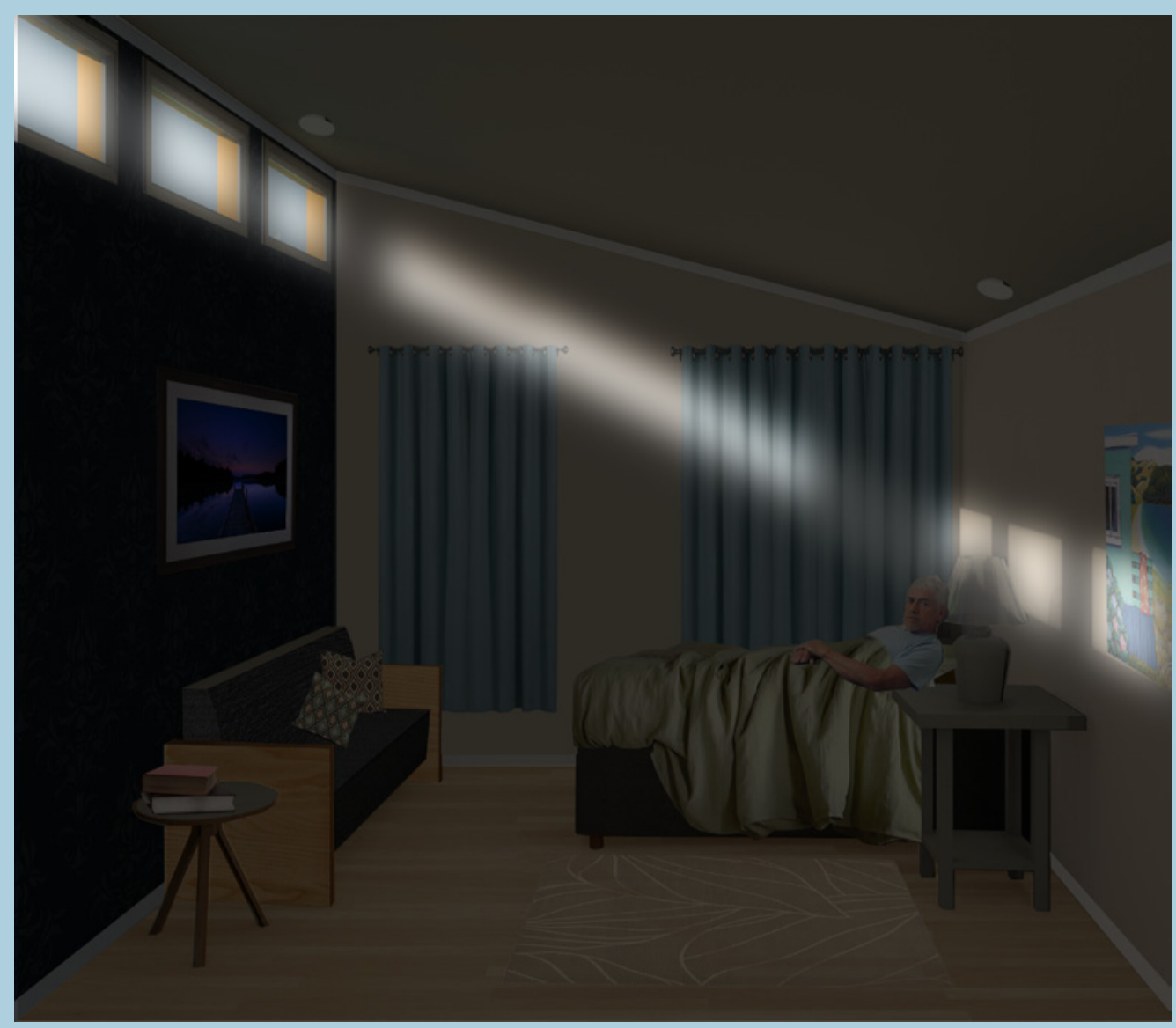

7.6 Morning light stimulation

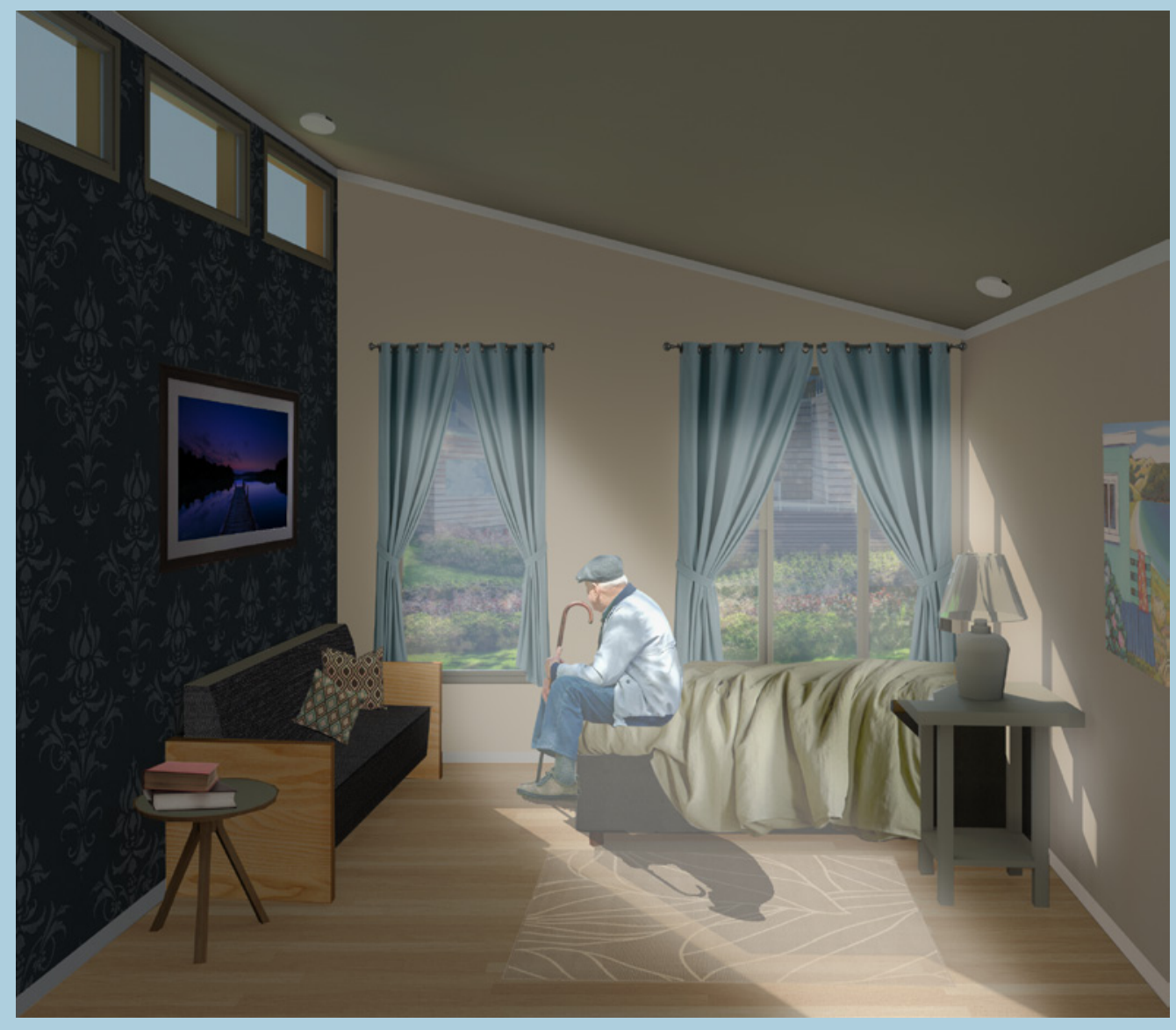

7.7 View towards courtyard 

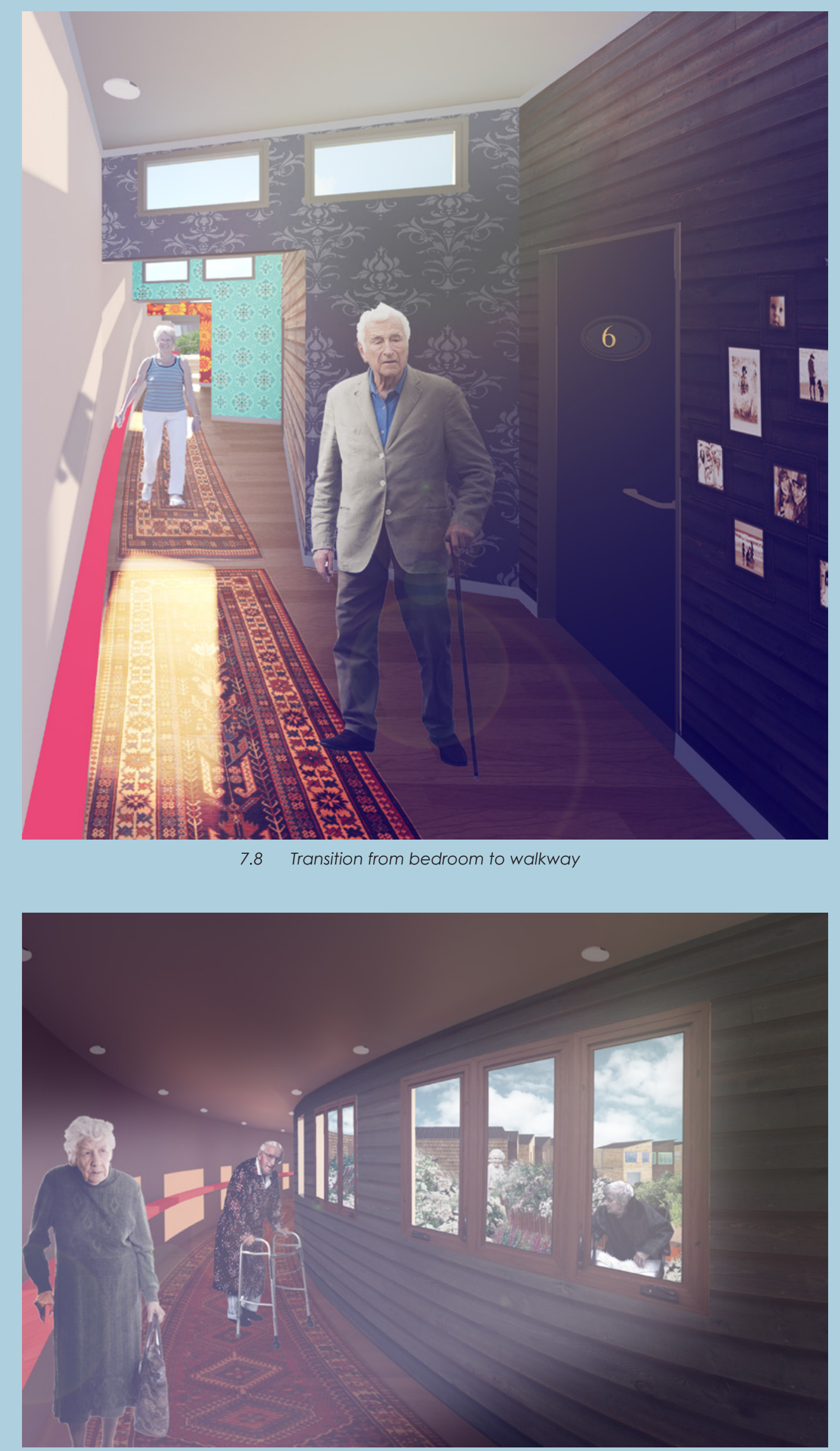

7.9 External view from walkway 


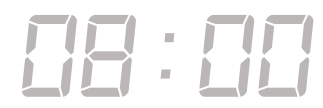

Upon leaving the bedroom, residents enter a walkway space that provides a continuous enclosed loop for wandering within the building (Figure 7.8). The walkway encourages increased mobility for residents as spaces are purposely dispersed to provide opportunities for exercise as residents move between spaces (Figure 7.10). The use of colour, natural light and environmental cues help stimulate residents, generating an implicit cognitive response that improves their orientation as they wander through the building (Figure 7.9).

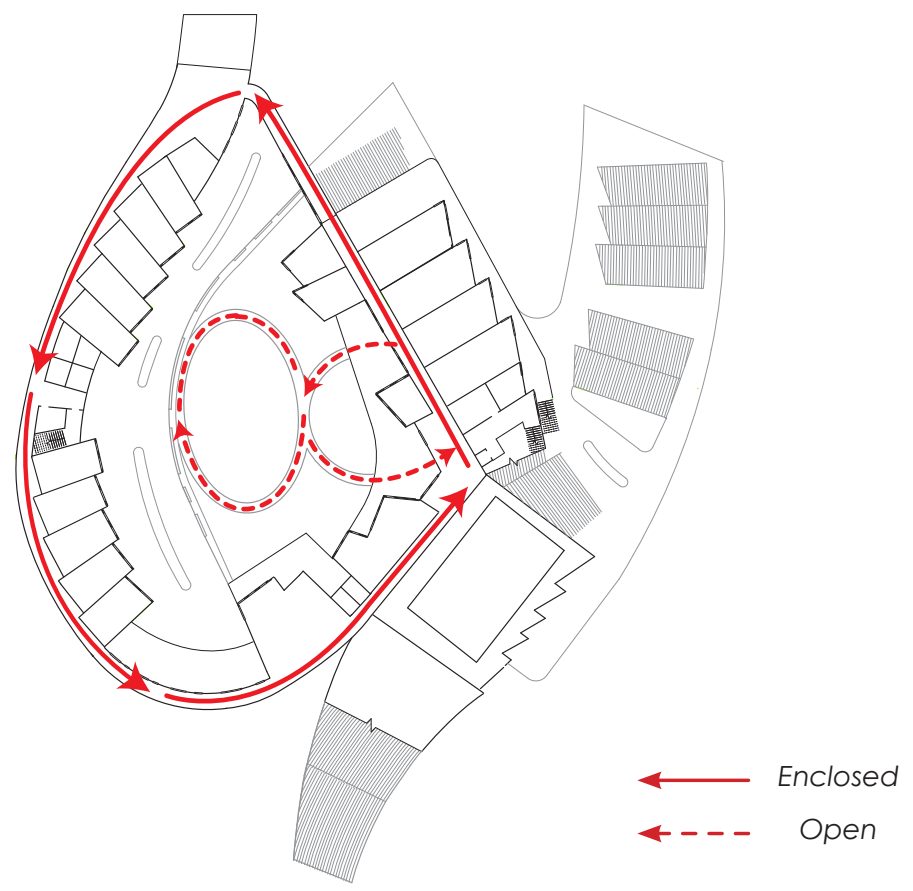

7.10 Walkway layout 


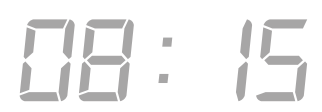

Natural light exposure is maintained through the course of the day as spaces are positioned and orientated to respond to the circadian rhythms of the sun (Figure 7.11). This follows Knowles's notion of the architectural interstitium as the building emphasizes the migration of residents to respond to changes in daylight. Areas of high use such as the open plan kitchen / living area are orientated in a northeasterly direction to maximize daylight exposure during morning and midday meal times (Figure 7.12). As the sun moves, spaces for daytime activities such as the gym are orientated in a northwesterly direction to maintain light exposure (Figure 7.13). In contrast, spaces for entertainment in the afternoon are orientated south to reduce light exposure in order to relieve sun-downing symptoms.

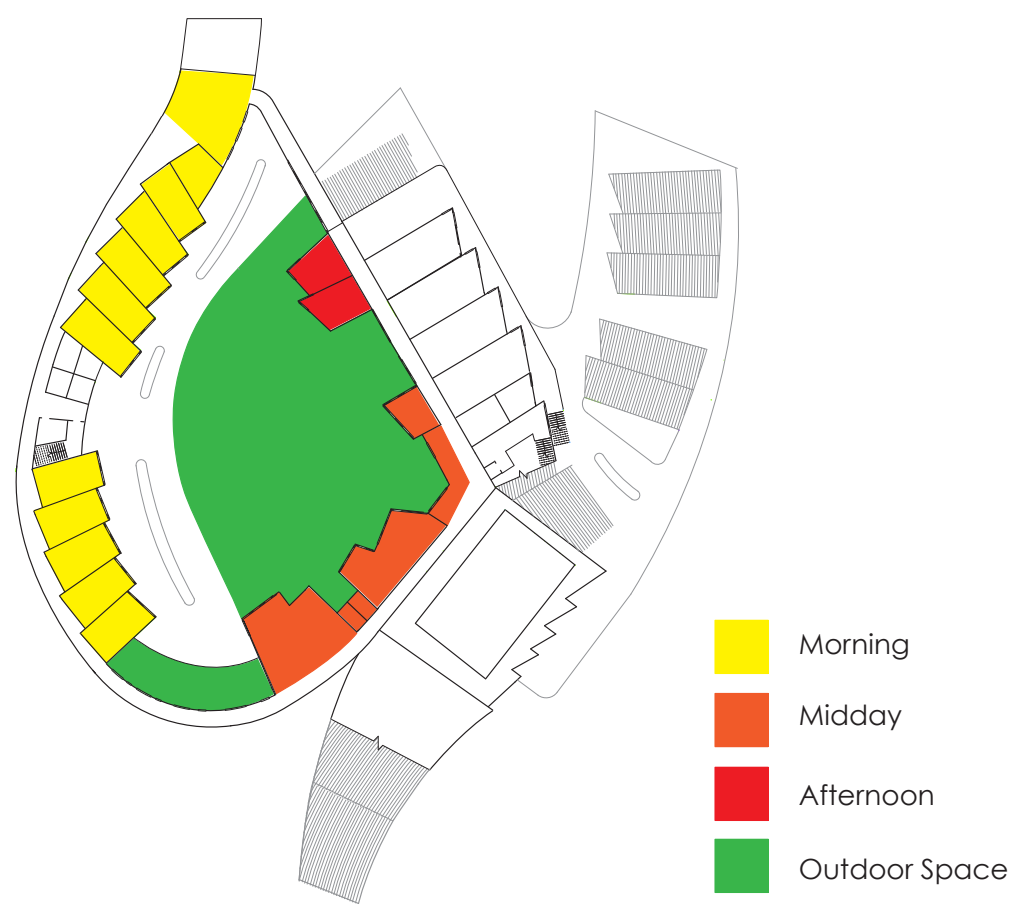

7.11 Natural light programmatic arrangement 

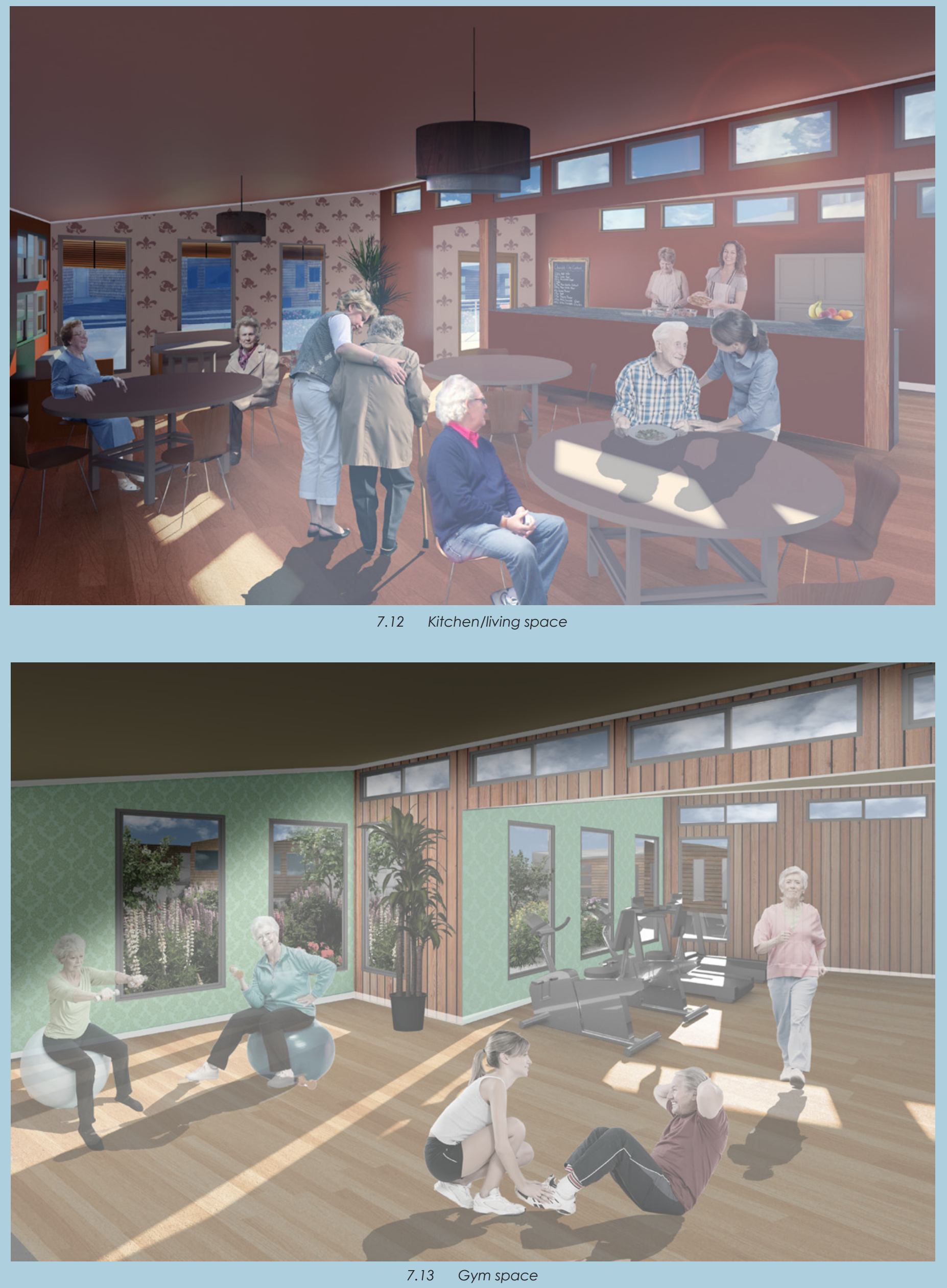


\section{T: ГT \\ II:LIL}

Views and access to a central courtyard space offer a multisensory environment for outdoor activities such as gardening, walking and meeting people from the medical community (Figure 7.14 - 7.15). Variations in building form, roof profiles and timber cladding aim to breakdown the institutional mass, creating a domestic scale and visual aesthetic for residents who occupying the inner courtyard.

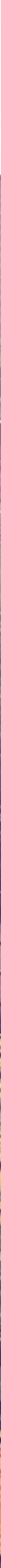




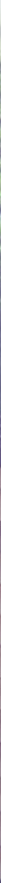

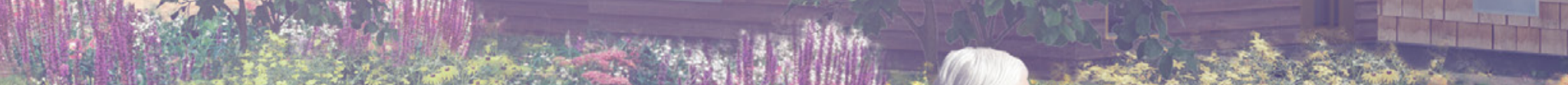

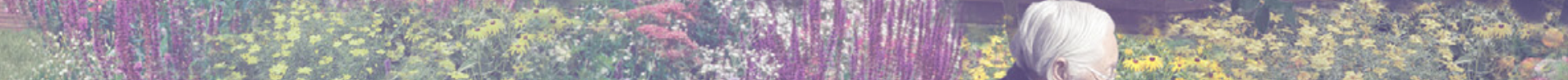
1.

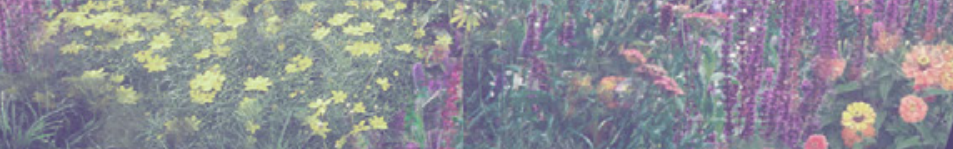
(1) 1 (2)

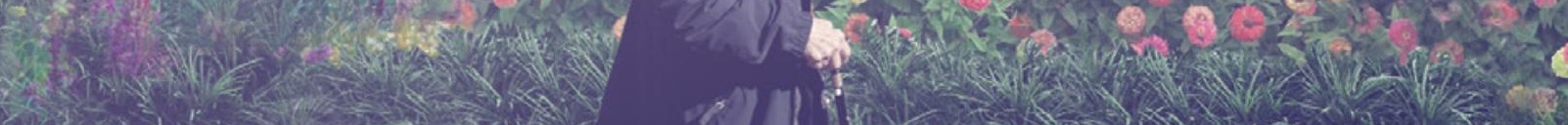

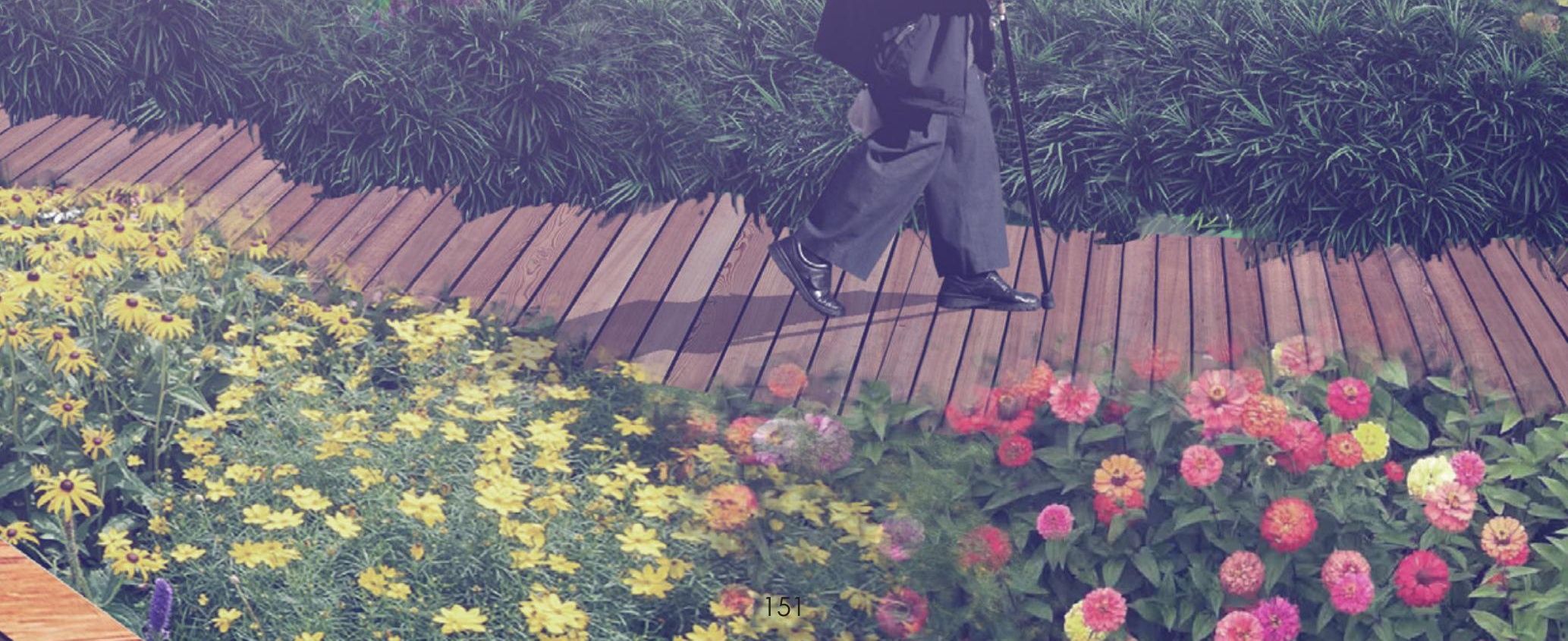




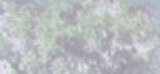

(x)

(1)

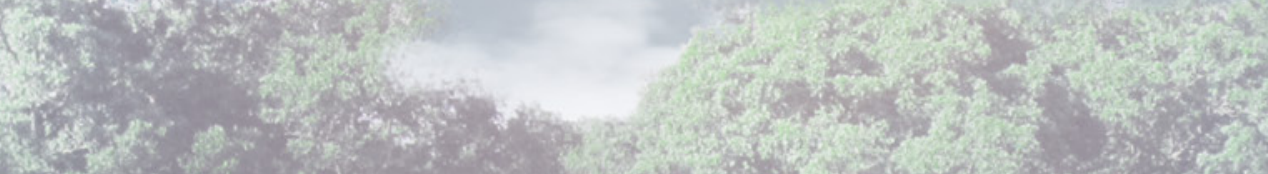

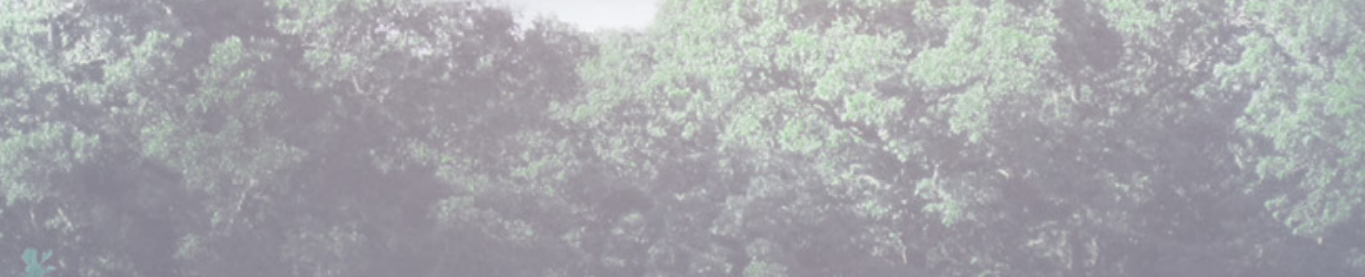

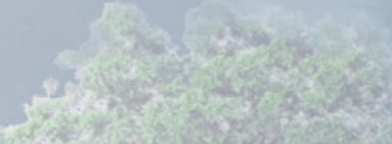

(x)

intasing

25.

$x^{2} e^{2}=$

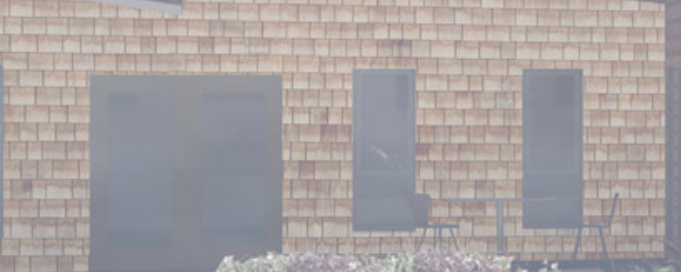

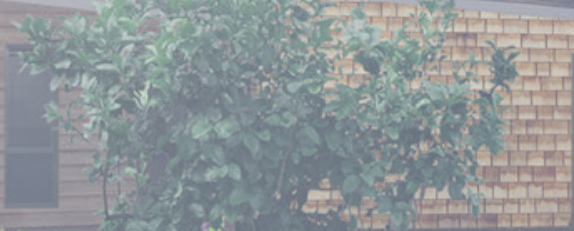

Evin

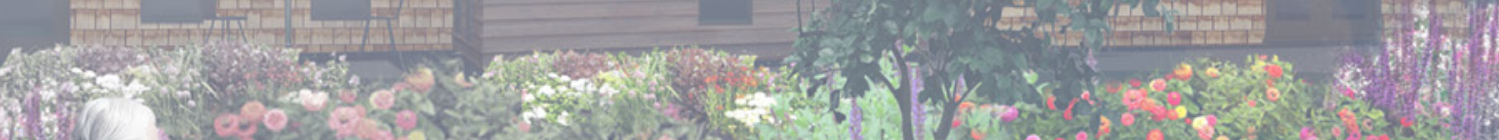

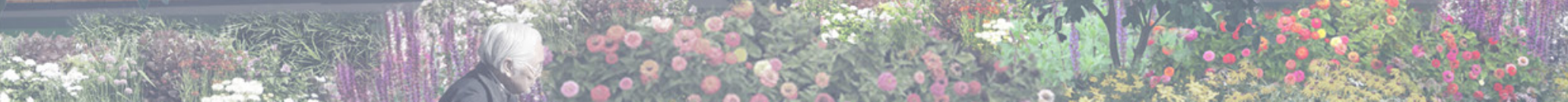

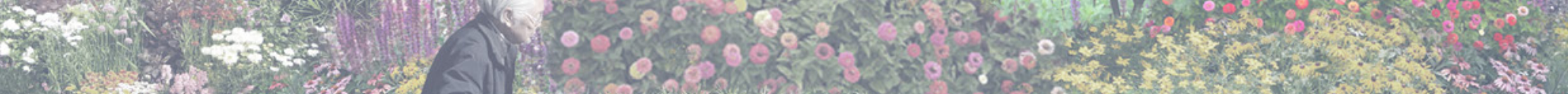

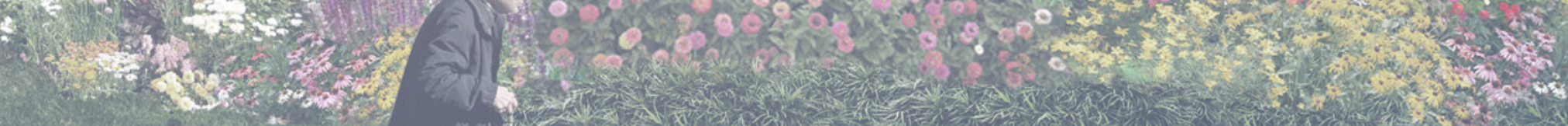
4.

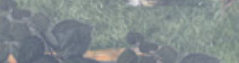
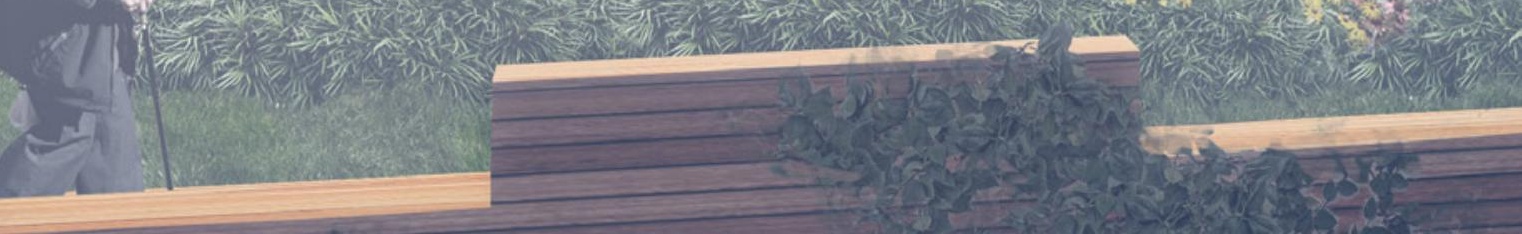

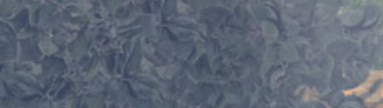

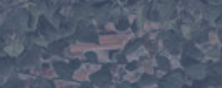

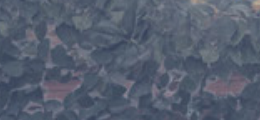

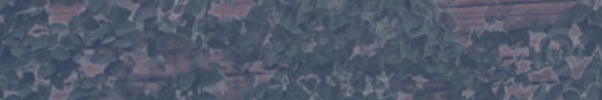

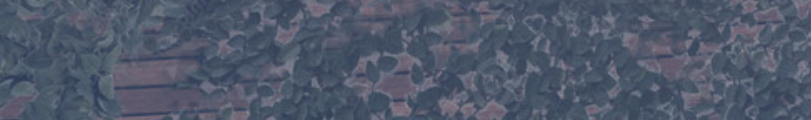

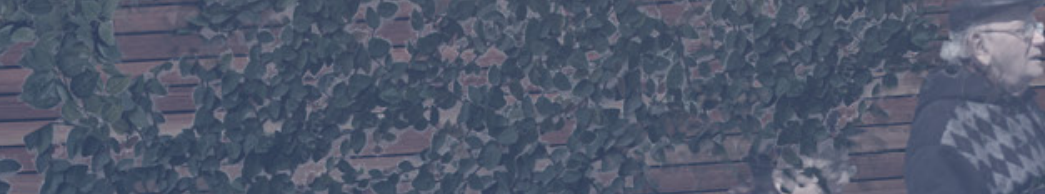

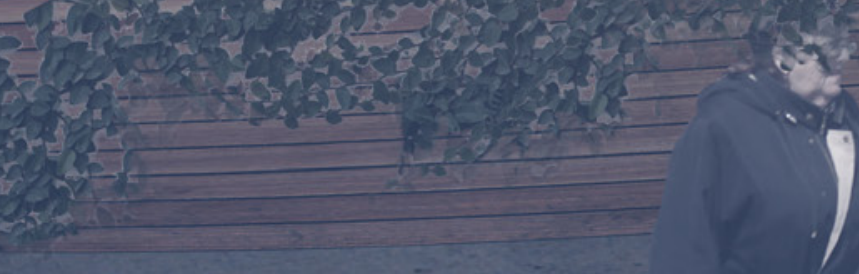

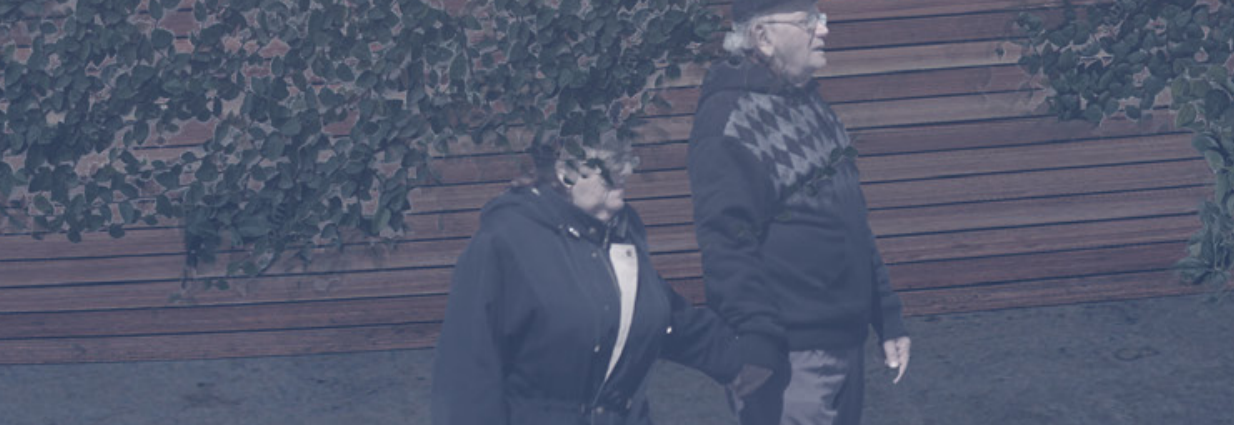

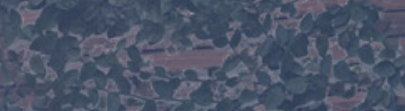




\section{$14: 50$}

In contrast with the inner domestic courtyard space, the institutional scale and aesthetic of the exterior provides a unique building image within the medical precinct. The exterior profile with its curved geometric form and timber cladding draws interest and movement around the building as the repetitive rising roof profiles lead the eye to the sky above (Figure 7.16).

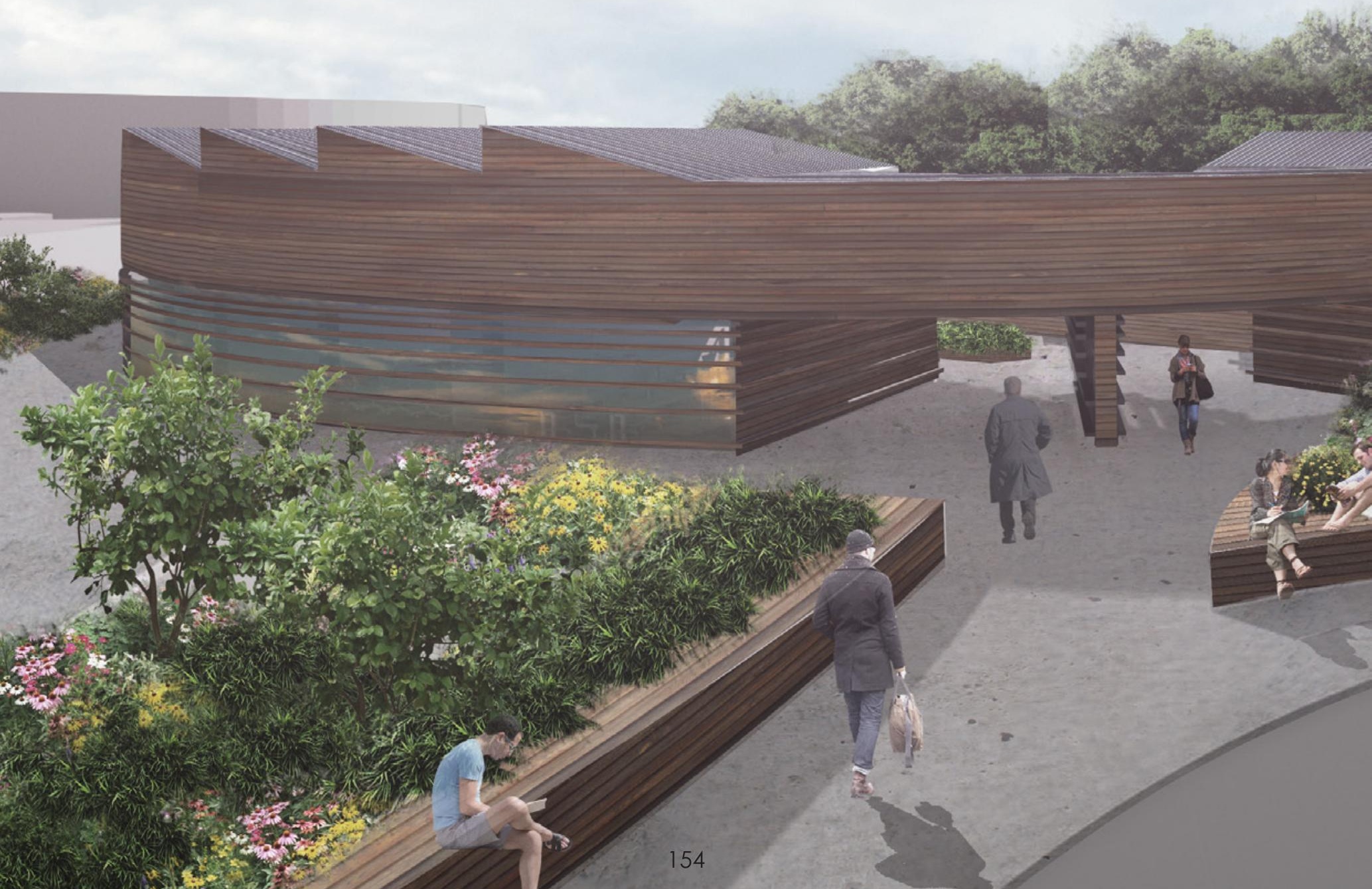




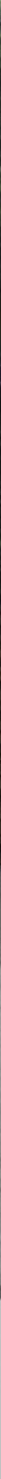




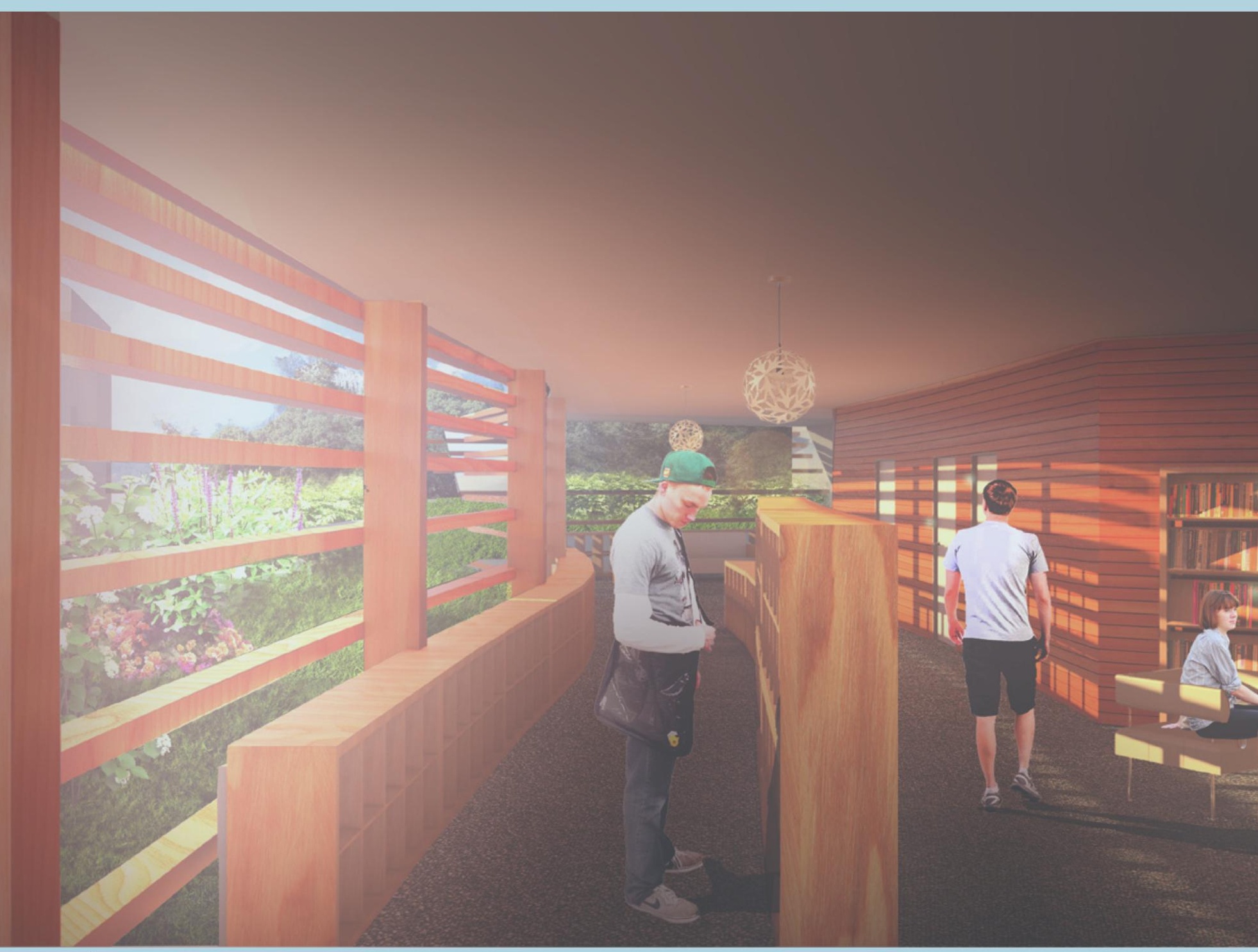

7.17 Library space 


\section{结: 识}

The integration of educational programs helps to promote a positive image of the dementia facility within the medical precinct, with dedicated teaching spaces, café and library providing a hub for learning and social interaction within the community. The interaction of the community with residents is achieved through the shared courtyard, with education facilities located on the ground floor for improved accessibility and security for dementia residents in respite. Visual exposure to dementia residents and the domestic courtyard are balanced by connections to the external environment, with large glazed panels providing natural light for a comfortable learning environment (Figure 7.17). 


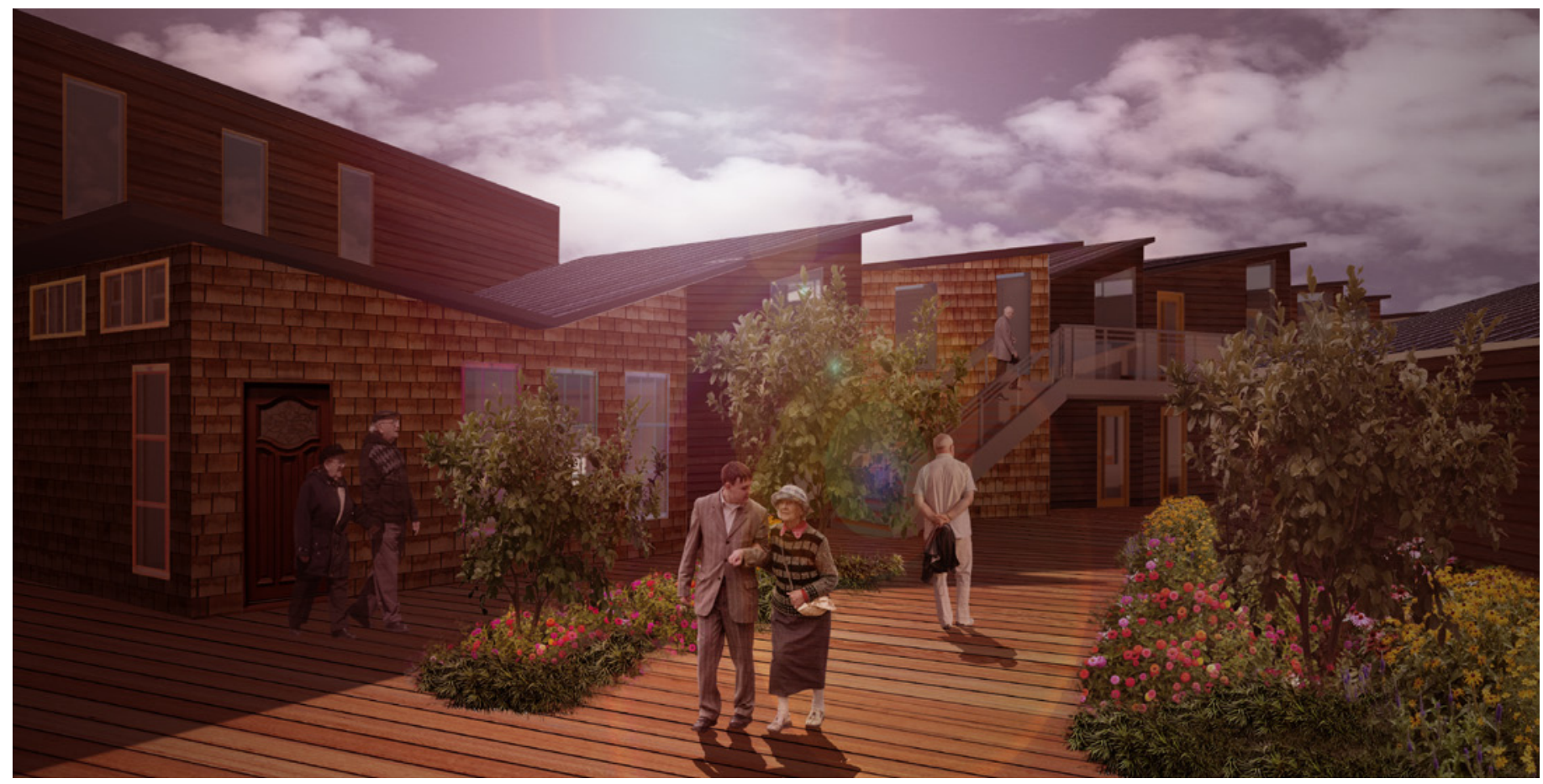

7.18 Entrance exterior perspective

\section{怗: 滥}

The approach for dementia residents is situated within a domestic scale, as variations in timber cladding, roof profiles and open walkways breakdown the building form to provide a domestic aesthetic upon approach (Figure 7.18 7.19). The design seeks to reduce the anxiety and stress caused when people with dementia are separated from their family as a shared entrance with other families, staff and medical professionals provides a sense of community upon approach.

Individual flats form an open courtyard space with green areas for relaxation and physical activities, creating opportunities for social exchange and a sense of community for families, students, staff and medical professionals that occupy the flats (Figure 7.20). Open planned living and kitchen spaces face the open courtyard to increase the notion of a social hub between flats, with bedrooms located away from courtyard for added privacy (Figure 7.21).

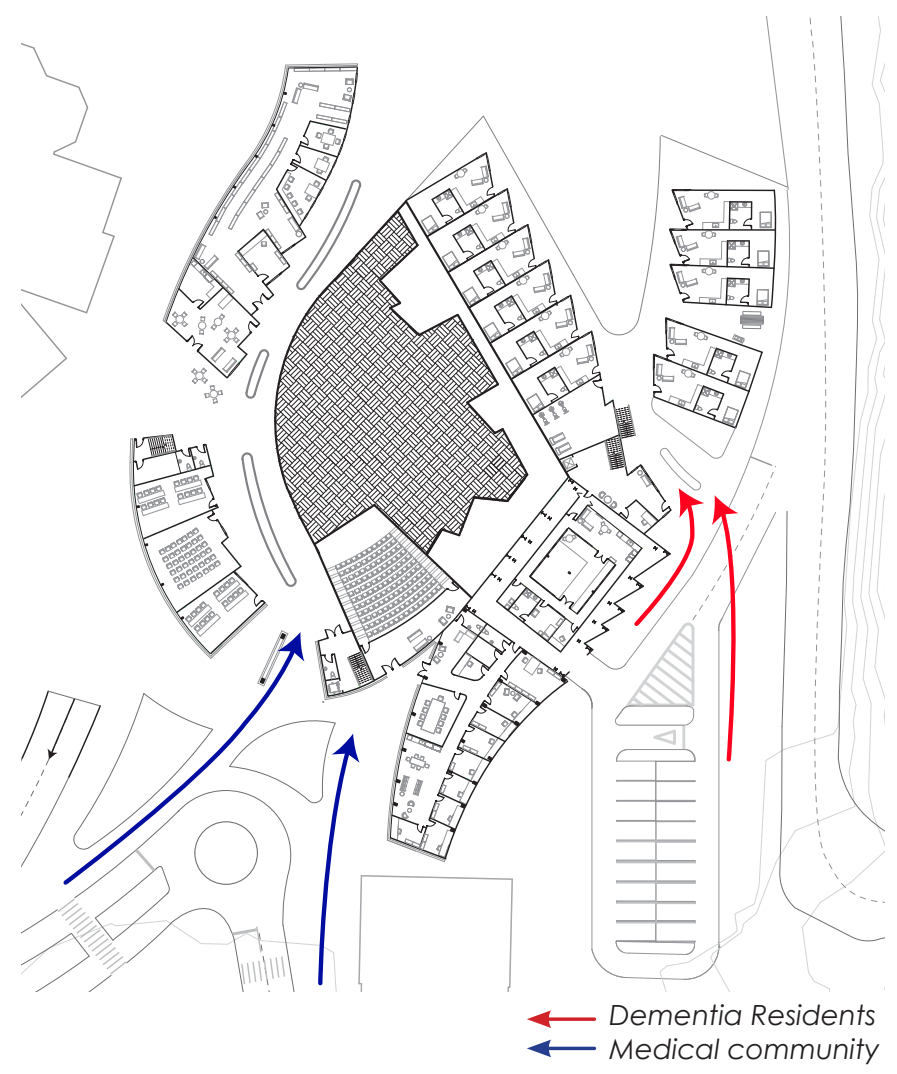

7.19 Separate entrances 

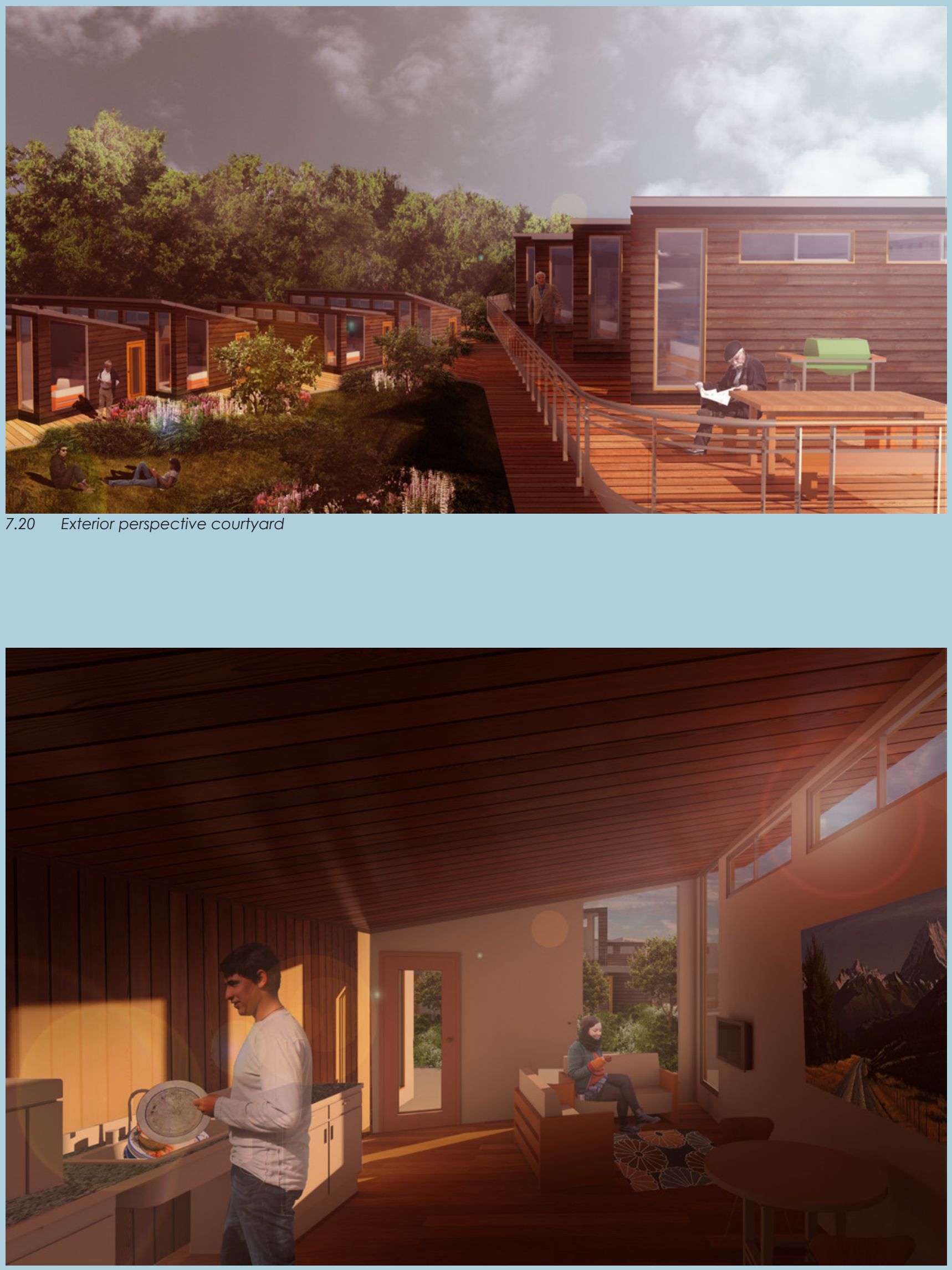


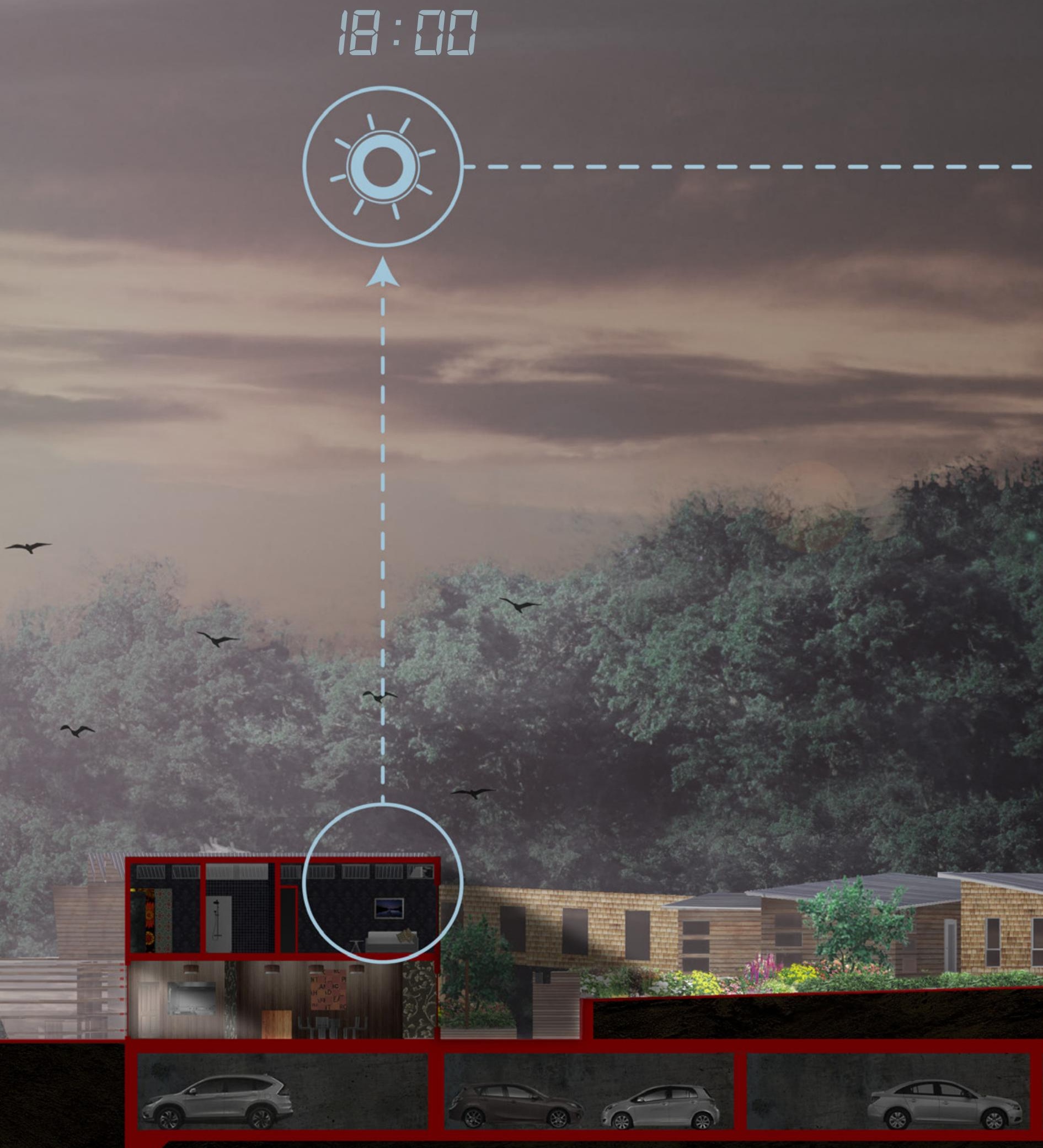

பேே

7.22 Cross-section 

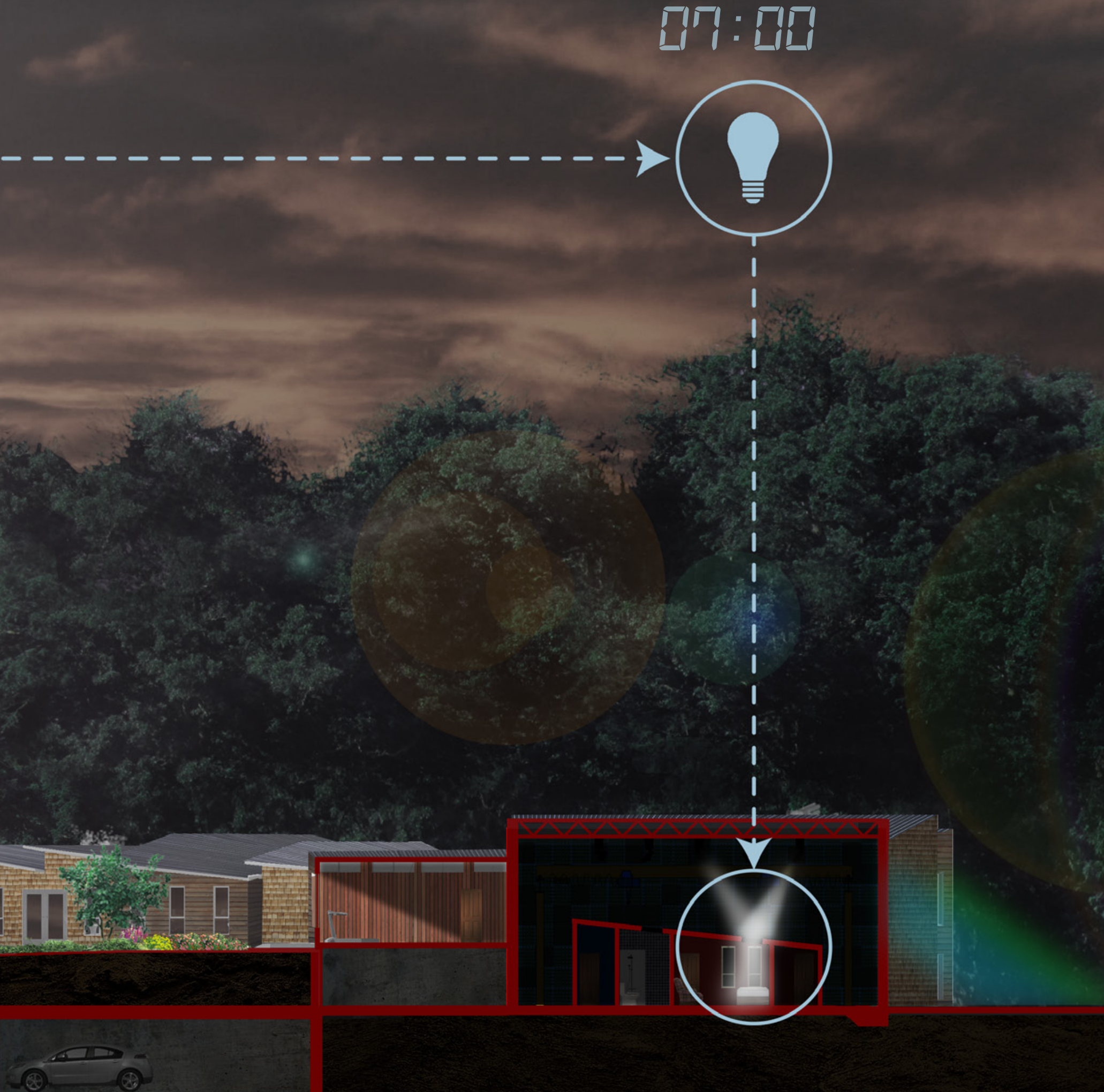

As dementia progresses, the circadian rhythm of a resident becomes increasingly detached from the circadian rhythm of the sun. To adapt to this inevitable deterioration, residents with more moderate symptoms are transitioned into the sleep lab, an artificially lit environment that simulates their unique circadian rhythm (Figure 7.22). 

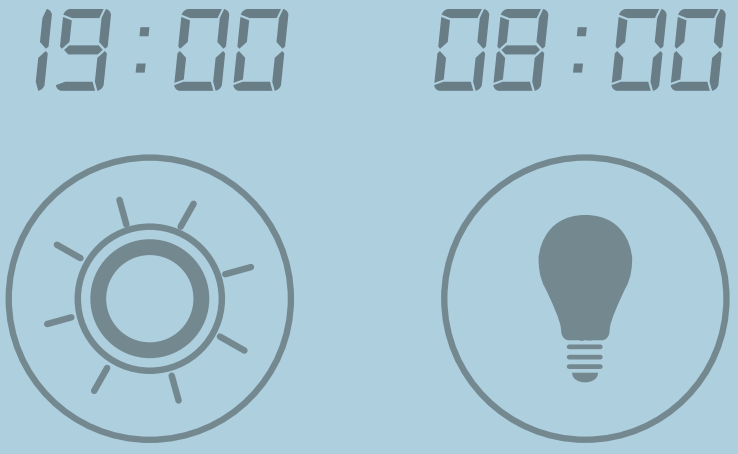

In the darkness, a sleep lab researcher is concealed behind an opening, monitoring the resident's brain activity during their sleep (Figure 7.23). This information allows the researcher to create a specific lighting schedule that simulates the dementia resident's unique circadian rhythm. An opening in the ceiling simulates a skylight that emits artificial light to stimulate the resident. Adaptable building elements such as wallpaper, furnishings and personal photographs reflect each new resident to create a familial environment upon awakening. 

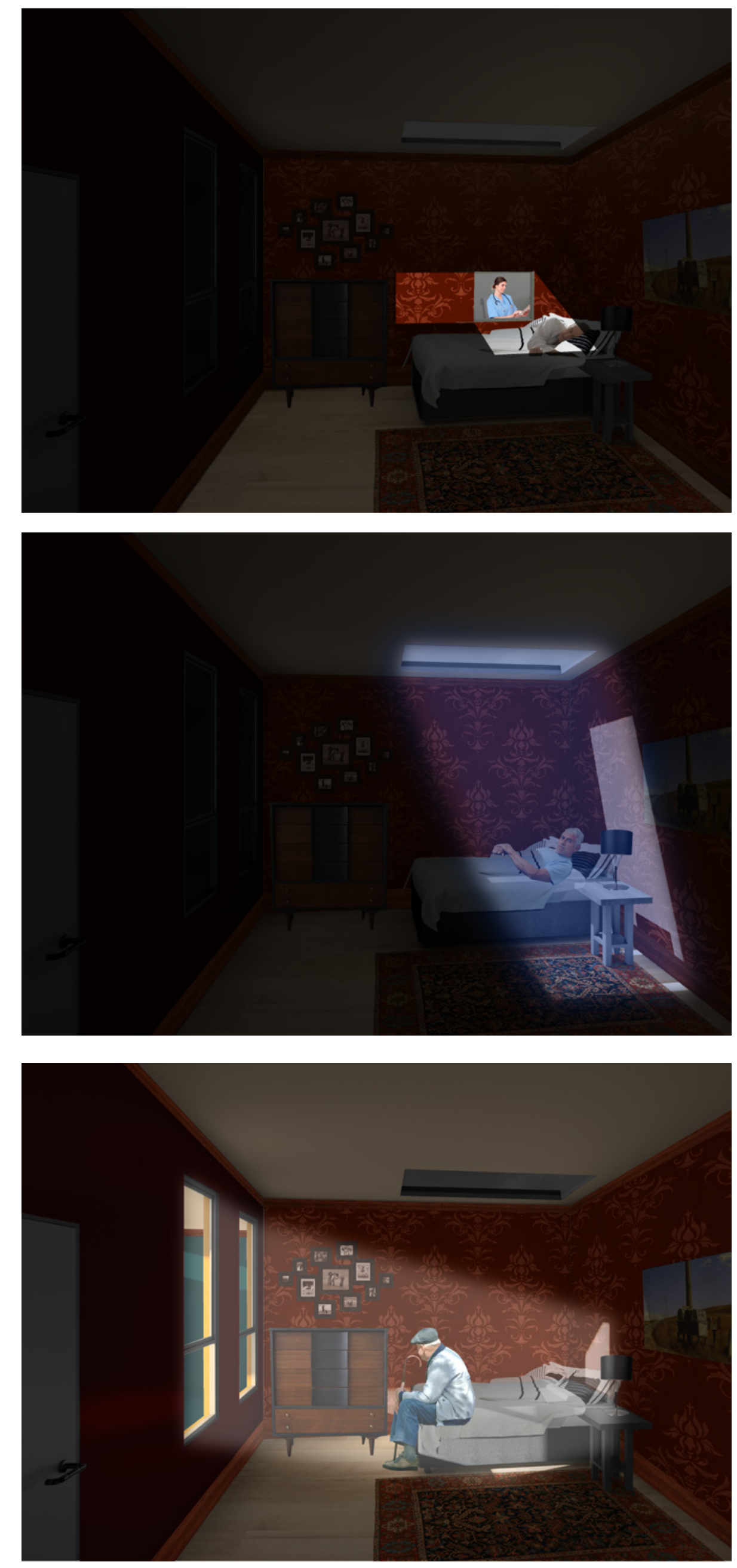

7.23 Artificial morning light stimulation 


\section{घ! :}
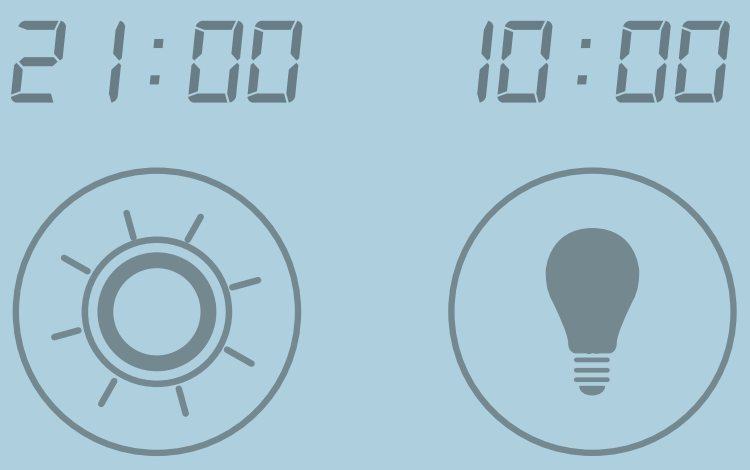

The lighting lab follows a courtyard typology, with an enclosed central green space that simulates an outdoor environment to reduce any feelings of confinement within the sleep lab. A looped circulation path alongside the courtyard reduces confusion and disorientation within the lab, with large windows providing clear lines of sight between spaces (Figure 7.24). An open planned kitchen/ living space provides opportunities for entertainment and socialization for residents and staff, aiming to reduce the sense of isolation for residents during their stay (Figure 7.25).

The artificial outdoor space contains flora and fauna that can grow in an artificial climate to provide a multisensory environment for residents. It also provides opportunities for gardening to simulate outdoor activity (Figure 7.26). The use of transparent corrugated polycarbonate sheets simulate a covered outdoor space; the material reduces visual access to the lighting lab exterior to maintain the simulated outdoor setting.
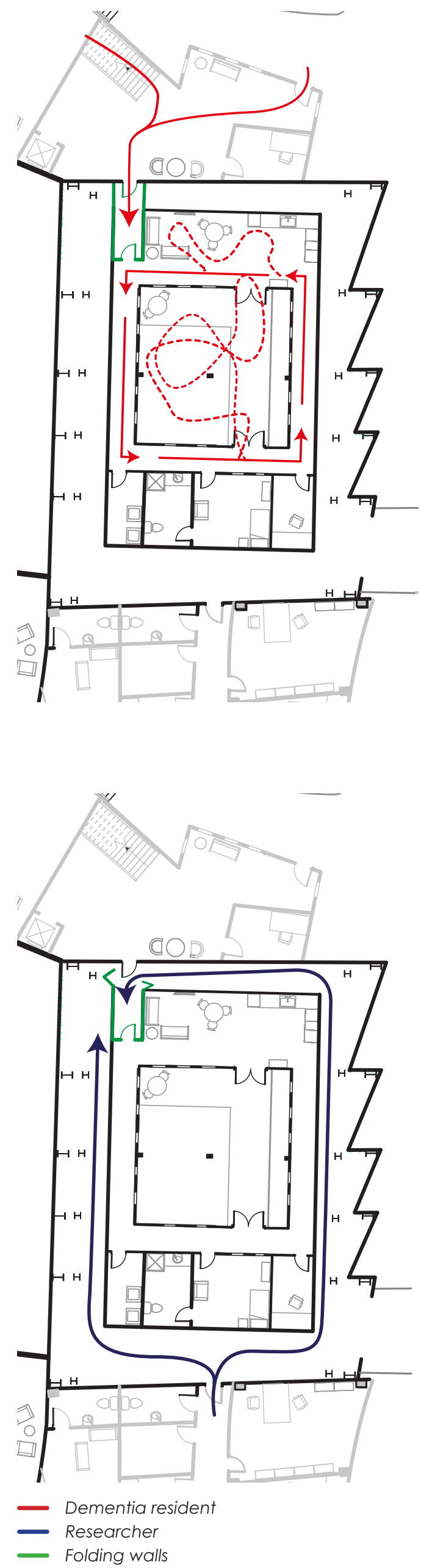

7.24 Circulation routes 


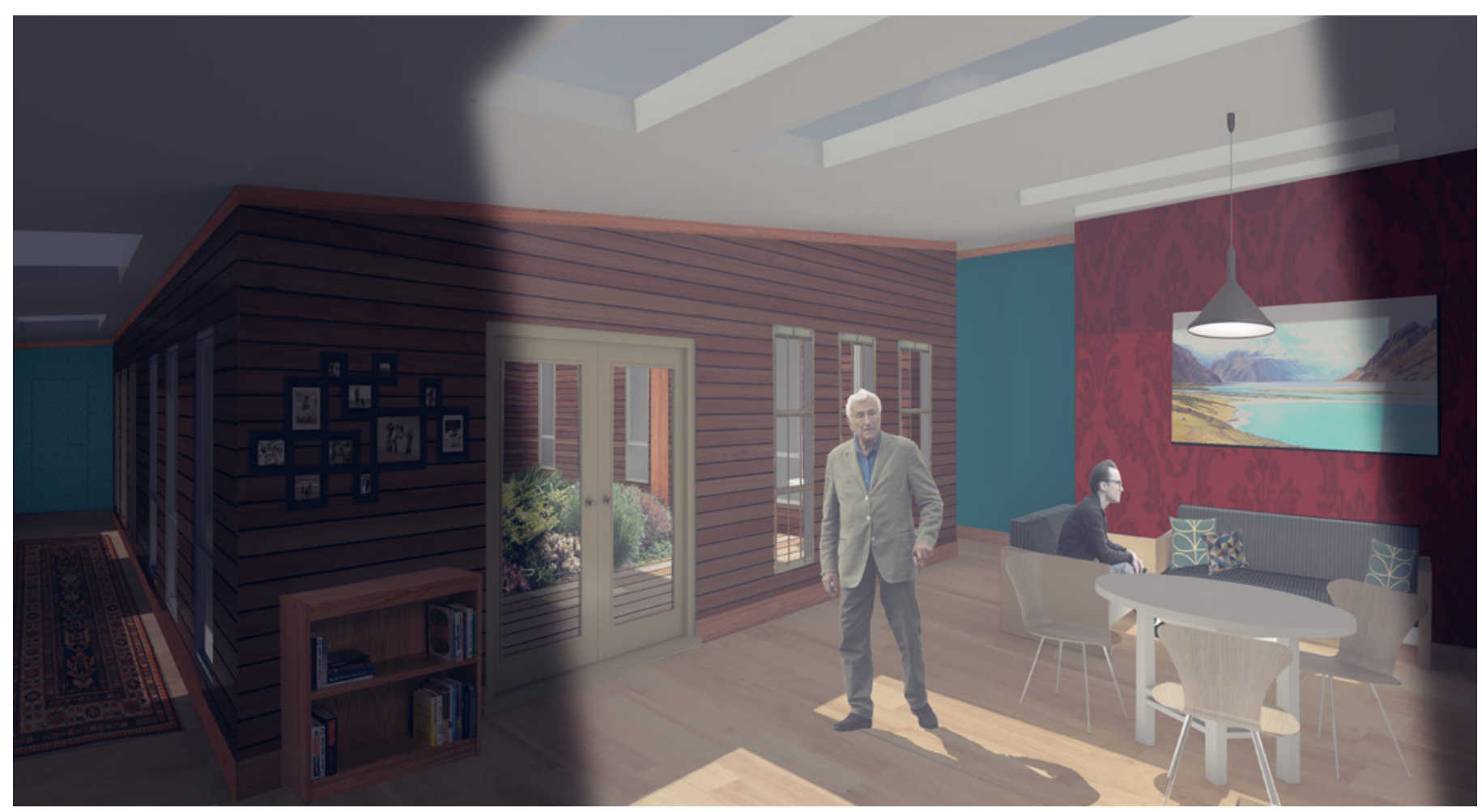

7.25 Kitchen + living room perspective

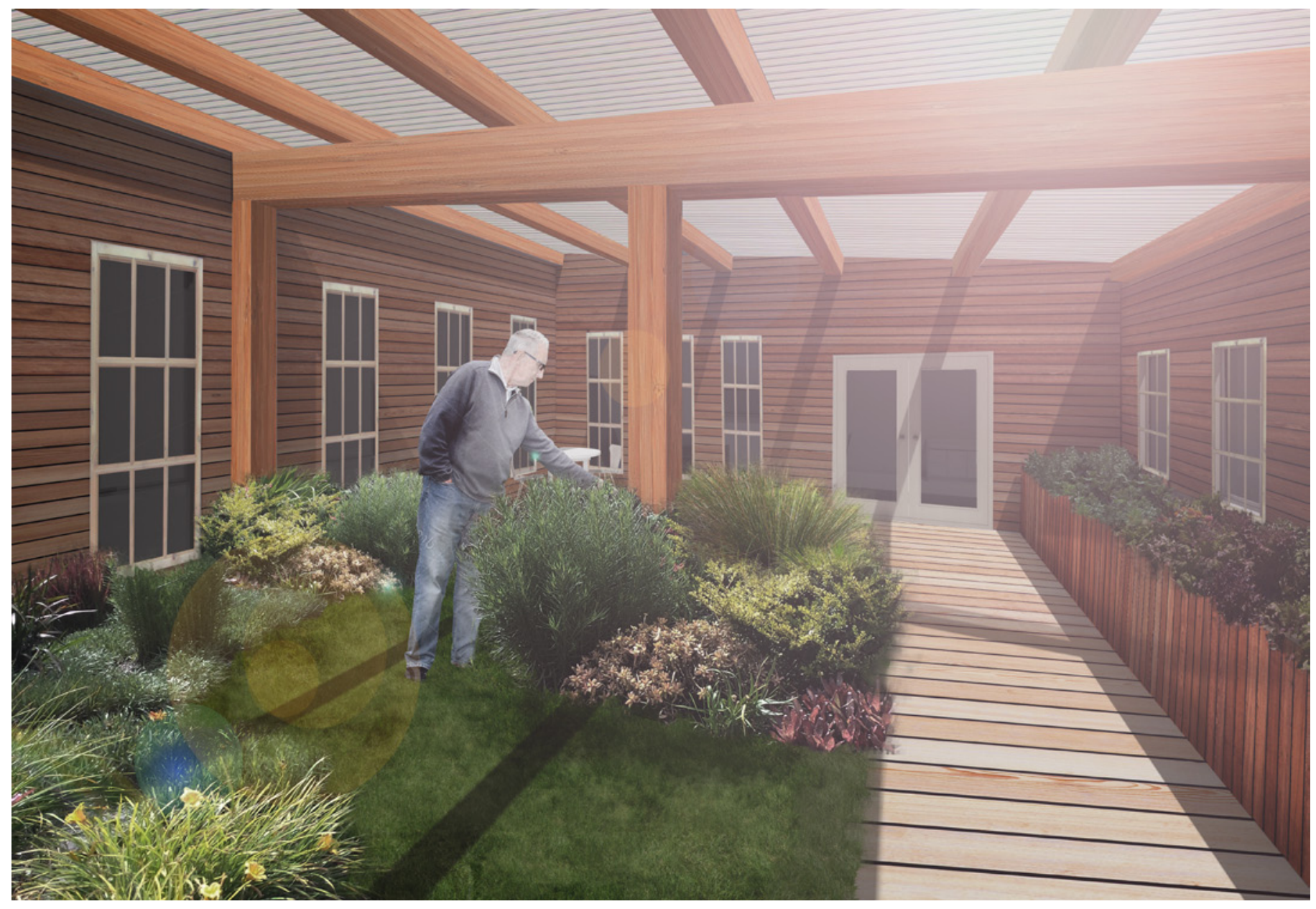

7.26 Artificial outdoor courtyard 


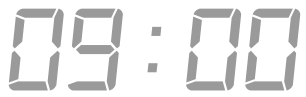

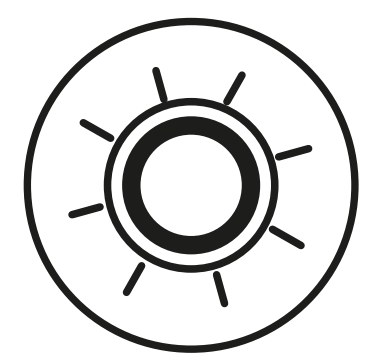

The sleep lab can test variations in colour, intensity and movement of artificial light to simulate natural light qualities and mimic the circadian rhythms of the resident (Figure 7.28). The ability for researchers to manipulate these variables allows theories to be tested and new scientific research to be explored. How does exposure to 2,000 lux of blue light (450 - $495 \mathrm{~nm}$ ) throughout the day affect a residents sleeping behaviour? (Figure 7.27) Can exposure to low frequency wavelengths (590-750nm) during the afternoon reduce the effects of sun downing?
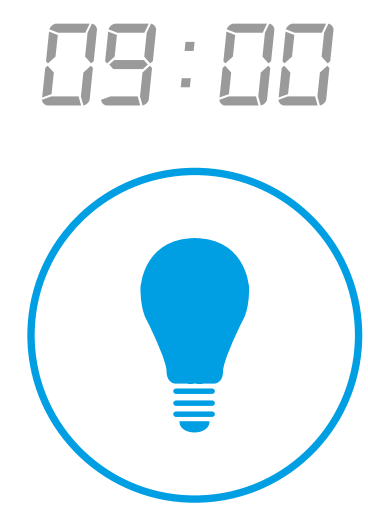

Testing these hypotheses within the lab offer opportunities to increase our understanding of the relationship between light and sleep for dementia care practice. The opportunities for the sleep lab to improve the quality of environmental research as well as the therapeutic potential of future dementia care environments helps to establish the facility as a point of interest within domestic and overseas medical research communities.

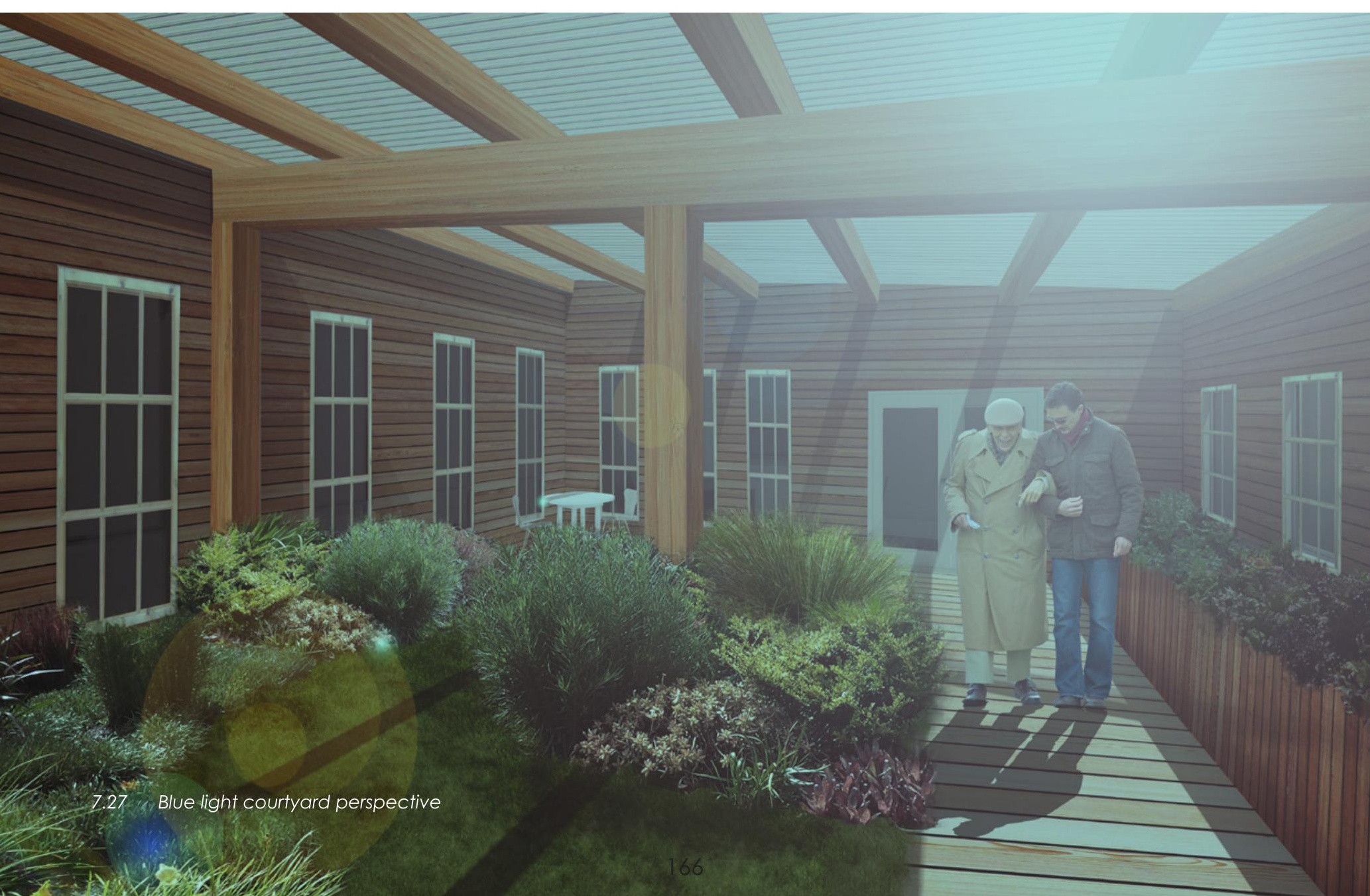


Colour
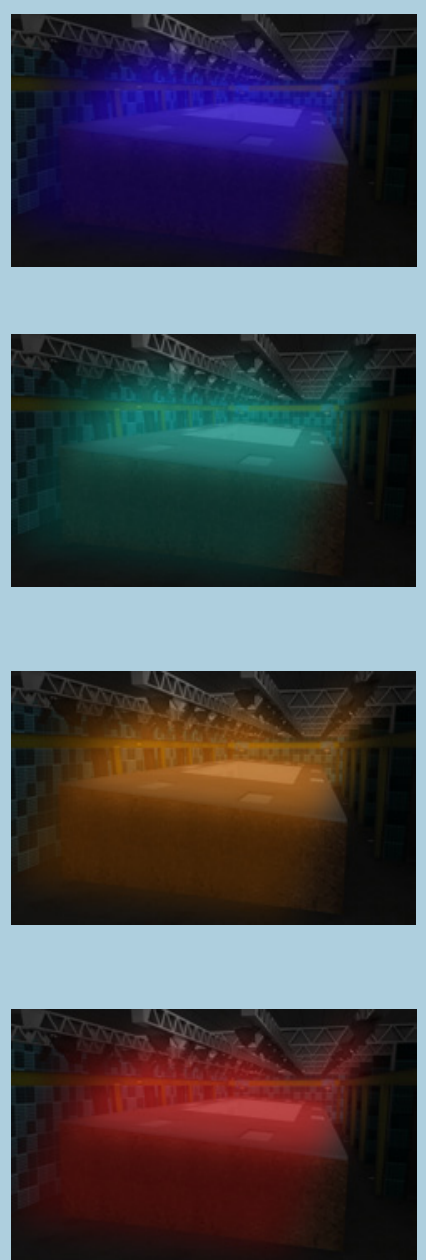

Intensity
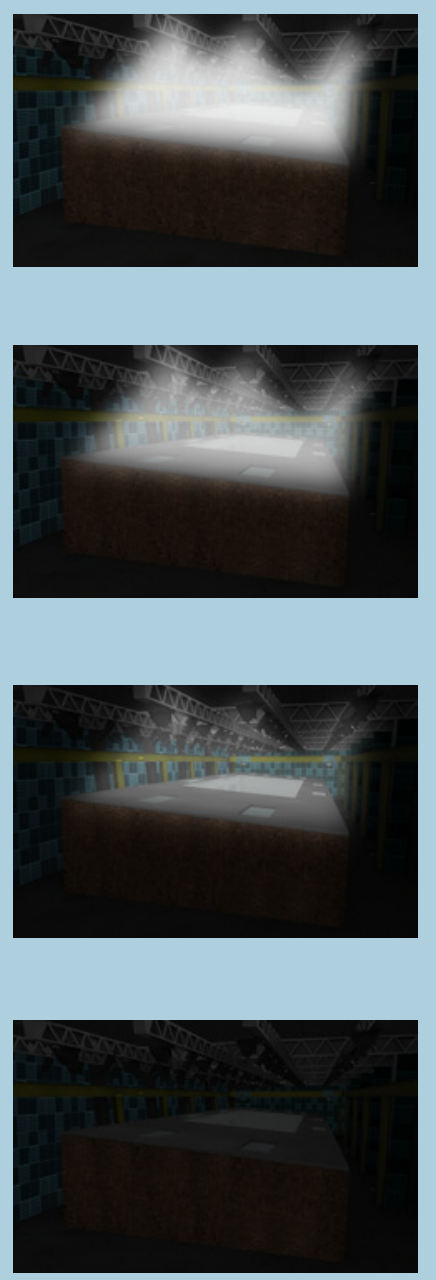

7.28
Movement
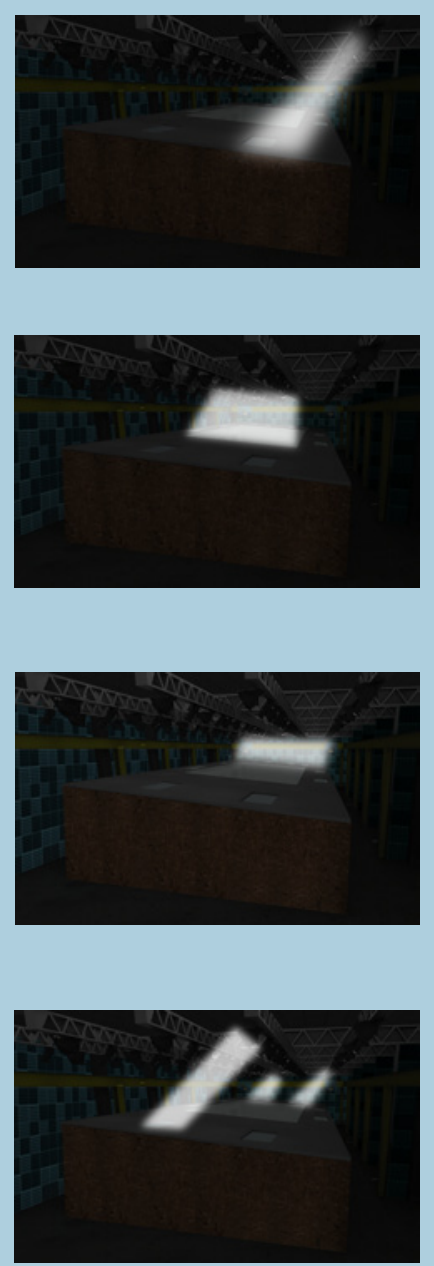


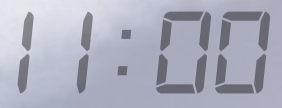
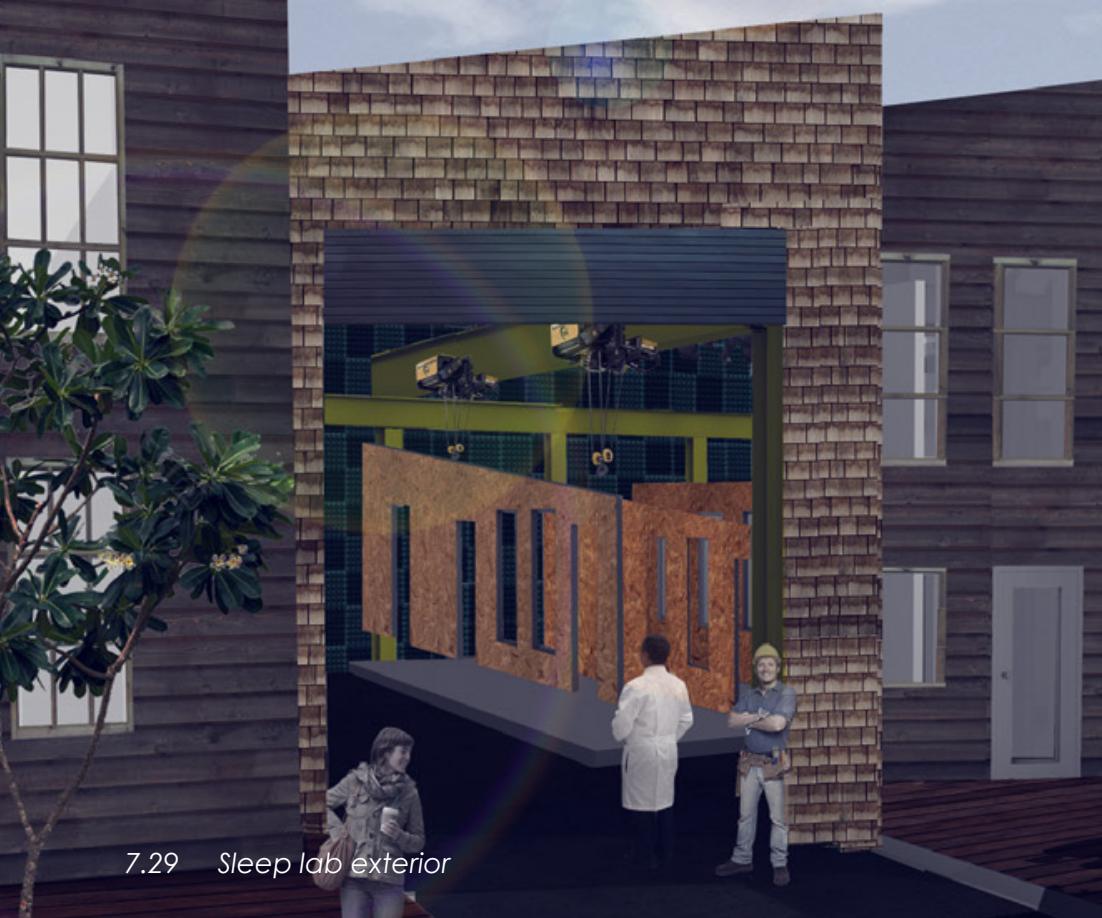

2in

in

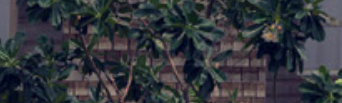

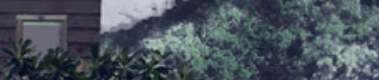

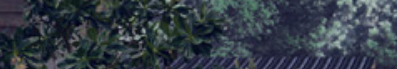
ent 30

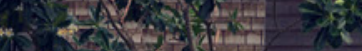
$4 x-a^{2}$

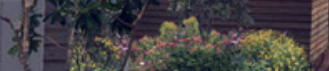

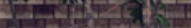

nite

10 (4)

17

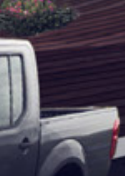



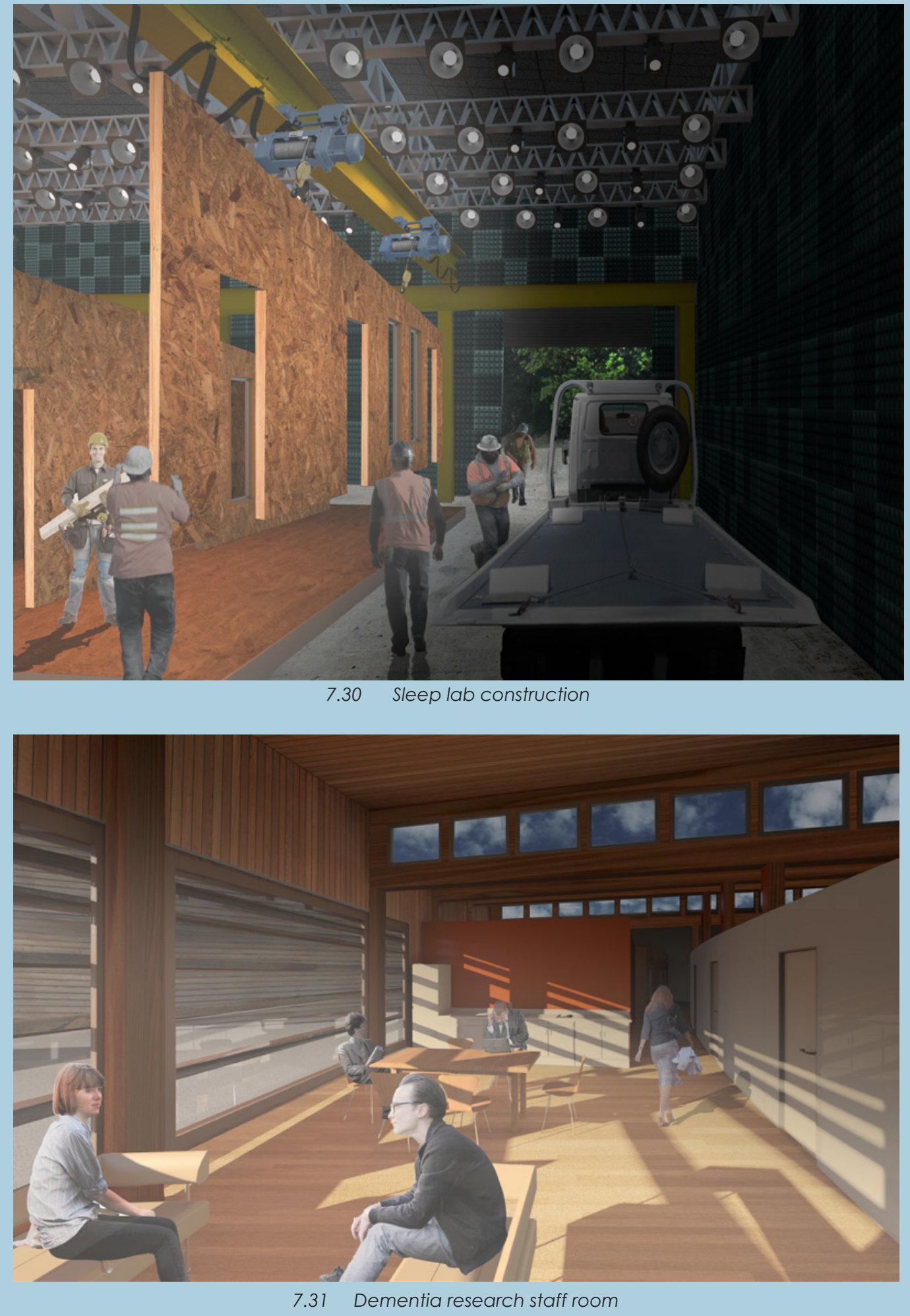

As a functioning laboratory, the integration of mechanical equipment, accessibility for vehicles through a large roller door and a spacious interior, allow for customization of the sleep lab to cater to specific groups or individuals through variation of form, spatial layout and materiality of the lab (Figure 7.29 - 7.30). As the sleep lab often requires residents to stay within these artificial environments for long periods of time, accounting for flexibility in the design of the lab allows for a person centered approach to sleep therapy and research within the lab. Adjacent research offices (Figure 7.31) allow for easy access for researchers and medical staff to monitor a resident's sleep behaviour and to provide 24-hour support for the resident to reduce feelings of loneliness during their stay. 


\section{惽:}

The exterior profile of the lab imitates a domestic aesthetic to allow the lab to blend within its surroundings and reduce the anxiety of residents who approach the lab for therapy. As evening approaches, light emitting panels transform the building and walkway with colour and vibrancy (Figure 7.32). Creating a stimulating environment for visitors and nearby residents who walk past the sleep lab in the evening. 


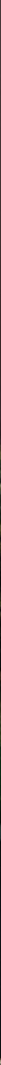




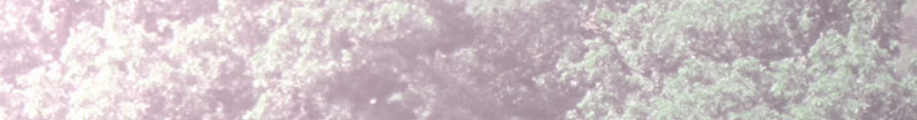

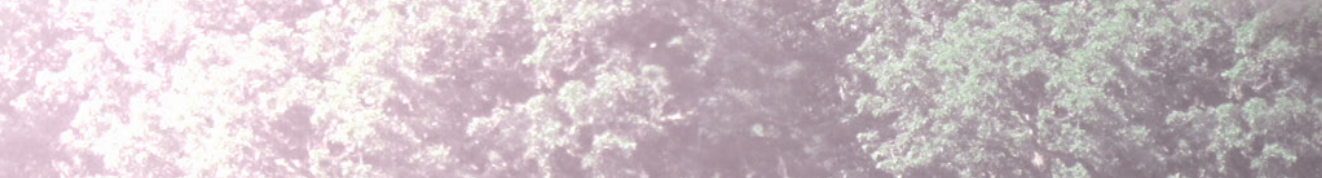

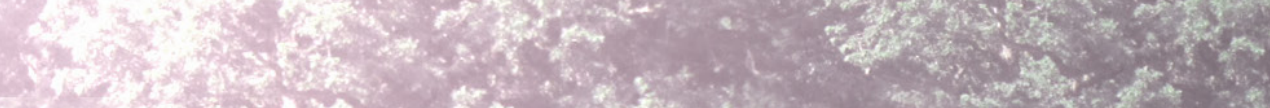

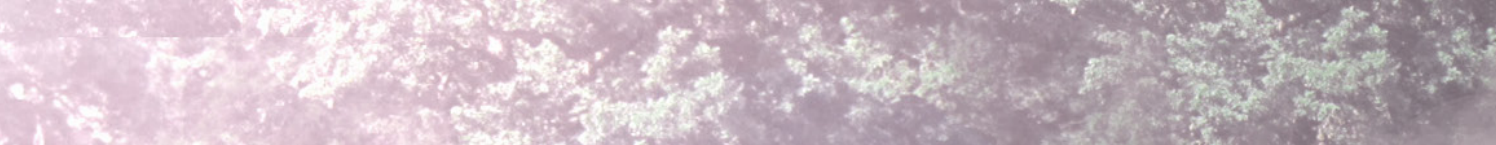
a.

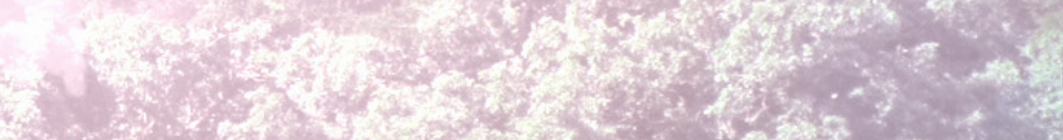

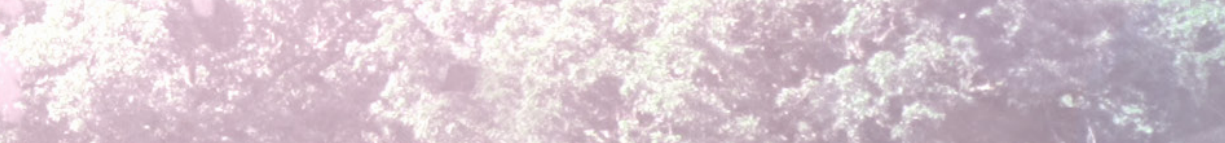

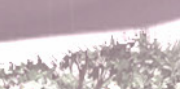

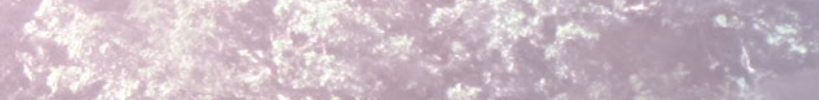

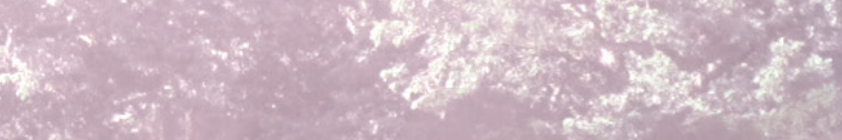

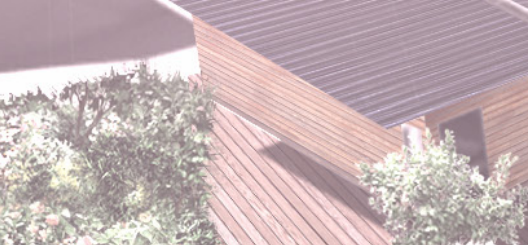
30.12

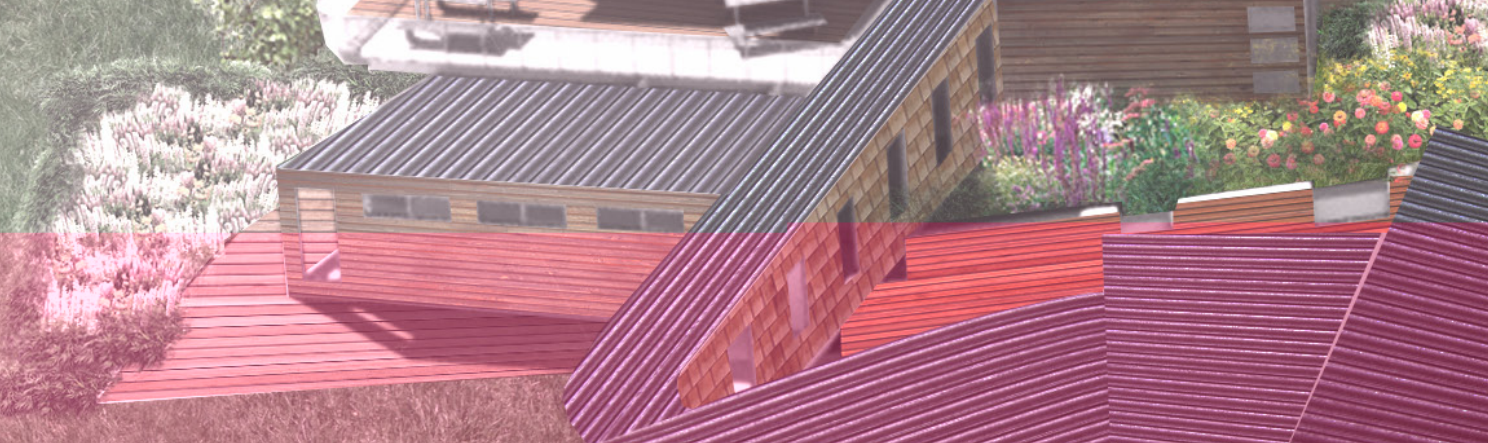

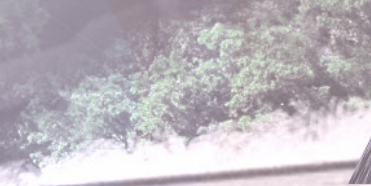

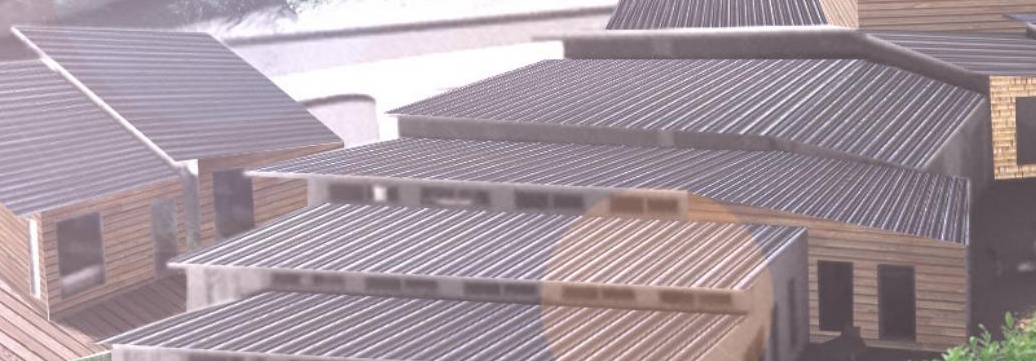
7.t.
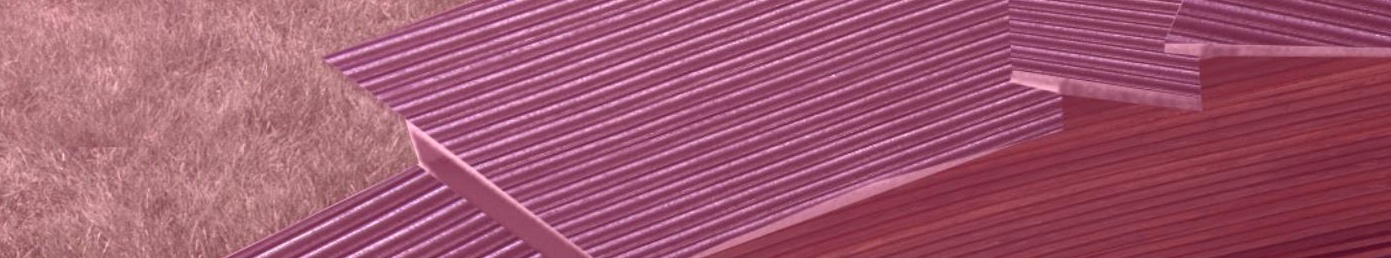

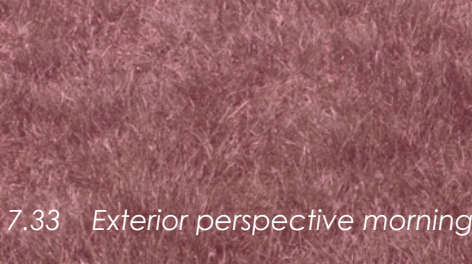

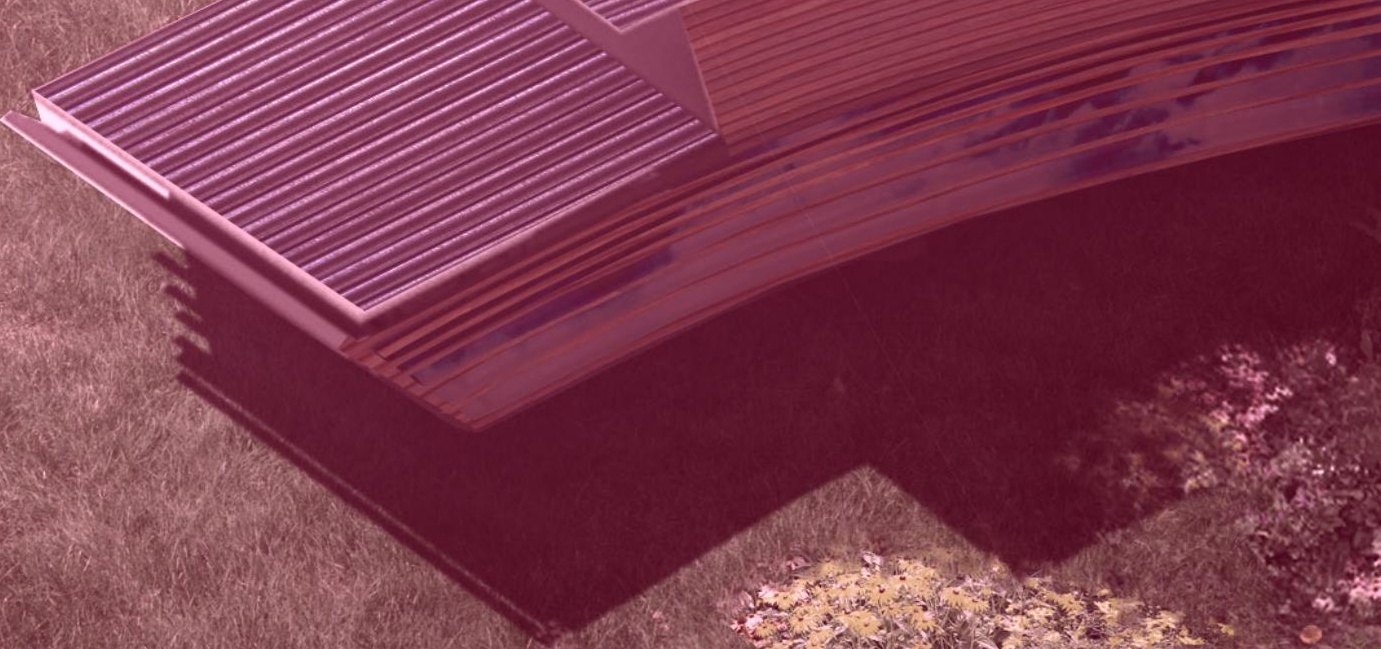

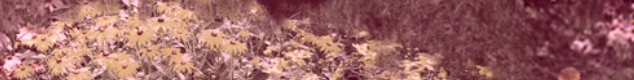

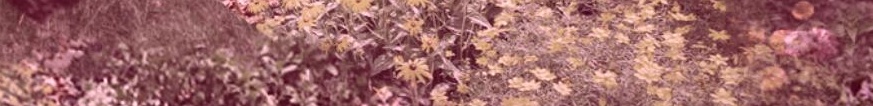

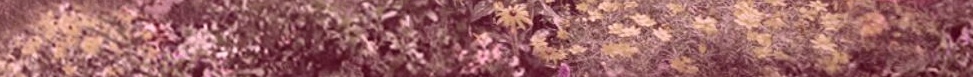

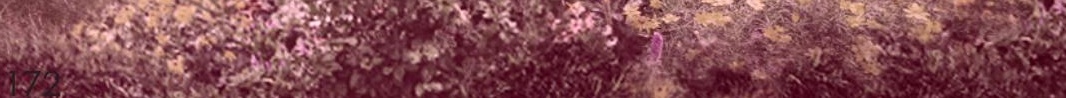





\section{8}

Conclusion + Discussion 


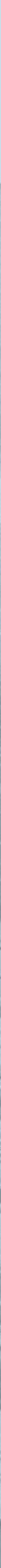


Disruptive sleeping behaviours are a key symptom of dementia that leads to early transition into full time institutional care. While respite facilities offer temporary relief for families in order to prolong home care, the use of pharmaceutical interventions coupled with unstimulating institutional environments further deteriorate sleeping symptoms upon return. To date, New Zealand's response towards dementia research maintains a focus on pharmaceutical intervention, reducing the significance of the built environment and its potential for sleep therapy. In response to the apparent negligence of the built environment in providing treatment for sleep, this thesis questions: How can the architecture of a respite facility improve sleeping behaviour and instigate meaningful environmental research within dementia care?

An interdisciplinary literature review identified light, movement and community as key environmental strategies to improve sleeping behaviour and established a design criteria to meet these desired objectives. International and local precedents were analyzed against this criteria to assess design methods to implement or avoid in achieving these desired objectives.

An "Outside-In" approach to design phase one focused on methods to attract and establish a community environment for dementia residents, developing the notion of a shared courtyard that allowed a safe environment for residents and the medical community to interact. Furthermore, the design addressed the need to communicate awareness of the buildings function and establish the facility as a point of interest on site. Although the design conveyed the notion of environmental therapy through form and aesthetic, its overarching interest to the public failed to balance the wellbeing and interests of dementia residents.
The "Inside -Out" approach to design phase two sought to balance the needs of the dementia resident by testing the relationship of light and movement at an intimate scale. The processes eventually lead to the manipulation of the exterior form from design phase one to provide a successful balance for both residents and the medical community. However, the inherent limitations of natural light to influence circadian rhythms were deemed ineffective as dementia symptoms progressed, reducing the designs effectiveness for sleep therapy.

"Simulation" was adopted in design phase three to develop a model for sleep research for later stages of dementia. The sleep lab proposed a simulated environment that used pre-existing methods of sleep treatment that would cater to the needs for those affected by dementia. The design was considered a success in creating a unique opportunity for developing environmental research for sleep therapy.

"Guiding light" represents a developed design from previous explorations in response to the research proposition and its set objectives. Through the integration of light, movement and community, Guiding Light proposes an alternative approach to respite care that sought to improve sleep behaviour and instigate meaningful environmental research towards sleep therapy for those affected by dementia. 

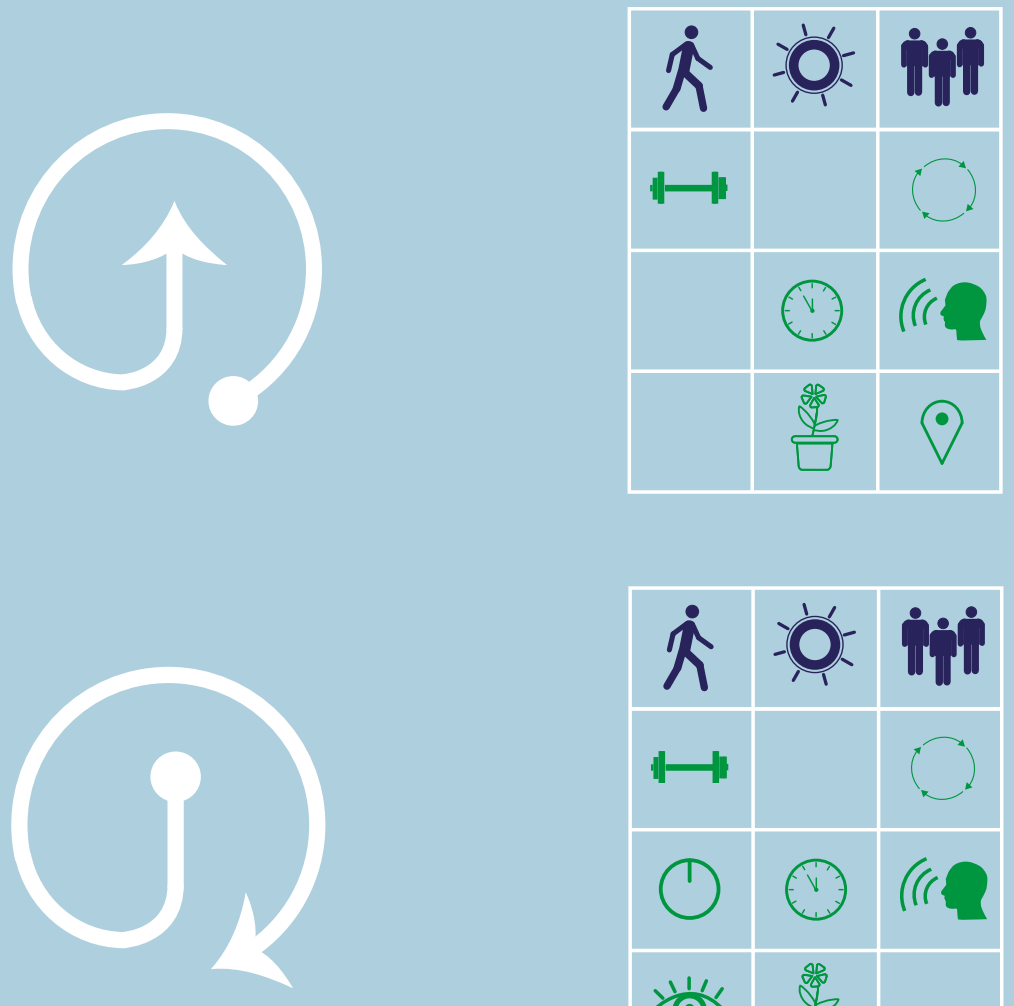

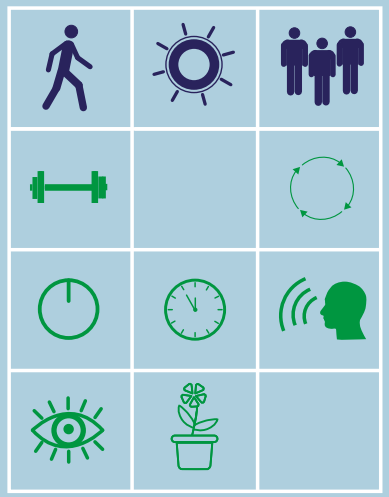
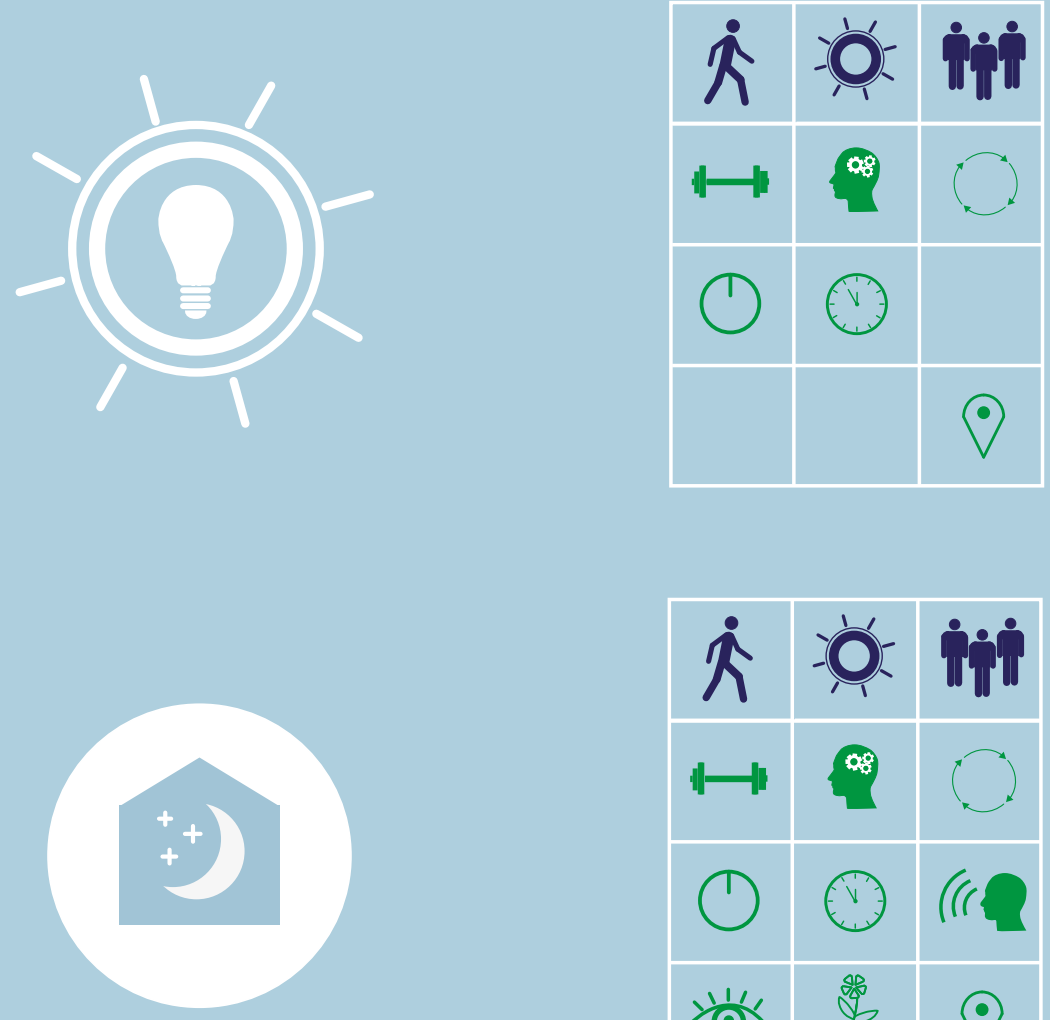

\begin{tabular}{|c|c|c|}
\hline & -'O'- & irvi \\
\hline$\leftrightarrow$ & $\oplus$ & 5 \\
\hline 0 & (1) & \\
\hline 悠" & 蛋 & \\
\hline
\end{tabular}




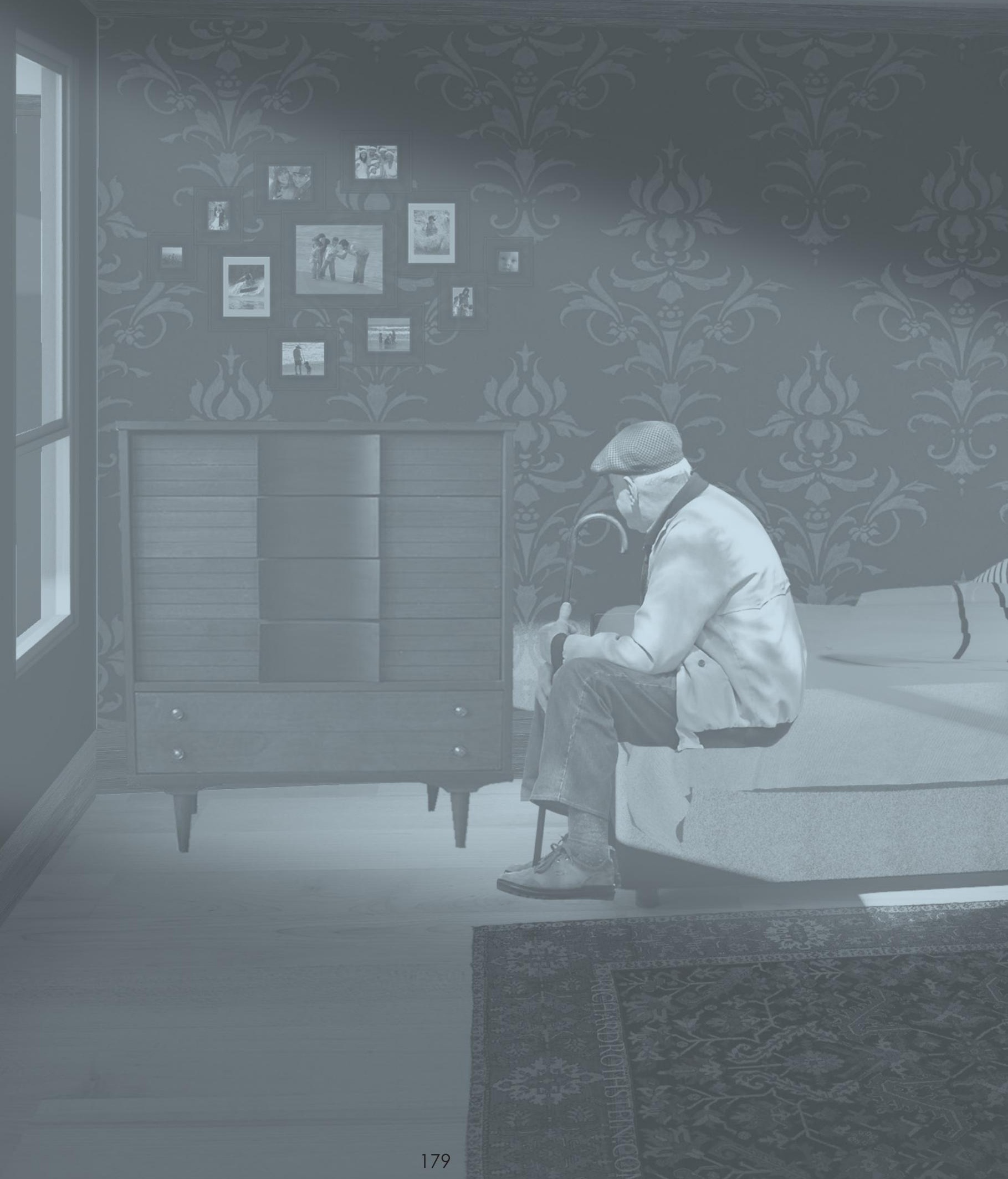



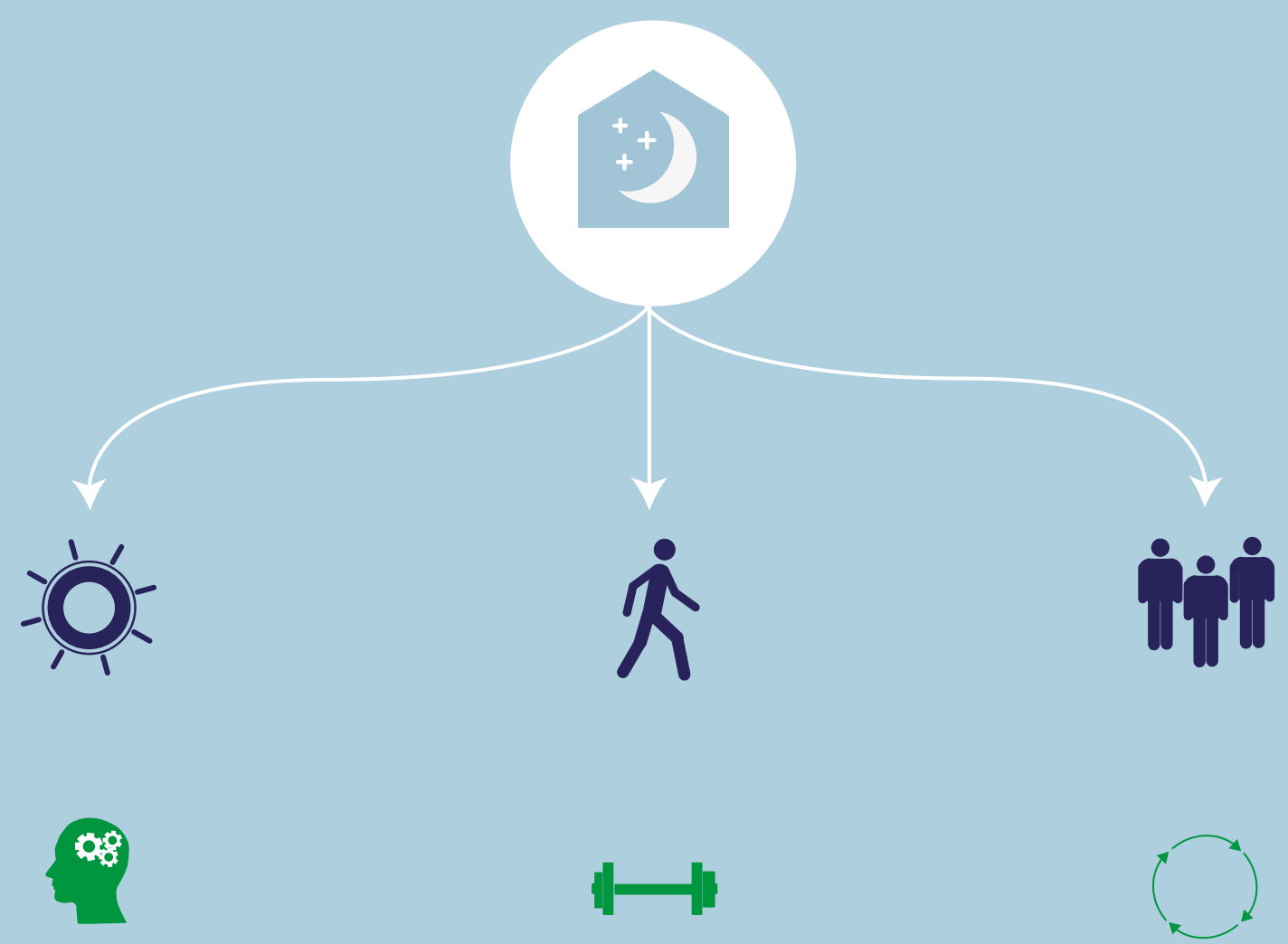

The building uses both natural and artificial lighting environments to stimulate residents during different stages of dementia

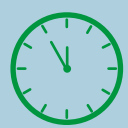

The position and orientation of building programs coincide with the daily rhythms of the sun; ensuring residents receive maximum daylight exposure available on site.

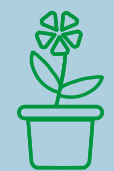

A central courtyard provides a multisensory environment that offers opportunities for outdoor activity, exposure to sunlight and engagement with nature
Dispersed programs along an enclosed infinity loop allow for exercise for residents, prompting increased mobility through daily movement patterns within the building.

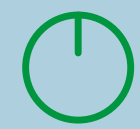

Simple circulation path with views towards central courtyard provide sense of autonomy for residents by reducing confusion and decisionmaking as they wander through the building

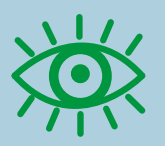

Variations in timber cladding, colour as well as interior wallpaper textures help to facilitate as environmental indicators for residents and act as visual reference points along wandering path
Integration of research, education and short term accommodation facilities provide a unique identify for respite care as a place for information and support within the community

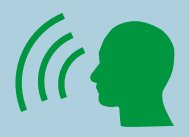

Integration of a public courtyard establishes a central hub for social activity between residents and the medical community, with predominant views towards courtyard heightening the space for an engaging and stimulating environment

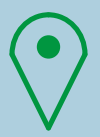

The purpose built sleep lab offers a unique opportunity for environmental research towards sleep therapy, creating a unique image for New Zealand research within an international context 
Although there is no cure for dementia, the focus of this thesis was to explore the therapeutic potential of environmental interventions towards slowing its progression. By recognizing the growing shift towards the environment as a tool for therapy, this research has developed a new design framework that targets sleep therapy within respite facilities (Figure 8.4), aiming to reduce the effect of disruptive sleeping behaviours upon family caregivers that could help prolong home care. While the research identified design methods to facilitate the objectives towards improving sleep, it was also apparent within the process the inherent limitations of environmental therapy as dementia symptoms progressed. Constrained by the progressive nature of dementia towards the design, the architectural response of the sleep lab reversed this constraint into an opportunity for architectural enquiry, where the disruptive sleeping symptoms of dementia manipulated the environment to reflect their unique circadian rhythms. This approach follows contemporary trends within the design of dementia care environments that seek to reflect rather than suppress the behavioural qualities of dementia. With dementia rates expected to rise, the demand for the design of care facilities to be more proactive in their therapeutic role alongside pharmaceutical intervention will become increasingly necessary to benefit the needs of residents and families in the future. 
9

Reference List 
Alzheimers New Zealand. "Awareness and Understanding of Dementia in New Zealand Final Report". 2014. Web. 24 July. 2015. <http://www.alzheimers.org.nz/getmedia/57831629-45cf-42628c45-2e70bl6fbfe8/Awareness_and_Understanding_of_Dementia_in_NZ_2014.pdf.aspx >

Bartle, Caroline. "Seeking Sweet Dreams: The difficulties with sleep and dementia". 25 Sep.2015. Web. 10 Nov.2015. < http://www.insitemagazine.co.nz/issues/september-2015/seeking-sweetdreams-the-difficulties-with-sleep-and-dementia/\#.VntUlcB94ch>

Baudrillard, Jean. Simulacra and Simulation. Ann Arbor: University of Michigan, 1994. Print.

Boltz, Marie. Glavin, James E. Dementia Care. An Evidence- Based Approach. 2016. Springer International Publishing. Web. 11 Nov. 2015. <https://books.google.co.nz/books? id=YwOkCgA AQBAJ\&pg $=P A 128 \& \mid p g=P A 128 \& d q=$ Dementia+Care:+An+Evidence-Based+Approach+citation \&source=bl\&ots=bFddcvyusG\&sig=vL95-NJJhitP8dAzTWyE5B5PfCU\&hl=en\&sa=X\&ved=0ahUKEW jfx63MrPPJAhUGOKYKHRM_DDQQ6AEIPZAF\#v=onepage\&q=Dementia\%20Care\%3A\%20An\%20 Evidence-Based\%20Approach\%20citation\&f=false>

Boubekri, Mohamed. Daylighting, Architecture, and Health : Building Design Strategies. 1st ed. Amsterdam : Elsevier Architectural Press, 2008. Print.

Calkins, Margaret P. "Evidence-based long term care design."NeuroRehabilitation 25.3 (2009): 145-154.Print.

Chalfont, Garuth Eliot. "Connection to nature at the building edge: towards a therapeutic architecture for dementia care environments."Diss., The University of Sheffield (2006). Web.

< http://etheses.whiterose.ac.uk/1241/2/Chalfont,_Garuth_Eliot.pdf>

Cipriani, Gabriele, et al. "Wandering and dementia." Psychogeriatrics 14.2 (2014): 135-142. Print.

Desiree A. Lie. "Bright Light Therapy in Dementia". 21 July. 2015. Web. 12 Oct. 2015. <http://www. medscape.com/viewarticle/848045_2>

Feddersen, Eckhard, and Insa Lüdtke. Lost in Space: Architecture and Dementia.Basel: Birkhauser, 2014. Print.

Gibson, Rosemary. "Sleep and Dementia". Massey University Sleep/Wake Research Centre. 4 May 2015. Microsoft PowerPoint file.

Gooley, Joshua J. "Treatment of circadian rhythm sleep disorders with light." Ann Acad Med Singapore 37.8 (2008): 669-676. Print.

Klaffke, S and J. Staedt. "Sundowing and circadian rhythm disorders in dementia." Acta neurologica belgica 106.4 (2006): 168-175. Print.

Knowles, Ralph L. Ritual House : Drawing on Nature's Rhythms for Architecture and Urban Design. 1st ed. Washington, D.C: Island Press, 2006. Print.

Lee, David, Kevin Morgan, and James Lindesay. "Effect of institutional respite care on the sleep of people with dementia and their primary caregivers." Journal of the American Geriatrics Society 55.2 (2007): 252-258. Print.

Mathewson, Nicole. "Dementia plan urged as Kiwis age". 2013. Web. 20 March .2015.

<http://www.stuff.co.nz/national/health/8397671/Dementia-plan-urged-as-Kiwis-age> 
McCurry, Susan M., et al. "Successful behavioural treatment for reported sleep problems in elderly caregivers of dementia patients: a controlled study." The Journals of Gerontology Series B: Psychological Sciences and Social Sciences 53.2 (1998): P122-P129.Print.

Moneo, Rafael. "On typology." Oppositions 13.1 (1978): 22-45. Print.

O'Brian, Darragh. Ed. "Aged Care" Evidence Based Design (2014). Print.

Radio New Zealand. "Dementia Cases Expected to Triple". 26 Aug. 2015. Web. 05 Nov. 2015.

< http://www.radionz.co.nz/news/national/282453/dementia-cases-expected-to-triple>

Reimer, Marlene A., et al. "Special care facility compared with traditional environments for dementia care: a longitudinal study of quality of life." Journal of the American Geriatrics Society 52.7 (2004): 1085-1092. Print.

Richards, Kathy Culpepper,. E† al. "Effect of Individualized Social Activity on Sleep in Nursing Home Residents with Dementia." Journal of the American Geriatrics Society: 1510 -517.Print.

The New Zealand Herald. "NZ dementia rates set to double". 2 Jan. 2012. Web. 04 Apr. 2015. <http://www.nzherald.co.nz/world/news/article.cfm?.c_id=2\&objectid=10775013>

The University of Auckland. "First dementia research clinic for New Zealand". 21 July.2014. Web. 04 Apr. 2015. <https://www.fmhs.auckland.ac.nz/en/faculty/about/news-and-events/ news/2014/07/21/First-dementia-research-clinic-for-New-Zealand.html>.

Tschumi, Bernard. Architecture Concepts : Red Is Not a Color. New York : Enfield: Rizzoli ; Group UK, 2012. Print.

Werner, Perla, et al. "Family stigma and caregiver burden in Alzheimer's disease." The Gerontologist 52.1 (2012): 89-97. Print.

Whear, Rebecca, et al. "What is the impact of using outdoor spaces such as gardens on the physical and mental well-being of those with dementia? A systematic review of quantitative and qualitative evidence." Journal of the American Medical Directors Association 15.10 (2014): 697705. Print.

Williams, Huw "Garden therapy could bear fruit for dementia care". 20 Sep.201 1.Web.15.Apr.2015. <http://www.bbc.com/news/uk-scotland-tayside-central-14979146> 
10

List Of Figures 
All images owned by the author unless otherwise stated

\subsection{Dementia Home Care}

Winter, Nita. The Price of Caregiving. 2008. Labour Projects for Working Families. Web. 15 Dec. 2015. <http://www.workingfamilies.org/publications/newsletter/summer08.html>

\subsection{Lighting system to provide high circadian stimulation}

Lighting Research Center at Rensselaer Polytechnic Institute. Lighting system to provide high circadian stimulation. 2015. Medscape. Web. 12 Oct. 2015. <http://www.medscape.com/ viewarticle/848045_2>

\subsection{Circulation routes integrated with outdoor spaces}

Johnson, Clare. The Benefits of Outdoor Spaces for the Elderly. 2014. My Chicago Botanic Garden. Web. 13 Dec 2015. < http://my.chicagobotanic.org/education/therapy/the-benefits-of-outdoorspaces-for-the-elderly/>

2.8 Social activities allowing for cognitive stimulation

Fielding, Paula. Social Engagement 'May Lower Risk Of Cognitive Decline'.2015. Sunrise Senior Living Blog. Web. 15 Dec 2015. <http://www.sunrise-care.co.uk/blog/april-2015/social-engagementmay-lower-risk-of-cognitive-decline.aspx>

\subsection{Alzheimer's Respite Centre Exterior}

Niall Mclaughlin Architects. View of the quiet room over the brick wall. 2012. Alzheimer's Respite Centre, Dublin. Web. 10 Dec. 2015. < http://www.niallmclaughlin.com/projects/alzheimers-respitecentre-dublin/>

\subsection{Outdoor Social Spaces}

Sars, Madeleine. Photograph. 2012. Dementia Village 'De Hogeweyk' in Weesp. Web. 11 Dec. 2015. < http://www.detail-online.com/article/dementia-village-de-hogeweyk-in-weesp-16433/>

\subsection{Supermarket}

KopArt. Patients can go shopping in the supermarket themselves. 2012. Dementia Village 'De Hogeweyk' in Weesp. Web. 11 Dec. 2015. < http://www.detail-online.com/article/dementiavillage-de-hogeweyk-in-weesp-16433/>

\subsection{Therapeutic Garden}

Vivium. De Hogeweyk. Web. 11 Dec. 2015. < http://www.vivium.nl/hogeweyk>

\subsection{Courtyard Space}

Sars, Madeleine. Photograph. 2012. Dementia Village 'De Hogeweyk' in Weesp. Web. 11 Dec. 2015. < http://www.detail-online.com/article/dementia-village-de-hogeweyk-in-weesp-16433/>

\subsection{Building Entrance}

CF Moller. Building Entrance Render. 2013. New Frogner Nursing Home. Web. 11 Dec. 2015.

< http://www.cfmoller.com/p/New-Frogner-Nursing-Home-i31 19.html> 


\subsection{Bedroom}

CF Moller. 2013. New Frogner Nursing Home. Web. 11 Dec. 2015. < http://www.cfmoller.com/p/ New-Frogner-Nursing-Home-i31 19.html>

\subsection{Green Atrium One}

CF Moller. 2013. New Frogner Nursing Home. Web. 11 Dec. 2015. < http://www.cfmoller.com/p/ New-Frogner-Nursing-Home-i3119.html>

\subsection{Green Atrium Two}

CF Moller. 2013. New Frogner Nursing Home. Web. 11 Dec. 2015. < http://www.cfmoller.com/p/ New-Frogner-Nursing-Home-i31 19.html>

\subsection{Box Lantern External Perspective}

Niall Mclaughlin Architects. View of the quiet room over the brick wall. 2012. Alzheimer's Respite Centre, Dublin. Web. 10 Dec. 2015. < http://www.niallmclaughlin.com/projects/alzheimersrespite-centre-dublin/>

\subsection{Seating Area}

Niall Mclaughlin Architects. Bench at the end of the bedroom wing corridor. 2012. Alzheimer's Respite Centre, Dublin. Web. 10 Dec. 2015. < http://www.niallmclaughlin.com/projects/ alzheimers-respite-centre-dublin/>

\subsection{Corridor Space}

Niall Mclaughlin Architects. The bedroom wing corridor. 2012. Alzheimer's Respite Centre, Dublin. Web. 10 Dec. 2015. < http://www.niallmclaughlin.com/projects/alzheimers-respite-centredublin/>

\subsection{Living Room}

Niall Mclaughlin Architects. The quiet room with views to the courtyard. 2012. Alzheimer's Respite Centre, Dublin. Web. 10 Dec. 2015. < http://www.niallmclaughlin.com/projects/alzheimersrespite-centre-dublin/>

\subsection{Building Entrance}

Maycroft Construction. 2012. Te Hopai Trust Rest Home. Web. 10 Dec. 2015. <http://www. maycroft.co.nz/projects.php? projectid=20>

\subsection{Courtyard}

Maycroft Construction.2012. Te Hopai Trust Rest Home. Web. 10 Dec. 2015. <http://www. maycroft.co.nz/projects. php? projectid=20>

\subsection{Corridor Space}

Maycroft Construction.2012. Te Hopai Trust Rest Home. Web. 10 Dec. 2015. <http://www. maycroft.co.nz/projects. php? projectid=20> 


\subsection{Glass Pavilion}

Sprott House. The glass pavilion, an integral part of Duncan Lodge. 2012. Duncan House Opening Photographs. Web. 10 Dec. 2015.<http://www.sprott-house.co.nz/rest-home-wellington/ dementia-secure-unit-wellington-opening-photos.php>

\subsection{Courtyard Perspective One}

Eldernet. 2015. Dementia Care (Secure Environment): Sprott House - Duncan Lodge. Web. 10 Dec. 2015. <https://www.eldernet.co.nz/Facilities/Dementia_Care_Secure_environment/Sprott_House/ Service/DisplayService/FaStID/1807>

\subsection{Courtyard Perceptive Two}

Cooke, Lisa. Enabling Independence in a Stage 3 Dementia Unit: Changing the Model of Care, the 'Spark of Life' Approach. Nov 2014. Web. 10 Dec. 2015. <https://www.pharmac.govt.nz/ assets/ss-dementia-5-enabling-independence-in-a-stage-3-dementia-unit-lisa-cooke.pdf>

\subsubsection{John Curtin School of Medical Research Façade Detail}

Lyons. John Curtin School of Medical Research Stage 1, Australian National University. Web. 14 July. 2015. < http://www.lyonsarch.com.au/anu-john-curtin-school-of-medical-research-stage/? b=1>.

\subsubsection{La Trobe University Institute For Molecular Science Façade}

Lyons. La Trobe University, Institute for Molecular Science. Web. 14 July. 2015. < http://www. lyonsarch.com.au/la-trobe-university-institute-for-molecular-science/? $b=1>$

\subsubsection{University of Tasmania School of Medicine Façade}

Lyons. UTAS School of Medicine, Stage 2. Web. 14 July.2015. < http://www.lyonsarch.com.au/utasschool-of-medicine-stage- $2 /$ ? $b=1>$ 
